Yuri Tschinkel (Ed.)

Mathematisches Institut

Georg-August-Universität Göttingen Seminars Winter Term 2004/2005

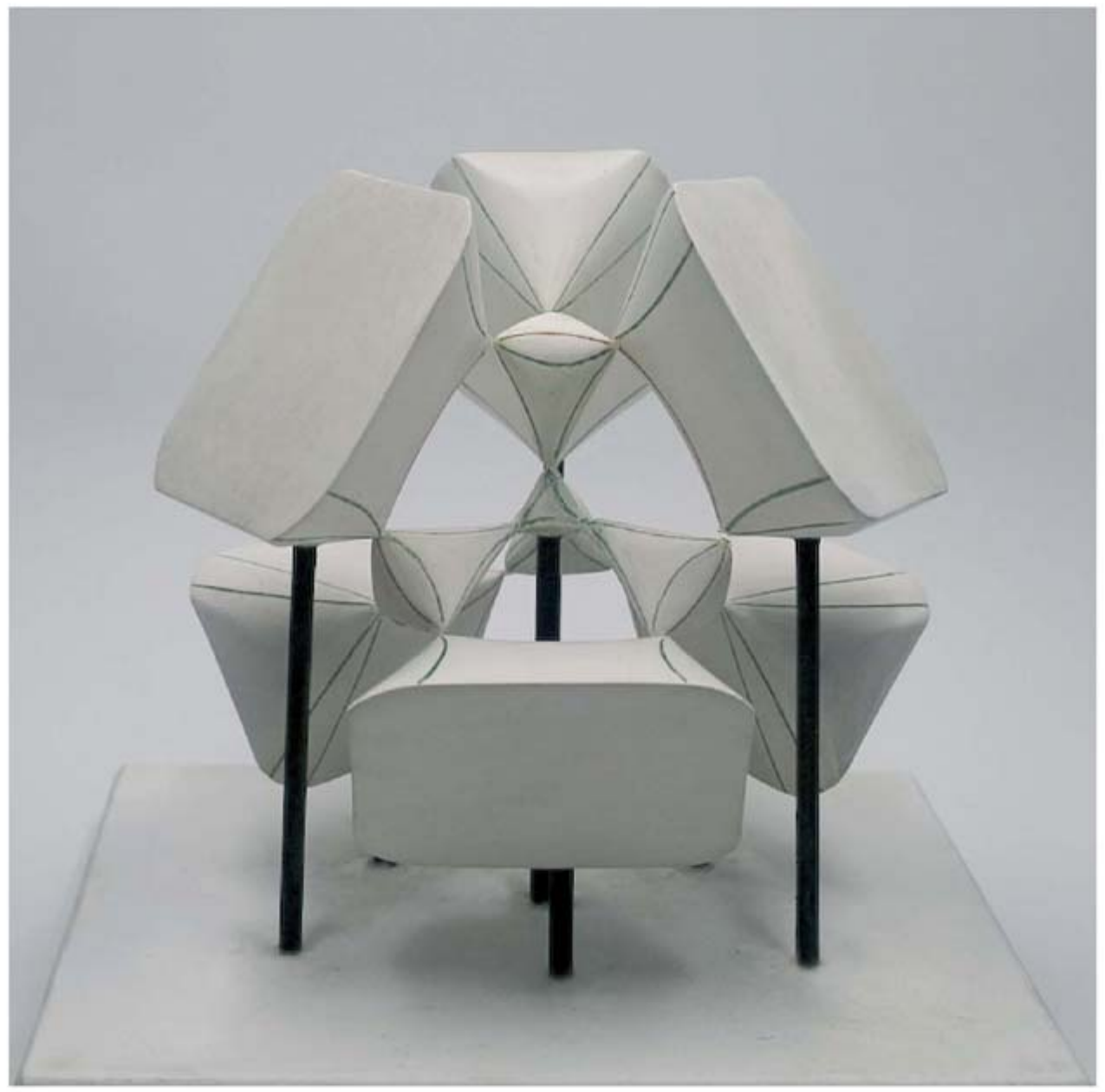

G Universitätsdrucke Göttingen 

Yuri Tschinkel (Hg.)

Mathematisches Institut Georg-August-Universität Göttingen

Seminars Winter Term 2004/2005 
erschienen in der Reihe „Mathematisches Institut. Seminare“

der Universitätsdrucke des Universitätsverlages Göttingen 2005 
Yuri Tschinkel (Hg.)

Mathematisches Institut

Georg-August-Universität

Göttingen

Seminars Winter Term

2004/2005

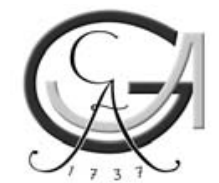

Universitätsverlag Göttingen 2005 
Bibliografische Information der Deutschen Bibliothek

Die Deutsche Bibliothek verzeichnet diese Publikation in der

Deutschen Nationalbibliographie; detaillierte bibliografische Daten sind im Internet über $<$ http//dnb.ddb.de $>$ abrufbar.

Address of the Editor / Anschrift des Herausgebers

Yuri Tschinkel

Mathematisches Institut

der Georg-August-Universität Göttingen

Bunsenstraße 3-5

37073 Göttingen

e-mail: yuri@uni-math.gwdg.de

URL http://www.uni-math.gwdg.de/tschinkel

(C) All Rights for the printed version reserved, Universitätsverlag Göttingen 2005

Cover Image by Phillip Hagedorn. Mathematisches Institut Göttingen

Cover Design Margo Bargheer

ISBN 3-938616-17-2 


\section{CONTENTS}

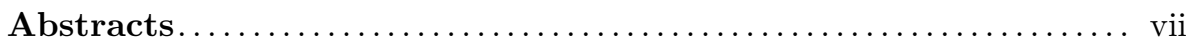

Introduction $\ldots \ldots \ldots \ldots \ldots \ldots \ldots \ldots \ldots \ldots \ldots \ldots \ldots \ldots \ldots \ldots \ldots \ldots \ldots \ldots$

J. Heinloth - Notes on differentiable Stacks ..................... 1

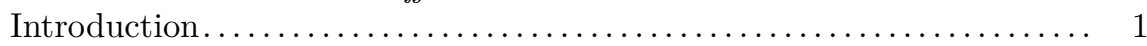

1. Motivation and the first definition of stacks................ 2

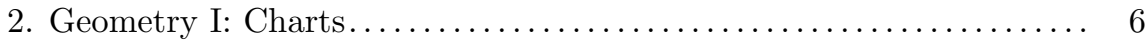

3. Topological stacks as topological groupoids................. 12

4. Geometry II: Sheaves, cohomology, tangent spaces, dimension, normal

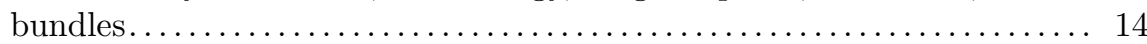

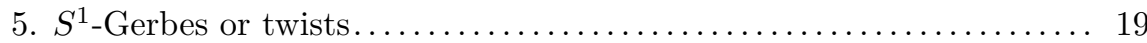

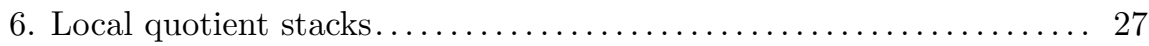

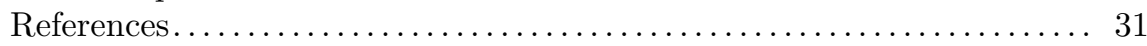

U. BunkE \& I. SCHRÖDER — Twisted K-theory and TQFT ........ 33

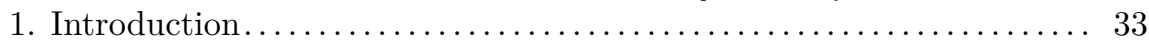

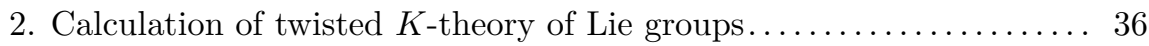

3. Moduli spaces and trivializations of twists ................ 67

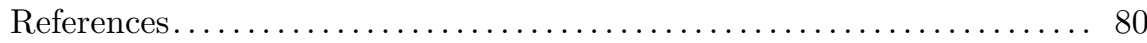

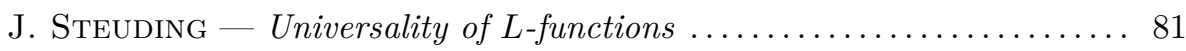

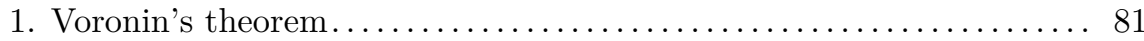

2. Zeros and the Riemann hypothesis.................... 84

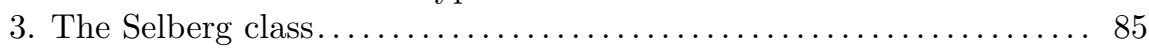

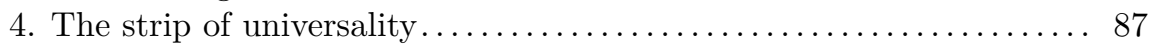

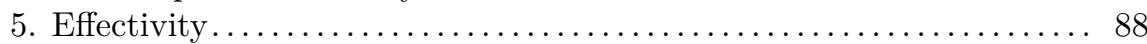

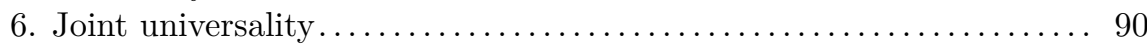

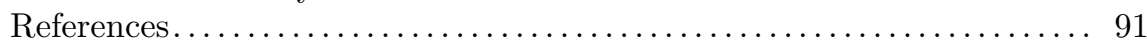


D. VOGEL - Masseyprodukte in der Galoiskohomologie von Zahlkörpern 93

1. Masseyprodukte und Relationen in pro- $p$-Gruppen............. 93

2. 2-Erweiterungen mit beschränkter Verzweigung.............. 96

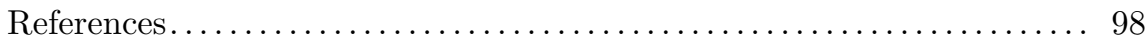

M.V. BONDARKO - Finite flat commutative group schemes over complete discrete valuation fields: classification, structural results; application to reduction of Abelian varieties ............................. 99

1. The category of finite flat commutative group schemes; the generic

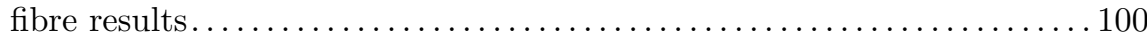

2. Formal groups; Cartier modules........................ 100

3. Cartier-Oort modules of local group schemes.................. 102

4. Finite criteria for reduction of Abelian varieties.................. 104

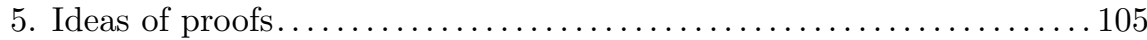

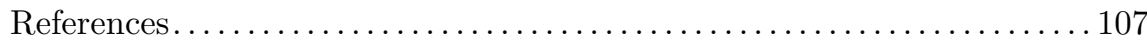

A. Szenes — Toric reduction and tropical geometry ................. 109

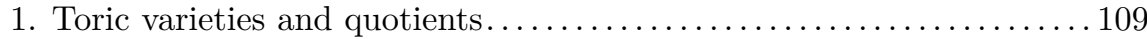

2. Intersection numbers of toric varieties..................... 112

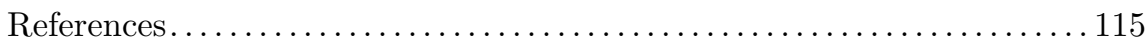

R. MEYER - A spectral interpretation for the zeros of the Riemann zeta

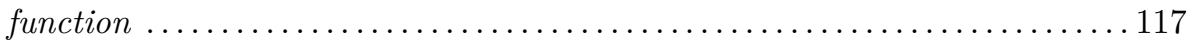

1. Introduction......................................... 117

2. The ingredients: some function spaces and operators........... 119

3. The Zeta operator and the Poisson Summation Formula........... 122

4. The spectral interpretation............................... 125

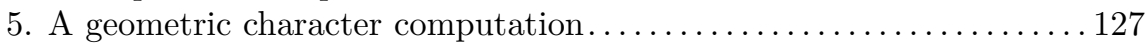

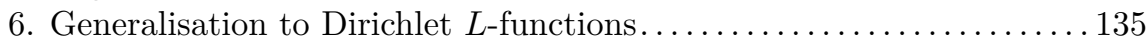

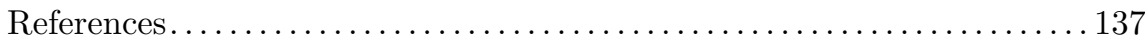

M. Rovinsky - Representations of field automorphism groups ......... 139

1. Translating geometric questions to the language of representation

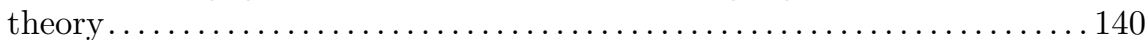

2. From linear to semi-linear representations................... 144

3. Sketch of the proofs and the source of Conjecture $2.7 \ldots \ldots \ldots \ldots 147$

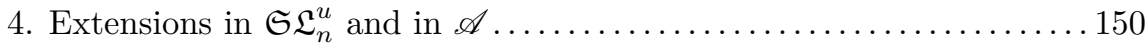

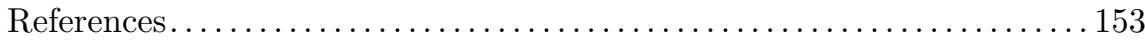

C. BARWICK $-\infty-$ Groupoids, Stacks, and Segal Categories ........... 155 


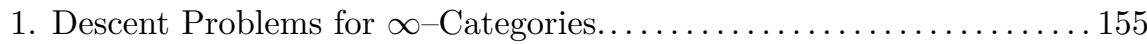

2. Delooping machines and $(\infty, n)$-categories...................... 179

E. LEQUEU - Self-dual normal bases and trace forms of Galois algebras 197 1. Self-dual normal bases............................... 197

2. A reformulation of the problem ............................ 198

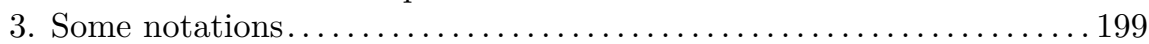

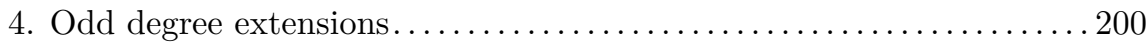

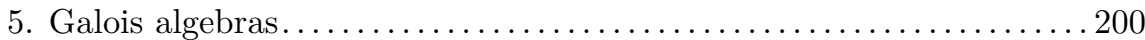

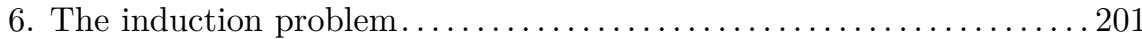

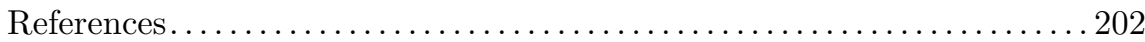

A.-S. Elsenhans \& J. Jahnel - The Diophantine Equation $x^{4}+2 y^{4}=$

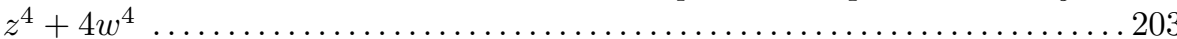

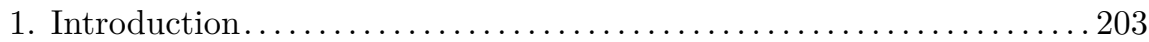

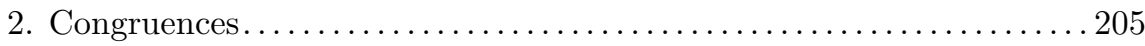

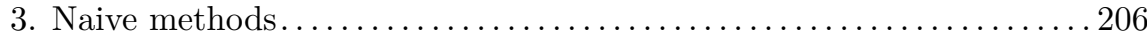

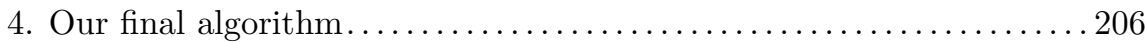

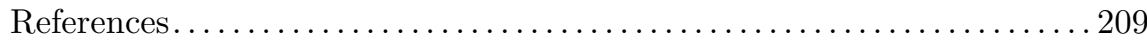





\section{ABSTRACTS}

Notes on differentiable Stacks

Jochen Heinloth..................................... 1

We discuss differentiable stacks and their cohomology. We try to give all necessary definitions, avoiding technical machinery as far as possible. In the last section we focus on the example of $S^{1}$-gerbes and explain the relation to projective (Hilbert-)bundles.

Twisted K-theory and TQFT

UlRich Bunke \& Ingo Schröder........................... 33

The goal of the present paper is the calculation of the equivariant twisted K-theory of a compact Lie group which acts on itself by conjugations, and elements of a TQFT-structure on the twisted K-groups. These results are originally due to D.S. Freed, M.J. Hopkins and C. Teleman. In this paper we redo their calculations in the framework of topological and differentiable stacks. We also show how moduli spaces of flat connections on surfaces give rise to trivializations of twists.

Universality of L-functions

Jörn Steuding.......................................... 81

We survey recent results on the value-distribution of $L$-functions with emphasis on aspects of universality.

Masseyprodukte in der Galoiskohomologie von Zahlkörpern

Denis Vogel............................................ 93 
We study the connection between Massey products and relations in pro- $p$-groups and give an arithmetical example, thereby obtaining a cohomological interpretation of the Rédei symbol.

Finite flat commutative group schemes over complete discrete valuation fields: classification, structural results; application to reduction of Abelian varieties

MikHAil V. BondARKO................................. 99

This is a summary of author's results on finite flat commutative group schemes. The properties of the generic fibre functor are discussed. A complete classification of finite local flat commutative group schemes over mixed characteristic complete discrete valuation rings in terms of their Cartier modules (defined by Oort) is given. We also state several properties of the tangent space of these schemes. These results are applied to the study of reduction of Abelian varieties. A finite $p$-adic semistable reduction criterion is formulated. It looks especially nice in the ordinary reduction case. The plans of the proofs are described.

Toric reduction and tropical geometry

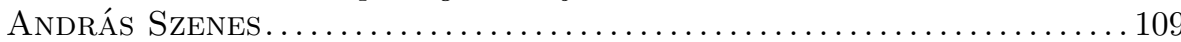

In this note, I review a result obtained in joint work with Michèle Vergne on a duality principle in toric geometry. I will demonstrate the essential points of our work on a concrete example.

A spectral interpretation for the zeros of the Riemann zeta function

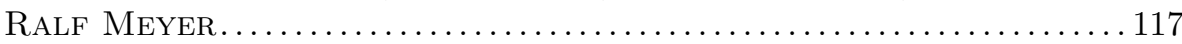

Based on work of Alain Connes, I have constructed a spectral interpretation for zeros of $L$-functions. Here we specialise this construction to the Riemann $\zeta$-function. We construct an operator on a nuclear Fréchet space whose spectrum is the set of non-trivial zeros of $\zeta$. We exhibit the explicit formula for the zeros of the Riemann $\zeta$-function as a character formula.

Representations of field automorphism groups

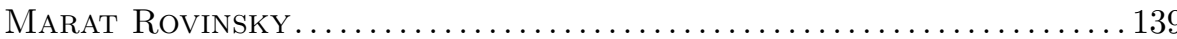

We relate questions in birational algebraic geometry to representation theory. 
$\infty$-Groupoids, Stacks, and Segal Categories

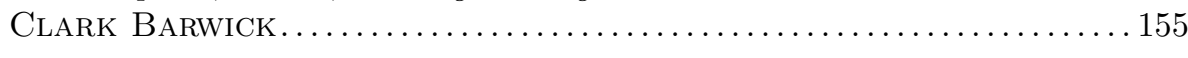

Motivated by descent problems for $K$-theory and derived categories, and inspired by insights in the theory of infinite loop spaces, I introduce a robust theory of $(\infty, n)$-categories and $(\infty, n)$-stacks, and I formulate several important results and conjectures within this framework.

Self-dual normal bases and trace forms of Galois algebras

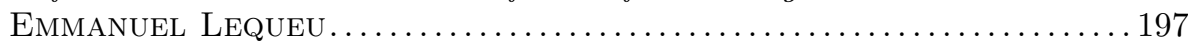

In this note, we present a survey on the problem of the existence of a self-dual normal basis in a finite Galois extension and say how this problem is related to the study of trace forms of Galois algebras.

The Diophantine Equation $x^{4}+2 y^{4}=z^{4}+4 w^{4}$

Andreas-Stephan Elsenhans \& Jörg JAhnel...................... 203

We show that, within the hypercube $|x|,|y|,|z|,|w| \leqslant 2.5 \cdot 10^{6}$, the Diophantine equation $x^{4}+2 y^{4}=z^{4}+4 w^{4}$ admits essentially one and only one non-trivial solution, namely

$$
( \pm 1484801, \pm 1203120, \pm 1169407, \pm 1157520) \text {. }
$$

The investigation is based on a systematic search by computer. 



\section{INTRODUCTION}

This volume contains lecture notes from the seminars

- Number Theory,

- Algebraic Geometry,

- Twisted cohomology theories

which took place at the Mathematics Institute of the University of Göttingen in 2004-2005. They have been arranged in chronological order.

Yuri Tschinkel

June 20, 2005 

Mathematisches Institut, Seminars, (Y. TschINkel, ed.), p. 1-32

Universität Göttingen, 2004-05

\title{
NOTES ON DIFFERENTIABLE STACKS
}

\section{J. Heinloth}

Mathematisches Institut, Universität Göttingen, Bunsenstr. 3-5, D-37073

Göttingen, Germany • E-mail : heinloth@uni-math.gwdg.de

\begin{abstract}
We discuss differentiable stacks and their cohomology. We try to give all necessary definitions, avoiding technical machinery as far as possible. In the last section we focus on the example of $S^{1}$-gerbes and explain the relation to projective (Hilbert-)bundles.
\end{abstract}

\section{Introduction}

These are notes of two lectures given at the Forschungsseminar Bunke-Schick during the Spring term 2004. My task was to explain the notions of stacks and twists. Since this should serve as an introduction to the subject I tried to avoid most of the algebraic language, hoping to make the concept of stacks more understandable. These notes do not claim much originality, all concepts from the theory of algebraic stacks are explained in the book of Laumon and Moret-Bailly [LMB00]. I tried to translate the differentiable setting which is used in $[\mathbf{L T X}]$ and $[\mathbf{F H T}]$ into this language.

The plan of the text is as follows. We start with the example of the stack classifying $G$-bundles, to motivate the abstract definition of stacks. This definition, given in the first section does not look very geometric, therefore we introduce the notion of charts (sometimes called presentations) in the second section. This allows us to define topological and differentiable stacks. In the

November 2004 . 
algebraic setting, this concept was introduced by Deligne and Mumford in their famous article on the irreducibility of the moduli space of curves. Their definition allowed to introduce a lot of geometric notions for stacks and it provided a way of thinking about a differentiable stack as a manifold in which points are allowed to have automorphisms. In the third section we then compare this approach with the groupoid-approach which seems to be better known in topological contexts. The fourth section then defines sheaves, bundles and their cohomology on differentiable stacks. We also provide some easy examples to give an idea of how to do calculations in this setup.

In the last two sections we then give a definition of twists or $S^{1}$-gerbes and we show that they are classified by elements in $H^{2}\left(\ldots, S^{1}\right)$. To compare this with the approach via projective bundles, we then introduce the notion of a local quotient stack, which is used in $[\mathbf{F H T}]$ to give a definition of twisted $K$-theory. For $S^{1}$-gerbes on a local quotient stack we give a construction of a PU-bundle on the stack which defines the gerbe.

\section{Motivation and the first definition of stacks}

The simplest example of a stack is the classifying stack of $G$-bundles: Let $G$ be a topological group. In topology one defines a classifying space $B G$ characterized by the property that for any good space (e.g., CW-Complex):

$\operatorname{Map}(X, B G) /$ homotopy $=\{$ Isom. classes of locally trivial $G$-bundles on $X\}$.

This defines $B G$ uniquely up to homotopy. For finite groups $G$ this space has the additional property, that the homotopy classes of homotopies between two classifying maps are identified with isomorphisms between the corresponding $G$-bundles.

Such a definition of $B G$ is not well suited for algebraic categories, because there a good notion of homotopy is not easy to define. Moreover even in analytic categories the spaces $B G$ usually are infinite dimensional and therefore more difficult to handle.

Regarding the first problem, one could ask the naive question: Why don't we look for a space $B G$ for which $\operatorname{Map}(X, B G)$ is the set of isomorphism classes of $G$-bundles on $X$ ? Of course such a space cannot exist because locally every bundle is trivial, thus the corresponding map should be locally constant, thus constant on connected components of $X$. But not every bundle is globally trivial. 
On the other hand, this argument is somewhat bizarre, because usually $G$-bundles are defined by local data. The problem only arises because we passed to isomorphism classes of bundles.

Thus the first definition of the stack $\underline{B G}$ will be as the (2-)functor assigning to any space $X$ the category of $G$-bundles on $X$. The axioms for such a functor to be a stack will be modeled on the properties of this particular example. Namely the axioms assure that we can glue bundles given on an open covering. This basic example should be held in mind for the following definition of a stack.

Further, to compare this definition with usual spaces one has to keep in mind the Yoneda lemma: Any space/manifold $M$ is uniquely determined by the functor $\operatorname{Map}(, M)$ : Manifolds $\rightarrow$ Sets. This holds in any category (see Lemma 1.3 below).

Therefore, instead of describing the space, we will first consider the corresponding functor and try to find a geometric description afterwards.

Definition 1.1. A stack $\mathscr{M}$ is a $(2-)$ functor

$$
\mathscr{M}: \text { Manifolds } \rightarrow \text { Groupoids } \subset \text { Cat },
$$

i.e.:

- for any manifold $X$ we get a category $\mathscr{M}(X)$ in which all morphisms are isomorphisms, and

- for any morphism $f: Y \rightarrow X$ we get a functor

$$
f^{*}: \mathscr{M}(X) \rightarrow \mathscr{M}(Y)
$$

( $i d^{*}$ has to be the identity),

- for any $Z \stackrel{g}{\longrightarrow} Y \stackrel{f}{\longrightarrow} X$ a natural transformation $\Phi_{f, g}: g^{*} f^{*} \cong(g \circ f)^{*}$, which is associative whenever we have 3 composable morphisms.

For a stack $\mathscr{M}$ we require the 2 -functor to have glueing-properties (to make these more readable ${ }^{(1)}$, we write $\left.\right|_{U}$ instead of $j^{*}$, whenever $U \stackrel{j}{\hookrightarrow} X$ is an open embedding):

1. We can glue objects: Given an open covering $U_{i}$ of $X$, objects $P_{i} \in \mathscr{M}\left(U_{i}\right)$ and isomorphisms $\varphi_{i j}:\left.\left.P_{i}\right|_{U_{i} \cap U_{j}} \rightarrow P_{j}\right|_{U_{i} \cap U_{j}}$ which satisfy the cocycle condition on threefold intersections $\varphi_{j k} \circ \varphi_{i j}=\left.\varphi_{i k}\right|_{U_{i} \cap U_{j} \cap U_{k}}$ there is an object $P \in \mathscr{M}(X)$ together with isomorphisms $\varphi_{i}:\left.P\right|_{U_{i}} \rightarrow P_{i}$ such that $\varphi_{i j}=\varphi_{j} \circ \varphi_{i}^{-1}$.

\footnotetext{
${ }^{(1)} \mathrm{cf}$. first remark below
} 
2. We can glue morphisms: Given objects $P, P^{\prime} \in \mathscr{M}(X)$, an open covering $U_{i}$ of $X$ and isomorphisms $\varphi_{i}:\left.\left.P\right|_{U_{i}} \rightarrow P^{\prime}\right|_{U_{i}}$ such that $\left.\varphi_{i}\right|_{U_{i} \cap U_{j}}=\left.\varphi_{j}\right|_{U_{i} \cap U_{j}}$, then there is a unique $\varphi: P \rightarrow P^{\prime}$ such that $\varphi_{i}=\left.\varphi\right|_{U_{i}}$.

\section{Remarks 1.2.}

1. Formally the glueing conditions make use of the natural transformations for the inclusions $U_{i} \cap U_{j} \hookrightarrow U_{i} \hookrightarrow X$, this is not visible above, because of our notation $\left.\right|_{U_{i} \cap U_{j}}$. For example write $U_{i j k}=U_{i} \cap U_{j} \cap U_{k}$, denote $j_{i j k, i j}: U_{i j k} \rightarrow$ $U_{i j}, j_{i j, i}: U_{i j} \rightarrow U_{i}, j_{i j k, i}: U_{i j k} \rightarrow U_{i}$ the inclusions. Then we have natural transformations

$$
\Phi_{i j k, i j, i}: j_{i j k, i j}^{*} j_{i j, i}^{*} \rightarrow j_{i j k, i}^{*}
$$

In the condition to glue objects

$$
\left.\left.\varphi_{j k}\right|_{U_{i j k}} \circ \varphi_{i j}\right|_{U_{i j k}}=\left.\varphi_{i k}\right|_{U_{i j k}}
$$

we would formally have to replace $\left.\varphi_{i j}\right|_{U_{i j k}}$ by the composition:

$$
j_{i j k, i}^{*} P_{i} \stackrel{\Phi_{i j k, i j, i}}{\longrightarrow} j_{i j k, i j}^{*} j_{i j, i}^{*} P_{i} \stackrel{j_{i j k, i j}^{*} \varphi_{i j}}{\longrightarrow} j_{i j k, i j}^{*} j_{i j, j}^{*} P_{j} \stackrel{\Phi_{i j k, i j, j}^{-1}}{\longrightarrow} j_{i j k, j}^{*} P_{j}
$$

and similarly for the other maps, but this makes the condition hard to read.

2. Our functor $\underline{B G}$, assigning to any manifold the category of $G$-bundles is a stack.

3. We could replace manifolds by topological spaces in the above definition. This is usually phrased as giving a stack over manifolds and a stack over topological spaces respectively.

4. Stacks form a 2-category: Morphisms $F: \mathscr{M} \rightarrow \mathscr{N}$ of stacks are given by a collection of functors $F_{X}^{*}: \mathscr{N}(X) \rightarrow \mathscr{M}(X)$ and, for any $f: X \rightarrow Y$, a natural transformation $F_{f}: f^{*} F_{X}^{*} \cong F_{Y}^{*} f^{*}$. Thus morphisms of stacks form a category, morphisms between morphisms of stacks (i.e., natural transformations $\varphi_{X}: F_{X} \rightarrow G_{X}$ satisfying $\left.G_{f} \circ \varphi_{X}=\varphi_{Y} \circ F_{f}\right)$ are written as $\stackrel{\mathscr{M} \longrightarrow \mathscr{N}}{\longrightarrow}$. Note that all 2-morphisms are invertible, since all maps in the categories $\mathscr{M}(X)$ and $\mathscr{N}(X)$ are invertible.

5. The inclusion Sets $\rightarrow$ Groupoids (associating to each set the category whose objects are elements of the set and the only morphisms are identities) is a full embedding. By the Yoneda lemma we know that the functor Top $\rightarrow$ Functors is a full embedding, thus we get a full embedding Top $\rightarrow$ Stacks. This embedding assigns to a space $X$ the stack $\underline{X}$ defined as $\underline{X}(Y)=\operatorname{Map}(Y, X)$, this is a stack, since maps can be glued, pull-back functors are given by the composition of maps.

6. Grothendieck topology of maps with local sections: Bundles, (in fact any stack), satisfy a better glueing condition, namely we do not need that the 
$j: U_{i} \hookrightarrow X$ are injective. Whenever we have a map $\cup U_{i} \stackrel{p}{\longrightarrow} X$ such that $p$ has local sections (i.e., for all points $x \in X$ there is a neighborhood $U_{x}$ and a section $s: U_{x} \rightarrow \cup U_{i}$ of $p$, in particular $p$ is surjective). Then the glueing condition also holds, if we replace $U_{i} \cap U_{j}$ by the fibered product $U_{i} \times_{X} U_{j}$. We say that the stack is a stack for the local-section-topology. This point of view will be important to define charts for stacks.

(If we wanted to stay in the category of manifolds instead of topological spaces, we should require the map $p$ to be a submersion, in order to have fibered products.)

The following lemma shows, that with the above definition of $\underline{B G}$ we really get a classifying object for $G$-bundles:

Lemma 1.3 (Yoneda lemma for stacks). Let $\mathscr{M}$ be a stack (defined for manifolds or topological spaces). For any space $X$ denote by $\underline{X}$ the associated stack (i.e., $\underline{X}(Y)=\operatorname{Map}(Y, X))$. Then there is a canonical equivalence of categories: $\mathscr{M}(X) \cong \operatorname{Mor}_{\text {Stacks }}(\underline{X}, \mathscr{M})$.

Proof. Given $P \in \mathscr{M}(X)$ we define a morphism $F_{P}: \underline{X} \rightarrow \mathscr{M}$ by

$$
\underline{X}(Y) \ni(Y \stackrel{f}{\longrightarrow} X) \mapsto f^{*} P \in \mathscr{M}(Y) .
$$

For any isomorphism $\varphi: P \rightarrow P^{\prime}$ in $\mathscr{M}(X)$ we define a natural transformation $A_{\varphi}: F_{P} \rightarrow F_{P^{\prime}}$ by $f^{*} \varphi: f^{*} P \rightarrow f^{*} P^{\prime}$. Conversely, given a morphism $F$ : $\underline{X} \rightarrow \mathscr{M}$ we get an object $P_{F}:=F\left(i d_{X}\right) \in \mathscr{M}(X)$, any automorphism $F \rightarrow F$ defines an isomorphism of $P_{F}$.

One checks that the composition of these constructions is equivalent to the identity functor.

Remark 1.4. Will often write $X$ instead of $\underline{X}$.

Example 1.5 (Quotient stacks). Let $G$ be a Lie group acting on a manifold $X$ via act $: G \times X \rightarrow X$. We define the quotient stack $[X / G$, act $]$ (or simply $[X / G])$ as

$$
[X / G, \operatorname{act}](Y):=\langle(P \stackrel{p}{\longrightarrow} Y, P \stackrel{f}{\longrightarrow} X)| P \rightarrow Y \text { a G-bundle, } f \text { G-equivariant }\rangle .
$$

Morphisms of objects are $G$-equivariant isomorphisms.

\section{Remarks 1.6.}

1. For $G$ acting trivially on $X=p t$ the quotient $[p t / G]$ is the stack $\underline{B G}$ classifying $G$-bundles. 
2. If $G$ acts properly and freely, i.e. $X \rightarrow X / G$ is a $G$-bundle, then $[X / G] \cong$ $X / G$, because any $f: P \rightarrow X$ defines a map on the quotient $P / G=Y \rightarrow$ $X / G$ and the canonical morphism $P \rightarrow Y \times_{X / G} X$ is then an isomorphism of $G$-bundles.

\section{Geometry I: Charts}

To translate geometric concepts to the (2-)category of stacks, Deligne and Mumford introduced a notion of charts for stacks.

In our example $\underline{B G}$ the Yoneda-lemma 1.3 shows that the trivial bundle on a point $p t$ defines a map $p t \rightarrow \underline{B G}$. By the same lemma any $X \stackrel{f_{P}}{\longrightarrow} B G$ is given by a bundle $P \rightarrow X$. Therefore, if we take a covering $U_{i} \rightarrow X$ on which the bundle is trivial, then $\left.f_{P}\right|_{U_{i}}$ factors through $p t \rightarrow B G$. In particular, this trivial map is in some sense surjective (see Definition 2.3 for a precise definition, we will say that this map has local sections)!

Even more is true: First note that the (2-)category of stacks has fibered products:

Definition 2.1. Given a diagram of morphisms of stacks:

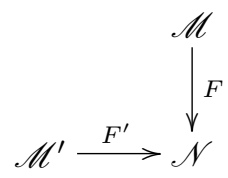

we define the fibered product $\mathscr{M} \times \mathscr{N} \mathscr{M}^{\prime}$ to be the stack given by:

$$
\mathscr{M} \times \mathscr{N} \mathscr{M}^{\prime}(X):=\left\langle\left(f, f^{\prime}, \varphi\right) \mid f: X \rightarrow \mathscr{M}, f^{\prime}: X \rightarrow \mathscr{M}^{\prime}, \varphi: F \circ f \Rightarrow F^{\prime} \circ f^{\prime}\right\rangle .
$$

Morphisms $\left(f, f^{\prime}, \varphi\right) \rightarrow\left(g, g^{\prime}, \psi\right)$ are pairs of morphisms

$$
\left(\varphi_{f, g}: f \rightarrow g, \varphi_{f^{\prime}, g^{\prime}}: f^{\prime} \rightarrow g^{\prime}\right)
$$

such that

$$
\psi \circ F\left(\varphi_{f, g}\right)=F^{\prime}\left(\varphi_{f^{\prime}, g^{\prime}}\right) \circ \varphi .
$$

(We will use brackets \langle\rangle as above to denote groupoids instead of sets \{\} )

Remark 2.2. This defines a stack, because objects of $\mathscr{M}, \mathscr{N}$ glue and morphisms of $\mathscr{N}, \mathscr{M}, \mathscr{M}^{\prime}$ glue. 
We calculate the fibered product in our example above: Given

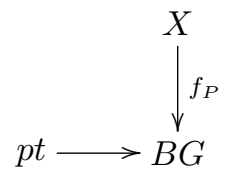

the fibered product $p t \times_{B G} X$ as the stack given by:

$$
\begin{aligned}
p t \times_{B G} X(Y) & =\left\langle\begin{array}{l}
Y \stackrel{g}{\longrightarrow} X\rangle \\
\end{array}\right. \\
& \left.=\langle(g, \varphi)| g: Y \rightarrow X \text { and } \varphi: g^{*} P \stackrel{\cong}{\longrightarrow} G \times Y\right\rangle \\
& \cong\left\{(g, s) \mid g: Y \rightarrow X \text { and } s: Y \rightarrow g^{*} P \text { a section }\right\} \\
& \cong\{\tilde{g}: Y \rightarrow P\}=P(Y)
\end{aligned}
$$

The first $\cong$ notes that to give a trivialization of $g^{*} P$ is the same as to give a section of $g^{*} P$, in particular the category defined above is equivalent to a set. The second $\cong$ assigns to $\tilde{g}$ the composition of $\tilde{g}$ with the projection $P \rightarrow X$ and the section induced by $\tilde{g}$.

By the last description, we get an equivalence $p t \times_{B G} X \cong \underline{P}$, i.e., $p t \rightarrow B G$ is the universal bundle over $B G$.

Definition 2.3. A stack $\mathscr{M}$ is called a topological stack (resp. differentiable stack) if there is a space (resp. manifold) $X$ and a morphism $p: X \rightarrow \mathscr{M}$ such that:

1. For all $Y \rightarrow \mathscr{M}$ the stack $X \times \mathscr{M} Y$ is a space (resp. manifold).

2. $p$ has local sections (resp. is a submersion), i.e., for all $Y \rightarrow \mathscr{M}$ the projection $Y \times \mathscr{M} X \rightarrow Y$ has local sections (resp. is a submersion).

The map $X \rightarrow \mathscr{M}$ is then called a covering or an atlas of $\mathscr{M}$ (in the localsection-topology).

The first property is very important, it therefore gets an extra name:

Definition 2.4. A morphism of stacks $F: \mathscr{M} \rightarrow \mathscr{N}$ is called representable if for any $Y \rightarrow \mathscr{N}$ the fibered product $\mathscr{M} \times \mathscr{N} Y$ is a stack which is equivalent to a topological space.

This definition is the requirement that the fibres of a morphism should be topological spaces and not just stacks. We will see later, that for topological stacks this condition is equivalent to the condition that the morphism $F$ is 
injective on automorphism groups of objects. The easiest example of such a map is the map $p t \rightarrow B G$ we have seen above. The easiest example of a map which is not representable is the map $B G \rightarrow p t$ forgetting everything (take $Y=p t)$.

Example 2.5 (Quotient-stacks). The example of quotients by group actions $[X / G]$ are topological stacks (resp. differentiable, if $X, G$ are smooth). An atlas is given by the quotient map $X \rightarrow[X / G]$, defined by the trivial $G$-bundle $G \times X \rightarrow X$, the action map $G \times X \stackrel{\text { act }}{\longrightarrow} X$ is $G$-equivariant.

Just as in the case of $G$-bundles one shows that for any $Y \rightarrow[X / G]$ given by a $G$-bundle $P \rightarrow Y$ there is a canonical isomorphism $Y \times_{[X / G]} X \cong P$ (the argument is given a second time in Lemma 3.1 below).

Some easy properties of representable morphisms are:

\section{Lemma 2.6.}

1. (Composition) If $F: \mathscr{K} \rightarrow \mathscr{M}$ and $G: \mathscr{M} \rightarrow \mathscr{N}$ are representable, then $F \circ G$ is representable.

2. (Pull-back) If $F: \mathscr{M} \rightarrow \mathscr{N}$ is representable, and $G: \mathscr{M}^{\prime} \rightarrow \mathscr{N}$ is arbitrary then the projection $\mathscr{M}^{\prime} \times \mathscr{N} \mathscr{M} \rightarrow \mathscr{M}^{\prime}$ is representable.

3. (Locality) A morphism $F: \mathscr{M} \rightarrow \mathscr{N}$ of topological stacks is representable if and only if for one atlas $Y \rightarrow \mathscr{N}$ the product $\mathscr{M} \times \mathscr{N} Y \rightarrow \mathscr{M}$ is again an atlas.

4. If $\mathscr{M}$ is a topological stack, then for any two morphisms $f_{i}: Y_{i} \rightarrow \mathscr{M}$ the fibered product $Y_{1} \times \mathscr{M} Y_{2}$ is again a topological space.

Proof. For the first claim note that $Y \times \mathscr{N} \mathscr{K} \cong(Y \times \mathscr{N} \mathscr{M}) \times \mathscr{M} \mathscr{K}$, the latter is a space by assumption.

The second is similar: $Y \times \mathscr{M}^{\prime}\left(\mathscr{M}^{\prime} \times \mathscr{N} \mathscr{M}\right) \cong Y \times \mathscr{N} \mathscr{M}$.

If $\mathscr{M} \rightarrow \mathscr{N}$ is representable, then $Y \times \mathscr{N} \mathscr{M}$ is a topological space and for any $T \rightarrow \mathscr{M}$ we have $T \times \mathscr{M}(Y \times \mathscr{N} \mathscr{M})=T \times \mathscr{N} Y \rightarrow Y$ has local sections.

On the other hand, if $Y \times \mathscr{N} \mathscr{M} \rightarrow \mathscr{M}$ is an atlas, then for all $T \rightarrow \mathscr{N}$ which factor through $T \rightarrow Y \rightarrow \mathscr{N}$ the pull back $T \times \mathscr{N} \mathscr{M}$ is again a space. For an arbitrary $T \rightarrow \mathscr{N}$ the projection $Y \times \mathscr{N} T \rightarrow T$ has local sections by assumption. This shows, that the fibered product $T \times \mathscr{N} \mathscr{M}$ is a stack which is equivalent to a functor, and that there is a covering $U_{i}$ of $T$, such that $U_{i} \times \mathscr{N} \mathscr{M}$ is a space. Now functoriality of fibered products assures, that these spaces can be glued, thus $T \times \mathscr{N} \mathscr{M}$ is a space.

For the last statement, note that $Y_{1} \times \mathscr{M} Y_{2} \cong\left(Y_{1} \times Y_{2}\right) \times \mathscr{M} \times \mathscr{M} \mathscr{M}$ where the map $\Delta: \mathscr{M} \rightarrow \mathscr{M} \times \mathscr{M}$ is the diagonal map. Thus the assumption may be 
rephrased as "the diagonal $\Delta: \mathscr{M} \rightarrow \mathscr{M} \times \mathscr{M}$ is representable" and then the claim follows from (3).

Remark 2.7. In the last statement of the lemma, there is a natural map $Y_{1} \times \mathscr{M} Y_{2} \rightarrow Y_{1} \times Y_{2}$, but in general this is not an embedding, thus the diagonal $\mathscr{M} \rightarrow \mathscr{M} \times \mathscr{M}$ is not an embedding in general.

One should also note that the fibered product $Y_{1} \times \mathscr{M} Y_{2}$ represents the functor of maps $T \rightarrow Y_{1} \times Y_{2}$ together with an isomorphism of the two pull backs of the objects $p_{i}^{*}\left(Y_{i} \rightarrow \mathscr{M}\right)$, therefore it is sometimes denoted

$$
\operatorname{Isom}\left(Y_{1} \stackrel{f_{1}}{\longrightarrow} \mathscr{M}, Y_{2} \stackrel{f_{2}}{\longrightarrow} \mathscr{M}\right)
$$

or simply $I \operatorname{som}\left(f_{1}, f_{2}\right)$. In particular one sees that the automorphisms of a map $f: Y \rightarrow \mathscr{M}$ are given by sections of the map $A u t(f):=\left(Y \times{ }_{\mathscr{M}} Y\right) \times_{Y \times Y} Y \rightarrow Y$, because a map from a space $T$ to $A u t(f)$ is the same as a map $s: T \rightarrow Y$ together with an isomorphism $\varphi: f \circ s \Rightarrow f \circ s$.

Any property of maps which can be checked on submersions can now be defined for representable morphisms of differentiable stacks, simply requiring that the property holds for one atlas:

Definition 2.8. A representable morphism $\mathscr{M} \rightarrow \mathscr{N}$ is an open embedding, (resp. closed embedding, submersion, proper, ...) if for one (equivalently any) atlas $Y \rightarrow \mathscr{N}$ the map $\mathscr{M} \times \mathscr{N} Y \rightarrow \mathscr{Y}$ is an open embedding (resp. closed embedding, submersion, proper, ...).

Note that if $\mathscr{M}$ and $\mathscr{N}$ are spaces then every map is representable and we get the usual notion of open embedding, etc.

In particular, this definition gives us a notion of open and closed substacks.

Example 2.9. For quotient-stacks $[X / G]$ open and closed substacks are given by open and closed $G$-equivariant subspaces $Y \hookrightarrow X$, which define embeddings $[Y / G] \hookrightarrow[X / G]$.

Properties that can be checked on coverings of the source of a map (i.e., to have local sections, or in the differentiable category to be smooth or submersive) can even be defined for any morphism of stacks:

Definition 2.10. An arbitrary morphism $\mathscr{M} \rightarrow \mathscr{N}$ of differentiable (resp. topological) stacks is smooth (or a submersion) (resp. has local sections), if for one (equivalently any) atlas $X \rightarrow \mathscr{M}$ the composition $X \rightarrow \mathscr{N}$ is smooth (or a submersion) (resp. has local sections), i.e., for one (equivalently any) atlas 
$Y \rightarrow \mathscr{N}$ the fibered product $X \times \mathscr{N} Y \rightarrow Y$ is smooth (or a submersion) (resp. has local sections).

The equivalence of the condition to be satisfied for one or for any atlas is proved as in Lemma 2.6.

Note that we can glue morphisms of stacks, i.e., given an atlas $X \rightarrow \mathscr{M}$ and a morphism $\mathscr{M} \rightarrow \mathscr{N}$ of topological or differentiable stacks we get an induced morphism $X \rightarrow \mathscr{N}$ together with an isomorphism of the two induced morphisms $X \times \mathscr{M} X \longrightarrow \mathscr{N}$, which satisfies the cocycle condition on $X \times \mathscr{M}$ $X \times \mathscr{M} X$.

Conversely, given $f: X \rightarrow \mathscr{N}$ together with an isomorphism $p_{1} \circ f \Rightarrow p_{2} \circ f$ of the two induced maps $X \times \mathscr{M} X \rightarrow \mathscr{N}$, which satisfies $p_{23}^{*} \varphi \circ p_{12}^{*} \varphi=p_{13}^{*} \varphi$ on $X^{\times{ }^{3} \mathscr{M}}$ we get a morphism $\mathscr{M} \rightarrow \mathscr{N}$ as follows: For any $T \rightarrow \mathscr{M}$ we get a map with local sections $X \times \mathscr{M} T \rightarrow T$ and a map $X \times \mathscr{M} T \rightarrow \mathscr{N}$ together with a glueing data on $X \times \mathscr{M} X \times \mathscr{M} T=(X \times \mathscr{M} T) \times_{T}\left(X \times \mathscr{M}^{T}\right)$, and by the glueing condition for stacks this canonically defines an element in $\mathscr{N}(T)$.

In particular, a morphism $\mathscr{M} \rightarrow \underline{B G}$ is the same as a $G$-bundle on an atlas $X$ together with a glueing datum on $X \times \mathscr{M} X$ satisfying the cocycle condition on $X \times \mathscr{M} X \times \mathscr{M} X$. If $\mathscr{M}=[X / H]$ is a quotient stack then $X \times \mathscr{M} X \cong H \times X$, thus this is the same as an $H$-equivariant bundle on $X$.

More generally, for any class of objects which satisfy descent, i.e., which can be defined locally by glueing data, we can define the corresponding objects over stacks to be given as a glueing-data on one atlas. For example vector bundles, Hilbert-bundles, smooth fibrations.

Definition 2.11. A $G$-bundle over a stack $\mathscr{M}$ is given by a $G$-bundle $\mathscr{P}_{X}$ over an atlas $X \rightarrow \mathscr{P}$ together with an isomorphism of the two pull-backs of $p_{1}^{*} \mathscr{P}_{X} \rightarrow p_{2}^{*} \mathscr{P}_{X}$ on $X \times \mathscr{M} X$ satisfying the cocycle condition on $X \times \mathscr{M} X \times \mathscr{M} X$.

The same definition applies to vector bundles, Hilbert bundles, locally trivial fibrations with fiber $F$.

Remark 2.12. Note that for any $f: T \rightarrow \mathscr{M}$ (in particular for any atlas) this datum defines a $G$-bundle $P_{T, f} \rightarrow T$, because by definition $X \times \mathscr{M} T \rightarrow T$ has local sections, and we can pull-back the glueing datum to

$$
(X \times \mathscr{M} X \times \mathscr{M} T) \cong(X \times \mathscr{M} T) \times_{T}(X \times \mathscr{M} T) .
$$

Therefore this automatically defines a differentiable/topological stack $\mathscr{P} \stackrel{p}{\longrightarrow}$ $\mathscr{M}$ (and $p$ is representable) via:

$$
\mathscr{P}(T)=\left\langle\left(f: T \rightarrow \mathscr{M}, s: T \rightarrow P_{T, f} \text { a section }\right)\right\rangle .
$$


An atlas of this stack is given by $\left(\mathscr{P}_{X}, s: \mathscr{P}_{X} \stackrel{\text { diag }}{\longrightarrow} \mathscr{P}_{X} \times_{X} P_{X}\right)$. The multiplication map glues, therefore this stack also carries a natural morphism $G \times \mathscr{P} \rightarrow \mathscr{P}$.

Remark 2.13. This shows that universal bundles on stacks classifying $G$-bundles or other geometric objects exist automatically. Further, since we can glue morphisms of stacks the classifying stack will also classify $G$-bundles on stacks.

Remark 2.14. Note further, that given a $G$-bundle $\mathscr{P}$ on a stack $\mathscr{M}$ and a map $f: T \rightarrow \mathscr{M}$ the glueing datum for the two pull backs of $P_{T, f}$ to $T \times \mathscr{M} T$ defines an action of $\operatorname{Aut}(s)$ on $f^{*} \mathscr{P}=P_{T, f}$.

The notion of a $G$-bundle could be defined directly in the language of stacks. These definitions tend to get clumsy, because one has to take care of automorphisms:

Let $G$ be a Lie group, a locally trivial $G$-bundle over an analytic stack $\mathscr{M}$ is a stack $\mathscr{P}$ together with a representable morphism $\mathscr{P} \stackrel{p}{\longrightarrow} \mathscr{M}$, an action $G \times \mathscr{P} \stackrel{\text { act }}{\longrightarrow} \mathscr{P}$ together with an isomorphism $\varphi: p \circ$ act $\cong p$, such that act is simply transitive on the fibers of $p$, an isomorphism $\varphi_{2}$ making the diagram

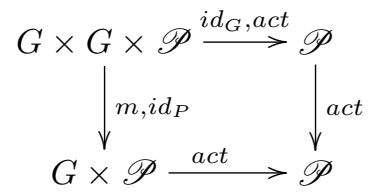

commute, such that in the induced isomorphisms in the associativity diagram coincide. Further, there has to be a two morphism making the diagram $\mathscr{P} \stackrel{e, i d}{\longrightarrow} G \times \mathscr{P}$ commute, compatible with multiplication. Finally to make a<smiles>CC(C)C</smiles>

bundle locally trivial there should exist an atlas $X \rightarrow \mathscr{M}$ such that the induced bundle $\mathscr{P} \times \mathscr{M} X \rightarrow X$ is trivial.

Claim. The two notions of $G$-bundles coincide. (We will never use this.)

Example 2.15. Once more, note that $p t \rightarrow[p t / G]$ is a $G$-bundle over $p t$. The action map $G \times p t \rightarrow p t$ is trivial. And we note that $p t \rightarrow[p t / G]$ corresponds to the trivial bundle, thus a trivialization of this bundle induces canonical isomorphisms $\varphi$. 


\section{Topological stacks as topological groupoids}

We can generalize the example of quotients by group actions as follows: Given an atlas $X \rightarrow \mathscr{M}$, the two projections $X \times \mathscr{M} X \gtreqless X$ define the source an target morphisms of a groupoid, the diagonal is the identity, interchanging the factors the inverse and the composition is given by the projection to the first and third factor of

$$
X \times \mathscr{M} X \times \mathscr{M} X \cong(X \times \mathscr{M} X) \times_{X}(X \times \mathscr{M} X) \rightarrow X \times \mathscr{M} X .
$$

We will denote this groupoid by $X_{\bullet}$.

Conversely, any groupoid $\Gamma_{1} \gtreqless \Gamma_{0}$ defines a topological stack:

$$
\left[\Gamma_{0} / \Gamma_{1}\right](Y):=\left\langle\left(P \stackrel{p}{\longrightarrow} Y, P \stackrel{f}{\longrightarrow} \Gamma_{0}\right) \text { a locally trivial } \Gamma-\text { bundle }\right\rangle
$$

Recall that a locally trivial $\Gamma$-bundle is a diagram

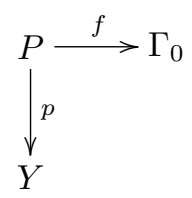

together with an action $\Gamma_{1} \times_{\Gamma_{0}} P \rightarrow \Gamma_{0}$ which is equivariant with respect to composition of morphisms in $\Gamma_{1}$, such that there is a covering $U \rightarrow Y$ and maps $f_{i}: U \rightarrow \Gamma_{0}$ such that $\left.P\right|_{U} \cong f_{i}^{*} \Gamma_{\text {}}$. Note that such a trivialization is the same as a section $U \rightarrow P$ (obtained from the identity section of $\Gamma$ ).

Since we can glue $\Gamma$-bundles this is a stack. As in the case of quotients we have:

Lemma 3.1. The trivial $\Gamma$-bundle $\Gamma_{1} \rightarrow \Gamma_{0}$ induces a map $\Gamma_{0} \stackrel{\pi}{\longrightarrow}\left[\Gamma_{0} / \Gamma_{1}\right]$ which is an atlas for $\left[\Gamma_{0} / \Gamma_{1}\right]$, the map $\pi$ is the universal $\Gamma$-bundle over $\left[\Gamma_{0} / \Gamma_{1}\right]$. The groupoids $\Gamma$ and $\Gamma_{0, \bullet}$ are canonically isomorphic.

Proof. We only need to show, that for any $Y \stackrel{f_{P}}{\longrightarrow}\left[\Gamma_{0} / \Gamma_{1}\right]$ given by a bundle $P$, there is a canonical isomorphism $P \stackrel{\cong}{\longrightarrow} \Gamma_{0} \times_{\left[\Gamma_{0} / \Gamma_{1}\right]} Y$. This is seen as before:

$$
\begin{aligned}
\left(\Gamma_{0} \times_{\left[\Gamma_{0} / \Gamma_{1}\right]} Y\right)(T) & \cong\left\langle\left(T \stackrel{f}{\longrightarrow} Y, T \stackrel{g}{\longrightarrow} \Gamma_{0}, \varphi: f_{P} \circ f \rightarrow \pi \circ g\right\rangle\right. \\
& \cong\left\langle\left(f, g, \varphi: f^{*} P \cong g^{*} \Gamma_{1}\right)\right\rangle \\
& \cong\left\{(f, \tilde{f}: T \rightarrow P) \mid p r_{Y} \circ \tilde{f}=f\right\} \\
& \cong\{\tilde{f}: T \rightarrow P\}=P(T) .
\end{aligned}
$$


Next one wants to know, whether two groupoids $\Gamma_{\bullet}, \Gamma_{\bullet}^{\prime}$ define isomorphic stacks. From the point of view of atlases this is easy: Given two atlases

$$
X \stackrel{p}{\longrightarrow} \mathscr{M}, X^{\prime} \stackrel{p^{\prime}}{\longrightarrow} \mathscr{M}
$$

we get another atlas refining both, namely

$$
X \times \mathscr{M} \times X^{\prime} \rightarrow X \rightarrow \mathscr{M}
$$

is again an atlas (since both maps are representable and have local sections, the same is true for the composition).

Furthermore $X \times \mathscr{M} X^{\prime} \rightarrow X$ is a locally trivial $X_{\bullet}^{\prime}$ bundle. This shows:

Lemma 3.2. Two groupoids $\Gamma_{\bullet}, \Gamma_{\bullet}^{\prime}$ define isomorphic stacks if and only if there is a groupoid $\Gamma_{\bullet}^{\prime \prime}$ which is a left $\dot{\Gamma}_{\bullet}$ bundle over $\Gamma_{0}^{\prime}$ and a right $\Gamma_{\bullet}^{\prime}$ bundle over $\Gamma_{0}$ such that both actions commute.

Example 3.3. If we have a subgroup $H \subset G$ acting on a space $X$, then $[X / H] \cong\left[X \times{ }^{H} G / G\right]$, since the maps $X \leftarrow X \times G \rightarrow X \times{ }^{H} G$ define a $G$-bundle over $X$ and an $H$-bundle over $X \times{ }^{H} G$.

Similarly, if $H \subset G$ is a normal subgroup, acting freely on $X$, such that $X \rightarrow X / H$ is a principal $H$-bundle, then $[X / G] \cong[(X / H) /(G / H)]$, because $G \times{ }^{H} X$ is a $G / H$-bundle over $X$ and a $G$-bundle over $X / H$.

Finally we can identify morphisms of stacks in terms of groupoids, if the morphism is a submersion, then in $[\mathbf{L T X}]$ these are called generalized homomorphisms.

Given a morphism $\mathscr{M} \stackrel{f}{\longrightarrow} \mathscr{N}$ of topological stacks, and atlases $X \rightarrow$ $\mathscr{M}, Y \rightarrow \mathscr{N}$ we can form the fibered product $X \times_{\mathscr{N}} Y \rightarrow X$. Since $\mathscr{N} \rightarrow Y$ is a locally trivial $Y_{\bullet}$ bundle, this is a (right) $Y_{\bullet}$ bundle as well. Furthermore, since the map $X \rightarrow \mathscr{N}$ factors through $\mathscr{M}$ we also get a $X \bullet$ (left) action on $X \times \mathscr{N} Y$. Note that (by definition) the map $X \times_{\mathscr{N}} Y \rightarrow Y$ is a submersion if and only if $\mathscr{M} \rightarrow \mathscr{N}$ is a submersion.

Conversely, suppose we are given $X \leftarrow P \rightarrow Y$, together with commuting actions of $X_{\bullet}$ and $Y_{\bullet}$ on $P$, such that $P$ is a locally trivial $Y_{\bullet}$ bundle over $X$. Then the $X_{\bullet}$ action on $P$ is a descent datum for the $Y_{\bullet}$-bundle, which defines a $Y_{\bullet}$ bundle over $\mathscr{M}$, thus a morphism $\mathscr{M} \rightarrow \mathscr{N}$.

Of course, the simplest case of this is the most useful, namely a morphism of groupoids $X_{\bullet} \rightarrow Y_{\bullet}$ induces a morphism of the associated quotient stacks, ( $P$ as above is then obtained by pulling back $Y_{\bullet}$ to $X=X_{0}$ ). 


\section{Geometry II: Sheaves, cohomology, tangent spaces, dimension, normal bundles}

Given a representable submersion $\mathscr{M} \rightarrow \mathscr{N}$ we define the dimension of the fibers rel.dim $(\mathscr{M} / \mathscr{N})$ as the dimension of the fibers of $\mathscr{M} \times \mathscr{N} Y \rightarrow Y$ for any $Y \rightarrow \mathscr{N}$. This is well defined, because the relative dimension does not change under pull-backs.

Given an analytic stack $\mathscr{M}$ define its dimension by choosing an atlas $X \rightarrow \mathscr{M}$ and defining $\operatorname{dim} \mathscr{M}:=\operatorname{dim}(X)-$ rel.dim$(X / \mathscr{M})$. This is independent of the atlas (check this for a submersion $X^{\prime} \rightarrow X \rightarrow \mathscr{M}$ ).

Definition 4.1. A sheaf $\mathscr{F}$ on a stack is a collection of sheaves $\mathscr{F}_{X \rightarrow \mathscr{M}}$ for any $X \rightarrow \mathscr{M}$, together with, for any triangle

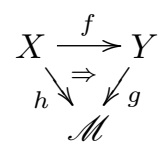

with an isomorphism $\varphi: g \circ f \rightarrow h$, a morphism of sheaves $\Phi_{f, \varphi}: f^{*} \mathscr{F}_{Y \rightarrow \mathscr{M}} \rightarrow$ $\mathscr{F}_{X \rightarrow \mathscr{M}}$, compatible for $X \rightarrow Y \rightarrow Z$ (we often write $\Phi_{f}$ instead of $\Phi_{f, \varphi}$ ). Such that $\Phi_{f}$ is an isomorphism, whenever $f$ is an open covering.

The sheaf $\mathscr{F}$ is called cartesian if all $\Phi_{f, \varphi}$ are isomorphisms.

\section{Remarks 4.2.}

1. Instead of giving sheaves $\mathscr{F}_{X \rightarrow \mathscr{M}}$ for all $X \rightarrow \mathscr{M}$, we could as well only give the global sections $\mathscr{F}_{X \rightarrow \mathscr{M}}(X)$, together with restriction maps for $U \rightarrow X$. Thus a reader not afraid of sites, will prefer to say that $\mathscr{F}$ is a sheaf on the big site of spaces over $\mathscr{M}$ (with the standard open topology).

2. A cartesian sheaf $\mathscr{F}$ is the same as a sheaf $\mathscr{F}_{X \rightarrow \mathscr{M}}=: \mathscr{F}_{X}$ on some atlas $X \rightarrow \mathscr{M}$ together with a descent datum, i.e., an isomorphism $\Phi: p r_{1}^{*} \mathscr{F}_{X} \rightarrow$ $p r_{2}^{*} \mathscr{F}_{X}$ on $X \times \mathscr{M} X$ which satisfies the cocycle condition on $X^{\times_{\mathscr{M}}^{3}}$ :

Given such a sheaf this defines a sheaf on every $T \rightarrow \mathscr{M}$, because we get an induced descent datum on $X \times \mathscr{M} T \rightarrow T$, this defines a sheaf on $T$. Of course, this is compatible with morphisms, since for $S \stackrel{f}{\longrightarrow} T \rightarrow \mathscr{M}$ the pull back commutes with descent.

Conversely, given a cartesian sheaf $\mathscr{F}$ and an atlas $X \rightarrow \mathscr{M}$ we get an isomorphism $\Phi:=\Phi_{p r_{2}}^{-1} \circ \Phi_{p r_{1}}: p r_{1}^{*} \mathscr{F}_{X} \rightarrow p r_{2}^{*} \mathscr{F}_{X}$ on $X \times \mathscr{M} X$. This satisfies the cocycle condition, since on $X^{\times_{\mathscr{M}}^{3}}$ we have $p r_{12}^{*}\left(\Phi_{p r_{1}}\right)=\Phi_{p r_{12}}^{-1} \circ \Phi_{p r_{1}}$ and therefore $p r_{12}^{*} \Phi=\Phi_{p r_{2}}^{-1} \circ \Phi_{p r_{1}}$. 
3. One might prefer to think only of cartesian sheaves on a stack, unfortunately this category does usually not contain enough injectives. But the subcategory of cartesian sheaves is a thick subcategory of all sheaves, i.e. a full category closed under kernels, quotients and extensions.

We can define global sections of a sheaf on $\mathscr{M}$. For cartesian sheaves we can simply choose an atlas $X \rightarrow \mathscr{M}$ and define

$$
\Gamma(\mathscr{M}, \mathscr{F}):=\operatorname{Ker}(\Gamma(X, \mathscr{F}) \longrightarrow \Gamma(X \times \mathscr{M} X)) .
$$

Lemma 4.3. For a cartesian sheaf $\mathscr{F}$ on $\mathscr{M}$ the group $\Gamma(\mathscr{M}, \mathscr{F})$ does not depend on the choice of the atlas.

Proof. First note that the lemma holds if $X$ is replaced by an open covering $X^{\prime}=\cup U_{i} \rightarrow X \rightarrow \mathscr{M}$, because $\mathscr{F}_{X \rightarrow \mathscr{M}}$ is a sheaf.

Secondly we only need to check the lemma for refinements, i.e. an atlas $X^{\prime} \rightarrow \mathscr{M}$ which factors $X^{\prime} \stackrel{f}{\longrightarrow} X \rightarrow \mathscr{M}$ such that $f$ has local sections. But then by assumption any global section defined via $X^{\prime}$ induces one on $X$.

Similarly to the above construction, one can - as for $G$-bundles - give a simplicial description of cartesian sheaves on a stack as follows: Choose an atlas $X \rightarrow \mathscr{M}$. Then a sheaf on $\mathscr{M}$ defines a sheaf on the simplicial space $X_{\bullet}$, i.e. a sheaf $\mathscr{F}_{n}$ on all $X_{n}$, together with isomorphisms for all simplicial maps $f:[m] \rightarrow[n]$ from $f^{*} \mathscr{F}_{n} \rightarrow \mathscr{F}_{m}$.

Again we call a sheaf on a simplicial space cartesian, is all $f^{*}$ are isomorphisms.

Conversely for any map $T \rightarrow \mathscr{M}$ a cartesian sheaf on $X_{\bullet}$ defines a sheaf on the covering $X \times \mathscr{M} T \rightarrow T$, via the formula 1 . This formula only defines global sections, but we can do the same for any open subset $U \subset T$.

Remark 4.4. Note that the functor $S h v(\mathscr{M}) \rightarrow S h v\left(X_{\bullet}\right)$ defined above is exact.

Example 4.5. A cartesian sheaf on a quotient stack $[X / G]$ is the same as a $G$-equivariant sheaf on $X$.

The category of sheaves of abelian groups on a stack $\mathscr{M}$ has enough injectives, so we want to define the cohomology of $H^{*}(\mathscr{M}, \mathscr{F})$ as the derived functor of the global section functor. By the last example, for quotients $[X / G]$ this will be the same as equivariant cohomology on $X$.

As noted before, to define cohomological functors we have to consider arbitrary sheaves on $\mathscr{M}$ resp. on $X_{\bullet}$. We define global sections as:

$$
\Gamma(\mathscr{M}, \mathscr{F}):=\lim _{\leftarrow} \Gamma\left(X, \mathscr{F}_{X \rightarrow \mathscr{M}}\right)
$$


Where the limit is taken over all atlases $X \rightarrow \mathscr{M}$, the transition functions for a commutative triangle $X^{\prime} \stackrel{f}{\rightarrow} X$ are given by the restriction maps $\Phi_{f, \varphi}$.

Lemma 4.6. For a cartesian sheaf $\mathscr{F}$ on a stack $\mathscr{M}$ the two notions of global sections coincide.

Proof. For any atlas $X \rightarrow \mathscr{M}$ the maps $X \times \mathscr{M} X \rightarrow \mathscr{M}$ are atlases as well. Thus we get a map

$$
\lim _{\leftarrow} \Gamma\left(X^{\prime}, \mathscr{F}_{X^{\prime} \rightarrow \mathscr{M}}\right) \rightarrow \operatorname{Ker}(\Gamma(X, \mathscr{F}) \Longrightarrow \Gamma(X \times \mathscr{M} X)) .
$$

Conversely we have seen in lemma 4.3 that we can define a map in the other direction as well. And it is not difficult to check that these are mutually inverse.

One tool to compute the cohomology of a sheaf on $\mathscr{M}$ is the spectral sequence given by the simplicial description above:

Proposition 4.7. Let $\mathscr{F}$ be a cartesian sheaf of abelian groups on a stack $\mathscr{M}$. Let $X \rightarrow \mathscr{M}$ be an atlas and $\mathscr{F} \bullet$ the induced sheaf on the simplicial space $X$. then there is an $E_{1}$ spectral sequence:

$$
E_{1}^{p, q}=H^{q}\left(X_{p}, \mathscr{F}_{p}\right) \Rightarrow H^{p+q}(\mathscr{M}, \mathscr{F}) .
$$

The spectral sequence is functorial with respect to morphisms $X \rightarrow Y$, for<smiles>[Y]=C[AlH]</smiles>
$\mathscr{M} \rightarrow \mathscr{N}$

atlases $X, Y$ of $\mathscr{M}$ and $\mathscr{N}$.

Proof. (e.g., [Del74],[Fri82]) For a cartesian sheaf $\mathscr{F}$ on $\mathscr{M}$ we denote by $\mathscr{F} \bullet$ the induced sheaf on $X_{\bullet}$. We first show that $H^{*}(\mathscr{M}, \mathscr{F})$ is the same as the cohomology of the simplicial space $X_{\bullet}$ with values in $\mathscr{F}_{\bullet}$.

Recall that global sections of a sheaf $\mathscr{F}_{\bullet}$ on $X_{\bullet}$ are defined as

$$
\Gamma\left(X_{\bullet}, \mathscr{F}_{\bullet}\right):=\operatorname{Ker}(\Gamma(X, \mathscr{F}) \Longrightarrow \Gamma(X \times \mathscr{M} X), \mathscr{F}) .
$$

Thus for any cartesian sheaf $\mathscr{F}$ on $\mathscr{M}$ we have $H^{0}\left(X_{\bullet}, \mathscr{F}_{\bullet}\right)=H^{0}(\mathscr{M}, \mathscr{F})$.

We can factor the the cohomology functor on $X_{\bullet}$ as follows: First $\mathbf{R} \pi_{\bullet}, *$ from the derived category of sheaves on $X_{\bullet}$ to the derived category of simplicial sheaves on $\mathscr{M}$, then the exact functor tot taking the total complex of a simplicial complex and finally take the cohomology over $\mathscr{M}$.

Now for any $U \rightarrow \mathscr{M}$ we can calculate $\left.\left(\mathbf{R} \pi_{\bullet, *} \mathscr{F}_{\bullet}\right)\right|_{U}$ as the direct image of the simplicial space $X_{\bullet} \times \mathscr{M} U \stackrel{\pi_{U}}{\longrightarrow} U$ over $U$. But for any sheaf $\mathscr{F}_{U}$ on $U$ we know 
that $\operatorname{tot}\left(\mathbf{R} \pi_{U, \bullet, *} \pi_{U}^{*} \mathscr{F}_{U}\right) \cong \mathscr{F}$, because $\pi_{U}$ has local sections: Indeed, since the claim is local on $U$ we may assume that $\pi_{U}$ has a section $s: U \rightarrow X \times \mathscr{M} U$. But if we denote $X_{U}:=X \times{ }_{\mathscr{M}} U$ then $X_{n} \times \mathscr{M} U=X_{U} \times_{U} \cdots \times_{U} X_{U}$ and therefore the section $s$ induces sections $X_{n} \rightarrow X_{n+1}$ which induce a homotopy on $\operatorname{tot}\left(\mathbf{R} \pi_{U, \bullet, *} \pi^{*} \mathscr{F}\right)$ proving that this complex is isomorphic to $\mathscr{F}$.

Thus we have shown that $H^{*}(\mathscr{M}, \mathscr{F})=H^{*}\left(X_{\bullet}, \mathscr{F}_{\bullet}\right)$.

The spectral sequence is defined via the same construction, factoring $H^{*}$ into $\mathbf{R} \Gamma \bullet(K):=\left(\mathbf{R} \Gamma\left(K_{n}\right)\right)_{n}$, which takes values in the derived category of simplicial complexes and the (exact) functor taking the associated total complex tot.

The spectral sequence is the spectral sequence of the double complex corresponding to the simplicial complex.

This spectral sequence gives one way to transport the properties of the cohomology of manifolds to stacks:

\section{Proposition 4.8.}

1. (Künneth Isomorphism) There is a natural isomorphism

$$
H^{*}(\mathscr{M} \times \mathscr{N}, \mathbb{Q}) \cong H^{*}(\mathscr{M}, \mathbb{Q}) \otimes H^{*}(\mathscr{N}, \mathbb{Q}) .
$$

2. (Gysin sequence) For smooth embeddings $\mathscr{Z} \hookrightarrow \mathscr{M}$ of codimension c there is a long exact sequence:

$$
\rightarrow H^{k-c}(\mathscr{Z}, \mathbb{Q}) \rightarrow H^{k}(\mathscr{M}, \mathbb{Q}) \rightarrow H^{k}(\mathscr{M}-\mathscr{Z}, \mathbb{Q}) \rightarrow
$$

In particular, the restriction $H^{k}(\mathscr{M}, \mathbb{Q}) \rightarrow H^{k}(\mathscr{M}-\mathscr{Z}, \mathbb{Q})$ is an isomorphism for $k<c-1$.

This helps to do some well known cohomology computations in the language of stacks:

Example 4.9. Let $G$ be a group acting trivially on a space $X$. To give a $G$-equivariant morphism from a $G$-bundle on a space $T$ to $X$ is the same as to give a map $T \rightarrow X$, thus $[X / G] \cong X \times[p t / G]$. And thus

$$
H^{*}([X / G], \mathbb{Q}) \cong H^{*}(X, \mathbb{Q}) \otimes H^{*}([p t / G], \mathbb{Q}) .
$$

Let $T \cong\left(S^{1}\right)^{n}$ be a torus. Then $\underline{B T} \cong\left(\underline{B S^{1}}\right)^{n}$, because any $T$-bundle is canonically the product of $S^{1}$-bundles, once an isomorphism $T \cong\left(S^{1}\right)^{n}$ is chosen. Thus $H^{*}(B T, \mathbb{Q}) \cong H^{*}\left(B S^{1}, \mathbb{Q}\right)^{\otimes n}$.

Finally we want to calculate $H^{*}\left(\left[p t / S^{1}\right], \mathbb{Q}\right) \cong \mathbb{Q}\left[c_{1}\right]$ a polynomial ring with one generator of degree 2. One way to do this is as follows: By the spectral sequence 4.7 we see that the morphisms $\left[\mathbb{C} / \mathbb{C}^{*}\right] \rightarrow\left[p t / \mathbb{C}^{*}\right] \leftarrow\left[p t / S^{1}\right]$ induce isomorphisms in cohomology, where the action of $\mathbb{C}^{*}$ on $\mathbb{C}$ is the standard action. This is because $H^{*}\left(\mathbb{C} \times\left(\mathbb{C}^{*}\right)^{n}, \mathbb{Q}\right) \cong H^{*}\left(\left(S^{1}\right)^{n}, \mathbb{Q}\right)$. The same is true 
for $\left[\mathbb{C}^{N} / \mathbb{C}^{*}\right] \rightarrow[p t / \mathbb{C}]$. But here we can use the Gysin sequence: The inclusion $0 \rightarrow \mathbb{C}^{N}$ induces a closed embedding $\left[p t / \mathbb{C}^{*}\right] \rightarrow\left[\mathbb{C}^{N} / \mathbb{C}^{*}\right]$ of codimension $N$. The open complement $\left[\mathbb{C}^{N}-0 / \mathbb{C}^{*}\right] \cong \mathbb{C P}^{N-1}$, because the $\mathbb{C}^{*}$ action is free outside the origin. This proves the claim.

For the definition of $f$ ! maps in $K$-theory we need to define normal bundles, at least for nice representable morphisms:

Lemma/Definition 4.10. Let $f: \mathscr{M} \rightarrow \mathscr{N}$ be a representable morphism of differentiable stacks satisfying one of the following conditions:

1. $f$ is a smooth submersion.

2. $f$ is a smooth embedding.

Let $Y \stackrel{p}{\longrightarrow} \mathscr{N}$ be any smooth atlas of $\mathscr{N}$. Then normal bundle $T_{\mathscr{M} \times \mathscr{N}} Y \rightarrow Y$ descends to a vector bundle $T_{\mathscr{M} \rightarrow \mathscr{N}}$ on $\mathscr{M}$. This does not depend on the choice of $Y$ and is called the normal bundle to $f$.

Proof. We only need to note that formation of the normal bundle commutes with pull-back. Therefore the two pull backs of the normal-bundle of $\mathscr{M} \times \mathscr{N} \rightarrow$ $Y$ to $\left(\mathscr{M} \times \mathscr{N}_{Y} Y\right) \times \mathscr{M}(\mathscr{M} \times \mathscr{N} Y)$ are both canonically isomorphic to the normal bundle to $\left(\mathscr{M} \times \mathscr{N}\left(Y \times_{\mathscr{N}} Y\right) \rightarrow\left(Y \times_{\mathscr{N}} Y\right)\right.$. Therefore the bundle descends to a bundle on $\mathscr{M}$.

Since for manifolds formation of the normal bundle commutes with pullbacks, the same holds for stacks:

Corollary 4.11. If $\mathscr{M} \rightarrow \mathscr{N}$ is a morphism as in the above lemma and $g: \mathscr{N}^{\prime} \rightarrow \mathscr{N}$ is an arbitrary morphism, then $T_{\mathscr{M} \times \mathscr{N}^{\prime} \mathfrak{N}^{\prime} \rightarrow \mathscr{N}^{\prime}} \cong g^{*} T_{\mathscr{M} \rightarrow \mathscr{N}}$.

Similarly one gets short exact sequences for the normal bundle of a composition, because the corresponding sequences for an atlas descend.

Tangent spaces to differentiable stacks will only be stack-versions of vector bundles. Nevertheless define:

Lemma/Definition 4.12 (Tangent stacks). Let $\mathscr{M}$ be a differentiable stack and $X \rightarrow \mathscr{M}$ be a smooth atlas. Then we can take the tangent spaces to the groupoid $X_{\bullet}$ :

$$
T(X \times \mathscr{M} X \times \mathscr{M} X) \Longrightarrow T\left(X_{\times} \mathscr{M} X\right) \Longrightarrow T X
$$

by functoriality this is again a groupoid, the quotient $[T X / T(X \times \mathscr{M} X)]$ is independent of the choice of $X$ and is called $T \mathscr{M}$, the tangent stack to $\mathscr{M}$. 
The fibers of the projection $T \mathscr{M} \rightarrow \mathscr{M}$ are isomorphic to $[V / W]$, where $V, W$ are finite dimensional vector spaces, and $W$ acts on $V$ by some linear map $W \rightarrow V$, which is not injective in general.

\section{5. $S^{1}$-Gerbes or twists}

Informally a gerbe ${ }^{(2)}$ over some space $X$ is a stack $\mathscr{X} \rightarrow X$ which has the same points as $X$, i.e. the points of $X$ are isomorphism classes of objects in $\mathscr{X}(p t)$. An $S^{1}$-gerbe is a gerbe such that the automorphism groups of all points $p t \rightarrow \mathscr{X}$ are isomorphic to $S^{1}$ in a continuous way.

The easiest example of such an object is $\left[p t / S^{1}\right] \rightarrow p t$. More generally these objects occur naturally in many moduli-problems, e.g. every $U(n)$-bundle with flat connection on a compact Riemann surface has an automorphism group $S^{1}$, in good situations the stack of such objects is a $S^{1}$-gerbe over the coarse moduli space. This gerbe gives the obstruction to the existence of a Poincaré bundle on the coarse moduli-space. Finally these objects seem to appear naturally in $K$-theoretic constructions, since the choices of Ppin $^{c}$-structures on an oriented bundle form a $S^{1}$-gerbe (locally there is only one such choice, but the trivial Spin $^{c}$-bundle has more automorphisms).

Definition 5.1. Let $X$ be a space. A stack $\mathscr{X} \stackrel{\pi}{\longrightarrow} X$ is called a gerbe over $X$ if

1. $\pi$ has local sections, i.e., there is an open covering $\cup U_{i}=X$ and sections $s_{i}: U_{i} \rightarrow \mathscr{X}$ of $\left.\pi\right|_{U_{i}}$.

2. Locally over $X$ all objects of $\mathscr{X}$ are isomorphic, i.e., for any two objects $t_{1}, t_{2} \in \mathscr{X}(T)$ there is a covering $\cup U_{i}=T$ such that $\left.\left.t_{1}\right|_{U_{i}} \cong t_{2}\right|_{U_{i}}$.

A gerbe $\mathscr{X} \rightarrow X$ is called a (continuous) $S^{1}$-gerbe if for any $T \rightarrow X$, together with a section $s: T \rightarrow \mathscr{X}$ there is an isomorphism $A u t(s):=(T \times \mathscr{X} T) \times_{T \times T}$ $T \cong S^{1} \times T$ as family of groups over $T$, which is compatible with composition of morphisms $T^{\prime} \stackrel{s^{\prime}}{\longrightarrow} T \stackrel{s}{\longrightarrow} \mathscr{X}$.

\section{Remarks 5.2.}

1. As one might expect, the condition that the automorphism group of any object is $S^{1}$ implies that for any section $s: T \rightarrow \mathscr{X}$ the map $T \times{ }_{X} T \rightarrow T \times{ }_{X} T$ is an $S^{1}$-bundle. Since the fibres of this map are given by two points together with a morphism between the images in $\mathscr{X}$ the fibres are $S^{1}$-torsors. To see that the map is indeed a locally trivial bundle one can replace $T$ by $T \times \mathscr{X} T$

\footnotetext{
${ }^{(2)}$ Gerbe is the french word for sheaf, to avoid another wrong translation (cf. faisceaux, champ etc.) there seems to be an agreement to keep the french word - or at least its spelling.
} 
in the above definition to get an isomorphism $\left(T \times X_{\mathscr{X}} T\right) \times_{\left(T \times{ }_{X} T\right)}\left(T \times \times_{\mathscr{X}} T\right) \cong$ $S^{1} \times(T \times \mathscr{X} T)$ (one only has to write down, the functor represented by the left hand side).

2. An $S^{1}$-gerbe over a topological/differentiable space $X$ is always a topological/differentiable stack, an atlas is given by the sections $s_{i}$. By the previous remark we know that $U_{i} \times \mathscr{X} U_{i}$ is a space and the two projections are $S^{1}$ bundles, in particular smooth.

This also shows that we might replace the condition that the automorphism groups are isomorphic to $S^{1}$ for all objects, by the same condition for sections of one atlas of $X$. Representability of arbitrary fibered products $T_{1} \times \mathscr{X} T_{2}$ then follows, since locally over $X$ we can glue $S^{1}$-bundles. (This definition will be explained more carefully below.)

3. As in the case of bundles, one there is also a notion of discrete $S^{1}$-gerbe, simply by choosing the discrete topology for $S^{1}$ in the above definition.

4. Any $S^{1}$-gerbe on a contractible space is trivial, i.e. isomorphic to

$$
X \times\left[p t / S^{1}\right] \rightarrow X .
$$

Perhaps this is obvious. If not, one might reason as follows: Choose a covering $U_{i}$ of $X$ with sections $s_{i}: U_{i} \rightarrow \mathrm{X}$, such that all $U_{i}, U_{i} \cap U_{j}$ are contractible. Then $U_{i} \times \mathscr{X} U_{j} \rightarrow U_{i} \cap U_{j}$ is a locally trivial $S^{1}$ bundle, thus trivial. Therefore the obstruction to glue the sections $s_{i}$ gives an element in $H^{2}\left(X, S^{1}\right)=0$ (the classification of gerbes will show that this $H^{2}$ classifies $S^{1}$-gerbes).

5. A gerbe with a section is called neutral. Gerbes which are isomorphic to $X \times[p t / G] \rightarrow X$ for some group $G$ are called trivial gerbes over $X$.

We will need a generalization of the above, to include gerbes over topological stacks $\mathscr{M}$ instead of spaces $X$. Again we only have to replace coverings by representable morphisms with local sections:

Definition 5.3. Let $\mathscr{M}$ be a topological stack. A stack $\mathscr{M}^{\tau} \stackrel{\pi}{\longrightarrow} \mathscr{M}$ is called a gerbe over $\mathscr{M}$ if

1. $\pi$ has local sections, i.e. there is an atlas $X \rightarrow \mathscr{M}$ and a section $s: X \rightarrow$ $\mathscr{M}^{\tau}$ of $\left.\pi\right|_{X}$.

2. Locally over $\mathscr{M}$ all objects of $\mathscr{M}^{\tau}$ are isomorphic, i.e. for any two objects $t_{1}, t_{2} \in \mathscr{M}(T)$ and lifts $s_{1}, s_{2} \in \mathscr{M}^{\tau}(T)$ with $\pi\left(s_{i}\right) \cong t_{i}$, there is a covering $\cup U_{i}=T$ such that $\left.\left.s_{1}\right|_{U_{i}} \cong s_{2}\right|_{U_{i}}$.

A gerbe $\mathscr{M}^{\tau} \rightarrow \mathscr{M}$ is called a (continuous) $S^{1}$-gerbe if there is an atlas $X \stackrel{p}{\longrightarrow}$ $\mathscr{M}$ of $\mathscr{M}$, a section $\left(s: X \rightarrow \mathscr{M}^{\tau}, \varphi: \pi \circ s \Rightarrow p\right)$ such that there is an isomorphism $\Phi: \operatorname{Aut}(s / p):=\left(X \times \mathscr{M}^{\tau} X\right) \times_{X \times \mathscr{M} X} X \cong S^{1} \times X$ as family of 
groups over $X$, such that on $X \times \mathscr{M} X$ the diagram

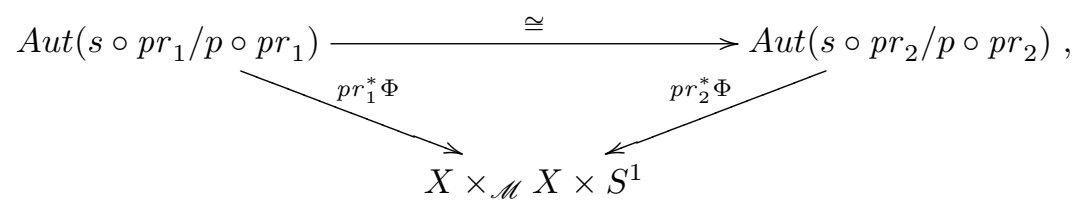

where the horizontal map is the isomorphism given by the universal property of the fibered product, commutes (i.e. the automorphism groups of objects of $\tilde{\mathscr{M}}$ are central extensions of those of $\mathscr{M}$ by $S^{1}$ ).

\section{Example 5.4.}

1. The easiest example of a $S^{1}$-gerbe on a quotient stack $[X / G]$ is given by a central extension $S^{1} \rightarrow \tilde{G} \stackrel{p r}{\longrightarrow} G$, then $\tilde{G}$ also acts on $X$ and $p r$ induces a map $[X / \tilde{G}] \stackrel{\pi}{\longrightarrow}[X / G]$, which defines a gerbe over $[X / G]$ : The atlas $X \rightarrow[X / G]$ lifts to $[X / \tilde{G}]$, this shows (1). And (2) follows, because locally any map $T \rightarrow G$ can be lifted to $\tilde{G}$.

Finally the map $S^{1} \rightarrow \tilde{G}$ induces a morphism $\left[X / S^{1}\right] \rightarrow[X / \tilde{G}]$ which induces an isomorphism $X \times\left[p t / S^{1}\right] \cong\left[X / S^{1}\right] \stackrel{\cong}{\longrightarrow} X \times_{[X / G]}[X / \tilde{G}]$. This shows the last condition of the definition.

2. This generalizes to groupoids: An extensions of a groupoid $\Gamma_{1} \longrightarrow \Gamma_{0}$ by $S^{1}$ is a groupoid $\tilde{\Gamma}_{1} \longrightarrow \Gamma_{0}$ with a morphism: $\tilde{\Gamma}_{1} \Longrightarrow \Gamma_{0}$ such that

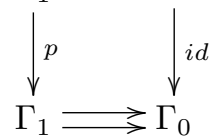

$p$ is an $S^{1}$-bundle and the $S^{1}$-action commutes with the source and target morphisms.

As before this defines a $S^{1}$-gerbe $\left[\Gamma_{0} / \tilde{\Gamma}_{1}\right] \rightarrow\left[\Gamma_{0} / \Gamma_{1}\right]$.

\section{Remarks 5.5.}

1. As before a $S^{1}$-gerbe is always a differentiable stack, the section $s: X \rightarrow$ $\mathscr{M}^{\tau}$ of the particular atlas $X \rightarrow \mathscr{M}$ is an atlas for $\mathscr{M}^{\tau}$ :

The map $s$ is representable, because by base-change (Lemma 2.6) $X \times \mathscr{M}$ $\mathscr{M}^{\tau} \rightarrow \mathscr{M}^{\tau}$ is representable and the canonical map $X \rightarrow X \times \mathscr{M} \mathscr{M}^{\tau}$ induced by $s$ is surjective by definition and representable since $X \times_{X \times \mathscr{M} \mathscr{M}^{\tau}} X \cong$ $\operatorname{Aut}(s / p) \cong S^{1} \times X$.

Thus the free action of $A u t(s / p)$ induces a structure of an $S^{1}$ bundle on $X \times \mathscr{M}^{\tau} X \rightarrow X \times \mathscr{M} X$. As in remark 5.2(1) one can prove that this map is a locally trivial $S^{1}$-bundle. 
Furthermore, the last condition of the definition ensures, that this defines an $S^{1}$-extension of groupoids. Thus every $S^{1}$ gerbe can be constructed as in the example given above.

2. Since we just saw that for any $T \stackrel{s}{\longrightarrow} \mathscr{M}^{\tau} \stackrel{\pi}{\longrightarrow} \mathscr{M}$ the group Aut $(s / s \circ$ $\pi)$ is representable, locally canonically isomorphic to $S^{1}$ we get a canonical isomorphism $\operatorname{Aut}(s / s \circ \pi) \cong S^{1} \times T$. Thus again we could have used this as a definition of $S^{1}$-gerbes.

3. Thus we can pull-back gerbes: For any $\mathscr{N} \rightarrow \mathscr{M}$ and any $S^{1}$-gerbe $\mathscr{M}^{\tau} \rightarrow \mathscr{M}$ the stack $\mathscr{N}^{\tau}:=\mathscr{M}^{\tau} \times \mathscr{M}_{\mathscr{N}}$ is a $S^{1}$-gerbe over $\mathscr{N}$, since for any $T \rightarrow \mathscr{N}^{\tau}$ we have $T \times \mathscr{N} \mathscr{N}^{\tau}=T \times \mathscr{N}\left(\mathscr{N} \times \mathscr{M} \mathscr{M}^{\tau}\right) T=T \times \mathscr{M}^{\tau}$.

4. A morphism of $S^{1}$-gerbes is a morphism of the corresponding stacks overthe base stack, which induces the identity on the central $S^{1}$ automorphisms of the objects.

As before we call a gerbe neutral if it has a section. To state this in a different way recall that for any bundle $\mathscr{P}$ on $\tilde{\mathscr{M}}$ and any $s: T \rightarrow \tilde{\mathscr{M}}$ we get an action of $A u t(s)$ on $s^{*} \mathscr{P}$. In particular for a line bundle $\mathscr{L}$ the pull back carries an $S^{1}$-action. Thus $S^{1}$ acts on every fibre by a character $\chi=()^{n}: S^{1} \rightarrow S^{1}$, where $n$ is some integer, constant on connected components of $T$ resp. $\tilde{\mathscr{M}}$. A line bundle on $\tilde{\mathscr{M}}$ is called of weight $n$ if $n$ is constant on all connected components.

Lemma 5.6. For a $S^{1}$-gerbe $\pi: \tilde{\mathscr{M}} \rightarrow \mathscr{M}$ the following are equivalent:

1. $\tilde{\mathscr{M}} \rightarrow \mathscr{M}$ has a section $s$.

2. $\tilde{\mathscr{M}} \cong\left[p t / S^{1}\right] \times \mathscr{M}$ as stacks over $\mathscr{M}$.

3. There is a unitary line bundle of weight 1 on $\tilde{\mathscr{M}}$.

Proof: Of course 2. $\Rightarrow 1$.. Furthermore, the universal bundle $p t \rightarrow\left[p t / S^{1}\right]$ is of weight 1 , thus 2 . $\Rightarrow 3$.

Given a unitary line bundle of weight 1 we get a morphism $\tilde{\mathscr{M}} \rightarrow \mathscr{M} \times B S^{1}$. This map induces an isomorphism on automorphism groups of objects, because the kernel of the map $A u t_{\tilde{\mathscr{M}}} \rightarrow A u t_{\mathscr{M}}$ is $S^{1}$ and this kernel is mapped isomorphically to the automorphisms of $S^{1}$ bundles, since we started from a bundle of weight 1 . The map is also locally essentially surjective on objects, because locally every object of $\mathscr{M}$ can be lifted to an object of $\tilde{\mathscr{M}}$ and locally every $S^{1}$-bundle is trivial. And finally the map is a gerbe, since locally all objects in the fibre are isomorphic. This implies that the map is an isomorphism.

This also shows, that the total space of the $S^{1}$-bundle is isomorphic to $\mathscr{M}$, thus any line bundle of weight 1 induces a section. 
Finally, given a section $\mathscr{M} \rightarrow \tilde{\mathscr{M}}$ we get an isomorphism $S^{1} \times \mathscr{M} \cong \mathscr{M} \times \tilde{\mathscr{M}}$ $\mathscr{M} \times \mathscr{M} \times \mathscr{M} \mathscr{M}=\mathscr{M} \times \tilde{\mathscr{M}} \mathscr{M}$. The compatibility condition shows, that this makes $\mathscr{M}$ into an $S^{1}$-bundle over $\mathscr{M}$.

Remark 5.7. The descriptions 2. and 3. of the lemma show that line bundles on $\mathscr{M}$ act on trivializations of a $S^{1}$-gerbe. In description 2 . this is because a morphism to $\left[p t / S^{1}\right]$ is the same as a unitary line bundle on $\mathscr{M}$ and in description 3 . one sees, that two line bundles of weight 1 differ by a line bundle on $\mathscr{M}$.

There is a description of isomorphism classes of gerbes in terms of cocycles, see for example [Bre94] and [Cra]. We write $\mathscr{S}^{1}$ for the sheaf of continuous sections of the trivial bundle $S^{1} \times \mathscr{M} \rightarrow \mathscr{M}$ :

\section{Proposition 5.8.}

1. Let $\mathscr{M}$ be a topological stack. Then there is a natural bijection

$\left\{\right.$ Isom. classes of $S^{1}$-gerbes over $\left.\mathscr{M}\right\} \cong H^{2}\left(\mathscr{M}, \mathscr{S}^{1}\right)$.

The same holds if $S^{1}$ is replaced by any abelian, topological group.

2. If $\mathscr{M}$ is a differentiable stack such that the diagonal $\Delta: \mathscr{M} \rightarrow \mathscr{M} \times$ $\mathscr{M}$ is proper, then the boundary map of the exponential sequence induces an isomorphism $H^{2}\left(\mathscr{M}, \mathscr{S}^{1}\right) \stackrel{\cong}{\longrightarrow} H^{3}(\mathscr{M}, \mathbb{Z})$.

Indication of the proof: The two parts of the theorem are of very different nature, they are only put in one statement, because the cocycles in (2), called Dixmier-Douady classes, are often used to characterize gerbes.

For the first part we will first describe how to associate a cohomology class to a gerbe $\mathscr{M}^{\tau}$.

Choose an atlas $X \rightarrow \mathscr{M}$ which is the disjoint union of contractible spaces, e. g., take any atlas $Y$ and then chose a covering of $Y$ by contractible spaces. We use the spectral sequence $H^{p}\left(X^{\times_{\mathscr{M}}^{q+1}}, \mathscr{S}^{1}\right) \Rightarrow H^{p+q}\left(\mathscr{M}, \mathscr{S}^{1}\right)$ to calculate $H^{2}\left(\mathscr{M}, \mathscr{S}^{1}\right)$. By the choice of $X$ this is:

$$
\begin{aligned}
& H^{2}\left(X, \mathscr{S}^{1}\right)=0 \\
& H^{1}\left(X, \mathscr{S}^{1}\right)=0 \\
& H^{0}\left(X, \mathscr{S}^{1}\right) \longrightarrow H^{1}\left(X \times \mathscr{M} X, \mathscr{S}^{1}\right) \stackrel{d^{1}}{\longrightarrow} H^{1}\left(\times^{3} \mathscr{M}, \mathscr{S}^{1}\right) \\
& \longrightarrow H^{0}\left(X \times \mathscr{M} X, \mathscr{S}^{1}\right) \stackrel{d^{1}}{\longrightarrow} H^{0}\left(X^{\times} \mathscr{M}, \mathscr{S}^{1}\right) \stackrel{d^{1}}{\longrightarrow} H^{0}\left(X^{\times} \mathscr{M}, \mathscr{S}^{1}\right)
\end{aligned}
$$


Where the differentials $d^{1}$ are given by the alternating sum over the pullbacks (since the spectral sequence is constructed from a simplicial object by taking alternating sums of the simplicial maps).

As explained before the choice of a trivialization of the pull-back $X \stackrel{s}{\longrightarrow}$ $X^{\tau}=X \times \mathscr{M} \mathscr{M}^{\tau}$ of $\mathscr{M}^{\tau}$ to $X$ induces a map $\tilde{p}: X \rightarrow \mathscr{M}^{\tau}$ and an $S^{1}$-bundle

$$
P:=X \times \mathscr{M}^{\tau} X=I \operatorname{som}\left(\tilde{p} \circ p_{1}, \tilde{p} \circ p_{2}\right) \rightarrow \operatorname{Isom}\left(p \circ p_{1}, p \circ p_{2}\right)=X \times \mathscr{M} X
$$

thus a class in $H^{1}\left(X \times \mathscr{M} X, \mathscr{S}^{1}\right)$.

This actually lies in the kernel of $d^{1}$, because on $X^{\times_{\mathscr{M}}^{3}}$ the composition induces an isomorphism

$$
\Phi_{123}: \operatorname{Isom}\left(\tilde{p} \circ p_{1}, \tilde{p} \circ p_{2}\right) \otimes I \operatorname{som}\left(\tilde{p} \circ p_{2}, \tilde{p} \circ p_{3}\right) \stackrel{\cong}{\longrightarrow} \operatorname{Isom}\left(\tilde{p} \circ p_{1}, \tilde{p} \circ p_{3}\right) .
$$

We will see below, that the associativity of the composition exactly means that this also lies in the kernel of $d^{2}$. Furthermore we may view $\Phi_{123}$ as a section of the bundle $p_{12}^{*} P \otimes p_{23}^{*} P \otimes\left(p_{13}^{*} P\right)^{-1}$. This shows that the choices of $\Phi_{123}$, which define an associative composition form a torsor for $\operatorname{ker}\left(H^{0}\left(X^{\times^{3} \mathscr{M}}, \mathscr{S}^{1}\right) \rightarrow\right.$ $\left.H^{0}\left(X^{\times^{4}}, \mathscr{S}^{1}\right)\right)$. Two such choices define isomorphic gerbes, whenever we change $\Phi_{123}$ by an automorphism of $P$, i.e., an element of $H^{0}\left(X^{\times_{\mathscr{M}}^{2}}, \mathscr{S}^{1}\right)$.

To see that this construction defines an element in $H^{2}\left(\mathscr{M}, \mathscr{S}^{1}\right)$ we have to check that the we found an element in the correct extension of the $E_{2}^{11}$ by the $E_{2}^{0,2}$ term and that the differential $d^{2}$ corresponds to associativity. Accepting this for a moment, we see that the process can be reversed:

Cohomology classes as above can be used to glue a groupoid over $X \times \mathscr{M} X \rightarrow$ $X$. The boundary maps in the spectral sequence assure the associativity of the composition. (One should note that in the construction $\Phi_{123}$ also defines isomorphisms $P^{-1} \cong t w^{*} P$ where $t w=()^{-1}: X \times \mathscr{M} X \rightarrow X \times \mathscr{M} X$ is the inverse map of the groupoid $X^{\times} \mathscr{M}$, and trivialization of the restriction of $P$ to the diagonal $\left.P\right|_{\Delta(X)}$.)

To analyze the differentials of the spectral sequence we have to recall its construction: We have to chose acyclic resolutions of $S^{1}$ on $X^{\times_{\mathscr{M}}^{i}}$. Thus we choose a covering $X_{\alpha}^{2}$ of $X \times \mathscr{M} X$ such that all the intersections $X_{\alpha_{1}}^{2} \cap \cdots \cap X_{\alpha_{3}}^{2}$ are acyclic (this condition could be avoided if we would allow for another index). Then we chose a covering $X_{\beta}^{3}$ of $X^{\times_{\mathscr{M}}^{3}}$ which has the same property, such that all projections $p r_{i j}: X^{\times_{\mathscr{M}}^{3}} \rightarrow X^{\times_{\mathscr{M}}^{2}}$ map $X_{\beta}^{3}$ to some $X_{p r_{i j}(\beta)}^{2}$. We do the same for $X^{\times^{4}}$ and get a covering $X_{\gamma}^{4}$. Taking global sections of $S^{1}$ over these spaces we get a double complex from which the spectral sequence is induced, the total complex calculates $H^{*}\left(\mathscr{M}, \mathscr{S}^{1}\right)$. Thus writing $X_{\alpha \alpha^{\prime}}^{2}$ for the intersection 
$X_{\alpha}^{2} \cap X_{\alpha^{\prime}}^{2}$ we calculate $H^{2}\left(\mathscr{M}, \mathscr{S}^{1}\right)$ as the cohomology of:

$$
\begin{aligned}
\bigoplus_{\alpha} H^{0}\left(X_{\alpha}^{2}\right) & \stackrel{d_{1}}{\longrightarrow} \bigoplus_{\alpha, \alpha^{\prime}} H^{0}\left(X_{\alpha \alpha^{\prime}}^{2}, \mathscr{S}^{1}\right) \oplus \bigoplus_{\beta} H^{0}\left(X_{\beta}^{3}, \mathscr{S}^{1}\right) \\
& \left.\stackrel{d_{2}}{\longrightarrow} \bigoplus_{\alpha \alpha^{\prime} \alpha^{\prime \prime}} H^{0}\left(X_{\alpha \alpha^{\prime} \alpha^{\prime \prime}}^{2}, \mathscr{S}^{1}\right) \oplus \bigoplus_{\beta, \beta^{\prime}} H^{0}\left(X_{\beta \beta^{\prime}}^{3}, \mathscr{S}^{1}\right) \oplus \bigoplus_{\gamma} H^{(} X_{\gamma}^{4}, \mathscr{S}^{1}\right)
\end{aligned}
$$

And the differentials are the sum of the simplicial and the covering differentials. Thus the components of $d_{2}$ are:

$$
\begin{aligned}
d_{2}\left(s_{\alpha \alpha^{\prime}}, s_{\beta}\right)_{\alpha, \alpha^{\prime}, \alpha^{\prime \prime}} & =s_{\alpha \alpha^{\prime}} s_{\alpha \alpha^{\prime \prime}}^{-1} s_{\alpha^{\prime} \alpha^{\prime \prime}} \\
d_{2}\left(s_{\alpha \alpha^{\prime}}, s_{\beta}\right)_{\beta, \beta^{\prime}} & =p r_{12}^{*} s_{p r_{12}(\beta) p r_{12}\left(\beta^{\prime}\right)} p r_{13}^{*} s_{p r_{13}(\beta) p r_{13}\left(\beta^{\prime}\right)}^{-1} r_{23}^{*} s_{p r_{23}(\beta) p r_{23}\left(\beta^{\prime}\right)}-s_{\beta}+s_{\beta^{\prime}} \\
d_{2}\left(s_{\alpha \alpha^{\prime}}, s_{\beta}\right)_{\gamma} & =p r_{123}^{*} s_{p r_{123}(\gamma)} r_{124}^{*} s_{p r_{124}(\gamma)}^{-1} p r_{134}^{*} s_{p r_{134}(\gamma)} p r_{234}^{*} s_{p r_{234}(\gamma)}^{-1}
\end{aligned}
$$

More precisely, the indices on the right hand side depend on the projections. If the first component is zero $s_{\alpha \alpha^{\prime}}$ defines an $S^{1}$-bundle $P$ on $X \times \mathscr{M} X$. The vanishing of the second summand assures that $s_{\beta}$ defines a section of $p r_{12}^{*} P \otimes$ $p r_{13} * P^{-1} \otimes p r_{23}^{*} P$. And finally the third summand guarantees associativity as claimed.

The second part of the proposition depends on the existence of a Haarmeasure on compact groupoids (i.e. groupoids defining stacks with proper diagonal $\mathscr{M} \rightarrow \mathscr{M} \times \mathscr{M}$, in particular all automorphism groups of objects are proper over the parameter space).

Using this Crainic [Cra] shows that a generalization of the Poincaré lemma holds for such stacks, i.e. the sheaves of continuous $\mathbf{R}$-valued functions are acyclic. Therefore by the exponential sequence $H^{2}\left(\mathscr{M}, \mathscr{S}^{1}\right) \cong H^{3}(\mathscr{M}, \mathbb{Z})$.

Remark 5.9. As one might expect from the proof above, the group structure of $H^{2}\left(X, \mathscr{S}^{1}\right)$ can also be implemented as an operation on stacks: Given $S^{1}$-gerbes $\mathscr{M}^{\tau}, \mathscr{M}^{\tau^{\prime}}$ on $\mathscr{M}$ one can take the fibred product $\mathscr{M}^{\tau} \times \mathscr{M}^{\mathscr{M}^{\tau^{\prime}}}$, which is an $S^{1} \times S^{1}$ and forget the anti-diagonal $S^{1}$-automorphisms. To avoid technical arguments we can simply choose an atlas $X \rightarrow \mathscr{M}$ on which both gerbes are trivial. Then we have already seen that $X \times \mathscr{M}^{\tau} \times \mathscr{M}_{\mathscr{M}^{\tau}} X \rightarrow X \times \mathscr{M} X$ is an $S^{1} \times S^{1}$-bundle and the multiplication $S^{1} \times S^{1} \rightarrow S^{1}$ defines an associated $S^{1}$-bundle $X_{1} \rightarrow X \times \mathscr{M} X$ and it is not difficult to check, that this defines a groupoid $X_{1} \gg X$.

In the special case of quotient stacks and gerbes given by two group extensions this is simply the Yoneda product of extensions. 
Another description of gerbes is via projective bundles. Given any (possibly finite dimensional) Hilbert space $H$. One gets an exact sequence of groups:

$$
1 \rightarrow S^{1} \rightarrow \mathrm{U}(H) \rightarrow \mathrm{PU}(H) \rightarrow 1
$$

By the first example of gerbes this defines an $S^{1}$-gerbe $B U \rightarrow B$ PU. In particular for any PU bundle $P$ on a space $X$ we can pull back this gerbe to $X$ via the classifying morphism $X \rightarrow B \mathrm{PU}$. The category of sections $X \times_{B \mathrm{PU}} B \mathrm{U}(T)$ is the category of $U$ bundles on $T$ together with an isomorphism of the associated PU bundle and the pull back of $P$ to $T$.

This shows that the gerbe obtained in this way corresponds to the image of $P$ under the boundary map $\delta: H^{1}(X, \mathrm{PU}) \rightarrow H^{2}\left(X, \mathscr{S}^{1}\right)$. In particular if $H$ is $n$-dimensional we may factorize this map via the sequence:

$$
0 \rightarrow \mathbb{Z} / n \mathbb{Z} \rightarrow \mathrm{SU}(n) \rightarrow \mathrm{PU}(n) \rightarrow 0
$$

i.e., the classes obtained in this way are $n$-torsion.

For the purpose of this Seminar it will be sufficient to note that the gerbes that arise naturally in $K$-theory are always obtained by PU bundles, this will be explained in the next section.

If $X$ is a manifold (and not a stack), then the fact that $B \mathrm{PU}$ is a $K(\mathbb{Z}, 3)$ space (if $H$ is an infinite dimensional Hilbert space) shows, that $\delta$ is an isomorphism, thus any $S^{1}$ gerbe arises in this way.

This is less clear for differentiable stacks, and Proposition 2.38 in [LTX] gives the result. Unfortunately, since I am not an analyst, their proof is to short for me. In section 6 we will prove that all $S^{1}$-gerbes arise from projective bundles, if the stack is a local quotient stack, a notion also defined in that section.

In $K$-theory one can define Thom-isomorphisms for Spin ${ }^{c}$-bundles and one can do the same for bundles on stacks (although one has to be a bit careful with the definition the Thom-space of a bundle). As remarked before the choices of $S p i n^{c}$-structure define a $S^{1}$ gerbe, simply pulling back the universal gerbe $B \operatorname{Spin}^{c} \rightarrow B S U(n)$. Thus every bundle $P$ on a a space $X$ defines a gerbe $\overline{X^{\tau} \rightarrow X}$ such that the pull back of $P$ to $X^{\tau}$ has a canonical Spin ${ }^{c}$ structure. (We get a stack and not a space, because the sequence of groups is $S^{1} \rightarrow$ Spin $^{c} \rightarrow$ SO in contrast to orientation problems where the cokernel imposes the obstruction).

If the bundle is not orientable one first has to chose some $\mathbb{Z} / 2$ covering on which one chooses an orientation. And then one takes the above gerbe on the orientation covering. 
Again one has to be careful defining a group structure on these objects, since if we have two bundles which admit Spin $^{c}$-structures on the orientation cover, their tensor product does not necessarily admit a $\operatorname{Spin}^{c}$ structure on the sum of the orientation coverings.

The obstruction comes from the universal example on $B \mathbb{Z} / 2 \times B \mathbb{Z} / 2$ and this gives a geometric description of the cup product of two torsion-classes:

Lemma 5.10. Given finite abelian groups $A, B, C$ and a bilinear form $<$ , >: $A \times B \rightarrow C$, then:

1. $<,>$ defines an abelian extension $0 \rightarrow C \rightarrow G \rightarrow A \times B \rightarrow 0$ by the cocycle $\sigma\left(a, b, a^{\prime}, b^{\prime}\right)=<a,-b^{\prime}>+<a^{\prime},-b>$.

2. Given an $A$-bundle $P_{A}$ and $a B$-bundle $P_{B}$ on a space $X$ corresponding to classes $c\left(P_{A}\right) \in H^{1}(X, A), c\left(P_{B}\right) \in H^{1}(X, B)$. Define a $C$ gerbe on $X$, given by the pull back of the gerbe $B G \rightarrow B A \times B B$ defined in (1), via the classifying map $X \rightarrow B A \times B B$. The Dixmier Douady class of this gerbe is the cup product $c\left(P_{A}\right) \cup c\left(P_{B}\right)$.

Proof. Since the cup product commutes with pull-backs, we only may assume $X=B A \times B B$ and take $P_{A}, P_{B}$ the universal bundles.

In this case the standard atlas $p t \rightarrow B A \times B B$ is acyclic, as well as all fibered products $p t \times_{B(A \times B)} \cdots \times \times_{B(A \times B)} p t$.

Thus the spectral sequence we used to calculate the Dixmier-Douady classes is a complex. The class of the universal $C$-gerbe therefore is given by the cocycle $s\left(a, b, a^{\prime}, b^{\prime}\right)=\left\langle a,-b^{\prime}\right\rangle+\left\langle a^{\prime},-b\right\rangle$. And the same cocycle represents the cup-product.

\section{Local quotient stacks}

Freed, Hopkins and Teleman define $K$-functors only for local quotient stacks, so we need to introduce this concept and we show that for these stacks any gerbe arises from a projective Hilbert bundle, and the latter is almost uniquely determined by the gerbe. References for this section are $[\mathbf{F H T}],[\mathbf{L T X}]$ and the preprint of Atiyah and Segal $[\mathbf{A S}]$.

Definition 6.1. A differentiable stack $\mathscr{M}$ is called a local quotient stack if there is a covering $\mathscr{U}_{i}$ of $\mathscr{M}$ by open substacks, such that each $\mathscr{U}_{i} \cong\left[U_{i} / G_{i}\right]$, where $G_{i}$ is a compact Lie group acting on a manifold $U_{i}$.

Quite a lot of stacks have this property, a very general result was recently given in $[$ Zun]. Of course if a stack $\mathscr{M}$ is a local quotient stack, then the diagonal $\mathscr{M} \rightarrow \mathscr{M} \times \mathscr{M}$ is proper. We say that $\mathscr{M}$ has proper isotropy. 
By the standard slice theorems (e.g. [DK00] Chapter 2) to be a local quotient stack is a local property as follows (note that we assumed the Lie groups to be compact):

Lemma 6.2. (To be local quotient stack is a local property) Let $\mathscr{M}$ be a local quotient stack, $X \rightarrow \mathscr{M}$ an atlas. Given a point $x \in X$ and $x \in U \subset X$ open there is an open substack $\mathscr{U} \subset \mathscr{M}$ together with a presentation $\mathscr{U} \cong[Y / G]$ where $G$ is a compact Lie group acting on a contractible manifold $Y$, and $a$ commuting diagram:

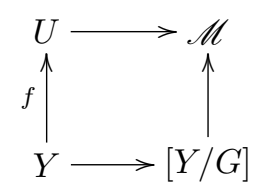

and $x \in \operatorname{Im}(f)$.

Corollary 6.3. Any $S^{1}$-gerbe on a local quotient stack is again a local quotient stack.

Proof of Lemma 6.2. Shrinking $\mathscr{M}$ we may assume that $\mathscr{M} \cong\left[X^{\prime} / G^{\prime}\right]$ is a global quotient stack. Further we may assume that $X=X^{\prime}$, because the projections of the fibered product $X^{\prime} \leftarrow X \times \mathscr{M} X^{\prime} \rightarrow X$ are submersions, thus we may choose a preimage $\tilde{x}$ of $x$ in the fibered product and a local section $X^{\prime} \supset U \rightarrow X^{\prime} \times \mathscr{M} X$ passing through $\tilde{x}$.

But now we can find a contractible slice of the group action, which gibes us a local presentation as $\mathscr{U}=[D / S \operatorname{Stab}(x)]$, where $D$ is a ball and the action of the stabilizer of $x$ comes from the linear action on the tangent space at $x$.

Proof of Corollary 6.3. We may assume $\mathscr{M}=[X / G]$ is a global quotient. Since gerbes on contractible spaces are trivial, we may apply the last lemma to get a covering of $\mathscr{M}$ by open substacks of the form $[Y / H]$ such that the given gerbe is trivial on $Y$. Since $Y$ is contractible, the gerbe is induced form a $S^{1}$-extension of $H$.

To end the section on local quotient stacks, we want to show that for these stacks any $S^{1}$-gerbe is defined by a projective bundle, which can be chosen in an almost canonical way (up to non canonical isomorphism). To this end we first need the concept of a universal Hilbert bundle, as defined in [FHT]. 


\section{Definition 6.4 (Freed, Hopkins, Teleman [FHT])}

A Hilbert bundle $H$ on a differentiable stack $\mathscr{M}$ is called universal if any other Hilbert bundle $H^{\prime}$ is a direct summand of $H$. A universal Hilbert bundle is called local if its restriction to any open substack is universal.

Lemma 6.5 ([FHT] C.3). A universal bundle $H$ on a stack $\mathscr{M}$ has the absorption property: For any Hilbert bundle $H^{\prime}$ on $\mathscr{M}$ there is an isomorphism $H \oplus H^{\prime} \cong H$.

The basic proposition is:

Proposition 6.6 ([FHT $]$ C.4). Let $\mathscr{M}$ be a local quotient stack. Then there exists a universal Hilbert $H$ bundle on $\mathscr{M}$. This bundle is local, and its group of unitary automorphisms is weakly contractible.

We sketch the argument of [FHT]: On manifolds all Hilbert bundles are trivial, because the infinite unitary group $U$ is contractible. Now let $\mathscr{M}$ be a global quotient stack $[X / G](G$ a compact Lie group). Let $\pi: X \rightarrow[X / G]$ be the universal $G$ bundle on $[X / G]$. Then for any Hilbert bundle $\mathscr{H}$ on $[X / G]$ the bundle $\pi^{*} \mathscr{H}$ is trivial, and there is a canonical injection $\mathscr{H} \rightarrow \pi_{*} \pi^{*} \mathscr{H}$, where $\pi_{*}$ means the bundle of fiber wise $L^{2}$ sections. Thus $\pi_{*}$ of the trivial Hilbert bundle on $X$ is a universal bundle which is local.

Now the global automorphisms of this Hilbert bundle are $G$-equivariant maps from $X \rightarrow \mathrm{U}\left(H \otimes L^{2}(G)\right)$, and the space of these maps is contractible ([AS $]$ Proposition A3.1). Thus for a local quotient stack one can glue the local bundles and the result is unique up to isomorphism. Thus it gives a universal bundle on $\mathscr{M}$.

6.1. $S^{1}$-gerbes on local quotient stacks. To see that any $S^{1}$ gerbe arises from a projective bundle one is tempted to use the cohomology sequence coming from the short exact sequence $1 \rightarrow S^{1} \rightarrow \mathrm{U} \rightarrow \mathrm{PU} \rightarrow 1$. Unfortunately there is no nice definition of $H^{2}$ for non-abelian groups, therefore we need some preparations, to get canonical elements in $H^{1}(\mathscr{M}, \mathrm{PU})$.

First we need an absorption property for projective bundles, which I learned from $[\mathbf{A S}]$.

Lemma 6.7. Let $\mathscr{M}$ be any topological stack.

1. The tensor product induces a map

$$
\otimes: H^{1}(\mathscr{M}, \mathrm{U}) \times H^{1}(\mathscr{M}, \mathrm{PU}) \rightarrow H^{1}(\mathscr{M}, \mathrm{PU})
$$


2. The tensor product does not change the induced gerbe, i.e., denote by $\partial$ the boundary map $\partial: H^{1}(\mathscr{M}, \mathrm{PU}) \rightarrow H^{2}\left(\mathscr{M}, \mathscr{S}^{1}\right)$, then for any Hilbert bundle $H$ and any projective bundle $P$ on $\mathscr{M}$ we have $\partial(H \otimes P)=\partial(P)$.

Proof. Any isomorphism $H \otimes H \cong H$ induces a group homomorphism $U \times \mathrm{PU} \rightarrow$ PU. This is well defined up to inner automorphisms of PU.

For the second part we only have to note that the choice of a Hilbert $U$ structure on $P$ also induces one on $H \otimes P$, and this is compatible with the $S^{1}$ action on $\mathrm{U}$, thus the gerbes coming from the obstruction to such a lift are isomorphic.

Definition 6.8. (Atiyah-Segal ${ }^{(3)}[\mathbf{A S}]$ ) A projective Hilbert bundle $P$ (i.e. a PU-Bundle) on a differentiable stack $\mathscr{M}$ has the absorption property if for any Hilbert bundle $H$ on $\mathscr{M}$ there is an isomorphism $H \otimes P \cong P$.

We denote the set of isomorphism classes of projective bundles having the absorption property by $H^{1}(\mathscr{M}, \mathrm{PU})_{a b s}$.

Remark 6.9. If $H_{\text {univ }}$ is a universal Hilbert bundle on a stack $\mathscr{M}$ and $P$ is any projective bundle, then $H_{\text {univ }} \otimes P$ has the absorption property.

Lemma 6.10. Let $\mathscr{M}$ be any differentiable stack. Then the map

$$
H^{1}(\mathscr{M}, \mathrm{PU})_{a b s} \rightarrow H^{2}\left(\mathscr{M}, \mathscr{S}^{1}\right)
$$

is injective.

Proof. Let $P$ be a projective bundle, having the absorption property and let $\pi: \tilde{\mathscr{M}} \rightarrow \mathscr{M}$ be the $S^{1}$-gerbe of Hilbert bundle structures on $P$. Then $\pi^{*} P \cong$ $\mathbb{P}(H)$ for some Hilbert bundle $H$ on $\tilde{\mathscr{M}}$.

Aside on weights: Because $S^{1}$ is canonically contained in the automorphism group of any object of $\tilde{\mathscr{M}}$, it acts on the sections of any Hilbert bundle $\mathscr{H}$ on $\mathscr{M}$. Thus the canonical decomposition of the sheaf of sections of $\mathscr{H}$ induces a decomposition o $\mathscr{H}=\oplus_{i \in \mathbb{Z}} \mathscr{H}_{i}$, according to the characters of $S^{1}$, called weights. Bundles of weight 0 - i.e. bundles for which $\mathscr{H}=\mathscr{H}_{0}$ - are pull-backs of Hilbert bundles on $\mathscr{M}$. Bundles of weight 1 - i.e. $\mathscr{H}=\mathscr{H}_{1}$ - are exactly the bundles, which induce projective bundles on $\mathscr{M}$ whose associated gerbe is $\tilde{\mathscr{M}}$.

Thus in our situation $\mathscr{H}$ is a bundle of weight 1 and we want to show, that it has the absorption property for Hilbert bundles of weight 1 on $\tilde{\mathscr{M}}$. Let $\mathscr{H}^{\prime}$ be an irreducible Hilbert bundle of weight one on $\tilde{\mathscr{M}}$. Then $\mathscr{H} \otimes \mathscr{H}^{\prime, *}$ has

$\overline{{ }^{(3)} \text { In their article }}[\mathbf{A S}]$ this property is called regular, we keep the terminology of [FHT] 
weight 0 , thus $\mathscr{H} \otimes \mathscr{H}^{*} \cong \pi^{*}\left(\mathscr{H}_{\mathscr{M}}\right)$. Since $P$ has the absorption property, we know that $\mathscr{H} \cong \mathscr{H} \otimes \pi^{*}\left(\mathscr{H}^{\prime *}\right) \otimes$. Thus $\mathscr{H} \otimes \mathscr{H} / *$ has a non vanishing section (even countably many linear independent sections), which proves the absorption property.

By uniqueness of universal bundles this shows that $\mathscr{H}$ is determined by the gerbe.

Remark 6.11. If there is a universal Hilbert bundle on $\mathscr{M}$, which is local, then the restriction to open substacks preserves the absorption property. And conversely it is then enough to check this property locally.

Proposition 6.12. Every $S^{1}$-gerbe on a local quotient stack $\mathscr{M}$ comes from a projective bundle. Moreover, the natural map

$$
H^{1}(\mathscr{M}, \mathrm{PU})_{a b s} \rightarrow H^{2}\left(\mathscr{M}, \mathscr{S}^{1}\right)
$$

is an isomorphism.

Proof. Let $\tilde{\mathscr{M}}$ be an $S^{1}$-gerbe on $\mathscr{M}$. By Lemma 6.3 this is again a local quotient stack and therefore it has a universal Hilbert bundle $\tilde{\mathscr{H}}$. As in the previous lemma, we denote the direct summand of weight 1 of $\tilde{\mathscr{H}}$ by $\tilde{\mathscr{H}}_{1}$. This bundle is non-trivial, since it is locally the gerbe is defined by a group extension, thus locally the bundle is non trivial. Thus $\tilde{\mathscr{H}}_{1}$ defines a projective bundle on $\mathscr{M}$, which gives the gerbe.

\section{References}

[AS] M. AtiYah \& G. Segal - Twisted K-Theory, math.KT/0407054.

[Bre94] L. BREEN - On the classification of 2-gerbes and 2-stacks, Astérisque (1994), no. 225 , p. 160 .

[Cra] M. CRAINIC - Differential and algebroid cohomology, van Est isomorphisms, and characteristic classes, math.DG/0008064.

[Del74] P. Deligne - Théorie de Hodge. III, Inst. Hautes Études Sci. Publ. Math. (1974), no. 44, p. 5-77.

[DK00] J. J. DuistermaAT \& J. A. C. KolK - Lie groups, Universitext, SpringerVerlag, Berlin, 2000.

[FHT] D. Freed, M. Hopkins \& C. Teleman - Twisted $K$-Theory and Loop Group Representations I, math.AT/0312155.

[Fri82] E. M. FRIEDLANDER - Étale homotopy of simplicial schemes, Annals of Mathematics Studies, vol. 104, Princeton University Press, Princeton, N.J., 1982 . 
[LMB00] G. Laumon \& L. Moret-Bailly - Champs algébriques, Ergebnisse der Mathematik und ihrer Grenzgebiete. 3. Folge., vol. 39, Springer-Verlag, Berlin, 2000.

[LTX] C. Laurent, J.-L. Tu \& P. XU - Twisted $K$-theory of Differentiable Stacks, math.KT/0306138.

[Zun] N. T. ZUNG - Proper groupoids and moment maps: Linearization, affinity and convexity, math.SG/0407208. 
Mathematisches Institut, Seminars, (Y. Tschinkel, ed.), p. 33-80

Universität Göttingen, 2004-05

\title{
TWISTED $K$-THEORY AND TQFT
}

\section{U. Bunke}

Mathematisches Institut, Universität Göttingen, Bunsenstr. 3-5, 37073

Göttingen, Germany • E-mail : bunke@uni-math.gwdg.de

\section{Schröder}

Mathematisches Institut, Universität Göttingen, Bunsenstr. 3-5, 37073

Göttingen, Germany • E-mail : ischroed@uni-math.gwdg.de

\begin{abstract}
The goal of the present paper is the calculation of the equivariant twisted K-theory of a compact Lie group which acts on itself by conjugations, and elements of a TQFT-structure on the twisted K-groups. These results are originally due to D.S. Freed, M.J. Hopkins and C. Teleman. In this paper we redo their calculations in the framework of topological and differentiable stacks. We also show how moduli spaces of flat connections on surfaces give rise to trivializations of twists.
\end{abstract}

\section{Introduction}

The present paper grew out of a seminar held in the Spring, 2004. The goal of the seminar was to understand the recent paper by Freed, Hopkins, and Teleman [FHT03]. The first main result of [FHT03] that we discussed was the calculation of the twisted $G$-equivariant $K$-theory of $G$, where $G$ is a compact Lie group which acts on itself by conjugation.

While working on details we came to the conclusion that it is worth to develop proofs in a more restricted formalism. The original paper [FHT03] mixes analytic with geometric and topological arguments. We had some difficulties to see that all constructions match in a nice manner.

In the present paper we try to give a proof of this result (which we formulate here as Theorem 2.1) by arguments which are completely embedded in the calculus of smooth stacks. We do not touch the question of the construction of a $K$-theory functor in this framework. Rather we assume that such a functor

May 2005. 
exists and has all necessary functorial properties. Actually we only need local quotient stacks, and the construction of the $K$-theory in this case was sketched in [FHT03] (see also [TXL03] and [AS04]). A verification of all functorial properties, in particular the construction of push-forward maps, is still a gap in the literature.

The way the calculation is set up in the present paper opens the path to generalizations. Since we only do geometric calculations with stacks and use the formal properties of $K$-theory, the method could also be applied to other twisted cohomology theories.

The calculation of the twisted $K$-theory has two basis steps. The first step is the construction of elements of the twisted $K$-theory. In symbols the construction is realized as the map $R_{!} \circ \Phi$, where $\Phi$ is defined in 2.3.4, and the map $R$ is introduced in 2.3. Note that this construction is purely geometric in terms of the calculus of smooth stacks and formal properties of twisted $K$-theory.

The second step is a method to detect elements of the twisted $K$-theory. We will construct an embedding (the map $\Theta$ introduced in 2.4.6, see Theorem 2.17 ) of the twisted $K$-theory into the representation ring of a suitable finite group 2.4.3. This very effective tool was explained to us by C. Teleman.

The second topic of the seminar were elements of a 1+1-dimensional TQFTstructure on the twisted $K$-theory. The identity, the product, and the co-form are induced by natural geometric constructions with stacks associated to the group. Having constructed a basis of the twisted $K$-theory in Theorem 2.1 it is then a natural question to express these TQFT-operations in terms of this basis. The results and sketches of proofs were announced again in [FHT03] and [FHT02]. Here we reproduce the formulas working again completely inside the stack calculus and using only formal properties of $K$-theory. The identity is calculated in Theorem 2.20. The co-form is obtained in Theorem 2.23. Finally, the calculation of the product is stated as Theorem 3.25.

The twisted $K$-theory of a Lie group which acts on itself by conjugation is a module over the representation ring of the Lie group in a natural way. It follows from the calculation that the twisted $K$-theory is a free $\mathbb{Z}$-module and therefore embeds in its complexification. The latter is a module over the complexified group ring. In Theorem 2.19 we show that it is a quotient of the complexified group ring. Actually, equipped with the product and the identity coming from the TQFT-structure, the complexified twisted $K$-theory is a quotient of the complexified representation ring as a ring.

The natural source of the TQFT-structure are correspondences given by moduli spaces of flat connections on surfaces and their boundaries (see (3.15)). In the present paper these correspondences were only employed to construct isomorphisms of twists needed to define the product. 
Indeed, we had difficulties to define the $K$-orientations of the outgoing boundary evaluation maps in a natural way such that they are compatible with glueing.

In Subsection 3.1 we recall the construction of the central extension of the restricted unitary group associated to a polarized Hilbert space. This central extension can be viewed as a source of twists. In 3.2.4 we use this central extension in order define in a natural way twists of the moduli stack of (flat) $G$-connections on any one-dimensional compact closed oriented manifold.

The determinant line bundle over the restricted Grassmannian of the polarized Hilbert space can be used as a source of trivializations of twists. For a compact oriented surface with boundary we have an evaluation map from the moduli stack of flat $G$-connections on the surface to the stack of $G$-connections on the boundary. In Subsection 3.11 we construct a natural trivialization of the pull-back of the twist via the boundary evaluation. In Propositions 3.13 and 3.18 we verify that these trivializations behave functorially with respect to the glueing of surfaces. This approach to twists was partly inspired by the thesis of Posthuma [Pos03].

The missing piece for a completely natural construction of the TQFT using moduli spaces is the compatible orientation of the outgoing boundary evaluation maps. Such a construction is desirable in particular, because it would give a natural explanation for the associativity of the product.

A major topic of [FHT03] is the relation between the equivariant twisted $K$-theory of the Lie group acting on itself by conjugation and the theory of positive energy representations of the associated loop group. Because of lack of time this was not discussed in the seminar and will therefore not be touched upon in the present paper. Another more philosophical reason for this omission is that according to our present knowledge this relation can not be seen purely inside the calculus of stacks. Rather it is based on explicit cycles in order to represent twisted $K$-theory classes in an appropriate model.

While working on this paper we had a very fruitful exchange with C. Teleman. He told us the idea how to detect elements of the twisted $K$-theory groups using the restriction to finite groups. Furthermore this discussion led to the elimination of many stupid mistakes in previous versions of these notes.

In the same seminar J. Heinloth gave an introduction to smooth stacks and gerbes. In the present paper we freely use the language and the notation which was set up in his talks and the review [Hei05]. Further discussions with J. Heinloth during the preparation of the present paper were of great help.

Finally, since this will not be noted again in the text below, let us emphasize that the main theorems discussed in the present paper and the key ideas leading to their verifications are due to Freed, Hopkins, and Teleman. 


\section{Calculation of twisted $K$-theory of Lie groups}

\subsection{Connections, gauge groups, and twists}

2.1.1. Let $G$ be a Lie group. We consider the trivial $G$-principal bundle

$$
P\left(S^{1}\right):=G \times S^{1} \rightarrow S^{1} .
$$

Let $F\left(S^{1}\right)$ denote the space of (flat) connections on $P\left(S^{1}\right)$. The gauge group $G\left(S^{1}\right)$ acts on $F\left(S^{1}\right)$. We consider the topological stack (see [Hei05], Ex. 1.5 and 2.5)

$$
\mathscr{M}:=\left[F\left(S^{1}\right) / G\left(S^{1}\right)\right]
$$

2.1.2. Let

$$
0 \rightarrow U(1) \rightarrow \hat{G}\left(S^{1}\right) \rightarrow G\left(S^{1}\right) \rightarrow 0
$$

be a central extension. It gives rise to a twist (see [Hei05], Ex. 5.4.1)

$$
\tau: \hat{\mathscr{M}} \rightarrow \mathscr{M},
$$

where $\hat{\mathscr{M}}:=\left[F\left(S^{1}\right) / \hat{G}\left(S^{1}\right)\right]$. The goal of the present section is a to formulate the main result about the calculation of the twisted $K$-theory ${ }^{\tau} K(\mathscr{M})$.

2.1.3. Twisted $K$-theory associates to a topological stack $\mathscr{M}$ equipped with a twist $\tau: \hat{\mathscr{M}} \rightarrow \mathscr{M}$ a $\mathbb{Z}$-graded group ${ }^{\tau} K(\mathscr{M})$ in a functorial way. More precisely, if $\tau^{\prime}: \hat{\mathscr{M}}^{\prime} \rightarrow \mathscr{M}^{\prime}$ is another twisted topological stack, $f: \mathscr{M}^{\prime} \rightarrow \mathscr{M}$ is a morphism, and $u: \tau^{\prime} \rightarrow f^{*} \tau$ is an isomorphism of twists, then we have a functorial map $u^{*} f^{*}:{ }^{\tau} K(\mathscr{M}) \rightarrow{ }^{\tau^{\prime}} K\left(\mathscr{M}^{\prime}\right)$. See [FHT03], [TXL03], and [AS04] for a construction a twisted $K$-theory functor. We further assume that twisted $K$-theory admits a Mayer-Vietoris sequence and is a module over the untwisted $K$-theory. Our assumptions about wrong-way maps will be explained in 2.2.9.

2.1.4. We consider $G \subset G\left(S^{1}\right)$ as the subgroup of constant gauge transformations. We assume that $G$ is connected and choose a maximal torus $T \subset G$. Let $\check{T}$ denote the group of homomorphisms $S^{1} \rightarrow T$. We can consider $\check{T} \subset G\left(S^{1}\right)$ naturally. Furthermore let $N_{G}(T)$ be the normalizer of $T$ in $G$ which we also consider as a subgroup of $G\left(S^{1}\right)$. Inside $G\left(S^{1}\right)$ the groups $\check{T}$ and $N_{G}(T)$ generate a semi-direct product

$$
0 \rightarrow \check{T} \rightarrow \check{T} N_{G}(T) \rightarrow N_{G}(T) \rightarrow 0 .
$$

The group of connected components of $\check{T} N_{G}(T)$ is the affine Weyl group $\hat{W}$. It fits into a semi-direct product

$$
0 \rightarrow \check{T} \rightarrow \hat{W} \rightarrow W \rightarrow 0,
$$

where $W:=N_{G}(T) / T$ is the ordinary Weyl group of the pair $(G, T)$. 
2.1.5. Let $\hat{T} \rightarrow T$ be the restriction of the central extension of $G\left(S^{1}\right)$ via the embedding $T \subset G\left(S^{1}\right)$. Let $X(\hat{T})$ denote the group of characters, and let $X_{1}(\hat{T}) \subset X(\hat{T})$ be the subset of those characters which become the identity after restriction to the central $U(1)$.

The torus $T \subset G\left(S^{1}\right)$ is preserved under conjugation by elements of $\check{T} N_{G}(T)$. Therefore $\check{T} N_{G}(T)$ acts on $X(\hat{T})$. In fact, this action preserves $X_{1}(\hat{T})$ and factors over the affine Weyl group.

2.1.6. We call an element $\chi \in X_{1}(\hat{T})$ regular, if its stabilizer in $\hat{W}$ is trivial. Otherwise we call $\chi$ singular. Let $X_{1}^{r e g}(\hat{T})$ denote the set of regular elements.

We call the twist $\tau$ regular, if $\hat{W}$ acts properly on $X_{1}(\hat{T})$ with finitely many orbits.

Let $\check{T} \widehat{N_{G}(T)} \rightarrow \check{T} N_{G}(T)$ be the central extension induced by the restriction of $\hat{G}\left(S^{1}\right) \rightarrow G\left(S^{1}\right)$ to $\check{T} N_{G}(T)$. By further restrictions we obtain central extensions $\widehat{N_{G}(T)}$ and $\widehat{\check{T}}$ of $N_{G}(T)$ and $\check{T}$. We call the twist $\tau$ admissible if $\widehat{N_{G}(T)}$ and $\widehat{\widetilde{T}}$ are trivial.

2.1.7. The main result of the present section is the formulation of the following theorem:

Theorem 2.1. Assume that $G$ is connected, and that $\tau$ is regular and admissible. Then the orbit set $X_{1}^{r e g}(\hat{T}) / \hat{W}$ is the index set of a $\mathbb{Z}$-basis of the free $\mathbb{Z}$-module ${ }^{\tau} K(\mathscr{M})$ in a natural way.

We will finish the proof of this theorem in 2.4.10. After a choice of representatives of the equivalence classes $X_{1}^{r e g}(\hat{T}) / \hat{W}$ the basis elements will be determined uniquely up to a global sign which can be fixed by choosing an orientation of $\operatorname{Lie}(T)$.

2.1.8. Let $[G / G]$ be the quotient stack, where $G$ acts in itself by conjugation. We define a map hol : $\mathscr{M} \rightarrow[G / G]$ which on the level of spaces associates to each connection in $F\left(S^{1}\right)$ its holonomy at $1 \in S^{1}$ measured in the positive direction. On the level of groups it is given by the evaluation $G\left(S^{1}\right) \rightarrow G$ at 1 .

Lemma 2.2. The map hol $: \mathscr{M} \rightarrow[G / G]$ is an equivalence of topological stacks.

Proof. This follows from [Hei05], Ex. 3.3 and the fact that the group $G\left(S^{1}\right)_{0} \subset G\left(S^{1}\right)$ of based gauge transformations (those which evaluate trivially at $\left.1 \in S^{1}\right)$ acts freely and properly on $F\left(S^{1}\right)$ with quotient isomorphic to $G$ via the holonomy map, and $G\left(S^{1}\right) / G_{0}\left(S^{1}\right) \cong G$ via the evaluation. 
The composition $\operatorname{hol}_{*} \tau:=\operatorname{hol} \circ \tau: \hat{\mathscr{M}} \rightarrow[G / G]$ is a twist of $[G / G]$. In this sense Theorem 2.1 provides a calculation of ${ }^{\text {hol }{ }_{*} \tau} K([G / G])$.

2.1.9. We consider a regular and admissible twist $\tau$ of $\mathscr{M}$. Let $I:[G / G] \rightarrow$ $[G / G]$ be the map which is given by $g \mapsto g^{-1}$ on the level of spaces, and by the identity on the level of groups. We call the twist $\tau$ odd, if $I^{*} h o l_{*} \tau \cong-h_{\circ} l_{*} \tau$.

\subsection{Orientations.}

2.2.1. Let $E$ be a real euclidian vector space. By $\operatorname{Cliff}(E)$ we denote the associated complex Clifford algebra. It comes with an embedding of $E \rightarrow \operatorname{Cliff}(E)$ and a $*$-operation. Let $\operatorname{Cliff}(E)^{*}$ denote the group of unitary elements. We define

$$
\operatorname{Pin}^{c}(E):=\left\{x \in \operatorname{Cliff}(E)^{*} \mid x E x^{*}=E\right\} .
$$

This group comes as a central extension

$$
0 \rightarrow U(1) \rightarrow \operatorname{Pin}^{c}(E) \rightarrow O(E) \rightarrow 0
$$

If $E=\mathbb{R}^{n}$, then we write $\operatorname{Pin}^{c}(n):=\operatorname{Pin}^{c}\left(\mathbb{R}^{n}\right)$.

We let $\operatorname{Spin}^{c}(E) \subset \operatorname{Pin}^{c}(E)$ be the pre-image of $S O(E) \subset O(E)$, and set $\operatorname{Spin}^{c}(n):=\operatorname{Spin}^{c}\left(\mathbb{R}^{n}\right)$.

2.2.2. The sequence of groups (2.3) induces a sequence of maps of stacks

$$
[* / U(1)] \rightarrow\left[* / \operatorname{Pin}^{c}(n)\right] \rightarrow[* / O(n)] .
$$

We furthermore have the following pull-backs (see [Hei05], Def. 2.1)

$$
\begin{array}{ccccc}
{\left[* / \operatorname{Spin}^{c}(n)\right]} & \rightarrow & {[* / S O(n)]} & \rightarrow & * \\
\downarrow & & \downarrow & & \downarrow \\
{\left[* / \operatorname{Pin}^{c}(n)\right]} & \rightarrow & {[* / O(n)]} & \rightarrow & {[* /(\mathbb{Z} / 2 \mathbb{Z})]}
\end{array} .
$$

2.2.3. A graded twist of a stack $\mathscr{M}$ will be a pair $(\sigma, \rho)$, where $\sigma: \mathscr{M} \rightarrow$ $[* /(\mathbb{Z} / 2 \mathbb{Z})]$ (is called the grading), and $\rho$ is a twist of $\mathscr{M}$.

Let $E \rightarrow \mathscr{M}$ be a real euclidian vector bundle over a stack (see [Hei05], 2.10). Its frame bundle gives rise to a classifying map $\mathscr{M} \rightarrow[* / O(n)]$. We form the pull-back

$$
\begin{array}{ccc}
\operatorname{Pin}^{c}(E) & \rightarrow & {\left[* / \operatorname{Pin}^{c}(n)\right]} \\
\downarrow & & \downarrow \\
\mathscr{M} & \rightarrow & {[* / O(n)]}
\end{array} .
$$

The stack $\operatorname{Pin}^{c}(E)$ classifies $P i n^{c}$-structures on $E$. It is a twist. 
We compose the map $\mathscr{M} \rightarrow[* / O(n)]$ with $[* / O(n)] \rightarrow[* /(\mathbb{Z} / 2 \mathbb{Z})]$ and obtain the pull-back

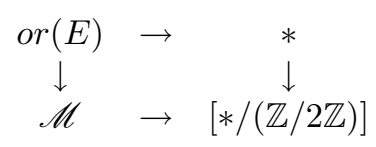

defining the stack or $(E)$ which classifies the orientations of $E$.

In this way the vector bundle $E$ gives rise to a graded twist

$$
\tau(E):=\left(\operatorname{or}(E) \rightarrow \mathscr{M}, \operatorname{Pin}^{c}(E) \rightarrow \mathscr{M}\right) .
$$

2.2.4. The graded twists of a stack form a two-category. There is a natural notion of a sum of graded twists such that there is a natural isomorphism $\tau\left(E_{0} \oplus E_{1}\right) \cong \tau\left(E_{0}\right)+\tau\left(E_{1}\right)$. This sum differs from the component-wise sum. In fact, the isomorphism classes of twists are classified by a group $G T W(\mathscr{M})$ which sits in a non-trivial extension

$$
0 \rightarrow H^{3}(\mathscr{M}, \mathbb{Z}) \rightarrow G T W(\mathscr{M}) \rightarrow H^{1}(\mathscr{M}, \mathbb{Z} / 2 \mathbb{Z}) \rightarrow 0
$$

(see the discussion in [AS04] and [Hei05], Remark 5.9).

2.2.5. Let $f: \mathscr{M} \rightarrow \mathscr{N}$ be a representable smooth map of smooth stacks. A factorization of $f$ into a smooth embedding and a smooth submersions gives rise to a normal bundle (see [Hei05], Def. 4.10) which is a $\mathbb{Z} / 2 \mathbb{Z}$-graded vector bundle $E=E^{+} \oplus E^{-}$over $\mathscr{M}$. We define $\tau(E):=\tau\left(E^{+}\right)-\tau\left(E^{-}\right)$. Let $E^{\prime}$ be a normal bundle obtained by a different factorization. Then using the diagonal embedding we get bundles $A, B$ over $\mathscr{M}$ such that $E \oplus A \oplus(-A)$ and $E^{\prime} \oplus B \oplus(-B)$ are canonically isomorphic. In particular, we get a natural isomorphism $\tau(E) \cong \tau\left(E^{\prime}\right)$.

2.2.6. Let $f: \mathscr{M} \rightarrow \mathscr{N}$ be a representable smooth map which admits factorizations in smooth embeddings and submersions. Let $\tau$ be a graded twist of $\mathscr{N}$.

Definition 2.4. A $\tau$-K-orientation of $f$ is a coherent choice of isomorphisms

$$
f^{*} \tau \stackrel{\sim}{\rightarrow} \tau(E)
$$

of twists for all normal bundles $E$ of $f$ given by some factorization.

Coherence is understood here with respect to the natural isomorphisms (see 2.2.5) of twists associated to the normal bundles given by different factorizations of $f$. Note that a $\tau$ - $K$-orientation is determined by the isomorphism $f^{*} \tau \stackrel{\sim}{\rightarrow} \tau(E)$ for one choice of $E$. 


\subsubsection{Let}

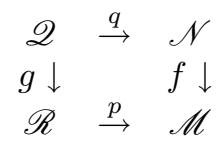

be a cartesian diagram of smooth stacks, where $f$ is representable and has a factorization with normal bundle $E$. Then $g$ is representable and also has a factorization with normal bundle $q^{*} E$. If $f$ is $\tau$ - $K$-oriented, then $g$ has an induced $p^{*} \tau$ - $K$-orientation.

2.2.8. Let us consider a composition

$$
\mathscr{R} \stackrel{g}{\rightarrow} \mathscr{N} \stackrel{f}{\rightarrow} \mathscr{M} .
$$

If $E$ and $F$ are normal bundles (for some factorizations) of $f$ and $g$, then there is a factorization of $f \circ g$ with a normal bundle $g^{*} E \oplus F$. If $f$ is $\tau_{0}$-K-oriented, and $g$ is $f^{*} \tau_{1}$-oriented, then $f \circ g$ is naturally $\tau_{0}+\tau_{1}$ oriented. Vice versa, if $f$ and $f \circ g$ are $K$-oriented, then so is canonically $g$.

2.2.9. We assume that the twisted $K$-theory functor admits functorial wrongway maps for twisted- $K$-oriented proper maps. More precisely, if $f: \mathscr{M} \rightarrow \mathscr{N}$ is a smooth proper (see [Hei05], Def. 2.8) map between stacks which is $\tau-K$ oriented for some twist $\tau$ of $\mathscr{N}$, and if $\sigma$ is a further twist of $\mathscr{N}$, then we have a wrong-way map

$$
f_{!}:{ }^{*} \sigma+f^{*} \tau(\mathscr{M}) \rightarrow{ }^{\sigma} K(\mathscr{N}) .
$$

We assume functoriality with respect to compositions and compatibility with cartesian diagrams. Furthermore, we require a projection formula for the module structure of the twisted $K$-theory over the untwisted $K$-theory.

2.2.10. Let $G$ be a connected compact Lie group. We consider $G$ as a $G \times G$ space with with the action $(a, b) g=a g b^{-1}$.

The group $G$ acts on its Lie algebra $\operatorname{Lie}(G)$ by the adjoint representation. We let $G \times G$ act on $\operatorname{Lie}(G)$ via the projection onto the second factor.

We obtain a vector bundle $[\operatorname{Lie}(G) / G \times G] \rightarrow[* / G \times G]$. Let $\tau(G)$ be the corresponding obstruction twist as in 2.2.4. In fact, since $G$ is connected we do not need the grading.

We will fix once and for all an orientation of $\operatorname{Lie}(G)$. It induces an orientation of $G$. Since $G$ is connected the orientation covering $\operatorname{or}(\operatorname{Lie}(G)) \rightarrow[* / G \times G]$ is trivialized.

2.2.11.

Lemma 2.5. The map $q:[G / G \times G] \rightarrow[* / G \times G]$ has a natural $-\tau(G)$ K-orientation. 
Proof. The normal bundle of $q$ is the tangent bundle $[T G / G] \rightarrow[G / G]$ placed in degree one. We trivialize the tangent bundle $T G \cong G \times \operatorname{Lie}(G)$ using the left action. The action of $\left(g_{1}, g_{2}\right) \in G \times G$ on $(h, X) \in G \times \operatorname{Lie}(G)$ is then given by $\left(g_{1} h g_{2}^{-1}, \operatorname{Ad}\left(g_{2}\right)(X)\right)$. Therefore we obtain an isomorphism $[T G / G \times G] \cong q^{*}[\operatorname{Lie}(G) / G \times G]$. This gives the natural $-\tau(G)$ - $K$-orientation of $p$.

2.2.12. Let $d:[* / G] \rightarrow[* / G \times G]$ be given by the diagonal embedding $G \rightarrow$ $G \times G$. Then we have a cartesian diagram

$$
\begin{array}{cccc}
{[G / G]} & \rightarrow & {[G / G \times G]} \\
p \downarrow & & q \downarrow \\
{[* / G]} & \stackrel{d}{\rightarrow} & {[* / G \times G]}
\end{array} .
$$

The $-\tau(G)$ - $K$-orientation of $q$ induces a $-\sigma(G)$ - $K$-orientation of $p:[G / G] \rightarrow$ $[* / G]$, where $\sigma(G):=d^{*} \tau(G)$. We consider the sequence

$$
[* / G] \stackrel{i}{\rightarrow}[G / G] \stackrel{p}{\rightarrow}[* / G]
$$

The canonical $K$-orientation of the composition $p \circ i=$ id and the $-\sigma(G)-K$ orientation of $p$ induce a $p^{*} \sigma(G)$-K-orientation of $i$.

2.2.13. The normal bundle of the map $* \rightarrow[* / G]$ is $\operatorname{Lie}(G) \rightarrow *$ placed in degree one. We have already fixed an orientation in 2.2.10. The unique Spin $^{c}$ structure on the vector bundle $\operatorname{Lie}(G) \rightarrow *$ induces the $K$-orientation of

$$
* \rightarrow[* / G] \text {. }
$$

2.2.14. Twists of $[* / G]$ are classified by $H^{3}([* / G], \mathbb{Z}) \cong H^{3}(B G, \mathbb{Z})$ (see [Hei05], Prop. 5.8). In fact, the class of $\sigma(G)$ is two-torsion since it comes from a finite-dimensional vector bundle. Note that $H^{3}(B G, \mathbb{Z})_{\text {tors }} \cong$ $\operatorname{Ext}\left(H_{2}(B G, \mathbb{Z}), \mathbb{Z}\right)$, and that $H_{2}(B G, \mathbb{Z}) \cong \pi_{1}(G)$. Therefore, if we assume that 2 does not divide the order of $\pi_{1}(G)_{\text {tors }}$, then $\sigma(G)$ is trivial.

Note further, that the isomorphism classes of trivializations of $\sigma(G)$ form a $H^{2}(B G, \mathbb{Z})$-torsor $\left([\right.$ Hei05], Remark 5.7$)$, and that $H^{2}(B G, \mathbb{Z}) \cong$ $\operatorname{Hom}\left(\pi_{1}(\mathbb{Z}), \mathbb{Z}\right)$ (since $G$ is connected). Thus, if we assume that $\pi_{1}(G)$ is finite, then $\sigma(G)$ is trivial in a unique way.

2.2.15. Let $T \subset G$ be a maximal torus and $N_{G}(T)$ be its normalizer.

Lemma 2.6. The map $q:\left[T / N_{G}(T)\right] \rightarrow[* / G]$ has a natural $-\sigma(G)-K$ orientation. 
Proof. We represent (see [Hei05], Ex. 3.3) this map as

$$
\left[\left(G \times N_{G}(T) T\right) / G\right] \rightarrow[* / G] .
$$

Then we can write the tangent bundle of $G \times_{N_{G}(T)} T$ as associated vector bundle

$$
(G \times T) \times_{N_{G}(T)}\{(\operatorname{Lie}(G) / \operatorname{Lie}(T)) \oplus \operatorname{Lie}(T)\} .
$$

Since the representation of $N_{G}(T)$ on $\operatorname{Lie}(G) / \operatorname{Lie}(T) \oplus \operatorname{Lie}(T) \cong \operatorname{Lie}(G)$ extends to the adjoint representation of $G$ we have an isomorphism of $G$ equivariant bundles

$$
(G \times T) \times_{N_{G}(T)}\{(\operatorname{Lie}(G) / \operatorname{Lie}(T)) \oplus \operatorname{Lie}(T)\} \cong\left(G \times_{N_{G}(T)} T\right) \times \operatorname{Lie}(G) .
$$

Therefore we can identify the vertical bundle of $q$ with the pull-back by $q$ of Lie $(G) \rightarrow *$ as $G$-equivariant bundles. This provides the natural $-\sigma(G)-K$ orientation of $q$.

2.2.16. We consider the composition

$$
\left[T / N_{G}(T)\right] \stackrel{R}{\rightarrow}[G / G] \stackrel{\pi}{\rightarrow}[* / G],
$$

where $R$ is induced by the obvious embeddings on the level of spaces and groups. Now $\pi$ has a natural $-\sigma(G)$ - $K$-orientation by Lemma 2.5 , and the composition $\pi \circ R$ has a natural $-\sigma(G)$ - $K$-orientation by Lemma 2.6. It follows that the map $R$ has a natural $K$-orientation.

\subsection{Construction of twisted $K$-theory .}

2.3.1. We have an embedding Lie $(T) \rightarrow F\left(S^{1}\right)$ as constant connections. The group $\check{T} N_{G}(T) \subset G\left(S^{1}\right)$ preserves the image and thus acts on Lie $(T)$. Let $\mathscr{T}:=\left[\operatorname{Lie}(T) / \check{T} N_{G}(T)\right]$. Then we have a map of stacks

$$
R: \mathscr{T} \rightarrow \mathscr{M} \text {. }
$$

Let $\breve{T N_{G}(T)} \rightarrow \check{T} N_{G}(T)$ denote the restriction of the central extension $\hat{G}\left(S^{1}\right) \rightarrow G\left(S^{1}\right)$ and set

$$
\hat{\mathscr{T}}:=\left[\operatorname{Lie}(T) / \widetilde{T} \widehat{N_{G}(T)}\right] .
$$

Then we have the twist (see [Hei05], Remark 5.5.3 for the pull-back of a twist)

$$
R^{*} \tau: \hat{\mathscr{T}} \rightarrow \mathscr{T} .
$$

We consider the $\hat{T}$-principal bundle $h: \check{T} \widehat{N_{G}(T)} \rightarrow \hat{W}$, where $h$ is the projection to the group of connected components. We let $L^{2}(h) \rightarrow \hat{W}$ be the bundle of Hilbert spaces such that its fibre $L^{2}(h)_{\hat{w}}$ over $\hat{w} \in \hat{W}$ is $L^{2}\left(h^{-1}(\hat{w})\right)$. 
Note that $\hat{T}$ acts on $L^{2}(h)_{\hat{w}}$ via the right action on the fibre. We further define the line bundle $L \rightarrow \hat{W} \times X_{1}(\hat{T})$ such that the fibre $L_{(\hat{w}, \chi)}$ over $(\hat{w}, \chi)$ is the $\chi$-isotypic component $L^{2}(h)_{\hat{w}}(\chi) \subset L^{2}(h)_{\hat{w}}$ as a $\hat{T}$-representation.

We define an action of

$$
\widetilde{T} \widehat{N_{G}(T)} \times \check{T} \widehat{N_{G}(T)}
$$

on

$$
L \rightarrow \hat{W} \times X_{1}(\hat{T})
$$

as follows. Let $f \in L^{2}(h)_{\hat{w}}(\chi)=L_{(\hat{w}, \chi)}$. Then we define

$$
\left(\hat{n}_{1}, \hat{n}_{2}\right)(f)(\hat{g}):=f\left(\hat{n}_{1}^{-1} \hat{g} \hat{n}_{2}\right) \text { for all } \hat{g} \in h^{-1}\left(\hat{n}_{1} \hat{w} \hat{n}_{2}^{-1}\right) .
$$

We calculate that

$$
\begin{aligned}
\left(\hat{n}_{1}, \hat{n}_{2}\right)(f)(\hat{g} \hat{t}) & =f\left(\hat{n}_{1}^{-1} \hat{g} \hat{t} \hat{n}_{2}\right)=f\left(\hat{n}_{1}^{-1} \hat{g} \hat{n}_{2} \hat{n}_{2}^{-1} \hat{t} \hat{n}_{2}\right) \\
& =f\left(\hat{n}_{1}^{-1} \hat{g} \hat{n}_{2}\right) \chi\left(\hat{n}_{2}^{-1} \hat{t} \hat{n}_{2}\right)=\left(\hat{n}_{2} \chi\right)(\hat{t})\left(\hat{n}_{1}, \hat{n}_{2}\right)(f)(\hat{g}) .
\end{aligned}
$$

It follows that $\left(\hat{n}_{1}, \hat{n}_{2}\right)(f) \in L_{\left(\hat{n}_{1} \hat{w} \hat{n}_{2}^{-1}, \hat{n}_{2} \chi\right)}$. Therefore the projection of $L$ becomes equivariant if we let $\breve{T} \widehat{N_{G}(T)} \times \check{T} \widehat{N_{G}(T)}$ act on the base $\hat{W} \times X_{1}(\hat{T})$ by $\left(\hat{n}_{1}, \hat{n}_{2}\right)(\hat{w}, \chi):=\left(\hat{n}_{1} \hat{w} \hat{n}_{2}^{-1}, \hat{n}_{2} \chi\right)$. Since we assume that $\tau$ is admissible we can choose a split $\hat{W} \rightarrow \check{T} \widehat{N_{G}(T)}$ of $h$ which is a homomorphism. We let $\hat{W}$ act on $L \rightarrow \hat{W} \times X_{1}(\hat{T})$ via its embedding into the left factor of $\check{T} \widehat{N_{G}(T)} \times \check{T} \widehat{N_{G}(T)}$ given by the split. It acts freely, and the quotient is a certain $\check{T} \widehat{N_{G}(T)}$-equivariant line bundle

$$
\bar{L} \rightarrow X_{1}(\hat{T}) .
$$

Note that the central $U(1) \subset \check{T} \widehat{N_{G}(T)}$ acts on the fibres of $\bar{L}$ by the identity character $(\bar{L}$ is of weight one in the language of [Hei05], Lemma 5.6) Therefore we can consider the unit sphere bundle

$$
\left[U(\bar{L}) / \check{T} \widehat{N_{G}(T)}\right] \rightarrow\left[X_{1}(\hat{T}) / \check{T} \widehat{N_{G}(T)}\right]
$$

as a trivialization of the twist

$$
\left[X_{1}(\hat{T}) / \check{T} \widehat{N_{G}(T)}\right] \rightarrow\left[X_{1}(\hat{T}) / \check{T} N_{G}(T)\right] .
$$

We consider the space $\operatorname{Lie}(T) \times X_{1}(\hat{T})$ with the diagonal action of $\check{T} N_{G}(T)$. It gives rise to the stack

$$
\mathscr{S}:=\left[\operatorname{Lie}(T) \times X_{1}(\hat{T}) / \check{T} N_{G}(T)\right] .
$$

The projection to the first factor induces a map $p: \mathscr{S} \rightarrow \mathscr{T}$. The pull-back

$$
p^{*} R^{*} \tau: \hat{\mathscr{S}} \rightarrow \mathscr{S}
$$


is given by

$$
\left.\hat{\mathscr{S}}:=\left[\operatorname{Lie}(T) \times X_{1}(\hat{T}) / \check{T} \widehat{N_{G}(T)}\right)\right] .
$$

It is trivialized (see again [Hei05], Lemma 5.6) by the $U(1)$-bundle

$$
\left.\hat{\operatorname{pr}}_{2}^{*}\left[U(\bar{L}) / \check{T} \widehat{N_{G}(T)}\right] \rightarrow\left[\operatorname{Lie}(T) \times X_{1}(\hat{T}) / \check{T} \widehat{N_{G}(T)}\right)\right]
$$

where

$$
\left.\hat{\mathrm{pr}_{2}}:\left[\operatorname{Lie}(T) \times X_{1}(\hat{T}) / \check{T} \widehat{N_{G}(T)}\right] \rightarrow\left[X_{1}(\hat{T}) / \check{T} \widehat{N_{G}(T)}\right)\right]
$$

denotes the projection.

2.3.2. Let

$$
\overline{\mathscr{S}}:=\left[\operatorname{Lie}(T) \times X_{1}(\hat{T}) / \hat{W}\right]
$$

and $m: \mathscr{S} \rightarrow \overline{\mathscr{S}}$ be the (non-representable) map of stacks induced by the projection $h: \check{T} N_{G}(T) \rightarrow \hat{W}$. Finally we consider the stack $\mathscr{I}:=\left[X_{1}(\hat{T}) / \hat{W}\right]$ and let $r: \overline{\mathscr{S}} \rightarrow\left[X_{1}(\hat{T}) / \hat{W}\right]$ be induced by the projection onto the second factor. We now consider the diagram

$$
\mathscr{T} \stackrel{p}{\leftarrow} \mathscr{S} \stackrel{m}{\rightarrow} \overline{\mathscr{S}} \stackrel{r}{\rightarrow} \mathscr{I} .
$$

2.3.3. By our non-degeneracy assumption $\check{T} \subset \hat{W}$ acts freely on $X_{1}(T)$. Therefore we have a diagram

$$
\begin{array}{ccc}
\overline{\mathscr{S}} & \stackrel{r}{\rightarrow} & \mathscr{I} \\
\cong \downarrow & & \cong \downarrow \alpha \\
{\left[\left(\operatorname{Lie}(T) \times X_{1}(\hat{T}) / \check{T}\right) / W\right]} & \stackrel{\tilde{r}}{\rightarrow} & {\left[\left(X_{1}(\hat{T}) / \check{T}\right) / W\right]}
\end{array}
$$

(see [Hei05], Ex. 3.3 for the vertical isomorphisms). Now $\tilde{r}$ is the projection of a vector bundle with fibre $\operatorname{Lie}(T)$. By 2.2.4 it gives rise to a graded twist $-\tilde{\rho}$ of $\left[\left(X_{1}(\hat{T}) / \check{T}\right) / W\right]$. We let $\rho:=\alpha^{*} \tilde{\rho}$ be the corresponding graded twist of $\mathscr{I}$.

2.3.4. The projection $r$ is now naturally $\rho$-K-oriented. Moreover, $r$ ! : $K_{c}(\overline{\mathscr{S}}) \rightarrow^{\rho} K(\mathscr{I})$ is an isomorphism. Its inverse is the twisted Thom isomorphism. The subscript ${ }_{c}$ stands for proper support over $p$. Since the fibres of $p$ are discrete this map is canonically $K$-oriented. We define

$$
\Phi:=p_{!} \circ\left(t^{-1}\right)^{*} \circ m^{*} \circ\left(r_{!}\right)^{-1}:{ }^{\rho} K(\mathscr{I}) \rightarrow{ }^{R^{*} \tau} K(\mathscr{T}) .
$$

Proposition 2.7. The map $\Phi$ is an isomorphism of groups.

Proof. It suffices to show that

$$
p_{!} \circ\left(t^{-1}\right)^{*} \circ m^{*}: K_{c}(\overline{\mathscr{S}}) \rightarrow{ }^{R^{*}} K(\mathscr{T})
$$


is an isomorphism. We have an equivalence

$$
\exp : \mathscr{T}=\left[\operatorname{Lie}(T) / \check{T} N_{G}(T)\right] \stackrel{\sim}{\rightarrow}\left[T / N_{G}(T)\right]
$$

which is given by the exponential map on the level of spaces, and by the projection $\check{T} N_{G}(T) \rightarrow N_{G}(T)$ on the level of groups. We prove the proposition by localization over open sub-stacks of $\left[T / N_{G}(T)\right]$ and the Mayer-Vietoris principle. Since $\left[T / N_{G}(T)\right]$ has contractible slices we can in fact reduce to points.

2.3.5. So let $t \in T$ and $N_{G}(T)_{t} \subset N_{G}(T)$ be the stabilizer. Then the local model is the diagram of stacks

$$
\left[\check{T} / \check{T} N_{G}(T)_{t}\right] \stackrel{p}{\leftarrow}\left[\check{T} \times X_{1}(\hat{T}) / \check{T} N_{G}(T)_{t}\right] \stackrel{m}{\rightarrow}\left[\check{T} \times X_{1}(\hat{T}) / \hat{W}_{t}\right],
$$

where $\hat{W}_{t}:=\check{T} N_{G}(T)_{t} / T \subset \hat{W}$, and we have identified $\exp ^{-1}(\{t\}) \subset \operatorname{Lie}(T)$ with $\check{T}$.

The restriction of the twist to the local model is given by

$$
\tau_{t}:\left[\check{T} / \check{T} \widehat{N_{G}(T)_{t}}\right] \rightarrow\left[\check{T} / \check{T} N_{G}(T)_{t}\right]
$$

It can be trivialized. We let $N_{G}(T)_{t}$ act from the right on $\left.\check{T} \widehat{N_{G}(T}\right)_{t}$ via a split $\left.N_{G}(T)_{t} \rightarrow \check{T} \widehat{N_{G}(T}\right)_{t}$. Such a split exists by our assumption that the original twist $\tau$ is admissible. The quotient $\left.\check{T} \widehat{N_{G}(T}\right)_{t} / N_{G}(T)_{t}$ is an $U(1)$-bundle $C \rightarrow \check{T}$. Via the left multiplication it is $\left.\check{T} \widehat{N_{G}(T}\right)_{t}$-equivariant. The bundle $C$ gives the trivialization of the restricted twist $\tau_{t}$. We will use this trivialization in order to identify

$$
{ }^{\tau_{t}} K\left(\left[\check{T} / \check{T} N_{G}(T)_{t}\right]\right) \cong K\left(\left[\check{T} / \check{T} N_{G}(T)_{t}\right]\right) .
$$

Let $\left.U \rightarrow\left[X_{1}(\hat{T}) / \check{T} \widehat{N_{G}(T}\right)_{t}\right]$ denote the restriction of $\left.\left[U(\bar{L}) / \check{T} \widehat{N_{G}(T}\right)\right]$ (see ??) to the local model. We form the $\check{T} N_{G}(T)$-equivariant $U(1)$-bundle $V:=$ $\hat{p}^{*} C^{*} \otimes \hat{p r}_{2}^{*} U$, where

$$
\left.\hat{p}:\left[\check{T} \times X_{1}(\hat{T}) / \check{T} \widehat{N_{G}(T)_{t}}\right] \rightarrow\left[\check{T} / \check{T} \widehat{N_{G}(T)}\right)_{t}\right]
$$

is the map induced by the first projection $p$, and

$$
\left.\hat{\mathrm{pr}_{2}}:\left[\check{T} \times X_{1}(\hat{T}) / \check{T} \widehat{N_{G}(T)}\right)_{t}\right] \rightarrow\left[X_{1}(\hat{T}) / \check{T} \widehat{N_{G}(T)_{t}}\right]
$$

is induced by the second projection. We denote by

$$
V^{\otimes}: K\left(\left[\check{T} \times X_{1}(\hat{T}) / \check{T} N_{G}(T)_{t}\right]\right) \rightarrow K\left(\left[\check{T} \times X_{1}(\hat{T}) / \check{T} N_{G}(T)_{t}\right]\right)
$$

the operation given by the tensor product with the line bundle associated to $V$. We then must show that

$$
p_{!} \circ V^{\otimes} \circ m^{*}: K\left(\left[\check{T} \times X_{1}(\hat{T}) / \hat{W}_{t}\right]\right) \rightarrow K\left(\left[\check{T} / \check{T} N_{G}(T)_{t}\right]\right)
$$


is an isomorphism. The candidate for the inverse is $T_{i n v} \circ\left(V^{\otimes}\right)^{-1} \circ p^{*}$, where

$$
T_{i n v}: K\left(\left[\check{T} \times X_{1}(\hat{T}) / \check{T} N_{G}(T)_{t}\right]\right) \rightarrow K\left(\left[\check{T} \times X_{1}(\hat{T}) / \hat{W}_{t}\right]\right)
$$

takes the sub-bundle of $T$-invariants.

2.3.6. In the following calculation we denote by $V, U$, and $C$ the complex line bundles instead of the underlying $U(1)$-bundles. We start with the composition $A:=T_{i n v} \circ\left(V^{\otimes}\right)^{-1} \circ p^{*} \circ p_{!} \circ V^{\otimes} \circ m^{*}$. All these operations can be applied on the level of vector bundles. We consider a vector bundle $X \rightarrow\left[\check{T} \times X_{1}(\hat{T}) / \hat{W}\right]$. We will show that $A(X) \cong X$. Indeed (where the dots indicate a straight-forward calculation)

$$
\begin{aligned}
A(X) & \cong T_{i n v}\left(\hat{p}^{*} C \otimes \hat{p r}_{2}^{*} U^{*} \otimes p^{*} \circ p_{!}\left(\hat{p}^{*} C^{*} \otimes \hat{p r}_{2}^{*} U \otimes m^{*}(X)\right)\right) \\
& \cong T_{i n v}\left(\hat{\mathrm{pr}} \hat{2}_{2}^{*}\left(U^{*}\right) \otimes \hat{p}^{*} \circ \hat{p}_{!}\left(\hat{p r}_{2}^{*}(U) \otimes m^{*}(X)\right)\right) \\
& \cdots \\
& \cong X .
\end{aligned}
$$

We now consider the composition $B:=p_{!} \circ V^{\otimes} \circ m^{*} \circ T_{i n v} \circ\left(V^{\otimes}\right)^{-1} \circ p^{*}$. We again calculate on the level of vector bundles $X \rightarrow\left[\check{T} / \check{T} N_{G}(T)_{t}\right]$. We indeed have

$$
\begin{aligned}
B(X) & \cong p_{!}\left(\hat{p}^{*} C^{*} \otimes \hat{p r}_{2}^{*}(U) \otimes m^{*} \circ T_{i n v}\left(\hat{p}^{*} C \otimes \hat{p r}_{2}^{*} U^{*} \otimes p^{*} X\right)\right) \\
& \cdots X \\
& \cong
\end{aligned}
$$

This finishes the proof of Proposition 2.7.

2.3.7. Observe (see 2.2.16) that $R: \mathscr{T} \rightarrow \mathscr{M}$ is naturally $K$-oriented so that we can consider the induction map

$$
R_{!}:{ }^{R^{*} \tau} K(\mathscr{T}) \rightarrow{ }^{\tau} K(\mathscr{M}) .
$$

Proposition 2.8. $O{ }^{\tau} K(\mathscr{M})$ we have $R ! \circ R^{*}=$ id.

Proof. We have equivalences of stacks

$$
\mathscr{T} \stackrel{\exp }{\cong}\left[T / N_{G}(T)\right] \stackrel{i n d}{\cong}\left[G \times_{N_{G}(T)} T / G\right]
$$

and

$$
\mathscr{M} \stackrel{\text { hol }}{\cong}[G / G],
$$

where ind stands for induction (see [Hei05], Ex. 3.3). Using these equivalences we replace $R$ by the equivalent map

$$
R:\left[\left(G \times{ }_{N_{G}(T)} T\right) / G\right] \rightarrow[G / G]
$$


(which we denote by the same symbol for simplicity). Thus $R$ is represented by the $G$-equivariant map $(g, t) \mapsto g t g^{-1}$. We can now cover $G$ by $G$-equivariant slices $U$ on which the twist is trivializable. We show that $R_{!} \circ R^{*}$ is an isomorphism, locally. Then we argue by the Mayer-Vietoris principle.

2.3.8. In fact we can use contractible slices. The model of the map between the slices at $t \in T$ is

$$
R_{t}:\left[\left(G \times_{N_{G}(T)_{t}} \operatorname{Lie}(T)\right) / G\right] \rightarrow\left[\left(G \times_{G_{t}} \operatorname{Lie}\left(G_{t}\right)\right) / G\right] .
$$

We can obtain the map $R_{t}$ by induction of

$$
S_{t}:\left[\left(G_{t} \times_{N_{G}(T)_{t}} \operatorname{Lie}(T)\right) / G_{t}\right] \rightarrow\left[\operatorname{Lie}\left(G_{t}\right) / G_{t}\right]
$$

from $G_{t}$ to $G$. Thus we must show that $\left(S_{t}\right) ! \circ S_{t}^{*}$ is an isomorphism.

2.3.9. By homotopy invariance and induction isomorphisms we have

$K\left(\left[\left(G_{t} \times_{N_{G}(T)_{t}} \operatorname{Lie}(T)\right) / G_{t}\right]\right) \cong K\left(\left[G_{t} / N_{G}(T)_{t}\right]\right) \cong K\left(\left[* / N_{G}(T)_{t}\right]\right) \cong R\left(N_{G}(T)_{t}\right)$

and

$$
K\left(\left[\operatorname{Lie}\left(G_{t}\right) / G_{t}\right]\right) \cong K\left(\left[* / G_{t}\right]\right) \cong R\left(G_{t}\right) .
$$

With this identification $S_{t}^{*}: R\left(G_{t}\right) \rightarrow R\left(N_{G}(T)_{t}\right)$ is just the usual restriction. In particular we know that this map is injective. In fact this is part of the assertion of Proposition 2.8 in the untwisted case and therefore a part of a $K$-theoretic version of the Borel-Weyl-Bott theorem which we assume as wellknown. It therefore suffices to show that $S_{t}^{*}\left(S_{t}\right) ! S_{t}^{*}=S_{t}^{*}$. Now $S_{t}^{*}\left(S_{t}\right)$ ! is the multiplication with the Euler class of the normal bundle $N$ of $S_{t}$. We will show that the Euler class is equal to one.

2.3.10. We consider the following diagram of $G_{t}$-spaces.

$$
\begin{array}{ccc}
G_{t} \times N_{G}(T)_{t} \operatorname{Lie}(T) & \stackrel{S_{t}}{\rightarrow} & \operatorname{Lie}\left(G_{t}\right) \\
\alpha \downarrow & & \beta \downarrow \\
G_{t} / N_{G}(T)_{t} & \stackrel{\delta}{\rightarrow} & *
\end{array} .
$$

Let $N\left(S_{t}\right), N(\alpha), N(\beta), N(\delta)$ denote the $K_{G_{t}}$-classes of normal bundles of the corresponding maps. Then we have

$$
N\left(S_{t}\right)+S_{t}^{*} N(\beta)=N(\alpha)+\alpha^{*} N(\delta) .
$$

Note that $\beta, \alpha, \delta$ are submersions. It is therefore easy to read-off the normal bundles as the inverses of the vertical bundles. We get

$$
\begin{aligned}
N(\alpha) & =-\alpha^{*}\left[G_{t} \times_{N_{G}(T)_{t}} \operatorname{Lie}(T)\right] \\
N(\beta) & =-\beta^{*}\left[\operatorname{Lie}\left(G_{t}\right)\right] \\
N(\delta) & =-\left[G_{t} \times_{N_{G}(T)}\left(\operatorname{Lie}\left(G_{t}\right) / \operatorname{Lie}(T)\right)\right] .
\end{aligned}
$$


Note that

$$
\begin{aligned}
\alpha^{*}\left[G_{t} \times_{N_{G}(T)_{t}} \operatorname{Lie}(T)\right]+\alpha^{*}\left[G_{t} \times_{N_{G}(T)}\left(\operatorname{Lie}\left(G_{t}\right) / \operatorname{Lie}(T)\right)\right] \\
=\alpha^{*}\left[G_{t} \times_{N_{G}(T)_{t}} \operatorname{Lie}\left(G_{t}\right)\right] \\
=\left[G_{t} / N_{G}(T)_{t} \times \operatorname{Lie}\left(G_{t}\right)\right] \\
=S_{t}^{*} \beta^{*}\left[\operatorname{Lie}\left(G_{t}\right)\right]
\end{aligned}
$$

In view of 2.9 this implies $N\left(S_{t}\right)=0$. Hence its Euler class is the identity. This finishes the proof of Proposition 2.8

\subsubsection{The composition}

$$
\Phi^{-1} \circ R^{*}:{ }^{\tau} K(\mathscr{M}) \rightarrow{ }^{\rho} K(\mathscr{I})
$$

represents ${ }^{\tau} K(\mathscr{M})$ as a direct summand of ${ }^{\rho} K(\mathscr{I})$. In order to determine this summand we must calculate the kernel of

$$
R ! \circ \Phi:{ }^{\rho} K(\mathscr{I}) \rightarrow{ }^{\tau} K(\mathscr{M}) .
$$

2.3.12. Let $\mathscr{I}=\mathscr{I}^{r e g} \cup \mathscr{I}^{\text {sing }}$ be induced by the decomposition of $X_{1}(\hat{T})$ into regular and singular characters. Let $\rho_{s}: \hat{\mathscr{I}}^{\text {sing }} \rightarrow \mathscr{I}^{\text {sing }}$ and $\rho_{r}: \hat{\mathscr{I}}^{\text {reg }} \rightarrow \mathscr{I}^{\text {reg }}$ be the corresponding restrictions of the grading. Then we have a decomposition

$$
{ }^{\rho} K(\mathscr{I}) \cong{ }^{\rho_{r}} K\left(\mathscr{I}^{r e g}\right) \oplus{ }^{\rho_{s}} K\left(\mathscr{I}^{\text {sing }}\right) .
$$

Proposition 2.10. We have

$$
\operatorname{ker}\left(R_{!} \circ \Phi\right)={ }^{\rho_{s}} K\left(\mathscr{I}^{\text {sing }}\right) .
$$

Proof.

2.3.13. The stack $\mathscr{I}$ decomposes into a union of $\hat{W}$-orbits

$$
\mathscr{I} \cong \bigcup_{[\chi] \in X_{1}(\hat{T}) / \hat{W}} \mathscr{I}_{\chi},
$$

where $\mathscr{I}_{\chi}:=[[\chi] / \hat{W}]$. Let $\rho_{\chi}$ be the restriction of $\rho$ to $\mathscr{I}_{\chi}$. Then we have a decomposition

$$
K(\mathscr{I}) \cong \bigoplus_{[\chi] \in X_{1}(\hat{T}) / \hat{W}} \rho_{\chi} K\left(\mathscr{I}_{\chi}\right) .
$$

We consider $\chi \in X_{1}(\hat{T})$. Then we must compute

$$
R_{!} \circ \Phi_{\left.\right|^{\rho_{\chi} K\left(\mathscr{I}_{\chi}\right)}}=R_{!} \circ p_{!} \circ\left(t^{-1}\right)^{*} \circ m^{*} \circ\left(r_{!}\right)_{\left.\right|^{\rho_{\chi} K\left(\mathscr{I}_{\chi}\right)}}^{-1} .
$$

In the present subsection we will show that this composition vanishes for singular characters $\chi$. Regular characters will be discussed later in 2.4.9. 
2.3.14. For the moment, in order to set up some notation, we consider an arbitrary character $\chi \in X_{1}(\hat{T})$. Let $W_{\chi} \subset W$ be the isomorphic image of $\hat{W}_{\chi}$ under $\hat{W} \rightarrow W$. Then we have an equivalence

$$
\mathscr{I}_{\chi} \cong\left[\left(W / W_{\chi}\right) / W\right] \cong\left[* / W_{\chi}\right] .
$$

We let $\tilde{\rho}_{\chi}:\left[\widehat{* / W_{\chi}}\right] \rightarrow\left[* / W_{\chi}\right]$ be the induced grading. It is given by the character $\tilde{\rho}_{\chi}: W_{\chi} \rightarrow \mathbb{Z} / 2 \mathbb{Z}, \tilde{\rho}_{\chi}(w):=\operatorname{det}_{\mathrm{Lie}(T)}(w)$.

2.3.15. To represent $\left(r_{!}\right)_{\left.\right|^{\rho_{\chi}} K\left(\mathscr{I}_{\chi}\right)}^{-1}$ we choose a $\hat{W}$-equivariant section $s$ in

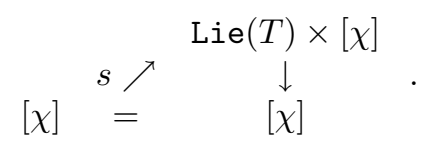

For this we observe that we can choose an element $H \in \operatorname{Lie}(T)$ with $W_{H}=W_{\chi}$. Then we define $s(\hat{w} \chi):=(\hat{w} H, \hat{w} \chi)$.

Let $s: \mathscr{I}_{\chi} \rightarrow \overline{\mathscr{S}}$ denote the induced map of stacks. The normal bundle of $s$ is given by $[\operatorname{Lie}(T) \times[\chi] / \hat{W}] \rightarrow \mathscr{I}_{\chi}$. Therefore $s$ is canonically $-\rho_{\chi}-K$-oriented and we obtain a push-forward $s_{!}:{ }^{\rho_{\chi}} K\left(\mathscr{I}_{\chi}\right) \rightarrow K(\overline{\mathscr{S}})$. We now observe that

$$
\left(r_{!}\right)_{\left.\right|^{\rho_{\chi} K\left(\mathscr{I}_{\chi}\right)}}^{-1}=s !
$$

2.3.16. We have the cartesian diagram

$$
\begin{array}{ccc}
{\left[[\chi] / \check{T} N_{G}(T)\right]} & \stackrel{\tilde{m}}{\rightarrow} & \mathscr{I}_{\chi} \\
\tilde{s} \downarrow & & \downarrow s \\
\mathscr{S} & \stackrel{m}{\rightarrow} & \overline{\mathscr{S}}
\end{array} .
$$

Therefore we can rewrite

$$
R_{!} \circ \Phi_{\left.\right|^{\rho} \chi K\left(\mathscr{I}_{\chi}\right)}=R_{!} \circ p_{!} \circ \tilde{s}_{!} \circ\left(\tilde{s}^{*} t\right)^{-1} \circ \tilde{m}^{*},
$$

The composition $R \circ p \circ \tilde{s}$ is represented by the map

$$
c:\left[\left(\hat{W} / \hat{W}_{\chi}\right) / \check{T} N_{G}(T)\right] \rightarrow \mathscr{M}=\left[F\left(S^{1}\right) / G\left(S^{1}\right)\right] .
$$

On the level of spaces it is given by

$$
\hat{w} \hat{W}_{\chi} \mapsto \hat{w} H .
$$

where $\hat{w} H$ is considered as a constant connection. On the level of groups it is the embedding $\check{T} N_{G}(T) \rightarrow G\left(S^{1}\right)$. Using the equivalences $\mathscr{M} \stackrel{\text { hol }}{\cong}[G / G]$ and

$$
\left[\left(\hat{W} / \hat{W}_{\chi}\right) / \check{T} N_{G}(T)\right] \cong\left[\left(W / W_{\chi}\right) / N_{G}(T)\right] \stackrel{\text { ind }}{\cong}\left[\left(G / N_{G}(T)_{\chi}\right) / G\right]
$$

we have an equivalent representation of $c$ as $G$-equivariant map

$$
c:\left[\left(G / N_{G}(T)_{\chi}\right) / G\right] \rightarrow[G / G]
$$


which is given by $[g] \mapsto \exp (g H)$. Note that this map factors over $G / G_{t}$, so that we have a factorization of $c$ as

$$
\left[\left(G / N_{G}(T)_{\chi}\right) / G\right] \stackrel{a}{\rightarrow}\left[\left(G / G_{t}\right) / G\right] \stackrel{b}{\rightarrow}[G / G] .
$$

The map $a$ is the induction of

$$
a:\left[* / N_{G}(T)_{\chi}\right] \rightarrow\left[* / G_{t}\right] .
$$

2.3.17. From now on until the end of the proof we use the holonomy isomorphism $\mathscr{M} \cong[G / G]$ in order to view $[G / G]$ as the target of $R$. Furthermore we will write $\tau$ for $\mathrm{hol}_{*} \tau$. We can now write

$$
R_{!} \circ \Phi_{\left.\right|^{\rho} \chi K\left(\mathscr{I}_{\chi}\right)}=b_{!} \circ a_{!} \circ\left(\tilde{s}^{*} t\right)^{-1} \circ \tilde{m}^{*} .
$$

Note that $b^{*} \tau$ is already trivial. We choose any trivialization $t^{\prime}: 0 \stackrel{\sim}{\rightarrow} b^{*} \tau$. Then we can further write

$$
R_{!} \circ \Phi_{\left.\right|^{\rho} \chi K\left(\mathscr{I}_{\chi}\right)}=b_{!} \circ\left(t^{\prime,-1}\right)^{*} \circ a_{!} \circ\left(a^{*} t^{\prime}\right)^{*} \circ\left(\tilde{s}^{*} t\right)^{-1} \circ \tilde{m}^{*} .
$$

2.3.18. The embedding $\left[* / N_{G}(T)_{\chi}\right] \rightarrow\left[[\chi] / \check{T} N_{G}(T)\right]$ induced by $* \mapsto \chi$ and the inclusion of groups $N_{G}(T)_{\chi} \rightarrow \breve{T} N_{G}(T)$ is an equivalence of stacks. The automorphism $\left(\tilde{s}^{*} t\right)^{-1} \circ a^{*} t^{\prime}$ of the trivial twist can therefore be considered as a $N_{G}(T)_{\chi}$-equivariant line bundle on $*$. Such a line bundle determines a character $\mu \in X(T)$ which is necessarily $W_{\chi}$-invariant.

2.3.19. If $\chi$ is singular, then $\mu$ is a character of $T$ which is singular (for $G_{t}$ ). In this case the composition

$$
\left(a^{*} t^{\prime}\right)^{*} \circ\left(\tilde{s}^{*} t\right)^{-1} \circ \tilde{m}^{*}:{ }^{\rho_{\chi}} K\left(\mathscr{I}_{\chi}\right) \rightarrow K\left(\left[* / N_{G}(T)_{\chi}\right]\right) \cong R\left(N_{G}(T)_{\chi}\right)
$$

produces a representations of $N_{G}(T)_{\chi}$ on which $T$ acts by a singular characters, and by Borel-Weyl-Bott the induction $a_{!}: K\left(\left[* / N_{G}(T)_{\chi}\right]\right) \rightarrow K\left(\left[* / G_{t}\right]\right)$ vanishes on singular characters. This shows that $R_{!} \circ \Phi$ vanishes on the contributions of singular orbits and

$$
{ }^{\rho_{s}} K\left(\mathscr{I}^{\text {sing }}\right) \subset \operatorname{ker}\left(R_{!} \circ \Phi\right) .
$$

The opposite inclusion $\operatorname{ker}\left(R_{!} \circ \Phi\right) \subset{ }^{\rho_{s}} K\left(\mathscr{I}^{\text {sing }}\right)$ will be shown in 2.4.9.

\subsection{Detection of elements of twisted $K$-theory .}

2.4.1. We start with a twist $\tau: \hat{\mathscr{M}} \rightarrow \mathscr{M}$ which is given by a central $U(1)$ extension $\hat{G}\left(S^{1}\right) \rightarrow G\left(S^{1}\right)$. By restriction (see 2.1.4 for the notation) we obtain a central extension

$$
0 \rightarrow U(1) \rightarrow \check{T} \widehat{T N_{G}(T)} \rightarrow \check{T} N_{G}(T) \rightarrow 0
$$


Since we assume that $\tau$ is admissible this extension is trivial when restricted to $\check{T}$ and $N_{G}(T)$.

2.4.2. Recall (see 2.1.4) that $\check{T}$ acts on $X_{1}(\hat{T})$. We choose a splitting homomorphism $s_{0}: N_{G}(T) \rightarrow \breve{T} \widehat{N_{G}(T)}$ and define a bilinear form

$$
B: \check{T} \otimes T \rightarrow U(1)
$$

by

$$
B(\check{t}, t):=\frac{(\check{t} \chi)\left(s_{0}(t)\right)}{\chi\left(s_{0}(t)\right)} .
$$

It is easy to check that $B$ does not depend on the choice of $\chi \in X_{1}(\hat{T})$ and the split $s_{0}$. Using the form $B$ we can write the cocycle defining the extension

$$
0 \rightarrow U(1) \rightarrow \widehat{\check{T} T} \rightarrow \check{T} T \rightarrow 0
$$

in the form $\omega\left((\check{t}, t),\left(\check{t}^{\prime}, t^{\prime}\right)\right)=B\left(\check{t}, t^{\prime}\right)-B\left(\check{t}^{\prime}, t\right)$. If $s_{1}: \check{T} \rightarrow \widehat{\widetilde{T}}$ is a splitting homomorphism of $\widehat{\widetilde{T}} \rightarrow \check{T}$, then we have

$$
s_{1}(\check{t}) s_{0}(t) s_{1}(\check{t})^{-1}=s_{0}(t) B(\check{t}, t) .
$$

Note that $B$ is $W$-invariant in the sense that $B\left(\check{t}^{w}, t^{w}\right)=B(\check{t}, t)$.

2.4.3. We define a subgroup $F \subset T$ by

$$
F:=\{t \in T \mid B(\check{t}, t)=1 \quad \forall \check{t} \in \check{T}\} \subset T .
$$

Since $B$ is $W$-invariant the action of the Weyl group $W$ on $T$ preserves $F$. Note that $F$ only depends on the central extension $\hat{G}\left(S^{1}\right) \rightarrow G\left(S^{1}\right)$. If the twist $\tau$ is regular, then $B$ is non-degenerated. In this case $F$ is finite.

2.4.4. Let $X(\check{T})$ be the group of characters. We have a map $b: F \backslash T \rightarrow X(\check{T})$ given by $b(F t)(\check{t})=B(\check{t}, F t)^{-1}$.

Lemma 2.15. If $\tau$ is regular, then $b$ is an isomorphism.

Proof. By the definition of $F$ the map $b$ is injective. Regularity of the twist $\tau$ (see 2.1.6) is equivalent to non-degeneracy of $B$. In this case this map $b$ is also surjective.

2.4.5. The restriction of the extension (2.13) to $\check{T} F$ is trivialized by the choices of $s_{0}, s_{1}$ in 2.4.2, i.e. we have a homomorphism $s: \check{T} F \rightarrow \overleftarrow{\overleftarrow{T} F}$ given by $s(\check{t} f):=s_{1}(\check{t}) s_{0}(f)$.

Let $\chi \in X_{1}(\hat{T})$. Then the restriction $\chi_{\mid F}$ is $\check{T}$-invariant. Therefore we get a natural map $s^{*}: X_{1}(\hat{T}) / \check{T} \rightarrow X(F)$ which is $W$-equivariant. 
Lemma 2.16. If $\tau$ is regular, then the map $s^{*}: X_{1}(\hat{T}) / \check{T} \rightarrow X(F)$ is a bijection.

Proof. Surjectivity follows from the following easy assertions. Let $\bar{\mu}$ be a character of $F$. Then there exists a character of $T$ such that $\bar{\mu}=\mu_{\mid F}$. Furthermore, using the split $s_{0}: T \rightarrow \hat{T}$ and $\hat{T} \cong U(1) \times T$ we see that there exists a character $\chi_{\mu, s} \in X_{1}(\hat{T})$ such that $s_{0}^{*} \chi_{\mu, s}=\mu$. It follows that $s^{*} \chi_{\mu, s}=\bar{\mu}$.

Assume now that $s^{*} \chi=s^{*} \chi^{\prime}$. We write $\chi^{\prime}=\chi+\lambda$ for some $\lambda \in X(T)$, where we consider $X(T) \subset X(\hat{T})$ naturally. Then $s^{*} \lambda=0$. Therefore $\lambda$ pulls back from $T / F$. In follows from Lemma 2.15 that there exists $\check{t} \in \check{T}$ such that $\lambda(t)=B(\check{t}, t)$ for all $t \in T$. Therefore, $\check{t} \chi=\chi^{\prime}$.

Let $X^{r e g}(F) \subset X(F)$ be the subset of regular characters, i.e. characters with trivial stabilizer in $W$. The bijection $s^{*}$ restricts to an identification of $W$-sets $s^{*}: X^{r e g}(\hat{T}) / \check{T} \rightarrow X^{r e g}(F)$ and therefore induces a bijection

$$
X^{r e g}(\hat{T}) / \hat{W} \stackrel{\sim}{\rightarrow} X^{r e g}(F) / W .
$$

2.4.6. Let us from now on assume that the twist $\tau$ is regular. We consider the composition of maps

$$
[\operatorname{Lie}(T) / \check{T} F] \stackrel{S}{\rightarrow}\left[\operatorname{Lie}(T) / \check{T} N_{G}(T)\right] \stackrel{R}{\rightarrow} \mathscr{M} .
$$

The twist

$$
S^{*} R^{*} \tau:[\operatorname{Lie}(T) / \widehat{T} F] \rightarrow[\operatorname{Lie}(T) / \check{T} F]
$$

is trivialized by the section $s$ (see 2.4.5). We let $u: 0 \stackrel{\sim}{\rightarrow} S^{*} R^{*} \tau$ denote the corresponding isomorphism. Let $q:[\operatorname{Lie}(T) / \check{T} F] \rightarrow[* / F]$ be induced by the projection to a point. It is representable, proper, and $K$-oriented once we have fixed an orientation of Lie $(T)$. We can now define a map

$$
\Theta: q ! \circ u^{*} \circ S^{*} \circ R^{*}:{ }^{\tau} K(\mathscr{M}) \rightarrow R(F) .
$$

2.4.7. Let $[\chi] \in X_{1}^{r e g}(\hat{T}) / \hat{W}$. We trivialize the twist $\rho_{\chi}$ (see 2.3.13) by choosing an orientation of $\operatorname{Lie}(T)$ and the representative $\chi$ of the class $[\chi]$. In fact this data orients the projection $\operatorname{Lie}(T) \times\{\chi\} \rightarrow\{\chi\}$, and we extend this $\hat{W}$ equivariantly to an orientation of $[\operatorname{Lie}(T) \times[\chi] / \hat{W}] \rightarrow \mathscr{I}_{\chi}$.

We define the homomorphism sign $: \hat{W} \rightarrow\{1,-1\}$ such that $\operatorname{sign}(\hat{w})=$ \pm 1 depending on whether $\hat{w}: \operatorname{Lie}(T) \rightarrow \operatorname{Lie}(T)$ preserves or reverses the orientation.

Then we consider the generator $e_{\chi} \in{ }^{\rho_{\chi}} K\left(\mathscr{I}_{\chi}\right) \cong K(*) \cong \mathbb{Z}$ and set $E_{\chi}:=$ $\left(R_{!} \circ \Phi\right)\left(e_{[\chi]}\right) \in{ }^{\tau} K(\mathscr{M})$. Note that $E_{\hat{w} \chi}=\operatorname{sign}(\hat{w}) E_{\chi}$ for $\hat{w} \in \hat{W}$. In the 
following we will often write the action of Weyl group elements as an exponent, e.g. $\chi^{w}$ means $w \chi$.

Theorem 2.17. We have

$$
\Theta\left(E_{\chi}\right)=\sum_{w \in W} \operatorname{sign}(w)\left(s^{*} \chi\right)^{w} \in R(F) .
$$

Proof. By Proposition 2.8 we have $R^{*} E_{\chi}=\Phi\left(e_{\chi}\right)$. We must compute $\left(q ! \circ u^{*} \circ S^{*}\right)\left(\Phi\left(e_{\chi}\right)\right)$. We have a diagram

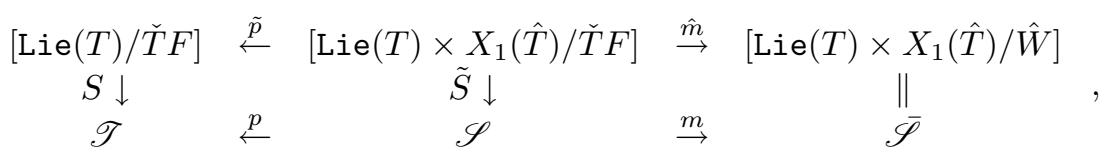

where the left square is cartesian, and the right square commutes. We have

$$
\begin{aligned}
\left(q_{!} \circ u^{*} \circ S^{*}\right)\left(\Phi\left(e_{[\chi]}\right)\right. & \left.=\left(q ! \circ u^{*} \circ S^{*} \circ p_{!} \circ\left(t^{-1}\right)^{*} \circ m^{*} \circ\left(r_{!}\right)^{-1}\right)\left(e_{[\chi]}\right)\right) \\
& =\left(q ! \circ \tilde{p}_{!} \circ\left(\tilde{p}^{*} u\right)^{*} \circ\left(\tilde{t}^{-1}\right)^{*} \circ \hat{m}^{*} \circ\left(r_{!}\right)^{-1}\right)\left(e_{[\chi]}\right) .
\end{aligned}
$$

The trivialization $\tilde{S}^{*} t=: \tilde{t}: 0 \rightarrow \tilde{p}^{*} \circ S^{*} \circ R^{*} \tau$ is given by a $\widehat{T} F$-equivariant $U(1)$-bundle of weight one

$$
\operatorname{Lie}(T) \times X_{1}(\hat{T}) \times U(1) \rightarrow \operatorname{Lie}(T) \times X_{1}(\hat{T}),
$$

where $F$ acts on the fibre over $\operatorname{Lie}(T) \times\{\chi\}$ by $s^{*} \chi:=\chi \circ s: F \rightarrow U(1)$.

A small calculation shows that the trivialization $\tilde{p}^{*} u$ is given by a $\widehat{T F}$ equivariant $U(1)$-bundle of weight one

$$
\operatorname{Lie}(T) \times X_{1}(\hat{T}) \times U(1) \rightarrow \operatorname{Lie}(T) \times X_{1}(T),
$$

where $\widehat{\breve{T} F}$ acts on the $U(1)$-factor via its homomorphism $\widehat{\breve{T} F} \rightarrow U(1)$ induced by the split $s$.

We conclude that

$$
\left(q ! \circ u^{*} \circ S^{*}\right)\left(\Phi\left(e_{\chi}\right)\right)=\sum_{w \in W} \operatorname{sign}(w)\left(s^{*} \chi\right)^{w} \in R(F) .
$$

2.4.8. Let $R(F)$ (sign) $\subset R(F)$ be the subspace of elements satisfying $\lambda^{w}=$ $\operatorname{sign}(w) \lambda$ for all $w \in W$. Then the image of $\Theta$ is contained in $R(F)$ (sign). 
2.4.9. We now finish the proof of Proposition 2.10. After a choice of representatives $\chi \in X_{1}^{r e g}(\hat{T})$ for the equivalence classes $X_{1}^{r e g}(\hat{T}) / \hat{W}$ the elements $e_{\chi}$, $[\chi] \in X_{1}^{r e g}(\hat{T}) / \hat{W}$, form a $\mathbb{Z}$-basis of ${ }^{\rho_{r}} K\left(\mathscr{I}^{r e g}\right)$. By Theorem 2.17 and Lemma 2.16 the composition $\Theta \circ R_{!} \circ \Phi_{\mid \rho_{r} K\left(\mathscr{I}^{r e g}\right)}:{ }^{\rho_{r}} K\left(\mathscr{I}^{r e g}\right) \rightarrow R(F)$ is injective. This implies that $\operatorname{ker}\left(R_{!} \circ \Phi\right) \subset{ }^{\rho_{s}} K\left(\mathscr{I}^{\text {sing }}\right)$. Thus we have finished the proof of Proposition 2.10.

2.4.10. By a combination of Propositions 2.10, 2.8, and 2.7 we see that the elements

$$
E_{\chi}:=R_{!} \circ \Phi\left(e_{\chi}\right), \quad[\chi] \in X_{1}^{r e g}(\hat{T}) / \hat{W}
$$

form a $\mathbb{Z}$-basis of ${ }^{\tau} K(\mathscr{M})$. Up to a sign this basis is natural. The sign depends on the choice of an orientation of $\operatorname{Lie}(T)$. This finishes the proof of Theorem 2.1 .

2.4.11. The group ${ }^{\operatorname{hol}_{*} \tau} K[G / G]$ is a module over $K([* / G]) \cong R(G)$. Indeed, let $p:[G / G] \rightarrow[* / G]$ be the projection. We use the isomorphism $R(G) \cong$ $K([* / G])$ and the $\cup$-product $\cup: K([G / G]) \otimes{ }^{\text {hol }_{*} \tau} K([G / G]) \rightarrow{ }^{\text {hol }_{*} \tau} K([G / G])$. Then the module structure is given by $U \otimes X \mapsto U \bullet X:=p^{*} U \cup X$, where $U \in R(G)$ and $X \in$ hol $_{*} \tau K([G / G])$.

2.4.12. Since ${ }^{\tau} K([G / G])$ is a free $\mathbb{Z}$-module it embeds into its complexification ${ }^{\tau} K([G / G])_{\mathbb{C}}:={ }^{\tau} K([G / G]) \otimes_{\mathbb{Z}} \mathbb{C}$. We let $R(G)_{\mathbb{C}}$ be the complexified group ring of $G$. The action $\bullet$ extends to a linear action

$$
\text { • : } R(G)_{\mathbb{C}} \otimes_{\mathbb{C}}{ }^{\tau} K([G / G])_{\mathbb{C}} \rightarrow{ }^{\tau} K([G / G])_{\mathbb{C}}
$$

Theorem 2.19. The $R(G)_{\mathbb{C}-m o d u l e}{ }^{\tau} K([G / G])_{\mathbb{C}}$ is isomorphic to a quotient of $R(G)_{\mathbb{C}}$.

Proof. Let $\mathbb{C}[F]^{W} \cong \mathbb{C}[F / W]$ denote the algebra of Weyl-invariant $\mathbb{C}$-valued functions on $F$. We have surjective restriction homomorphisms

$$
R(G)_{\mathbb{C}} \rightarrow R(T)_{\mathbb{C}}^{W} \rightarrow \mathbb{C}[F]^{W} \cong \mathbb{C}[F / W] .
$$

Let $F^{\text {reg }} \subset F$ be the subset of elements with trivial stabilizer in $W$. We consider the associated vector bundle $V:=F^{r e g} \times_{W \text {,sign }} \mathbb{C} \rightarrow F^{r e g} / W$. The complexification of $R(F)$ (sign) can be identified with the space $\Gamma(V)$ of sections of $V$ which is a $\mathbb{C}[F / W]$-module. The complexification of $\Theta$ provides an injection

$$
\Theta_{\mathbb{C}}:{ }^{\tau} K([G / G])_{\mathbb{C}} \rightarrow R(F)(\text { sign })_{\mathbb{C}} \stackrel{\sim}{\rightarrow} \Gamma(V)
$$

of $\mathbb{C}$-vector spaces. It follows immediately from the definition of $\Theta$, that this

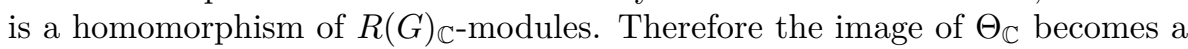


$\mathbb{C}[F / W]$-submodule. Such a submodule is completely determined by its support $S \subset F^{r e g} / W$. We define the ideal $I \subset R(G)_{\mathbb{C}}$ by the sequence

$$
0 \rightarrow I \rightarrow R(G)_{\mathbb{C}} \rightarrow \mathbb{C}[F / W] / \mathbb{C}[S] \rightarrow 0
$$

Then have

$$
{ }^{\tau} K([G / G])_{\mathbb{C}} \cong R(G)_{\mathbb{C}} / I
$$

\subsection{The identity.}

2.5.1. We consider the map of stacks $e:[* / G] \rightarrow[G / G]$ which on the level of spaces is given by the identity element of $G$. Let $\tau$ be a twist of $\mathscr{M}$ and $\mathrm{hol}_{*} \tau$ be the corresponding twist of $[G / G]$. In the present subsection in order to simplify the notation we will denote this twist simply by $\tau$.

We shall assume that $e^{*} \tau$ is trivial and fix a trivialization $t: e^{*} \tau \stackrel{\sim}{\rightarrow} 0$. Note that we can write $e^{*} \tau:[* / \hat{G}] \rightarrow[* / G]$ for a $U(1)$-central extension $\hat{G} \rightarrow G$ of $G$. The datum of a trivialization $t$ is equivalent to a split $\varphi: G \rightarrow \hat{G}$.

We assume that the twist $\sigma(G)$ (see 2.2.14) is trivialized. The map $e$ is $K$-oriented once we have chosen an orientation of Lie $(G)$ (see 2.2.12). Then we define the element

$$
E:=e_{!} t^{*}(1) \in{ }^{\tau} K([G / G]) .
$$

The goal of the present subsection is an explicit calculation of $E$. We obtain a formula for $E$ by calculating $\Theta(E) \in R(F)$, where $\Theta$ and $F$ are as in Theorem 2.17 .

The element $E$ will give the identity of the ring structure on ${ }^{\tau} K([G / G])$ discussed in Subsection 3.3.

2.5.2. We fix a positive root system $\Delta \subset X(T)$ of $(\operatorname{Lie}(G), \operatorname{Lie}(T))$ and let $\rho:=\frac{1}{2} \sum_{\alpha \in \Delta} \alpha$. The restriction of the split $\varphi$ to the torus $T$ induces a bijection $\varphi^{*}: X_{1}(\hat{T}) \rightarrow X(T)$. We define $\chi_{\varphi, \rho} \in X_{1}(\hat{T})$ by the condition $\varphi^{*} \chi_{\varphi, \rho}=\rho$. According to Theorem 2.1 this character determines a basis element $E_{\chi_{\varphi, \rho}} \in$ ${ }^{\tau} K([G / G])$.

Note that the orientation of $\operatorname{Lie}(G)$ induces an orientation of $\operatorname{Lie}(T)$, since $\operatorname{Lie}(G) \cong \operatorname{Lie}(T) \oplus \operatorname{Lie}(G) / \operatorname{Lie}(T)$, and the choice of the positive root system $\Delta$ fixes a complex structure (and hence an orientation) on $\operatorname{Lie}(G) / \operatorname{Lie}(T)$. This fixes the sign of the basis element.

Theorem 2.20. We have $E=E_{\chi \varphi, \rho}$. 
2.5.3. The remainder of the present subsection is devoted to the proof of this formula. The character $\rho$ of $T$ determines an associated $G$-equivariant line bundle $G \times_{T, \rho} \mathbb{C} \rightarrow G / T$. Let $x_{\rho} \in K([(G / T) / G])$ denote the $K$-theory element represented by this bundle. Let $b:[(G / T) / G] \rightarrow[* / G]$ be induced by the projection $G / T \rightarrow *$. The choice of $\Delta$ induces a $G$-equivariant complex structure on the tangential bundle $T(G / T)$. This gives the $K$-orientation of $b$. The following is well-known in representation theory and a consequence of the Borel-Weyl-Bott theorem.

Lemma 2.21. We have

$$
b_{!} x_{\rho}=1 \in K([* / G]) \cong R(G) .
$$

2.5.4. We consider now the diagram

$$
\begin{array}{ccc}
{[* / G]} & \stackrel{e}{\rightarrow} & {[G / G]} \\
b \uparrow & & R \uparrow \\
{[(G / T) / G]} & \stackrel{c}{\rightarrow} & {\left[T / N_{G}(T)\right]}
\end{array}
$$

The lower horizontal map is given by $c:[(G / T) / G] \stackrel{\sim}{\rightarrow}[* / T] \stackrel{\tilde{c}}{\rightarrow}\left[T / N_{G}(T)\right]$, where $\tilde{c}$ is given by the identity of $T$ on the level of spaces, and by the embedding $T \mapsto N_{G}(T)$ on the level of groups. We see that

$$
e_{!} t^{*}(1)=e_{!} t^{*}\left(b_{!} x_{\rho}\right)=e_{!} b_{!} b^{*}(t)^{*}\left(x_{\rho}\right)=R_{!} c_{!} b^{*}(t)^{*}\left(x_{\rho}\right) .
$$

Under $[(G / T) / G] \stackrel{\sim}{\rightarrow}[* / T]$ the element $x_{\rho}$ corresponds to $\rho \in R(T) \cong K([* / T])$. The trivialization $t$ induces some trivialization $v: \tilde{c}^{*} R^{*} \tau \stackrel{\sim}{\rightarrow} 0$. Actually $\tilde{c}^{*} R^{*} \tau$ : $[* / \hat{T}] \rightarrow[* / T]$, and $v$ is induced by a split $\varphi: T \rightarrow \hat{T}$. At the moment we can take an arbitrary split, but later (see 2.5.9) it will be important that $\varphi$ is the restriction of $\varphi: G \rightarrow \hat{G}$ (see 2.5.1). Using the notation of 2.4.6 we see that we must calculate

$$
q ! \circ u^{*} \circ S^{*} \circ \tilde{c}_{!} \circ v^{*}(\rho) \in R(F) .
$$

2.5.5. In the next paragraph 2.5.6 we perform a longer calculation of certain pull-back diagrams. Since we will use this result in later subsections with different input we will state it in a general form. For the purpose of the present subsection the symbols have the following meaning.

(1) $X:=\operatorname{Lie}(T), Q:=\check{T} N_{G}(T)$

(2) $Z:=*, L:=T$

(3) $Y:=\operatorname{Lie}(T), H:=\check{T} F$

We have natural homomorphisms $L \rightarrow Q \leftarrow H$. Furthermore, we have a central extension $\hat{Q} \rightarrow Q$ given by $\check{T} \widehat{N_{G}(T)} \rightarrow \check{T} N_{G}(T)$ such that its restrictions 
$\hat{H} \rightarrow H$ and $\hat{L} \rightarrow L$ are trivialized by sections $s$ and $\varphi$. We consider the pull-back diagram

$$
\begin{array}{ccccc}
P & \stackrel{a}{\rightarrow} & {[Y / H]} & \stackrel{q}{\rightarrow} & {[* / H]} \\
d \downarrow & & S \downarrow \\
{[Z / L]} & \stackrel{\tilde{c}}{\rightarrow} & {[X / Q]} & &
\end{array}
$$

Over the left upper corner $P$ we have two trivializations of the twist $a^{*} S^{*} R^{*} \tau \cong$ $d^{*} \tilde{c}^{*} R^{*} \tau$, namely $a^{*} s$ and $d^{*} \varphi$. The composition $U:=a^{*} u \circ d^{*} v$ is an automorphism of the trivial twist and therefore a line bundle over $P$. Using $S^{*} \circ \tilde{c}_{!}=$ $a_{!} \circ d^{*}$ we see that we must calculate

$$
q_{!} \circ a_{!}\left([U] \cup\left(d^{*} \rho\right)\right) \in R(F),
$$

where $[U] \in K(P)$ is the element represented by $U$.

2.5.6. We consider the following diagram of stacks

$$
[Z / L] \rightarrow[X / Q] \leftarrow[Y / H]
$$

We make the pull-back $P$ explicit and get

$$
\begin{array}{ccc}
{\left[(Z \times Q) \times_{X}(Y \times Q) / L \times H \times Q\right]} & \rightarrow & {[Y / H]} \\
\downarrow & & \downarrow \\
{[Z / L]} & \rightarrow & {[X / Q]}
\end{array}
$$

Here the $(Z \times Q) \times_{X}(Y \times Q) \subset Z \times Q \times Y \times Q$ is defined by the equation $g_{1} z=g_{2} y$, where $\left(z, g_{1}, y, g_{2}\right) \in Z \times Q \times Y \times Q$. The action is given by

$$
(l, h, g)\left(z, g_{1}, y, g_{2}\right)=\left(l z, g g_{1} l^{-1}, h z, g g_{2} h^{-1}\right) .
$$

The left vertical map is given by $\left(z, g_{1}, y, g_{2}\right) \mapsto z$ on the level of spaces, and by $(l, h, g) \mapsto l$ on the level of groups. The upper horizontal map is similar.

We have further pull-backs

$$
\begin{array}{ccccccc}
{[Z \times Q / L \times \hat{Q}]} & \rightarrow & {[X / \hat{Q}]} & & {[Y \times Q / H \times \hat{Q}]} & \rightarrow & {[X / \hat{Q}]} \\
\downarrow & & \downarrow & & \downarrow & & \downarrow \\
{[Z / L]} & \rightarrow & {[X / Q]} & & {[Y / H]} & \rightarrow & {[X / Q]}
\end{array}
$$

We give more details for the left square. The action is given by

$$
(l, \hat{g})(z, g)=\left(l z, \hat{g} g l^{-1}\right) .
$$

The left vertical map is given by $(z, g) \mapsto z$ on the level of spaces, and by $(l, \hat{g}) \mapsto l$ on the level of groups. The upper horizontal map is given by $(z, g) \mapsto$ 
$g z$ on the level of spaces, and by $(l, \hat{g}) \mapsto \hat{g}$ on the level of groups. We pull-back further:

$$
\begin{array}{ccc}
{\left[(Z \times Q) \times \times_{X}(Y \times Q) / L \times H \times \hat{Q}\right]} & \rightarrow & {[Z \times Q / L \times \hat{Q}]} \\
\downarrow & \downarrow & \downarrow \\
{\left[(Z \times Q) \times \times_{X}(Y \times Q) / L \times H \times Q\right]} & \rightarrow & {[Z / L]}
\end{array}
$$

The split of $\varphi: \hat{L} \rightarrow L$ gives rise to an action of $L$ on $\hat{Q}$. We form the $U(1)$-bundle of weight one

$$
[Z \times \hat{Q} / L \times \hat{Q}] \rightarrow[Z \times Q / L \times \hat{Q}]
$$

where the action is given by

$$
(l, \hat{g})\left(z, \hat{g}_{1}\right)=\left(l z, \hat{g} \hat{g}_{1} \varphi\left(l^{-1}\right)\right) .
$$

We consider the pull-back

$$
\begin{aligned}
& \mathscr{L}:=\left[(Z \times \hat{Q}) \times_{X}(Y \times Q) / L \times H \times \hat{Q}\right] \rightarrow[Z \times \hat{Q} / L \times \hat{Q}] \\
& {\left[(Z \times Q) \times_{X}(Y \times Q) / L \times H \times \hat{Q}\right] \rightarrow[Z \times Q / L \times \hat{Q}]}
\end{aligned}
$$

with the action

$$
(l, h, \hat{g})\left(z, \hat{g}_{1}, y, g_{2}\right)=\left(l z, \hat{g} \hat{g}_{1} \varphi\left(l^{-1}\right), \hat{g} g_{2} h^{-1}\right) .
$$

A similar construction with $[Y / H]$ gives the $U(1)$-bundle

$$
\mathscr{H}:=\left[(Z \times Q) \times_{X}(Y \times \hat{Q}) / L \times H \times \hat{Q}\right] \rightarrow\left[(Z \times Q) \times_{X}(Y \times Q) / L \times H \times \hat{Q}\right]
$$

with the action

$$
(l, h, \hat{g})\left(z, g_{1}, y, \hat{g}_{2}\right)=\left(l z, \hat{g} g_{1} l^{-1}, \hat{g} \hat{g}_{2} s\left(h^{-1}\right)\right) .
$$

The bundle of fibrewise $U(1)$-isomorphisms

$$
\operatorname{Hom}(\mathscr{L}, \mathscr{H}) \rightarrow\left[(Z \times Q) \times_{X}(Y \times Q) / L \times H \times \hat{Q}\right]
$$

admits an action of $L \times H \times Q$ in a natural way.

We simplify the description of the bundle

$$
[\operatorname{Hom}(\mathscr{L}, \mathscr{H}) / L \times H \times Q] \rightarrow\left[(Z \times Q) \times_{X}(Y \times Q) / L \times H \times Q\right] .
$$

We first consider the special case

$$
[\operatorname{Hom}(\hat{Q} \times Q, Q \times \hat{Q}) / Q] \rightarrow[Q \times Q / Q]
$$

where $Q$ acts diagonally. This bundle is equivalent to

$$
\operatorname{Hom}(\hat{Q}, Q \times U(1)) \rightarrow Q .
$$


In our case the bundles come with an action of $L \times H$. In the simplified picture the action is given by

$$
(l, h)(\psi)(\hat{g})=\psi\left(s(h) \hat{g} \varphi\left(l^{-1}\right)\right)
$$

over $(l, h)(g)=h g l^{-1}$ on $Q$. We obtain the equivalent description in the form

$$
\left[(Z \times \operatorname{Hom}(\hat{Q}, Q \times U(1))) \times_{X} Y / L \times H\right] \rightarrow\left[(Z \times Q) \times_{X} Y / L \times H\right],
$$

where the action is given by

$$
(l, h)(z, \varphi, y)=(l z,(l, h)(\varphi), h y)
$$

over $(l, h)(z, g, y)=\left(l z, h g l^{-1}, h y\right)$, and the subscript $\times_{X}$ stands for the relation $g z=y$.

2.5.7. We use the calculation 2.5.6 in order to compute $U$. The result is

$$
\begin{aligned}
& \left.U \cong\left[\check{T} \widehat{T N_{G}(T)}\right)^{*} \times \operatorname{Lie}(T) \operatorname{Lie}(T) / T \times \check{T} F\right] \\
& P \cong\left[\check{T} N_{G}(T) \times_{\mathrm{Lie}(T)} \operatorname{Lie}(T) / T \times \check{T} F\right]
\end{aligned}
$$

The condition $\times_{\operatorname{Lie}(T)}$ stands for $\hat{n} 1=l$, where $\left.(n, l) \in \widetilde{T} \widehat{T N_{G}(T)}\right)^{*} \times \operatorname{Lie}(T)$. We describe the action in the case of $U$, where it is given by

$$
(t, \check{t} f)(\hat{n}, l)=\left(s(\check{t} f) \hat{n} \varphi\left(t^{-1}\right), \check{t} l\right) .
$$

Using the condition we can simplify the description of $U$ and $P$ to

$$
\begin{aligned}
& \left.U \cong\left[\overleftarrow{T N_{G}(T)}\right)^{*} / T \times \check{T} F\right] \\
& P \cong\left[\check{T} N_{G}(T) / T \times \check{T} F\right]
\end{aligned}
$$

If we tensorize $L$ with $d^{*} \rho$, then we get $\left.U(\rho):=\left[\check{T} \widehat{N_{G}(T)}\right) / T \times \check{T} F\right]$, where the action is now given by

$$
(t, \check{t} f)(\hat{n})=s(\check{t} f) \hat{n} \varphi\left(t^{-1}\right) \rho(t) .
$$

\subsubsection{We write}

$$
P:=\sqcup_{w \in W}[\check{T} T w / T \times \check{T} F] .
$$

For $w \in W$ let $b_{w}:[\check{T} T w / T \times \check{T} F] \rightarrow[* / F]$ be the restriction of $q \circ a$ to the corresponding component. Then we must calculate $\left(b_{w}\right)_{!}\left[U_{w}(\rho)\right] \in R(F)$, where $U_{w}(\rho)$ is the restriction of $U(\rho)$ to $\check{T} T w$. The inclusion $[* / F] \rightarrow[\check{T} T w / T \times \check{T} F]$ given by $* \mapsto w$ on the level of spaces, and by $f \mapsto\left(f^{w^{-1}}, f\right)$ on the level of groups is an isomorphism of stacks. Let $\omega(f):=s(f) \varphi\left(f^{-1}\right)$. Then the restriction of $U_{w}(\rho)$ to $[* / F]$ is the character $\rho\left(f^{w^{-1}}\right) \omega(f)$. We obtain

$$
\Theta(E)=\sum_{w \in W} \operatorname{sign}(w) \rho^{w} \omega
$$


where the sign is obtained by determining the orientation of $b_{w}$ for all $w \in W$ back-tracing the definitions.

2.5.9. Recall that $s_{\mid F}: F \rightarrow \widehat{T T}$ comes as a restriction of a split $s_{0}: T \rightarrow \hat{T}$. For the moment we can choose $s_{0}:=\varphi$. Then we have $\omega=0$ and therefore $\Theta(E)=\sum_{w \in W} \operatorname{sign}(w)\left(s^{*} \chi_{\varphi, \rho}\right)^{w}$. This implies by Theorem 2.17 that $E=E_{\chi \varphi, \rho}$.

\subsection{The anti-diagonal.}

2.6.1. In the present subsection we again assume that the twist $\sigma(G)$ (see 2.2.14) is trivial. We consider the homomorphism

$$
G \rightarrow G \times G, \quad g \mapsto(g, g)
$$

and the $G$-equivariant map

$$
G \mapsto G \times G, \quad g \mapsto\left(g, g^{-1}\right),
$$

where $G$ acts on $G$ and $G \times G$ acts on $G \times G$ by conjugation. In this way we arrive at a map of stacks

$$
\delta:[G / G] \rightarrow[G \times G / G \times G]
$$

which we call the anti-diagonal map.

2.6.2. Let $\tau$ be a twist of $\mathscr{M}$. We assume that $\tau$ is regular, admissible (2.1.6), and odd (2.1.9). The twist $\tau$ induces a twist hol ${ }_{*} \tau$ of $[G / G]$. In the present subsection we will simplify the notation and write $\tau$ for this twist. Let

$$
\operatorname{pr}_{i}:[G \times G / G \times G] \rightarrow[G / G]
$$

denote the projections. We obtain a regular and admissible twist $\sigma:=\operatorname{pr}_{1}^{*} \tau+$ $\operatorname{pr}_{2}^{*} \tau$ of $[G \times G / G \times G]$. It is given by the central extension $\left(G \widehat{\times G)(} S^{1}\right) \rightarrow$ $(G \times G)\left(S^{1}\right)$, where $\left(G \widehat{\times G)}\left(S^{1}\right):=\hat{G}\left(S^{1}\right) \times \hat{G}\left(S^{1}\right) / U(1)\right.$, and the quotient is by the diagonal action.

2.6.3. Note that $\mathrm{pr}_{1} \circ \delta=\mathrm{id}$ and $\mathrm{pr}_{2} \circ \delta=I$ (see 2.1.9). Since $\tau$ is odd, it follows that $\delta^{*} \sigma \cong \tau+I^{*} \tau \cong 0$. We choose a trivialization $t: \delta^{*} \sigma \stackrel{\sim}{\rightarrow} 0$. Note that $\delta$ is representable and proper. We consider the composition

$$
[G \times G / G \times G] \cong[G / G] \stackrel{\delta}{\rightarrow}[G \times G / G \times G] \rightarrow[* / G \times G] .
$$

Here the action of $G \times G$ on $G \times G$ in the left $[G \times G / G \times G]$ is given by $\left(g_{1}, g_{2}\right)\left(h_{1}, h_{2}\right)=\left(g_{1} h_{1} g_{1}^{-1}, g_{2} h_{2}\right)$. This composition is equivalent to the product of $[G / G] \rightarrow[* / G]$ and $* \rightarrow[* / G]$. By 2.2.12 and 2.2.13 and the assumption that $\sigma(G)=0$ these maps are $K$-oriented if we choose an orientation of $\operatorname{Lie}(G)$. 
Then by 2.2.8 also $\delta$ is $K$-oriented. Note that the orientation of $\delta$ is even independent of the choice of the orientation of Lie $(G)$.

We can now define

$$
D:=\delta ! t^{*}(1) \in{ }^{\sigma} K([G \times G / G \times G]) .
$$

The goal of the present subsection is an explicit calculation of $D$.

2.6.4. The class $D$ depends on the choice of the trivialization $t$. In the following paragraph we will define a character $\omega \in X(T)$ which encodes the choice of $t$.

We consider the diagram

$$
\begin{array}{ccc}
{\left[F\left(S^{1}\right) / G\left(S^{1}\right)\right]} & \stackrel{\tilde{\delta}}{\rightarrow} & {\left[F\left(S^{1}\right) \times F\left(S^{1}\right) / G\left(S^{1}\right) \times G\left(S^{1}\right)\right]} \\
\cong \downarrow & \stackrel{\cong}{ } \cong & {[G \times G / G \times G]}
\end{array}
$$

where the vertical equivalences are given by the holonomy maps. The pull-back of $\sigma$ to the right upper corner is given by

$$
\left[F\left(S^{1}\right) \times F\left(S^{1}\right) / G\left(S^{1}\right) \times G\left(S^{1}\right)\right] \rightarrow\left[F\left(S^{1}\right) \times F\left(S^{1}\right) / G\left(S^{1}\right) \times G\left(S^{1}\right)\right],
$$

where $G\left(S^{1}\right) \times G\left(S^{1}\right) \cong \hat{G}\left(S^{1}\right) \times \hat{G}\left(S^{1}\right) / U(1)$ (diagonal action), and $\hat{G}\left(S^{1}\right) \rightarrow$ $G\left(S^{1}\right)$ defines $\tau$.

Let $i: S^{1} \rightarrow S^{1}$ be the inversion map. The map $\tilde{\delta}$ is given by $A \mapsto\left(A, i^{*} A\right)$ and $g \mapsto\left(g, i^{*} g\right)$ in the level of spaces and groups.

The lift of the pull-back $\delta^{*} \sigma$ to the left upper corner is represented by the central extension ${\widehat{G\left(S^{1}\right)}}^{d} \rightarrow G\left(S^{1}\right)$, which is obtained as the restriction of $G\left(S^{1} \widehat{) \times G}\left(S^{1}\right) \rightarrow G\left(S^{1}\right) \times G\left(S^{1}\right)\right.$ via the embedding (id, $\left.i^{*}\right): G\left(S^{1}\right) \rightarrow$ $G\left(S^{1}\right) \times G\left(S^{1}\right)$. The trivialization $t$ is now given by a split $\varphi: G\left(S^{1}\right) \rightarrow{\widehat{G\left(S^{1}\right)}}^{d}$.

Let $s_{0}: T \rightarrow \hat{T}$ denote the split which was chosen in 2.4.2. Then we define another split $s_{0}^{\prime}: T \rightarrow \hat{T}$ by the condition that $\varphi(t)=\left[\left(s_{0}(t), s_{0}(t)^{\prime}\right)\right]$, where we consider $T \subset G\left(S^{1}\right), \hat{T} \subset \hat{G}\left(S^{1}\right)$, and the bracket on the right-hand side denotes the class in $\hat{T} \times_{T} \hat{T} / U(1) \subset{\widehat{G\left(S^{1}\right)}}^{d}$.

We define $\omega \in X(T)$ by the condition that $s_{0}^{\prime}(t)=\omega(t) s_{0}(t), t \in T$.

2.6.5. Let $\widehat{T \times T} \rightarrow T \times T$ be the central extension obtained by the restriction of $\left(G \times \widehat{\times G)(} S^{1}\right)$ to $T \times T \subset(G \times G)\left(S^{1}\right)$, i.e $\widehat{T \times T}=\hat{T} \times \hat{T} / U(1)$ (diagonal action). We define a map

$$
\times: X_{1}(\hat{T}) \times X_{1}(\hat{T}) \rightarrow X_{1}(\widehat{T \times T})
$$

by $\left(\chi \times \chi^{\prime}\right)\left(\left[\hat{t}, \hat{t}^{\prime}\right]\right)=\chi(\hat{t}) \chi^{\prime}\left(\hat{t}^{\prime}\right)^{-1}$. This map is a bijection. 
2.6.6. The affine Weyl group of $G \times G$ is isomorphic to $\hat{W} \times \hat{W}$. The product $\times$ is equivariant in the sense that $\hat{w} \chi \times \tilde{\hat{w}}^{\prime} \chi^{\prime}=\left(\hat{w}, \hat{w}^{\prime}\right)\left(\chi \times \chi^{\prime}\right)$, where $\tilde{\ldots}: \hat{W} \rightarrow$ $\hat{W}$ is the automorphism $\widetilde{\tilde{t} w}:=\check{t}^{-1} w$. In particular, the product $\times$ identifies the subset $X_{1}^{r e g}(\hat{T}) \times X_{1}^{r e g}(\hat{T})$ with $X_{1}^{r e g}(\widehat{T \times T})$. Furthermore we see that it descends to a map of orbits

$$
\overline{\times}: X_{1}(\hat{T}) / \hat{W} \times X_{1}(\hat{T}) / \hat{W} \stackrel{\sim}{\rightarrow} X_{1}(\widehat{T \times T}) / \hat{W} \times \hat{W} .
$$

2.6.7. We apply Theorem 2.1 to the group $G \times G$. It provides basis elements $E_{\kappa} \in{ }^{\sigma} K([G \times G / G \times G])$ labeled by $[\kappa] \in X_{1}^{r e g}(\widehat{T \times T}) /(\hat{W} \times \hat{W})$. The orientation of $\operatorname{Lie}(T \times T) \cong \operatorname{Lie}(T) \oplus \operatorname{Lie}(T)$ induced by a choice of an orientation of Lie $(T)$ is independent of this choice. This fixes the signs of the basis elements.

Theorem 2.23. We have

$$
D= \pm \sum_{[\chi] \in X_{1}^{r e g}(\hat{T}) / \hat{W}} E_{\chi \times(\chi \omega)}
$$

We will explain the origin of the sign during the proof 2.6.15. The idea of the proof is to apply 2.17 .

2.6.8. We consider the cartesian diagram

$$
\begin{array}{ccc}
{\left[T / N_{G}(T)\right]} & \stackrel{j}{\rightarrow} & {\left[T \times T / N_{G}(T) \times N_{G}(T)\right]} \\
R \downarrow & & R \times R \downarrow \\
{[G / G]} & \stackrel{\delta}{\rightarrow} & {[G \times G / G \times G]}
\end{array},
$$

where $j$ is given by the anti diagonal $t \mapsto\left(t, t^{-1}\right)$ on the level of spaces, and by the diagonal $t \mapsto(t, t)$ on the level of groups. The $K$-orientation of $\delta$ induces a $K$-orientation of $j$. We now observe that $1=R_{!} R^{*}(1)=R_{!}(1)$. Therefore we have $D=(R \times R) ! j_{!} R^{*}(t)^{*}(1)$.

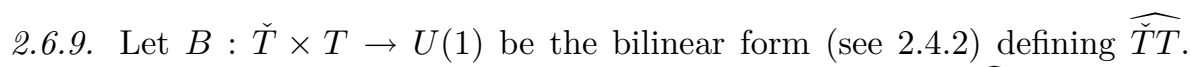
Then the form $\tilde{B}:(\check{T} \times \check{T}) \times(T \times T) \rightarrow U(1)$ which defines $\check{T} \bar{T} \times \check{T} T$ is given by

$$
\tilde{B}\left(\left(\check{t}, \check{t}^{\prime}\right)\left(t, t^{\prime}\right)\right)=B(\check{t}, t) B\left(\check{t}^{\prime}, t^{\prime}\right)^{-1} .
$$

Let $F \subset T$ be the subgroup introduced in 2.4.3. Then we must consider $F \times F \subset T \times T$, correspondingly. 
2.6.10. We now consider the diagram

$$
\begin{array}{ccc}
P & \stackrel{\alpha}{\rightarrow} & {\left[T / N_{G}(T)\right]} \\
p \downarrow & & j \downarrow \\
{[T \times T / F \times F]} & \stackrel{S}{\rightarrow} & {\left[T \times T / N_{G}(T) \times N_{G}(T)\right],} \\
q \downarrow & & \\
{[* / F \times F]} & &
\end{array}
$$

where $P$ is the pull-back. We choose a trivialization $u: 0 \rightarrow S^{*}(R \times R)^{*} \sigma$. Then the injection $\Theta$ (Theorem 2.17) is given by

$$
\Theta=q ! u^{*} S^{*}(R \times R)^{*}:{ }^{\sigma} K([G \times G / G \times G]) \rightarrow R(F \times F) .
$$

Therefore we must calculate

$$
\Theta(D)=q_{!} u^{*} S^{*} j_{!} R^{*}(t)(1)=q_{!} p_{!}(U),
$$

where $U \rightarrow P$ is the line bundle which corresponds to the automorphism $\alpha^{*} R^{*}(t) \circ p^{*} u$ of the trivial twist. Note that the $K$-orientation of $p$ is induced from the $K$-orientation of $j$, and the $K$-orientation of $q$ is given by the orientation of Lie $(T \times T)$.

2.6.11. We write the pull-back diagram (2.24) in the following equivalent form

$$
\begin{array}{ccc}
P & \stackrel{\alpha}{\rightarrow} & {\left[\operatorname{Lie}(T) / \check{T} N_{G}(T)\right]} \\
p \downarrow & & d \downarrow \\
{[\operatorname{Lie}(T) \times \operatorname{Lie}(T) / \check{T} F \times \check{T} F]} & \stackrel{\beta}{\rightarrow} & {\left[\operatorname{Lie}(T) \times \operatorname{Lie}(T) / \check{T} N_{G}(T) \times \check{T} N_{G}(T)\right]} \\
q \downarrow & & \\
{[* / F \times F]} & &
\end{array}
$$

The map $d$ is given by $d(l):=\left(l, l^{-1}\right)$ on the level of spaces, and $d(m):=(m, \tilde{m})$ on the level of groups, where $m \mapsto \tilde{m}$ is the automorphism $\check{T} N_{G}(T) \rightarrow \check{T} N_{G}(T)$ given by $\widetilde{t} u=\check{t}^{-1} u$.

2.6.12. The twist $(R \times R)^{*} \sigma$ is given by the central extension

$$
\check{T} N_{G}(T) \times \check{T} N_{G}(T) \rightarrow \check{T} N_{G}(T) \times \check{T} N_{G}(T) .
$$

By restriction via $d$ we obtain a central extension

$$
\widehat{T}_{N_{G}(T)}^{d} \rightarrow \check{T} N_{G}(T) .
$$

The restriction of the split $\varphi: G\left(S^{1}\right) \rightarrow{\widehat{G\left(S^{1}\right)}}^{d}$ (see 2.6.4) induces a split $\varphi: \check{T} N_{G}(T) \rightarrow \widetilde{T} \widehat{N_{G}(T)}{ }^{d} \subset \check{T} N_{G}\left(\widehat{T) \times \check{T}} N_{G}(T)\right.$. 


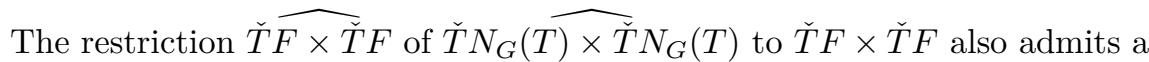

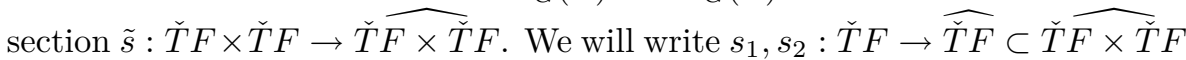
for the restrictions of $\tilde{s}$ to the first and the second factors. We can and will assume that $\left[s_{1}(t), s_{2}(t)\right]=\left[s_{0}(t), s_{0}^{\prime}(t)\right], t \in T$.

2.6.13. In order to calculate the bundle $U \rightarrow P$ explicitly we will employ the general calculation 2.5.6. The symbols in 2.5.6 now have the following meanings.

(1) $X:=\operatorname{Lie}(T) \times \operatorname{Lie}(T), Q:=\check{T} N_{G}(T) \times \check{T} N_{G}(T)$

(2) $Y:=\operatorname{Lie}(T) \times \operatorname{Lie}(T), H:=\check{T} F \times \check{T} F$

(3) $Z:=\operatorname{Lie}(T), L:=\check{T} N_{G}(T)$.

We observe that the restriction $\hat{H} \rightarrow H$ is trivialized by a section $\tilde{s}: H \rightarrow \hat{H}$, and that we also have a section $\varphi: L \rightarrow \hat{L}$.

2.6.14. The calculation of 2.5.6 gives now the following description of the line bundle $U \rightarrow P$.

$\left.U \cong\left[\operatorname{Lie}(T) \times\left(\check{T} N_{G}(T) \times \check{T} N_{G}(T)\right)^{*} \times_{\operatorname{Lie}(T) \times \operatorname{Lie}(T)} \operatorname{Lie}(T) \times \operatorname{Lie}(T)\right] / \check{T} N_{G}(T) \times \check{T} F \times \check{T} F\right]$

$\left.P \cong\left[\operatorname{Lie}(T) \times \check{T} N_{G}(T) \times \check{T} N_{G}(T) \times_{\operatorname{Lie}(T) \times \operatorname{Lie}(T)} \operatorname{Lie}(T) \times \operatorname{Lie}(T)\right] / \check{T} N_{G}(T) \times \check{T} F \times \check{T} F\right]$.

Evaluating the condition $\times_{\operatorname{Lie}(T) \times \operatorname{Lie}(T)}$ we can simplify this description to

$$
\begin{aligned}
U \cong\left[\operatorname{Lie}(T) \times\left(\check{T} N_{G}\left(\widehat{T) \times \check{T}} N_{G}(T)\right)^{*}\right] / \check{T} N_{G}(T) \times \check{T} F \times \check{T} F\right] \\
\left.P \cong\left[\operatorname{Lie}(T) \times \check{T} N_{G}(T) \times \check{T} N_{G}(T)\right] / \check{T} N_{G}(T) \times \check{T} F \times \check{T} F\right] .
\end{aligned}
$$

Next we restrict to the section

$$
\operatorname{Lie}(T) \times\{1\} \times \check{T} N_{G}(T) \subset \operatorname{Lie}(T) \times \check{T} N_{G}(T) \times \check{T} N_{G}(T) .
$$

We get

$$
\begin{aligned}
& \left.U \cong\left[\operatorname{Lie}(T) \times\left(\check{\left(\widetilde{T N_{G}(T}\right)^{r}}\right)^{*}\right] / \check{T} F \times \check{T} F\right] \\
& \left.P \cong\left[\operatorname{Lie}(T) \times \check{T} N_{G}(T)\right] / \check{T} F \times \check{T} F\right] \text {, }
\end{aligned}
$$

where $\left.\check{T N_{G}(T}\right)^{r} \subset \check{T} N_{G}\left(\widehat{T) \times \check{T}} N_{G}(T)\right.$ is the preimage of $\{1\} \times \check{T} N_{G}(T)$. We describe the action for $U$ :

$$
(a, b)(l, \hat{m})=\left(a l, s_{2}(\tilde{b}) \hat{m} s_{1}(a) \varphi\left(a^{-1}\right)\right),
$$

where we consider $s_{1}(a) \varphi\left(a^{-1}\right) \in \check{T}{\widehat{N_{G}(T)}}^{r}$ in the natural way.

We now write

$$
\check{T} N_{G}(T)=\sqcup_{w \in W} \check{T} T w
$$

We further restrict to the section

$$
\sqcup_{w \in W} T w \subset \sqcup_{w \in W} \check{T} T w
$$


We get

$$
\begin{aligned}
& \left.U \cong\left[\operatorname{Lie}(T) \times \sqcup_{w \in W}\left(\hat{T}^{*} w\right)^{r}\right] / \check{T} \times F \times F\right] \\
& \left.P \cong\left[\operatorname{Lie}(T) \times \sqcup_{w \in W} T w\right] / \check{T} \times F \times F\right],
\end{aligned}
$$

where $\left.\left(\hat{T}^{*} w\right)^{r} \subset \check{T} \widehat{N}_{N_{G}(T)}\right)^{r}$ is the preimage of $\{1\} \times T w \subset \check{T} N_{G}(T) \times \check{T} N_{G}(T)$.

Let us again describe the action on $U$ :

$$
\left(a, f_{1}, f_{2}\right)(l, \hat{t} w)=\left(a l, s_{2}\left(f_{2}\right) s_{1}\left(f_{1}^{w}\right) \varphi\left(\left(f_{1}^{w}\right)^{-1}\right) s_{2}\left(a^{w}\right) \hat{t} s_{1}\left(a^{w}\right) \varphi\left(\left(a^{w}\right)^{-1}\right) w\right),
$$

where we write the action of Weyl group elements as exponents. We define a character $\lambda: \check{T} F \rightarrow U(1)$ by

$$
\lambda(\check{t} f):=s_{1}(\check{t} f) s_{2}(\check{t} f) \varphi(\check{t} f)^{-1} .
$$

By our choice of $s_{1}$ and $s_{2}$ we have $\lambda_{\mid F} \equiv 1$.

Then using the relation (2.14) in the form $\hat{t}^{s_{2}\left(a^{w}\right)}=B\left(a^{w}, t\right)^{-1} \hat{t}$ (where we must take the inverse $B\left(a^{w}, t\right)^{-1}$ since we consider the dual bundle $\hat{T}^{*} \rightarrow T$ ) we can write this action in the form

$$
\left(a, f_{1}, f_{2}\right)(l, \hat{t} w)=\left(a l, s_{2}\left(f_{2}\left(f_{1}^{w}\right)^{-1}\right) \hat{t} B\left(a^{w}, t\right)^{-1} \lambda\left(a^{w}\right)^{-1} w\right) .
$$

We trivialize $\hat{T} \cong T \times U(1)$ using a split $\kappa: T \rightarrow \hat{T}$. Let $\tilde{\kappa}: \hat{T} \rightarrow U(1)$ be the associated projection. We can now describe

$$
\left.U \cong\left[\operatorname{Lie}(T) \times \sqcup_{w \in W} T w \times U(1)\right] / \check{T} \times F \times F\right]
$$

with the action

$$
\left(a, f_{1}, f_{2}\right)(l, t w, z)=\left(a l, f_{2}\left(f_{1}^{w}\right)^{-1} t, \tilde{\kappa}\left(s_{2}\left(f_{2}\left(f_{1}^{w}\right)^{-1}\right)\right)^{-1} \lambda\left(a^{w}\right)^{-1} B\left(a^{w}, t\right)^{-1} z\right) .
$$

For $w \in W$ we consider the homomorphism $\kappa_{w}: F \times F \rightarrow F \times F$ given by $\left(f_{1}, f_{2}\right) \mapsto\left(f_{1}^{w^{-1}}, f_{1} f_{2}\right)$. Let $U_{w} \rightarrow P_{w}$ be the restriction of $U$ to the component labeled by $w \in W$. Then we have

$$
\begin{aligned}
\kappa_{w}^{*} U_{w} \cong[\operatorname{Lie}(T) \times(F \backslash T) \times U(1) / \check{T}] \otimes[U(1) / F] \\
\kappa_{w}^{*} P_{w} \cong[\operatorname{Lie}(T) \times F \backslash T / \check{T}] \times[* / F],
\end{aligned}
$$

where the action of $f \in F$ on $U(1)$ is trivial, and the action of $\check{T}$ on $\operatorname{Lie}(T) \times$ $(F \backslash T) \times U(1)$ is here given by

$$
a(l, F t, z)=\left(a l, F t, \omega\left(a^{w}\right)^{-1} B\left(a^{w}, F t\right)^{-1} z\right) .
$$

Moreover we can identify $P_{w} \cong[T \times(F \backslash T)] \times[* / F]$. The projection $v_{w}$ : $\kappa_{w}^{*} P_{w} \rightarrow[* / F \times F]$ is represented on the level of groups by $f \mapsto(f, 1)$. 
2.6.15. We have the associated line bundle

$$
\Psi_{w}:=(\operatorname{Lie}(T) \times F \backslash T \times \mathbb{C}) / \check{T} \rightarrow T \times(F \backslash T),
$$

where the action is given as in $(2.26)$. We must calculate $v_{!}\left[\Psi_{w}\right] \in K(*) \cong \mathbb{Z}$, where $v: T \times(F \backslash T) \rightarrow *$ is the projection. Let $X(\check{T})$ be the group of characters. The map

$$
b_{w}: F \backslash T \rightarrow X(\check{T})
$$

which is given by $b_{w}(F t)(\check{t})=B\left(\check{t}^{w}, F t\right)^{-1}$ is a bijection by Lemma 2.15 .

We see that we can identify $\Psi_{w} \rightarrow T \times F \backslash T$ with the Poincare bundle $\mathscr{P} \rightarrow T \times X(\check{T})$. It is well-known that $\tilde{v}_{!}(\mathscr{P})=1$, where $\tilde{v}: T \times X(\check{T}) \rightarrow *$ and $X(\check{T})$ is oriented as the dual torus to $T$. We therefore get $v_{!}\left(\Psi_{w}\right)= \pm 1$, where the sign depends on whether $b_{w}$ preserves the orientation or reverses it. We have $v_{!}\left(\Psi_{w}\right)= \pm \operatorname{sign}(w) v_{!}\left(\Psi_{1}\right)$, where the sign \pm only depends on the twist.

2.6.16. Let $z: * \rightarrow[* / F]$ be the projection. Then $z_{!}(1)=\sum_{\chi \in \hat{F}} \chi \in R(F)$. We conclude that $\left(v_{w}\right)_{!}\left(U_{w}\right)= \pm \operatorname{sign}(w) \sum_{\chi \in \hat{F}} 1 \otimes \chi \in R(F \times F)$. Note that

$$
\kappa_{w}^{*}\left(\sum_{\chi \in \hat{F}} \chi^{w^{-1}} \otimes \chi^{-1}\right)=\sum_{\chi \in \hat{F}} 1 \otimes \chi
$$

We conclude that

$$
q ! \circ p_{!}(U)= \pm \sum_{w \in W} \operatorname{sign}(w) \sum_{\chi \in \hat{F}} \chi^{w^{-1}} \otimes \chi^{-1} .
$$

Note that it suffices to sum over $\hat{F}^{r e g} \subset \hat{F}$.

2.6.17. Let us assume that

$$
D=\sum_{\left[\chi_{1}\right],\left[\chi_{2}\right] \in X_{1}^{r e g}(\hat{T}) / \hat{W}} a_{\left[\chi_{1}\right],\left[\chi_{2}\right]} E_{\chi_{1} \times \chi_{2}}
$$

with coefficients $a_{\left[\chi_{1}\right],\left[\chi_{2}\right]} \in \mathbb{Z}$ to be determined. Then by Theorem 2.17 we have by equation $(2.18)$

$$
\Theta(D)=\sum_{\left[\chi_{1}\right],\left[\chi_{2}\right] \in X_{1}^{r e g}(\hat{T}) / \hat{W}} a_{\left[\chi_{1}\right],\left[\chi_{2}\right]} \sum_{w_{1}, w_{2} \in W} \operatorname{sign}\left(w_{1}\right) \operatorname{sign}\left(w_{2}\right)\left(\tilde{s}^{*}\left(\chi_{1} \times \chi_{2}\right)\right)^{\left(w_{1}, w_{2}\right)} .
$$

We have (see 2.6.12 for notation)

$$
\left(\tilde{s}^{*}\left(\chi_{1} \times \chi_{2}\right)\right)^{\left(w_{1}, w_{2}\right)}=\left(s_{0}^{*} \chi_{1}\right)^{w_{1}} \otimes\left(\left(s_{0}^{\prime}\right)^{*} \chi_{2}^{-1}\right)^{w_{2}} .
$$

Now observe that $\left(s_{0}^{\prime}\right)^{*} \chi^{-1}=\left(s_{0}^{*} \chi^{-1}\right) \omega^{-1}=s_{0}^{*}(\omega \chi)^{-1}$ for $\chi \in X_{1}(\hat{T})$. 
2.6.18. We further rewrite

$$
\begin{aligned}
q_{!} \circ p_{!}(U) & = \pm \sum_{w_{1}, w_{2} \in W} \operatorname{sign}\left(w_{1}\right) \sum_{[\chi] \in \hat{F}^{r e g} / W}\left(\chi^{w_{2}}\right)^{w_{1}^{-1}} \otimes\left(\chi^{w_{2}}\right)^{-1} \\
& = \pm \sum_{w_{1}, w_{2} \in W} \operatorname{sign}\left(w_{1}\right) \operatorname{sign}\left(w_{2}\right) \sum_{[\chi] \in \hat{F}^{r e g} / W} \chi^{w_{1}} \otimes\left(\chi^{w_{2}}\right)^{-1} .
\end{aligned}
$$

We want to evaluate the equality $\Theta(D)=q_{!} p_{!}(U)$ which explicitly has the form

$$
\begin{aligned}
& \pm \sum_{w_{1}, w_{2} \in W} \operatorname{sign}\left(w_{1}\right) \operatorname{sign}\left(w_{2}\right) \sum_{[\chi] \in \hat{F}^{r e g} / W} \chi^{w_{1}} \otimes\left(\chi^{w_{2}}\right)^{-1} \\
& =\sum_{\left[\chi_{1}\right],\left[\chi_{2}\right] \in X_{1}^{r e g}(\hat{T}) / \hat{W}} a_{\left[\chi_{1}\right],\left[\chi_{2}\right]} \sum_{w_{1}, w_{2} \in W} \operatorname{sign}\left(w_{1}\right) \operatorname{sign}\left(w_{2}\right)\left(s_{0}^{*} \chi_{1}\right)^{w_{1}} \otimes\left(s_{0}^{*}\left(\omega \chi_{2}\right)^{-1}\right)^{w_{2}} .
\end{aligned}
$$

In view of the discussion in 2.4 .5 we see that

$$
\pm a_{\left[\chi_{1}\right],\left[\chi_{2}\right]}=\delta_{\left[\chi_{1}\right],\left[\omega \chi_{2}\right]} .
$$

This finishes the proof of Theorem 2.23.

\section{Moduli spaces and trivializations of twists}

\subsection{Stacks associated to polarized Hilbert spaces.}

3.1.1. Let $H=H_{+} \oplus H_{+}^{\perp}$ be a polarized Hilbert space, and let $P_{+}$denote the orthogonal projection onto $H_{+}$. In this situation we define the restricted unitary group

$$
U_{\text {res }}\left(H, H_{+}\right):=\left\{U \in U(H) \mid\left[U, P_{+}\right] \text {trace class }\right\} .
$$

We obtain the basic central extension of the connected component of the identity of $U_{\text {res }}\left(H, H_{+}\right)$in the following canonical way (see $\left.[\mathbf{P S 8 6}]\right)$. We first consider the subgroup

$$
E:=\left\{(U, Q) \in U_{\text {res }}\left(H, H_{+}\right) \times U\left(H_{+}\right) \mid P_{+} U P_{+}-Q \text { trace class }\right\} .
$$

This group sits in an extension

$$
0 \rightarrow T \rightarrow E \rightarrow U_{\text {res }}^{0}\left(H, H_{+}\right) \rightarrow 0,
$$

where $T=\left\{Q \in U\left(H_{+}\right) \mid 1-Q\right.$ trace class $\}$. Let det $: T \rightarrow U(1)$ be the Fredholm determinant. Then we define

$$
\hat{U}_{\text {res }}^{0}\left(H, H_{+}\right):=E \times_{T, \operatorname{det}} U(1) .
$$


Definition 3.1. This is the basic central extension:

$$
0 \rightarrow U(1) \rightarrow \hat{U}_{\text {res }}^{0}\left(H, H_{+}\right) \rightarrow U_{\text {res }}^{0}\left(H, H_{+}\right) \rightarrow 0 .
$$

3.1.2. Let $U^{H_{+}} \subset U_{\text {res }}^{0}\left(H, H_{+}\right)$be the subgroup of isometries fixing $H_{+}$. Then we have a natural split

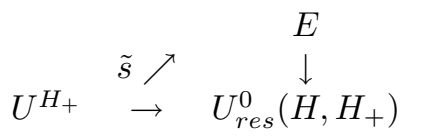

given by $\tilde{s}(u):=\left(u, P_{+} u P_{+}\right)$. It induces a split

$$
\begin{array}{ccc} 
& & \hat{U}_{\text {res }}^{0}\left(H, H_{+}\right) \\
U^{H_{+}} \stackrel{\downarrow}{\rightarrow} & U_{\text {res }}^{0}\left(\stackrel{H}{H}, H_{+}\right)
\end{array} .
$$

3.1.3. For completeness we explain how one can extend the central extension from $U_{\text {res }}^{0}\left(H, H_{+}\right)$to $U_{\text {res }}\left(H, H_{+}\right)$. Recall that $U_{\text {res }}\left(H, H_{+}\right)$comes as a (noncanonically split) extension (semi-direct product)

$$
0 \rightarrow U_{\text {res }}^{0}\left(H, H_{+}\right) \rightarrow U_{\text {res }}\left(H, H_{+}\right) \rightarrow \mathbb{Z} \rightarrow 0 .
$$

Choose a unitary $\sigma \in U_{\text {res }}\left(H, H_{+}\right)$with $\sigma\left(H_{+}\right) \subset H_{+}$and $\operatorname{codim}_{H_{+}} \sigma\left(H_{+}\right)=$ 1. We can then define a split $\mathbb{Z} \ni 1 \mapsto \sigma \in U_{\text {res }}\left(H, H_{+}\right)$. We extend $\sigma \in$ $\operatorname{Aut}\left(U_{\text {res }}^{0}\left(H, H_{+}\right)\right)$to an automorphism $\tilde{\sigma} \in \operatorname{Aut}\left(\hat{U}_{\text {res }}^{0}\left(H, H_{+}\right)\right)$by $\tilde{\sigma}[(U, Q), z]=$ $\left[\left(\sigma U \sigma^{-1}, Q_{\sigma}\right), z\right]$, where $[U, Q] \in E$ and

$$
Q_{\sigma}:=\left\{\begin{array}{cc}
\sigma Q \sigma^{-1} & \text { on } \sigma\left(H_{+}\right) \\
1 & \text { on } H_{+} \ominus \sigma\left(H_{+}\right)
\end{array} .\right.
$$

We then define

$$
\hat{U}_{\text {res }}\left(H, H_{+}\right):=\hat{U}_{\text {res }}^{0}\left(H, H_{+}\right) \rtimes \mathbb{Z} .
$$

This definition depends up to isomorphism on the choice of $\sigma$ and is therefore less canonical than the construction of the extension of the connected component.

3.1.4. The upshot of the preceeding discussion is that a polarized Hilbert space $H=H_{+} \oplus H_{+}^{\perp}$ gives rise to a twist

$$
\mathscr{T}:\left[* / \hat{U}_{\text {res }}\left(H, H_{+}\right)\right] \rightarrow\left[* / U_{\text {res }}\left(H, H_{+}\right)\right]
$$

whose restriction to the identity component of the restricted unitary group is canonical. 
3.1.5. We now consider the restricted Grassmannian

$$
G r_{r e s}\left(H, H_{+}\right):=\left\{P \text { orthogonal projection } \mid P-P_{+} \text {trace class }\right\} .
$$

It is a homogeneous space of $U_{\text {res }}\left(H, H_{+}\right)$. We have the pull-back

$$
\begin{array}{ccc}
{\left[G r_{\text {res }}\left(H, H_{+}\right) / \hat{U}_{\text {res }}\left(H, H_{+}\right)\right]} & \stackrel{p^{*} \mathscr{T}}{\rightarrow} & {\left[G r_{\text {res }}\left(H, H_{+}\right) / U_{\text {res }}\left(H, H_{+}\right)\right]} \\
p \downarrow & & \downarrow \\
{\left[* / \hat{U}_{\text {res }}\left(H, H_{+}\right)\right]} & \stackrel{\mathscr{T}}{\rightarrow} & {\left[* / U_{\text {res }}\left(H, H_{+}\right)\right]}
\end{array}
$$

Lemma 3.4. The twist $p^{*} \mathscr{T}$ is trivialized.

Proof. The Grassmannian $G r_{r e s}\left(H, H_{+}\right)$carries a determinant bundle $L \rightarrow G r_{r e s}\left(H, H_{+}\right)$. It was shown in [PS86], 7.7.3, that the central extension $\hat{U}_{\text {res }}\left(H, H_{+}\right)$acts canonically ${ }^{(1)}$ on $L$ lifting the action of $U_{\text {res }}\left(H, H_{+}\right)$on $G r_{\text {res }}\left(H, H_{+}\right)$. This line bundle gives the isomorphism $l: 0 \stackrel{\sim}{\rightarrow} p^{*} \mathscr{T}$.

3.1.6. Later we need the following fact. Recall that $U^{H_{+}} \subset U_{r e s}^{0}\left(H, H_{+}\right)$is the subgroup of transformations which fix $P_{+}$. Via the canonical split (3.3) its acts on the fibre of $L$ over $P_{+}$.

Lemma 3.5. The group $U^{H_{+}}$acts trivially on the fibre of $L$ over $P_{+}$.

\subsection{Moduli spaces.}

3.2.1. Let $C$ be a non-empty oriented one-dimensional closed Riemannian manifold. Observe that $C$ admits a natural action of $S^{1}$. If $V$ is a finitedimensional complex Hilbert space, then we consider the Hilbert space $H:=$ $L^{2}(C, V)$. The group $S^{1}$ acts on $V$ in a natural way. We obtain a polarization $H=H_{+} \oplus H_{+}^{\perp}$ be taking for $H_{+}$the subspace of non-negative Fourier modes.

3.2.2. Let now $G$ be a compact Lie group. Then we consider the trivial $G$ principal bundle $P(C)$ over $C$. Furthermore, we let $F(C)$ and $G(C)$ denote the space of flat connections and the gauge group of $P(C)$. The group $G(C) \cong$ $C^{\infty}(C, G)$ acts on $F(C)$. In this way we obtain the stack

$$
\mathscr{M}(C):=[F(C) / G(C)] .
$$

This generalizes the construction given in 2.1.1.

\footnotetext{
(1) Note that the definition of $\hat{U}_{\text {res }}\left(H, H_{+}\right)$and the definition of the action depend on the same choice of $\sigma$ (see 3.1.3)
} 
3.2.3. Let us now assume that $G$ acts unitarily on the Hilbert space $V$. We obtain an induced homomorphism $G(C) \rightarrow U(H)$. It is well-known (see [PS86], Sec. 6.3) that this homomorphism factors over the restricted unitary group $U_{\text {res }}\left(H, H_{+}\right)$. If $\pi_{1}(G)$ is finite, then $G(C)$ maps to $U_{r e s}^{0}\left(H, H_{+}\right)$.

3.2.4. We have an induced map of stacks $v: \mathscr{M}(C) \rightarrow\left[* / U_{\text {res }}\left(H, H_{+}\right)\right]$which can be used in order to define the twist (see 3.1.4 for the definition of $\mathscr{T}$ )

$$
v^{*} \mathscr{T}: \hat{\mathscr{M}}(C) \rightarrow \mathscr{M}(C) .
$$

The stack $\hat{\mathscr{M}}(C)$ is isomorphic to $[F(C) / \hat{G}(C)]$, where $\hat{G}(C) \rightarrow G(C)$ is the $U(1)$-central extension obtained as pull-back of $\hat{U}_{\text {res }}\left(H_{+}, H\right) \rightarrow U_{\text {res }}\left(H, H_{+}\right)$ via the homomorphism $G(C) \rightarrow U_{\text {res }}\left(H, H_{+}\right)$. If $\pi_{1}(G)$ is finite, then $v^{*} \mathscr{T}$ is canonical. In general at least the isomorphism class of $v^{*} \mathscr{T}$ is well-defined.

3.2.5. Recall the notion of admissibility 2.1.6.

Lemma 3.6. The twist $v^{*} \mathscr{T}$ is admissible.

Proof. It suffices to consider the case $C=S^{1}$. Note that $G \subset G\left(S^{1}\right)$ preserves $H_{+}$. We therefore have a factorization $G \rightarrow U^{H^{+}} \rightarrow U_{\text {res }}\left(H, H_{+}\right)$. Using 3.1 .2 we obtain a split $G \rightarrow \hat{G}$. In particular, then central extension $\widehat{N_{G}(T)} \rightarrow N_{G}(T)$ is trivial.

By an explicit calculation using the definitions in the case of $S U(n)$ and pulling back the result to $G$ we show that the extension $\widehat{\breve{T}} \rightarrow \check{T}$ is also trivial.

3.2.6. The extension $\check{T} T \subset G\left(S^{1}\right)$ determines a bilinear form $B: \check{T} \otimes T \rightarrow U(1)$ (see 2.4.2). In the present subsection we calculate this form. Note that $B$ is completely determined by its derivative $b: \check{T} \times \operatorname{Lie}(T) \rightarrow \operatorname{Lie}\left(S^{1}\right) \cong \mathbb{R}$ with respect to the second entry. We consider this as a homomorphism $b: \check{T} \rightarrow$ Lie $(T)^{*}$. Note that $\check{T}$ is a lattice in $\operatorname{Lie}(T)$. Thus $b$ has a unique extension to a linear map $b: \operatorname{Lie}(T) \rightarrow \operatorname{Lie}(T)^{*}$. This is a bilinear form on $\operatorname{Lie}(T)$.

An explicit calculation using the definitions yields the following formula.

Lemma 3.7. For $G=S U(n)$ and its standard representation on $\mathbb{C}^{n}$ the form $b$ is given by $b(X, Y)=\operatorname{Tr}(X Y)$.

From this we immediately obtain the general case if $\pi_{1}(G)$ is finite. In fact, in this case $\rho: G \rightarrow S U(V)$. Let $\rho: \operatorname{Lie}(G) \rightarrow \operatorname{Lie}(V)$ be the derived representation of Lie algebras. Then we have

$$
b(X, Y)=\operatorname{Tr}_{V}(\rho(X) \rho(Y)) .
$$


3.2.7. The form $(X, Y) \mapsto \operatorname{Tr}(X Y)$ on $\operatorname{Lie}(S U(n))$ is negative definite. It follows that its restriction to the Lie algebra of the maximal torus of $S U(n)$ is non-degenerated. We conclude:

Corollary 3.8. If $\rho: \operatorname{Lie}(G) \rightarrow \operatorname{Lie}(S U(n))$ is injective, then the form $b$ on $\mathrm{Lie}(T)$ is non-degenerated. In particular, the twist $v^{*} \mathscr{T}$ is regular (see 2.1.6).

3.2.8. Let $-C$ be $C$ equipped with the opposite orientation. Note that $\mathscr{M}(-C)=\mathscr{M}(C)$. Let $\bar{V}$ be the complex conjugated representation to $V$. In the following we indicate the dependence of the Hilbert spaces $H$ on $V$ by writing $H(V)$. Since $H_{+}$also depends on the orientation of $C$ we will write $H_{+}(V, C)$. As in 3.2.4 we have maps $v: \mathscr{M}(C) \rightarrow\left[* / U_{\text {res }}\left(H(V), H_{+}(V, C)\right)\right]$ and $\bar{v}: \mathscr{M}(C) \rightarrow\left[* / U_{\text {res }}\left(H(\bar{V}), H_{+}(\bar{V},-C)\right)\right]$ which induce twists $\tau$ an $\bar{\tau}$ of $\mathscr{M}(C)$.

Let us assume that $\pi_{1}(G)$ is finite.

Lemma 3.9. There exists a canonical isomorphism $\bar{\tau}=-\tau$.

Proof. The conjugate linear isomorphism $V \stackrel{\text { conj }}{\cong} \bar{V}$ induces a conjugated linear isomorphism $H(V) \stackrel{\text { conj }}{\cong} H(\bar{V})$ which identifies $H_{+}(V, C)$ with $H_{+}(\bar{V},-C)$. We obtain a corresponding diagram of groups

$$
\begin{gathered}
\hat{U}_{\text {res }}^{0}\left(H(V), H_{+}(V, C)\right)^{*} \cong \hat{U}_{\text {res }}^{0}\left(H(\bar{V}), H_{+}(\bar{V},-C)\right) \\
\downarrow \\
U_{\text {res }}^{0}\left(H(V), H_{+}(V, C)\right) \cong U_{\text {res }}^{0}\left(H(\bar{V}), H_{+}(\bar{V},-C)\right)
\end{gathered}
$$

Here for an $U(1)$-central extension $\hat{A} \rightarrow A$ we denote by $\hat{A}^{*} \rightarrow A$ the opposite extension. If $A$ acts on a space $X$, then the twist $\left[X / \hat{A}^{*}\right] \rightarrow[X / A]$ is the negative of $[X / \hat{A}] \rightarrow[X / A]$. The assertion now follows.

3.2.9. Let us assume a decomposition $C=C_{1} \cup C_{2}$. This induces decompositions $H=H_{1} \oplus H_{2}$ and $H_{+}=H_{1,+} \oplus H_{2,+}$. As in 3.2.4 we consider the maps $v_{i}: \mathscr{M}\left(C_{i}\right) \rightarrow\left[* / U_{\text {res }}\left(H_{i}, H_{+, i}\right)\right]$ and define the twists $\tau_{i}:=v_{i}^{*} \mathscr{T}_{i}$. Let $\tau:=v^{*} \mathscr{T}$.

Let us again assume that $\pi_{1}(G)$ is finite.

Lemma 3.10. We have a canonical isomorphism

$$
\mathrm{pr}_{1}^{*} \tau_{1}+\mathrm{pr}_{2}^{*} \tau_{2}=\tau
$$


Proof. We have a natural embedding

$$
U_{\text {res }}\left(H_{1}, H_{1,+}\right) \times U_{\text {res }}\left(H_{2}, H_{2,+}\right) \rightarrow U_{\text {res }}\left(H, H_{+}\right) .
$$

Let $U_{r e s}^{0}\left(H_{1}, H_{1,+} \widehat{\widehat{\times} U_{r e s}^{0}}\left(H_{2}, H_{2,+}\right) \rightarrow U_{r e s}^{0}\left(H_{1}, H_{1,+}\right) \times U_{r e s}^{0}\left(H_{2}, H_{2,+}\right)\right.$ be the induced central extension. It follows from the construction 3.1.1 that we have a canonical identification

$$
\left(\hat{U}_{\text {res }}^{0}\left(H_{1}, H_{+, 1}\right) \times \hat{U}_{\text {res }}^{0}\left(H_{2}, H_{+, 2}\right)\right) / U(1) \cong U_{r e s}^{0}\left(H_{1}, H_{1,+}\right) \times U_{r e s}^{0}\left(H_{2}, H_{2,+}\right) .
$$

Now in general, let $A$ and $B$ be groups acting on spaces $X$ and $Y$, respectively. Furthermore let $\hat{A} \rightarrow A$ and $\hat{B} \rightarrow B$ be $U(1)$-central extensions inducing twists $\alpha:[X / \hat{A}] \rightarrow[X / A]$ and $\beta:[Y / \hat{B}] \rightarrow[Y / B]$. Then the twist $\operatorname{pr}_{A}^{*} \alpha+\mathrm{pr}_{B}^{*} \beta$ is represented by $[X \times Y /((\hat{A} \times \hat{B}) / U(1))] \rightarrow[X \times Y / A \times B]$, where $\mathrm{pr}_{A}$, pr ${ }_{B}$ are the obvious projections.

This implies the result since we have a factorization of $v$ as

$$
\begin{aligned}
\mathscr{M}(C) \cong & \mathscr{M}\left(C_{1}\right) \times \mathscr{M}\left(C_{2}\right) \stackrel{v_{1} \times v_{2}}{\longrightarrow} \\
& {\left[* / U_{\text {res }}^{0}\left(H_{1}, H_{+, 1}\right)\right] \times\left[* / U_{\text {res }}^{0}\left(H_{2}, H_{+, 2}\right)\right] \rightarrow\left[* / U_{\text {res }}^{0}\left(H, H_{+}\right)\right] . }
\end{aligned}
$$

3.2.10. Let now $\Sigma$ be a two-dimensional oriented Riemannian manifold with non-empty boundary $\partial \Sigma$. Then we consider the trivial $G$-principal bundle $P(\Sigma)$ over $\Sigma$. Furthermore, we let $F(\Sigma)$ and $G(\Sigma)$ denote the space of flat connections and the gauge group of $P(\Sigma)$. The group $G(\Sigma)$ acts on $F(\Sigma)$. In this way we obtain the stack

$$
\mathscr{M}(\Sigma):=[F(\Sigma) / G(\Sigma)] .
$$

3.2.11. Evaluation at $\partial \Sigma$ defines a homomorphism $G(\Sigma) \rightarrow G(\partial \Sigma)$ and an equivariant map $F(\Sigma) \rightarrow F(\partial \Sigma)$. In this way we get a map of stacks

$$
q: \mathscr{M}(\Sigma) \rightarrow \mathscr{M}(\partial \Sigma) .
$$

3.2.12. We fix a unitary representation $V$ of $G$. Note that $\partial \Sigma$ is compact, oriented and Riemannian. Therefore we have a twist $v^{*} \mathscr{T}: \hat{\mathscr{M}}(\partial \Sigma) \rightarrow \mathscr{M}(\partial \Sigma)$.

Proposition 3.11. The pull-back of twists $q^{*} v^{*} \mathscr{T}$ is trivialized.

Proof. The Riemannian metric together with the orientation of $\Sigma$ gives a complex structure on $\Sigma$. A connection $A \in F(\Sigma)$ induces a holomorphic structure $\bar{\partial}_{A}$ on the associated bundle $V(\Sigma):=P(\Sigma) \times_{G} V$. We let $H(A) \subset$ $H=L^{2}(\partial \Sigma, V)$ denote the closure of the space of boundary values of continuous 
$\bar{\partial}_{A}$-holomorphic sections of $V(\Sigma)$. Let $P(A)$ be the projection onto $H(A)$. It turns out that $P(A) \in G r_{r e s}\left(H, H_{+}\right)$. We thus obtain a map

$$
P: F(\Sigma) \rightarrow G r_{r e s}\left(H, H_{+}\right)
$$

We now observe that this map is $G(\Sigma)$-equivariant, where $G(\Sigma)$ acts on the right-hand side via its homomorphism

$$
G(\Sigma) \rightarrow G(\partial \Sigma) \rightarrow U_{\text {res }}\left(H, H_{+}\right)
$$

Eventually we obtain the diagram of maps of stacks

$$
\begin{array}{cccc}
\mathscr{M}(\Sigma) & \stackrel{P}{\rightarrow} & {\left[G r_{\text {res }}\left(H, H_{+}\right) / U_{\text {res }}\left(H, H_{+}\right)\right]} \\
q \downarrow & & p \downarrow \\
\mathscr{M}(\partial \Sigma) & \stackrel{v}{\rightarrow} & {\left[* / U_{\text {res }}\left(H, H_{+}\right)\right]}
\end{array}
$$

The required trivialization is now given by

$$
P^{*} l: 0 \stackrel{\sim}{\rightarrow} P^{*} p^{*} \mathscr{T} \cong q^{*} v^{*} \mathscr{T}
$$

with $l$ obtained in Lemma 3.4 .

3.2.13. Let $C$ be a compact oriented one-dimensional Riemannian manifold. We consider two orientation and metric preserving embeddings $f_{0}, f_{1}:(-1,1) \times$ $C \rightarrow \Sigma$ with disjoint images. Then we can cut $\Sigma$ at the images $f_{i}(\{0\} \times C)$ and glue again interchanging the copies. In this way we obtain a compact oriented Riemannian two-manifold $\tilde{\Sigma}$ again with two embeddings $\tilde{f}_{0}, \tilde{f}_{1}:(-1,1) \times C$. Note that there is a canonical identification $\partial \Sigma \cong \partial \tilde{\Sigma}$.

3.2.14. We let $F(\Sigma, \sim) \subset F(\Sigma)$ be the space of flat connections $A$ on $\Sigma$ with the property that $f_{0}^{*} A=f_{1}^{*} A$. We define $F(\tilde{\Sigma}, \sim) \subset F(\tilde{\Sigma})$ in a similar manner. Then we have a canonical identification $F(\Sigma, \sim) \cong F(\tilde{\Sigma}, \sim)$.

We further define $G(\Sigma, \sim) \subset G(\Sigma)$ as the subgroup of gauge transformations $g$ satisfying $f_{0}^{*} g=f_{1}^{*} g$. We define $G(\tilde{\Sigma}, \sim) \subset G(\tilde{\Sigma})$ in a similar manner and observe that we have a canonical identification $G(\Sigma, \sim) \cong G(\tilde{\Sigma}, \sim)$.

3.2.15. We get a diagram of maps of stacks

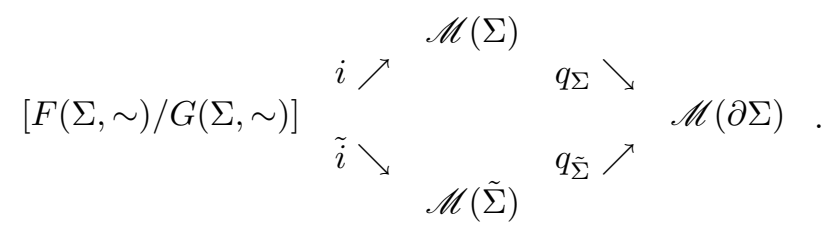


3.2.16. In Proposition 3.11 we have constructed trivializations

$$
\begin{array}{lll}
t(\Sigma) & : & q_{\Sigma}^{*} \mathscr{T} \stackrel{\sim}{\rightarrow} 0 \\
t(\tilde{\Sigma}) & : & q_{\tilde{\Sigma}}^{*} \mathscr{T} \stackrel{\sim}{\rightarrow} 0 .
\end{array}
$$

Note that

canonically.

$$
i^{*} q_{\Sigma}^{*} \mathscr{T} \cong \tilde{i}^{*} q_{\tilde{\Sigma}}^{*} \mathscr{T}
$$

Proposition 3.13. There exists an isomorphism of trivializations

$$
i^{*} t(\Sigma) \cong \tilde{i}^{*} t(\tilde{\Sigma})
$$

of $i^{*} q_{\Sigma}^{*} \mathscr{T} \cong \tilde{i}^{*} q_{\Sigma}^{*} \mathscr{T}$.

Proof. Recall that the trivializations $t\left(h^{\Sigma}\right)$ and $t\left(h^{\tilde{\Sigma}}\right)$ were induced by the equivariant bundles $P^{*} L$ and $\tilde{P}^{*} L$ (see 3.4 and 3.12 for the notation, and . . indicates objects associated to $\tilde{\Sigma})$. It suffices to show that $i^{*} P^{*} L$ and $\tilde{i}^{*} \tilde{P}^{*} L$ are isomorphic as $\hat{G}(\Sigma, \sim)$-equivariant bundles, where the central extension $\hat{G}(\Sigma, \sim) \rightarrow G(\Sigma, \sim)$ is defined as the restriction of $\hat{G}(\Sigma) \rightarrow G(\Sigma)$, and the latter is pulled back from $\hat{G}(\partial \Sigma) \rightarrow G(\partial \Sigma)$ (see 3.2.4). Therefore the following Lemma implies the proposition.

Lemma 3.14. The maps $i \circ P$ and $\tilde{i} \circ \tilde{P}$ are $G(\Sigma, \sim)$-equivariantly homotopic.

Proof. Let $A \in F(\Sigma, \sim)$ and $\bar{\partial}_{A}$ and $\widetilde{\bar{\partial}_{A}}$ be the corresponding holomorphic structures on $V(\Sigma)$ and $V(\tilde{\Sigma})$. It is a by now standard trick (see [Bun95]) to identify the spaces $C(\Sigma, V(\Sigma))$ and $C(\tilde{\Sigma}, V(\tilde{\Sigma}))$ in a $G(\Sigma, \sim)$-equivariant way so that $\delta:=\bar{\partial}_{A}-\widetilde{\bar{\partial}_{A}}$ is a zero-order (non-local) operator. For $t \in[0,1]$ we can form the projection $P_{t}(A)$ onto the boundary values of solutions of $\bar{\partial}_{A}-t \delta$. It provides the homotopy from $P(i(A))$ to $\tilde{P}(\tilde{i}(A))$.

3.2.17. In the following paragraphs we interpret the trivialization constructed in 3.11 and the surgery invariance 3.13 in a slightly different way. Let $\Sigma$ be a an oriented compact surface with Riemannian metric and nonempty boundary. We assume a decomposition of the boundary into an ingoing and an outgoing part:

$$
\partial \Sigma=\partial_{i} \Sigma \cup \partial_{a} \Sigma
$$

We will equip the ingoing boundary with the orientation which is opposite to the induced orientation. 
3.2.18. We assume that $\pi_{1}(G)$ is finite and fix an unitary representation $V$ of $G$. The construction 3.2 .4 gives rise to twists $\tau_{i}: \hat{\mathscr{M}}\left(\partial_{i} \Sigma\right) \rightarrow \mathscr{M}\left(\partial_{i} \Sigma\right)$ and $\tau_{a}: \hat{\mathscr{M}}\left(\partial_{a} \Sigma\right) \rightarrow \mathscr{M}\left(\partial_{a} \Sigma\right)$. We consider the correspondence

$$
\mathscr{M}\left(\partial_{i} \Sigma\right) \stackrel{q_{i}}{\leftarrow} \mathscr{M}(\Sigma) \stackrel{q_{a}}{\longrightarrow} \mathscr{M}\left(\partial_{a} \Sigma\right) .
$$

Lemma 3.16. We have a canonical isomorphism $r: q_{a}^{*} \tau_{a} \stackrel{\sim}{\rightarrow} q_{i}^{*} \tau_{i}$.

Proof. We can write $q=\left(q_{i}, q_{a}\right): \mathscr{M}(\Sigma) \rightarrow \mathscr{M}\left(\partial_{i} \Sigma\right) \times \mathscr{M}\left(\partial_{a} \Sigma\right) \cong \mathscr{M}(\partial \Sigma)$. Let $\mathrm{pr}_{i}: \mathscr{M}(\partial \Sigma) \rightarrow \mathscr{M}\left(\partial_{i} \Sigma\right)$ and $\mathrm{pr}_{a}: \mathscr{M}(\partial \Sigma) \rightarrow \mathscr{M}\left(\partial_{a} \Sigma\right)$ be the projections. By Lemma 3.10 and Lemma 3.9 we have a canonical isomorphism $\tau=\operatorname{pr}_{a}^{*} \tau_{a}-\mathrm{pr}_{i}^{*} \tau_{i}$, where $\tau$ is the twist of $\mathscr{M}(\partial \Sigma)$ given by 3.2.4. By Proposition 3.11 we have a canonical trivialization $q^{*} \tau \stackrel{\sim}{\rightarrow} 0$. If we add the identity $q_{i}^{*} \tau_{i} \cong q_{i}^{*} \tau_{i}$, then we obtain an isomorphism $r: q_{a}^{*} \tau_{a} \stackrel{\sim}{\rightarrow} q_{i}^{*} \tau_{i}$.

3.2.19. In this subsection we reinterpret the surgery invariance 3.13 . We keep the assumption that $\pi_{1}(G)$ is finite. We consider two compact oriented surfaces $\Sigma_{n}, n=0,1$ with non-empty boundary which are equipped with Riemannian metrics. We assume product structures near the boundaries. We assume an orientation reversing isometry $\psi: \partial_{i} \Sigma_{1} \stackrel{\sim}{\rightarrow} \partial_{a} \Sigma_{0}$. Then we can form the com-

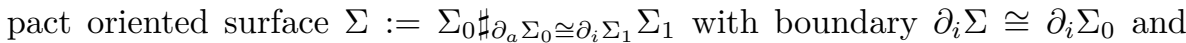
$\partial_{a} \Sigma \cong \partial_{a} \Sigma_{1}$. It comes equipped with an induced Riemannian metric.

We extend the notation introduced in 3.2 .18 by an index $\alpha \in\{0,1\}$ in order to indicate the surface to which the objects belong. We consider the following diagram

$$
\begin{array}{ccccc}
\mathscr{M}(\Sigma) & \stackrel{j}{\longrightarrow} & \mathscr{M}\left(\Sigma_{1}\right) & \stackrel{q_{1, a}}{\longrightarrow} & \mathscr{M}\left(\partial_{a} \Sigma_{1}\right) \\
i \downarrow & & \psi \circ q_{1, i} \downarrow & & \\
\mathscr{M}\left(\Sigma_{0}\right) & \stackrel{q_{0, a}}{\longrightarrow} & \mathscr{M}\left(\partial_{a} \Sigma_{0}\right) & & \\
q_{0, i} \downarrow & & & \\
\mathscr{M}\left(\partial_{i} \Sigma_{0}\right) & & &
\end{array}
$$

where $i$ and $j$ are the canonical restriction maps. The following is just a rewriting of 3.13

Corollary 3.18. We have a commutative diagram

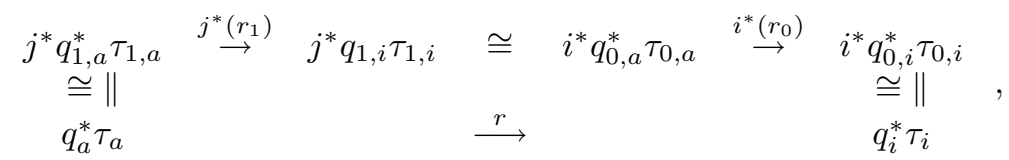

where all maps denoted by $\cong$ are canonical identifications. 


\subsection{The product.}

3.3.1. In the present subsection we shall assume that $\pi_{1}(G)$ is finite. This implies that the twists constructed in 3.2.4 and their trivializations 3.11 are canonical.

Moreover we assume that the twist $\sigma(G)$ introduced in 2.2.12 is trivial (see 2.2.14). This will imply that various maps used below are $K$-orientable.

We fix an orientation of the vector space Lie $(G)$.

3.3.2. We consider the correspondence of stacks

$$
[G \times G / G \times G] \stackrel{p}{\leftarrow}[G \times G / G] \stackrel{q}{\rightarrow}[G / G],
$$

where $p$ is induced by the identity on the level of spaces, and by the diagonal embedding of groups, and $q$ is given by the multiplication on the level of spaces, and by the identity on the level of groups. We consider a twist of $[G / G]$ of the form $\tau=\mathrm{hol}_{*} v^{*} \mathscr{T}$. By considering an equivalent correspondence of moduli spaces associated to a pair of pants surface and 3.16 we will obtain an isomorphism of twists

$$
r: q^{*} \tau \stackrel{\sim}{\rightarrow} p^{*}\left(\operatorname{pr}_{1}^{*} \tau+\mathrm{pr}_{2}^{*} \tau\right)
$$

We will furthermore construct a $K$-orientation of the proper and representable map $q$ such that

$$
m:{ }^{\tau} K([G / G]) \otimes{ }^{\tau} K([G / G]) \rightarrow{ }^{\tau} K([G / G])
$$

defined by

$$
m(x, y):=q ! r^{*} p^{*}\left(\mathrm{pr}_{1}^{*} x \cup \mathrm{pr}_{2}^{*} y\right)
$$

is an associative unital product. In fact, its complexification will coincide with the product induced by the identification ${ }^{\tau} K([G / G])_{\mathbb{C}} \cong R(G)_{\mathbb{C}} / I$ given in 2.19 .

3.3.3. Note that $[G / G] \rightarrow[* / G]$ is $-\sigma(G)$ - $K$-orientable (see 2.2.12). By our assumption on $G$ we have $\sigma(G)=0$. Then $[G / G] \rightarrow[* / G]$ is $K$-orientable. Therefore $[G \times G / G \times G] \rightarrow[* / G \times G] K$-orientable. By restriction to the diagonal subgroup we see that $[G \times G / G] \rightarrow[* / G]$ is $K$-orientable. It follows that $q$ is $K$-orientable.

3.3.4. Let now $\Sigma$ be an oriented pair of pants surface with ingoing boundary components $\partial_{i, \alpha} \Sigma, \alpha=1,2$, and outgoing boundary component $\partial_{a} \Sigma$. We assume that $\Sigma$ comes with a Riemannian metric which has a product structure near the boundary such the boundary circles are isometric to standard circles. The correspondence

$$
\mathscr{M}\left(\partial_{i} \Sigma\right) \stackrel{p}{\leftarrow} \mathscr{M}(\Sigma) \stackrel{q}{\rightarrow} \mathscr{M}\left(\partial_{a} \Sigma\right)
$$


is equivalent to (3.19). Lemma 3.16 together with 3.10 now gives the desired isomorphism of twists

$$
r: p^{*} \tau_{i} \stackrel{\sim}{\rightarrow} q^{*} \tau_{a},
$$

where we use the notation of 3.2.18. Note that this isomorphism may depend on the choice of the identification of the correspondence (3.19) with the correspondence (3.21).

3.3.5. We fix base points $b_{\alpha} \in \partial_{i, \alpha} \Sigma, \alpha \in\{1,2\}$, and $b \in \partial_{a} \Sigma$. Using the orientation of $\partial_{i} \Sigma$ opposite to the induced one we define the holonomy map

$$
\mathrm{hol}_{i}: \mathscr{M}\left(\partial_{i} \Sigma\right) \rightarrow[G / G] \times[G / G] .
$$

Let $\mathrm{hol}_{a}: \mathscr{M}\left(\partial_{a} \Sigma\right) \rightarrow[G / G]$ be the holonomy map associated to the outgoing boundary component. The projection $[G / G] \rightarrow[* / G]$ induces a $R(G)$-module structure on ${ }^{\tau} K([G / G])$. Via the two projections

$$
[G \times G / G \times G] \rightarrow[G / G] \rightarrow[* / G]
$$

we have two $R(G)$-module structures on $\operatorname{pr}_{1}^{*} \tau+\operatorname{pr}_{2}^{*} \tau K([G \times G / G \times G])$, which we write as left- and right actions. Using the identifications via the holonomy maps we obtain corresponding actions on ${ }^{\tau_{a}} K\left(\mathscr{M}\left(\partial_{a} \Sigma\right)\right)$ and ${ }^{\tau_{i}} K\left(\mathscr{M}\left(\partial_{i} \Sigma\right)\right)$.

Lemma 3.22. The multiplication map $m$ is $R(G)$-bilinear.

Proof. This is an immediate consequence of the commutativity of the diagram

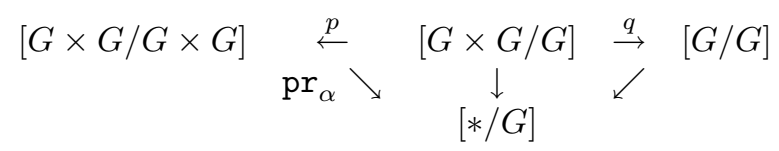

and the projection formula (see 2.2.9).

3.3.6. In the proof of Lemma 2.19 we have identified ${ }^{\tau} K([G / G])_{\mathbb{C}} \cong R(G)_{\mathbb{C}} / I$ with the space of sections $\Gamma(S, V)$, where $V \rightarrow F^{r e g} / W$ is a one-dimensional vector bundle associated to the character sign : $W \rightarrow\{1,-1\}$, and $S \subset$ $F^{r e g} / W$. Therefore $\operatorname{pr}_{1}^{*} \tau+\operatorname{pr}_{2}^{*} \tau K([G \times G / G \times G])_{\mathbb{C}} \cong \Gamma\left(S \times S, \operatorname{pr}_{1}^{*} V \otimes \operatorname{pr}_{2}^{*} V\right)$ with the left and right $\mathbb{C}[S]$-module structures induced by the two projections $\mathrm{pr}_{\alpha}: F^{r e g} \times F^{r e g} \rightarrow F^{r e g}$. The multiplication thus induces a linear map

$$
m_{\mathbb{C}}: \Gamma\left(S \times S, \operatorname{pr}_{1}^{*} V \otimes \operatorname{pr}_{2}^{*} V\right) \rightarrow \Gamma(S, V) .
$$

Such a map is given by structure constants $C_{r, s}^{t} \in \operatorname{Hom}\left(V_{r} \otimes V_{s}, V_{t}\right), r, s, t \in S$.

Since $m$ is $\mathbb{C}[S]$-bilinear we immediately conclude that $C_{r, s}^{t}=0$ if not $t=$ $s=r$. 
We define the section $c \in \Gamma\left(S, V^{*}\right)$ by $c_{s}:=C_{s, s}^{s}, s \in S$, where $V^{*}$ is the dual bundle.

Note that all twisted $K$-groups are free $\mathbb{Z}$-modules and therefore embed in their complexifications. In order to determine the product (3.20) it therefore suffices to calculate its complexification, i.e, the section $c$. The following properties follow from the vanishing of the off-diagonal structure constants.

Corollary 3.23. The product (3.20) is associative and commutative.

These properties in particular are independent on the choice of the $K$ orientation of $q$ and the choice of the isomorphism of twists $r$ (see 3.3.4 for the notation).

3.3.7. In this subsection let us write $\Sigma_{1}$ for the pair of pants surface considered above, $q_{1, a}$ for $q$, and $q_{1, i}$ for $p$. We furthermore consider an oriented surface $\Sigma_{0}$ which is the union of a disk and a cylinder such that it has an ingoing boundary component (belonging to the cylinder) and two outgoing boundary components. We equip $\Sigma_{0}$ with Riemannian metric which has a product structure such that the boundary components are isometric to the standard circle. We fix an orientation reversing isometry $\psi: \partial_{i} \Sigma_{1} \stackrel{\sim}{\rightarrow} \partial_{a} \Sigma_{0}$ such that $\partial_{i, 2} \Sigma_{1}$ is mapped to the boundary of the disk. This is exactly the situation considered in 3.2.19. Let $\Sigma:=\Sigma_{0} \sharp \partial_{a} \Sigma_{0} \cong \partial_{i} \Sigma_{1} \Sigma_{1}$ be the surface obtained by glueing. Then $\Sigma$ is a cylinder.

The map $q_{0, a}: \mathscr{M}\left(\Sigma_{0}\right) \rightarrow \mathscr{M}\left(\partial_{a} \Sigma_{0}\right)$ is equivalent to

$$
[* / G] \times[G / G] \rightarrow[G / G] \times[G / G] \cong[G \times G / G \times G]
$$

and therefore proper, representable and $K$-orientable. In fact, the choice of an orientation of $\operatorname{Lie}(G)$ induces a $K$-orientation of $q_{0, a}$.

Observe that the square in (3.17) is cartesian. Therefore the $K$-orientation of $q_{0, a}$ induces a $K$-orientation of $j$, and together with the choice of a $K$ orientation of $q_{1, a}$ a $K$-orientation of

$$
q_{a}:=q_{1, a} \circ j: \mathscr{M}(\Sigma) \rightarrow \mathscr{M}\left(\partial_{a} \Sigma\right) .
$$

Using Corollary 3.18 we obtain the identity of maps ${ }^{\tau} K([G / G]) \rightarrow{ }^{\tau} K([G / G])$.

$$
\begin{aligned}
m \circ\left(q_{0, a}\right) ! \circ r_{0}^{*} \circ q_{0, i}^{*} & =\left(q_{1, a}\right) ! \circ r_{1}^{*} \circ q_{1, i}^{*} \circ \psi^{*} \circ\left(q_{0, a}\right) ! \circ r_{0}^{*} \circ q_{0, i}^{*} \\
& =\left(q_{1, a}\right) ! \circ r_{1}^{*} \circ j_{!} \circ i^{*} \circ r_{0}^{*} \circ q_{0, i}^{*} \\
& =\left(q_{1, a}\right) ! \circ j_{!} \circ j^{*}\left(r_{1}\right)^{*} \circ i^{*}\left(r_{0}\right)^{*} \circ i^{*} \circ q_{0, i}^{*} \\
& =\left(q_{a}\right) ! \circ r^{*} \circ q_{i}^{*},
\end{aligned}
$$

where $r$ is associated to the cylinder. Note that the correspondence (3.15) associated to a cylinder is equivalent to

$$
[G / G] \stackrel{q_{i}}{\longleftarrow}[G / G] \stackrel{q_{a}}{\longrightarrow}[G / G],
$$


where all maps are the identity. In particular, there is a distinguished $K$ orientation of $q_{a}$, and a distinguished isomorphism of twists $q_{a}^{*} \tau \stackrel{\sim}{\rightarrow} q_{i}^{*} \tau$.

The set of $K$-orientations of $q_{a}$ (up to sign) is then identified with

$$
H^{2}([G / G], \mathbb{Z}) \cong H^{2}\left(G \times_{G} E G, \mathbb{Z}\right) \subset H^{2}(G, \mathbb{Z}) \cong \operatorname{Ext}\left(\pi_{1}(G), \mathbb{Z}\right)
$$

(Leray-Serre spectral sequence). Since $q_{1, a} \circ j$ is an isomorphism, we see that $j^{*}: H^{2}([G \times G / G], \mathbb{Z}) \rightarrow H^{2}([G / G], \mathbb{Z})$ is surjective. Therefore we can choose the $K$-orientation of $q_{1, a}$ such that the induced $K$-orientation of $q_{a}=q_{1, a} \circ j$ is the distinguished one up to a sign.

Using again the surjectivity of $j^{*}$ we can adjust the isomorphism of twists $r_{1}$ such that the induced isomorphism twists $r: q_{a}^{*} \tau \stackrel{\sim}{\rightarrow} q_{i}^{*} \tau$ is the distinguished one.

We now fix the sign of the $K$-orientation of $q_{1, a}$ such that the induced orientation of $q_{a}$ is the distinguished one. In this case $\left(q_{a}\right) ! \circ r^{*} \circ q_{i}^{*}=i d$. This fixes also the class of $K$-orientations used to define the product $m$.

3.3.8. Let $\Sigma^{D} \subset \Sigma_{0}$ be the component of the disk. We indicate the related maps with the same superscript. We have an element $E:=\left(q_{a}^{D}\right) ! \circ\left(r^{D}\right)^{*}(1)$. The correspondence (3.15) associated to the disk is equivalent to

$$
* \leftarrow[* / G] \stackrel{q_{a}^{D}}{\rightarrow}[G / G] .
$$

Therefore the element $E$ is the same as the one constructed 2.5.1. The construction of $E$ in 2.5.1 depends on the choice of a section $G \rightarrow \hat{G}$. Under the present assumptions on $G$ there is only one such section, since $G$ has no non-trivial $U(1)$-valued characters. In the present subsection we will see why we called $E$ the unit.

In fact the calculation 3.24 and commutativity of the product 3.23 now gives

$$
m(E, x)=m(x, E)=x, \quad \forall x \in{ }^{\tau} K([G / G]) .
$$

We immediately conclude that the section $c$ is invertible and determined by

$$
E \cong c^{-1}
$$

where we consider $E$ and $c^{-1}$ as sections of $V$. The description of the product in terms of the basis $\left(E_{[\chi]}\right)_{[\chi] \in X_{1}(\hat{T}) / \hat{W}}$ is more complicated.

3.3.9. We can state the final theorem about the product. We adopt the choices of $K$-orientations fixed above.

Theorem 3.25. The product $m$ induces on ${ }^{\tau} K([G / G])$ a commutative and associative ring structure with identity $E$. Its complexification is isomorphic to the quotient $R(G)_{\mathbb{C}} / I$. 


\section{References}

[AS04] M. Aтіyah \& G. Segal - Twisted K-theory, 2004, arXiv:math.KT/0407054.

[Bun95] U. Bunke - On the gluing problem for the $\eta$-invariant, J. Differential Geom. 41 (1995), no. 2, p. 397-448.

[FHT02] D. Freed, M. J. Hopkins \& C. Teleman - Twisted equivariant $K$-theory with complex coefficients, 2002, arXiv:math.AT/0206257.

[FHT03] _ Twisted $K$-theory and Loop group representations I, 2003, arXiv:math.AT/0312155.

[Hei05] J. Heinloth - Notes on differentiable Stacks, 2005, this volume.

[Pos03] H. Posthuma - Quantization of Hamiltonian loop group actions, 2003, PhD-thesis, Amsterdam.

[PS86] A. Pressley \& G. Segal - Loop groups, Oxford Mathematical Monographs, The Clarendon Press Oxford University Press, New York, 1986, Oxford Science Publications.

[TXL03] J. Tu, P. Xu \& C. LAurent - Twisted $K$-theory of differentiable stacks, 2003, arXiv:math.KT/0306318. 
Mathematisches Institut, Seminars, (Y. Tschinkel, ed.), p. 81-92

Universität Göttingen, 2004-05

\section{UNIVERSALITY OF $L$-FUNCTIONS}

\section{J. Steuding}

Departamento de Matemáticas, Universidad Autónoma de Madrid, C.

Universitaria de Cantoblanco, 28049 Madrid, Spain

E-mail : jorn.steuding@uam.es

Abstract. We survey recent results on the value-distribution of $L$-functions with emphasis on aspects of universality.

\section{Voronin's theorem}

In 1975 Voronin [Vor75b] discovered a remarkable analytical property of the Riemann zeta-function $\zeta(s)$. Roughly speaking, he proved that any nonvanishing analytic function can be approximated uniformly by certain purely imaginary shifts of the zeta-function in the right half of the critical strip. After significant improvements due to Reich [Rei77] and Bagchi [Bag81] the strongest version of Voronin's theorem has the form (see [Lau96]):

Theorem 1. Suppose that $\mathscr{K}$ is a compact subset of the strip $\mathscr{D}:=\{s \in$ $\left.\mathbb{C}: \frac{1}{2}<\operatorname{Re} s<1\right\}$ with connected complement, and let $g(s)$ be a non-vanishing continuous function on $\mathscr{K}$ which is analytic in the interior of $\mathscr{K}$. Then, for any $\varepsilon>0$,

$$
\liminf _{T \rightarrow \infty} \frac{1}{T} \text { meas }\left\{\tau \in[0, T]: \max _{s \in \mathscr{K}}|\zeta(s+i \tau)-g(s)|<\varepsilon\right\}>0 .
$$

October 26, 2004. 
We may interpret the absolute value of an analytic function as an analytic landscape over the complex plane. Then the universality theorem states that any (finite) analytic landscape can be found (up to an arbitrarily small error) in the analytic landscape of $\zeta(s)$. This remarkable property has several interesting consequences on the value-distribution of the zeta-function. For instance, for any $n \in \mathbb{N}$ and any fixed $s$ with $\operatorname{Re} s \in\left(\frac{1}{2}, 1\right)$ the set

$$
\left\{\left(\zeta(s+i \tau), \zeta^{\prime}(s+i \tau), \ldots, \zeta^{(n-1)}(s+i \tau)\right): \tau \in \mathbb{R}\right\}
$$

is dense in $\mathbb{C}^{n}$. Moreover, it follows that the zeta-function does not satisfy any algebraic differential equation.

In the first half of the twentieth century, Harald Bohr applied probabilistic methods in order to study the value distribution of $\zeta(s)$. Voronin's proof of his universality theorem relies heavily on Bohr's ideas. Here we sketch a variant of the proof in the language of weakly convergent probability measures due to Bagchi [Bag81].

Denote by $\gamma=\{s \in \mathbb{C}:|s|=1\}$ the unit circle in the complex plane and put

$$
\Omega=\prod_{p} \gamma_{p}
$$

where $\gamma_{p}=\gamma$ for each prime number $p$. With product topology and pointwise multiplication this infinite dimensional torus $\Omega$ is a compact topological abelian group, and hence the normalized Haar measure $m$ on the metric space $(\Omega, \mathscr{B}(\Omega))$ exists; here $\mathscr{B}(\Omega)$ denotes the class of Borel sets of $\Omega$. This induces a probability space $(\Omega, \mathscr{B}(\Omega), \mathrm{m})$. Let $\omega(p)$ denote the projection of $\omega \in \Omega$ on the coordinate space $\gamma_{p}$. Since the Haar measure $\mathrm{m}$ on $\Omega$ is the product of the Haar measures $\mathrm{m}_{p}$ on the coordinate spaces, $\{\omega(p): p$ prime $\}$ is a sequence of independent complex-valued random variables defined on $(\Omega, \mathscr{B}(\Omega), \mathrm{m})$. Now denote by $\mathscr{H}(\mathscr{D})$ the set of analytic functions defined on the strip $\mathscr{D}$, equipped with the topology of uniform convergence on compacta. For $\operatorname{Re} s>\frac{1}{2}$ and $\omega \in \Omega$, let

$$
\zeta(s, \omega)=\prod_{p}\left(1-\frac{\omega(p)}{p^{s}}\right)^{-1} .
$$

This defines an $\mathscr{H}(\mathscr{D})$-valued random element on the probability space $(\Omega, \mathscr{B}(\Omega), \mathrm{m})$. It can be shown that for almost all $\omega \in \Omega$ the infinite product defining $\zeta(s, \omega)$ converges uniformly on compact subsets of $\mathscr{D}$.

The first step in the proof of Theorem 1 is to establish the weak convergence of the probability measure $\mathbf{P}_{T}$, defined by

$$
\mathbf{P}_{T}(A)=\frac{1}{T} \operatorname{meas}\{\tau \in[0, T]: \zeta(s+i \tau) \in A\} \quad \text { for } \quad A \in \mathscr{B}(\mathscr{H}(\mathscr{D})),
$$


to the distribution $\mathbf{P}$ of the random element $\zeta(s, \omega)$, given by

$$
\mathbf{P}(A)=\mathrm{m}\{\omega \in \Omega: \zeta(s+i \tau) \in A\} \quad \text { for } \quad A \in \mathscr{B}(\mathscr{H}(\mathscr{D})),
$$

as $T \rightarrow \infty$. For short,

$$
\mathbf{P}_{T} \quad \Rightarrow \quad \mathbf{P} \quad(T \rightarrow \infty)
$$

The proof of this limit theorem relies on fundamental results from probabilty theory, e.g. Prokhorov's theorems, ergodic theory for random processes, and the simple but important fact that the logarithms of different prime numbers are linearly independent.

The support of the random element

$$
\log \zeta(s, \omega)=\sum_{p} \log \left(1-\frac{\omega(p)}{p^{s}}\right)^{-1}
$$

is the closure of the set of all convergent series

$$
\sum_{p} \log \left(1-\frac{a(p)}{p^{s}}\right)^{-1} \quad \text { with } \quad a(p) \in \gamma .
$$

The second step is to prove that the set of all these convergent series is dense in $\mathscr{H}\left(\mathscr{D}_{M}\right)$, where $\mathscr{D}_{M}:=\left\{s \in: \frac{1}{2}<\operatorname{Re} s<1,|\operatorname{Im} s|<M\right\}$ and $M$ is an arbitrary positive constant. This involves the theory of entire functions of exponential type, a rearrangement theorem in Hilbert spaces, and the prime number theorem. The map $f \mapsto \exp f$ sends $\mathscr{H}\left(\mathscr{D}_{M}\right)$ to

$$
\left\{g \in \mathscr{H}\left(\mathscr{D}_{M}\right): g(s) \neq 0 \quad \text { for } \quad \frac{1}{2}<\operatorname{Re} s<1\right\} .
$$

Now, roughly speaking, the limit theorem (1) ties both ends together. Since $\mathscr{K}$ is a compact subset of $\mathscr{D}$, there exists some $M$ for which $\mathscr{K} \subset \mathscr{D}_{M}$. It follows that any $g$ which is contained in the support of the random element $\zeta(s, \omega)$ and has a non-vanishing analytic continuation to $\mathscr{D}_{M}$ can be approximated uniformly by some shift $\zeta(s+i \tau)$ for $s \in \mathscr{K}$ : if $\Phi$ denotes the set of $\varphi \in \mathscr{H}$ such that

$$
\max _{s \in \mathscr{K}}|\varphi(s)-g(s)|<\varepsilon
$$

then

$$
\liminf _{T \rightarrow \infty} \frac{1}{T} \text { meas }\left\{\tau \in[0, T]: \max _{s \in \mathscr{K}}|\zeta(s+i \tau)-g(s)|<\varepsilon\right\} \geqslant \mathbf{P}(\Phi)>0 .
$$

The case of functions $g$ with zeros in $\mathscr{D}_{M} \backslash \mathscr{K}$ follows from an application of Mergelyan's approximation theorem). This yields the statement of Theorem 1. 
All known proofs of universality results for Dirichlet series, like the one for the zeta-function above or those covered by Theorem 2 below, depend on some arithmetical conditions. But is universality really an arithmetic phenomenon or not? Reviewing the proof one might understand universality as a kind of ergodicity on function spaces. It seems reasonable that the universality of Dirichlet series is a common phenomenon in analysis, that it is related to Julia rays in value-distribution theory and to ergodical dynamical systems as well.

\section{Zeros and the Riemann hypothesis}

Since any non-vanishing analytic function possesses an analytic logarithm, it follows that $\log \zeta(s)$ is strongly universal, i.e., $\log \zeta(s)$ can uniformly approximate functions having zeros; in fact, we have almost given a proof of this fact in the previous section. It is natural to ask whether $\zeta(s)$ is also strongly universal. The answer is negative. We give a heuristic argument which can be made waterproof with a bit more effort by the techniques of Section 5 .

Assume that $g(s)$ is an analytic function on $|s| \leqslant r$, where $0<r<\frac{1}{4}$, which has a zero $\xi$ with $|\xi|<r$ but which is non-vanishing on the boundary. Then, whenever the inequality

$$
\max _{|s| \leqslant r}\left|\zeta\left(s+\frac{3}{4}+i \tau\right)-g(s)\right|<\varepsilon<\min _{|s| \leqslant r}|g(s)| ;
$$

holds, $\zeta\left(s+\frac{3}{4}+i \tau\right)$ has to have a zero inside $|s| \leqslant r$. This can be seen as follows. By the maximum principle the maximum on the left hand side of the inequality above is taken on the boundary. The second inequality holds for sufficiently small $\varepsilon$ (since the zeros of an analytic function form a discrete set or the function vanishes identically). Consequently, an application of Rouché's theorem yields the existence of a zero of $\zeta\left(s+\frac{3}{4}+i \tau\right)$ inside $|s| \leqslant r$. If now for any $\varepsilon>0$

$$
\liminf _{T \rightarrow \infty} \frac{1}{T} \text { meas }\left\{\tau \in[0, T]: \max _{|s| \leqslant r}\left|\zeta\left(s+\frac{3}{4}+i \tau\right)-g(s)\right|<\varepsilon\right\}>0,
$$

then we expect $\gg T$ many complex zeros of $\zeta(s)$ in the strip $\frac{3}{4}-r<\operatorname{Re} s<\frac{3}{4}+r$ up to height $T$. This contradicts classical density estimates: for any $\sigma>\frac{1}{2}$, the number of zeros $\rho=\beta+i \gamma$ satisfying $\beta>\sigma, 0<\gamma \leqslant T$ is known to be $o(T)$ as $T \rightarrow \infty$. Thus, uniform approximation of a function $g(s)$ with a zero by the zeta-function is impossible.

Bohr [Boh22] discovered an interesting relation between the Riemann hypothesis and almost periodicity. He showed that if $\chi$ is non-principal character, then the Riemann hypothesis for the associated Dirichlet $L$-function $L(s, \chi)$ 
(i.e., the non-vanishing of $L(s, \chi)$ for $\left.\operatorname{Re} s>\frac{1}{2}\right)$ is equivalent to the almost periodicity of $L(s, \chi)$ in the half-plane $\operatorname{Re} s>\frac{1}{2}$. Because of the restriction on non-principal characters $\chi$ this result does not cover the case of $\zeta(s)$. More than half a century later Bagchi $[\mathbf{B a g} 81$ ] proved that the same criterion holds also for $\zeta(s)$, namely that Riemann's hypothesis is true if and only if for any compact subset $\mathscr{K}$ of the strip $\frac{1}{2}<\operatorname{Re} s<1$ with connected complement and for any $\varepsilon>0$

$$
\liminf _{T \rightarrow \infty} \frac{1}{T} \text { meas }\left\{\tau \in[0, T]: \max _{s \in \mathscr{K}}|\zeta(s+i \tau)-\zeta(s)|<\varepsilon\right\}>0 .
$$

The crucial implication of Bagchi's proof relies essentially on Voronin's universality theorem, which, of course, was unknown to Bohr.

\section{The Selberg class}

Meanwhile, it is known that there exists a rich zoo of universal Dirichlet series; for a list we refer to [Lau96], [Mat04], [Ste04]. It was conjectured by Linnik and Ibragimov that all functions given by Dirichlet series and analytically continuable to the left of the half plane of absolute convergence, which satisfy some natural growth conditions, are universal. In this section we are interested in the universality of $L$-functions.

In 1989 Selberg [Sel92] defined a general class $\mathscr{S}$ of Dirichlet series having an Euler product, analytic continuation and a functional equation of Riemanntype, and formulated some fundamental conjectures concerning them. His aim was to study the value-distribution of linear combinations of $L$-functions. In the meantime this so-called Selberg class became an important object of research. All known examples of functions in the Selberg class are automorphic (or at least conjecturally automorphic) $L$-functions, and for all of them it turns out that the related Euler factors are the inverse of a polynomial in $p^{-s}$. This special shape of the Euler product is related to Langlands' reciprocity conjecture. In the sequel we will consider a subclass of polynomial Euler products.

The class $\tilde{\mathscr{S}}$ consists of Dirichlet series

$$
\mathscr{L}(s):=\sum_{n=1}^{\infty} \frac{a(n)}{n^{s}}
$$

satisfying the following axioms: 
- Polynomial Euler product: for $1 \leqslant j \leqslant m$ and each prime $p$ there exist complex numbers $\alpha_{j}(p)$ with $\left|\alpha_{j}(p)\right| \leqslant 1$ such that

$$
\mathscr{L}(s)=\prod_{p} \prod_{j=1}^{m}\left(1-\frac{\alpha_{j}(p)}{p^{s}}\right)^{-1} ;
$$

- Mean-square: there exists a positive constant $\kappa$ such that

$$
\lim _{x \rightarrow \infty} \frac{1}{\pi(x)} \sum_{p \leqslant x}|a(p)|^{2}=\kappa ;
$$

- Analytic continuation: there exists a non-negative integer $k$ such that $(s-1)^{k} \mathscr{L}(s)$ is an entire function of finite order;

- Functional equation: there are positive real numbers $Q, \lambda_{j}$, and there are complex numbers $\mu_{j}, \omega$ with $\operatorname{Re} \mu_{j} \geqslant 0$ and $|\omega|=1$, such that

$$
\Lambda_{\mathscr{L}}(s)=\omega \overline{\Lambda_{\mathscr{L}}(1-\bar{s})},
$$

where

$$
\Lambda_{\mathscr{L}}(s):=\mathscr{L}(s) Q^{s} \prod_{j=1}^{f} \Gamma\left(\lambda_{j} s+\mu_{j}\right) .
$$

It should be noted that the axiom on the mean-square is intimately related to Selberg's conjectures (see [Sel92]). We expect that $\tilde{\mathscr{S}}$ contains all non-constant functions from the Selberg class: $\tilde{\mathscr{S}}=\mathscr{S} \backslash\{1\}$.

The degree of any non-constant function $\mathscr{L} \in \mathscr{S}$ (and so in $\tilde{\mathscr{S}}$ ) is defined by

$$
\mathrm{d} \mathscr{L}=2 \sum_{j=1}^{f} \lambda_{j} .
$$

This quantity is well-defined. If $N_{\mathscr{L}}(T)$ counts the number of zeros of $\mathscr{L} \in \mathscr{S}$ in the rectangle $0 \leqslant \operatorname{Re} s \leqslant 1,|\operatorname{Im} s| \leqslant T$ (counting multiplicities) one can show by standard contour integration

$$
N_{\mathscr{L}}(T) \sim \frac{\mathrm{d} \mathscr{L}}{\pi} T \log T
$$

in analogy to the classical Riemann-von Mangoldt formula for $\zeta(s)$. It is conjectured that the degree is always a positive integer (provided that $\mathscr{L}$ is not constant one).

The functions of degree one in $\tilde{\mathscr{S}}$ are the Riemann zeta-function and shifts of Dirichlet $L$-functions $L(s+i \theta, \chi)$ attached to primitive characters $\chi$ with $\theta \in \mathbb{R}$. Examples of degree two are normalized $L$-functions associated with holomorphic newforms; normalized $L$-functions attached to non-holomorphic 
newforms are expected to lie in $\tilde{\mathscr{S}}$. The Rankin-Selberg $L$-function of any two holomorphic newforms is an element of the Selberg class of degree 4. Further examples are Dedekind zeta-functions to number fields $\mathbb{K}$; their degree is equal to the degree of the field extension $\mathbb{K} / \mathbb{Q}$.

In $[\mathbf{S t e 0 4}]$ the following generalization of Voronin's universality theorem was proved.

Theorem 2. Let $\mathscr{L} \in \tilde{\mathscr{S}}$ and $\mathscr{K}$ be a compact subset of the strip

$$
\mathscr{D}_{\mathscr{L}}:=\left\{s \in \mathbb{C}: \max \left\{\frac{1}{2}, 1-\frac{1}{\mathrm{~d}_{\mathscr{L}}}\right\}<\operatorname{Re} s<1\right\}
$$

with connected complement, and let $g(s)$ be a non-vanishing continuous function on $\mathscr{K}$ which is analytic in the interior of $\mathscr{K}$. Then, for any $\varepsilon>0$,

$$
\liminf _{T \rightarrow \infty} \frac{1}{T} \text { meas }\left\{\tau \in[0, T]: \max _{s \in \mathscr{K}}|\mathscr{L}(s+i \tau)-g(s)|<\varepsilon\right\}>0 .
$$

This theorem may be regarded as a verification of the Linnik-Ibragimov conjecture for Dirichlet series in the Selberg class.

\section{The strip of universality}

Besides the arithmetic axioms on the polynomial Euler product and on the mean-square, a further important ingredient in the proof of Theorem 2 is the second moment bound

$$
\limsup _{T \rightarrow \infty} \frac{1}{T} \int_{1}^{T}|\mathscr{L}(\sigma+i t)|^{2} \mathrm{~d} t<\infty .
$$

In [Ste04] an asymptotic formula for $\mathscr{L} \in \tilde{\mathscr{S}}$ in the range $\mathscr{D} \mathscr{L}$ was proved. If $\mathrm{d}_{\mathscr{L}}>2$, the strip $\mathscr{D} \mathscr{L}$ does not cover the right half of the critical strip; any extension of this strip to the left would imply universality in this extended strip. However, the critical line is a natural boundary for universality of $\mathscr{L} \in \tilde{\mathscr{S}}$ (at least in the sense of Theorem 2).

In particular cases of functions $\mathscr{L} \in \tilde{\mathscr{S}}$ with degree $\mathrm{d} \mathscr{L}>2$ the existence of the mean-square covering the strip $\mathscr{D} \mathscr{L}$ is known. For instance, let $L(s, \chi)$ be an arbitrary Dirichlet $L$-function to a primitive character $\chi$. Then $\zeta(s)^{2} L(s, \chi)$ is an element of $\tilde{\mathscr{S}}$ of degree 3, so Theorem 2 gives universality for $\frac{2}{3}<\operatorname{Re} s<1$. Using Montgomery's estimates for the fourth moment of Dirichlet $L$-functions and Ivić's eigth-moment estimate for $\zeta(s)$, the Cauchy-Schwarz inequality yields

$$
\int_{1}^{T}\left|\zeta(\sigma+i t)^{2} L(\sigma+i t, \chi)\right|^{2} \mathrm{~d} t \ll T^{\frac{11-8 \sigma}{12}+\frac{1}{2}+\varepsilon} \ll T
$$


for any $\sigma>\frac{5}{8}$. Thus $\zeta(s)^{2} L(s, \chi)$ is universal in the strip $\frac{5}{8}<\operatorname{Re} s<1$. If the generalized Lindelöf hypothesis is true for $\mathscr{L} \in \tilde{\mathscr{S}}$, i.e.,

$$
\mathscr{L}\left(\frac{1}{2}+i t\right) \ll t^{\varepsilon}
$$

for any $\varepsilon>0$ as $t \rightarrow \infty$, then the strip of universality can be extended to the full open right half of the critical strip.

\section{Effectivity}

The known proofs of universality theorems are ineffective, giving neither an estimate for the first approximating shift $\tau$ nor bounds for the positive lower density. There are some remarkable attempts due to Garunkštis [Gar03], Good [Goo81], and Laurinčikas [Lau00], however, their results are either restricted to rather small classes of functions or conditional subject to certain unproved hypotheses. Following [Ste03] we now consider the problem of effective upper bounds for the upper density of universality.

Denote by $B_{r}$ the closed disc of radius $r>0$ with center in the origin. We define for a meromorphic function $L(s)$, an analytic function $g: B_{r} \rightarrow \mathbb{C}$ with fixed $r \in\left(0, \frac{1}{4}\right)$, and positive $\varepsilon$ the densities

$$
\underline{\mathrm{d}}(\varepsilon, g, L)=\liminf _{T \rightarrow \infty} \frac{1}{T} \operatorname{meas}\left\{\tau \in[0, T]: \max _{|s| \leqslant r}\left|L\left(s+\frac{3}{4}+i \tau\right)-g(s)\right|<\varepsilon\right\},
$$

and

$$
\overline{\mathrm{d}}(\varepsilon, g, L)=\limsup _{T \rightarrow \infty} \frac{1}{T} \operatorname{meas}\left\{\tau \in[0, T]: \max _{|s| \leqslant r}\left|L\left(s+\frac{3}{4}+i \tau\right)-g(s)\right|<\varepsilon\right\} .
$$

We consider analytic isomorphisms $g: B_{r} \rightarrow B_{1}$, i.e., the inverse $g^{-1}$ exists and is analytic. Obviously, such a function $g$ has exactly one simple zero $\xi$ in the interior of $B_{r}$. By the Schwarz lemma any such $g$ has a representation

$$
g(s)=r \exp (i \varphi) \frac{\xi-s}{r^{2}-\bar{\xi} s} \quad \text { with } \quad \varphi \in \mathbb{R} \quad \text { and } \quad|\xi|<r .
$$

Denote by $\mathscr{A}_{r}$ the class of analytic isomorphisms from $B_{r}$ (with fixed $0<r<\frac{1}{4}$ ) to the unit disc. Further, let $N_{L}\left(\sigma_{1}, \sigma_{2}, T\right)$ count the number of zeros of $L(s)$ in $\frac{1}{2}<\sigma_{1}<\operatorname{Re} s<\sigma_{2}<1,0 \leqslant t<T$ (counting multiplicities).

Theorem 3. Suppose that $g \in \mathscr{A}_{r}$. Assume that $L(s)$ is analytic in $\operatorname{Re} s \geqslant$ $\frac{3}{4}-r$ except for at most $o(T)$ many singularities inside $\operatorname{Re} s \geqslant \frac{3}{4}-r, 0 \leqslant$ 
$\operatorname{Im} s \leqslant T$, as $T \rightarrow \infty$, and that $\underline{\mathrm{d}}(\varepsilon, g, L)>0$ for all $\varepsilon>0$. Then, for any $\varepsilon \in\left(0, \frac{1}{2 r}\left(\frac{1}{4}+\operatorname{Re}|\xi|\right)\right)$,

$$
\overline{\mathrm{d}}(\varepsilon, g, L) \leqslant \frac{8 r^{3} \varepsilon}{r^{2}-|\xi|^{2}} \limsup _{T \rightarrow \infty} \frac{1}{T} N_{L}\left(\frac{3}{4}+\operatorname{Re} \xi-2 r \varepsilon, \frac{3}{4}+\operatorname{Re} \xi+2 r \varepsilon, T\right) .
$$

We sketch the proof (which is a bit in the spirit of Section 2). The zero $\xi$ of $g$ is related to some zeros of $L(s)$ in $\frac{1}{2}<\operatorname{Res} s<1$. Since $g$ maps the boundary of $B_{r}$ onto the unit circle, Rouché's theorem implies the existence of one simple zero $\lambda$ of $L(z)$ in

whenever

$$
K_{\tau}:=\left\{z=s+\frac{3}{4}+i \tau: s \in B_{r}\right\}
$$

$$
\max _{s \in B_{r}}\left|L\left(s+\frac{3}{4}+i \tau\right)-g(s)\right|<\varepsilon<1=\min _{s \in B_{r}}|g(s)| .
$$

We may say that the zero $\lambda$ of $L(s)$ is generated by the zero $\xi$ of $g(s)$. Universality is a phenomenon that happens in intervalls. Suppose that a zero $\lambda$ of $L(s)$, generated by $\xi$, lies in two different sets $K_{\tau_{1}}$ and $K_{\tau_{2}}$. Then one can show that

$$
\left|\tau_{1}-\tau_{2}\right|<\frac{8 r^{4} \varepsilon}{r^{2}-|\xi|^{2}}
$$

Now denote by $\mathscr{I}_{j}(T)$ the disjoint intervalls in $[0, T]$ such that (1) is valid exactly for $\tau \in \cup_{j} \mathscr{I}_{j}(T)=: \mathscr{I}(T)$. By the latter estimate, in every intervall $\mathscr{I}_{j}(T)$, there lie at least

$$
1+\left[\frac{r^{2}-|\xi|^{2}}{8 r^{3} \varepsilon} \operatorname{meas} \mathscr{I}_{j}(T)\right] \geqslant \frac{r^{2}-|\xi|^{2}}{8 r^{3} \varepsilon} \text { meas } \mathscr{I}_{j}(T)
$$

zeros $\lambda$ of $L(s)$ in the strip $\frac{1}{2}<\operatorname{Re} s<1$. Therefore, the number $\mathscr{N}(T)$ of such zeros $\lambda$ satisfies the estimate

$$
\frac{8 r^{3} \varepsilon}{r^{2}-|\xi|^{2}} \mathscr{N}(T) \geqslant \operatorname{meas} \mathscr{I}(T) .
$$

The value distribution of $L(z)$ in $K_{\tau}$ is ruled by that of $g(s)$ in $B_{r}$. This gives a restriction on the real parts of the zeros $\lambda$. One can show that $\left|\operatorname{Re} \lambda-\frac{3}{4}-\operatorname{Re} \xi\right|<2 r \varepsilon$. This yields

$$
\mathscr{N}(T) \leqslant N_{L}\left(\frac{3}{4}+\operatorname{Re} \xi-2 r \varepsilon, \frac{3}{4}+\operatorname{Re} \xi+2 r \varepsilon, T\right) .
$$

Since $\underline{\mathrm{d}}(\varepsilon, g, L)>0$, this leads via (2) to the estimate of the theorem. Note that the set of singularities of $L(s)$ in $\sigma \geqslant \frac{3}{4}-r$ has zero density but $\underline{\mathrm{d}}(\varepsilon, g, L)>0$. So the singularities do not affect the above observations. 
Theorem 3 relates the density of universality to the value-distribution of $L$. In the case of the Riemann zeta-function we can be more explicit. In view of classical density theorems the set of singularities of $\log \zeta(s)$ has density zero. Hence, we may apply Theorem to $L(s)=\log \zeta(s)$. By Bohr and Jessen [BJ32], Hilfssatz 6, the limit

$$
\lim _{T \rightarrow \infty} \frac{1}{T} N_{\log \zeta}\left(\frac{3}{4}+\operatorname{Re} \xi-2 r \varepsilon, \frac{3}{4}+\operatorname{Re} \xi+2 r \varepsilon, T\right)
$$

exists, and tends to zero as $\varepsilon \rightarrow 0$. Hence, under the above assumptions

$$
\overline{\mathrm{d}}(\varepsilon, \exp g, \zeta(s))=o(\varepsilon) .
$$

Thus, the decay of $\overline{\mathrm{d}}(\varepsilon, \exp g, \zeta)$ with $\varepsilon \rightarrow 0$ is more than linear in $\varepsilon$.

\section{Joint universality}

We conclude with another interesting problem concerning universality of $L$-functions.

Voronin [Vor75a] also obtained joint universality for Dirichlet $L$-functions, that is simultaneous uniform approximation by a family of $L$-functions associated with non-equivalent characters; the non-equivalence of the characters assures a certain independence of the related $L$-functions, and this independence is necessary for joint universality. Recently, Laurinčikas \& Matsumoto [LM04] proved a joint universality theorem for $L$-functions associated with newforms twisted by characters. It is natural to ask for joint universality in the Selberg class. However, all known jointly universal families are given by (multiplicative or additive) twists of a single universal Dirichlet series by characters. In some sense, Selberg's Conjecture B (see [Sel92]) states that primitive functions form an orthonormal system in the Selberg class. As proved by Bombieri \& Hejhal [BH95], this implies the statistical independence of primitive functions. There is some hope that this can be used as substitute for the independence induced by non-equivalent characters in order to prove joint universality for distinct primitive $L$-functions from the Selberg class.

For $1 \leqslant j \leqslant m$, assume that the $L$-functions

$$
\mathscr{L}_{j}(s)=\sum_{n=1}^{\infty} \frac{a_{\mathscr{L}_{j}}(n)}{n^{s}}
$$

from $\tilde{\mathscr{S}}$ satisfy the orthogonality condition

$$
\sum_{p \leqslant x} \frac{a_{\mathscr{L}_{j}}(p) \overline{a_{\mathscr{L}_{k}}(p)}}{p}=\delta_{j k} \kappa_{j} \log \log x+O(1),
$$


where $\kappa_{j}$ is a positive constant depending on $\mathscr{L}_{j}$, and $\delta_{j k}=1$ if $j=k$ and $\delta_{j k}=0$ otherwise. This condition is known to hold for several families of $L$ functions in $\tilde{\mathscr{S}}$, for example for Dirichlet $L$-functions associated with pairwise non-equivalent characters (in which case it is nothing else than the orthogonality relation for characters); it is expected to hold for any two distinct primitive $L$-functions from the Selberg class (Selberg's Conjecture B). Moreover, (3) may be regarded as an extension of the axiom on the mean square in the definition of $\tilde{\mathscr{S}}$.

Conjecture. Suppose that $\mathscr{L}_{1}, \ldots, \mathscr{L}_{m}$ are elements of $\tilde{\mathscr{S}}$ satisfying condition (3). For $1 \leqslant j \leqslant m$ let $g_{j}(s)$ be a continuous function on $\mathscr{K}_{j}$ which is nonvanishing in the interior, where $\mathscr{K}_{j}$ is a compact subset of the strip

$$
\mathscr{D}:=\left\{s: \max \left\{\frac{1}{2}, 1-\frac{1}{\mathrm{~d}}\right\}<\operatorname{Res}<1\right\}
$$

with connected complement, and $\mathrm{d}$ is the maximum of the degrees of the $\mathscr{L}_{j}$ (a quantity determined by the functional equation for $\left.\mathscr{L}_{j}\right)$. Then, for any $\varepsilon>0$,

$$
\liminf _{T \rightarrow \infty} \frac{1}{T} \operatorname{meas}\left\{\tau \in[0, T]: \max _{1 \leqslant j \leqslant m} \max _{s \in \mathscr{K}_{j}}\left|\mathscr{L}_{j}(s+i \tau)-g_{j}(s)\right|<\varepsilon\right\}>0 .
$$

Acknowledgements. The author is very grateful to Prof. Yuri Tschinkel for the invitation to Göttingen and the possibility of having a look into Riemann's original manuscripts on the zeta-function.

\section{References}

[Bag81] B. BAGCHI - The statistical behaviour and universality properties of the Riemann zeta-function and other allied Dirichlet series, 1981, Ph.D.Thesis, Calcutta, Indian Statistical Institute.

[BH95] E. Bombieri \& D. A. Hejhal - On the distribution of zeros of linear combinations of Euler products, Duke Math. J. 80 (1995), no. 3, p. 821862.

[BJ32] H. Bohr \& B. Jessen - Über die Werteverteilung der Riemannschen Zetafunktion, zweite Mitteilung, Acta Math. 58 (1932), p. 1-55.

[Boh22] H. BoHR - Über eine quasi-periodische Eigenschaft Dirichletscher Reihen mit einer Anwendung auf die Dirichletschen l-Funktionen, Math. Annalen 85 (1922), p. 115-122.

[Gar03] R. GARUnKŠTis - The effective universality theorem for the Riemann zeta function, in Proceedings of the Session in Analytic Number Theory and Diophantine Equations (Bonn), Bonner Math. Schriften, vol. 360, Univ. Bonn, 2003, p. 21. 
[Goo81] A. Good - On the distribution of the values of Riemann's zeta function, Acta Arith. 38 (1980/81), no. 4, p. 347-388.

[Lau96] A. LAURINČIKAS - Limit theorems for the Riemann zeta-function, Mathematics and its Applications, vol. 352, Kluwer Academic Publishers Group, Dordrecht, 1996.

[Lau00] A. LAURINCHIKAS - Effectivization of the universality theorem for the Lerch zeta function, Liet. Mat. Rink. 40 (2000), no. 2, p. 172-178.

[LM04] A. LAURINČIKAS \& K. Matsumoto - The joint universality of twisted automorphic L-functions, J. Math. Soc. Japan 56 (2004), no. 3, p. 923-939.

[Mat04] K. Mатsumoto - Probabilistic value-distribution theory of zeta-functions, Sugaku Expositions 17 (2004), no. 1, p. 51-71.

[Rei77] A. REICH - Universelle Werteverteilung von Eulerprodukten, Nachr. Akad. Wiss. Göttingen Math.-Phys. Kl. II (1977), no. 1, p. 1-17.

[Sel92] A. SELBERG - Old and new conjectures and results about a class of Dirichlet series, in Proceedings of the Amalfi Conference on Analytic Number Theory (Maiori, 1989) (Salerno), Univ. Salerno, 1992, p. 367-385.

[Ste03] J. Steuding - Upper bounds for the density of universality, Acta Arith. 107 (2003), no. 2, p. 195-202.

[Ste04] J. Steuding - Value-distribution of $L$-functions, 2004, habilitation thesis, Frankfurt.

[Vor75a] S. M. Voronin - The functional independence of Dirichlet $L$-functions, Acta Arith. 27 (1975), p. 493-503, Collection of articles in memory of Juriı Vladimirovič Linnik.

[Vor75b] _ A theorem on the "universality" of the Riemann zeta-function, $I z v$. Akad. Nauk SSSR Ser. Mat. 39 (1975), no. 3, p. 475-486, 703. 
Mathematisches Institut, Seminars, (Y. Tschinkel, ed.), p. 93-98

Universität Göttingen, 2004-05

\title{
MASSEYPRODUKTE IN DER GALOISKOHOMOLOGIE VON ZAHLKÖRPERN
}

\section{Vogel}

NWF I - Mathematik, Universität Regensburg, 93040 Regensburg, Germany

E-mail : denis.vogel@mathematik.uni-regensburg.de

\begin{abstract}
We study the connection between Massey products and relations in pro$p$-groups and give an arithmetical example, thereby obtaining a cohomological interpretation of the Rédei symbol.
\end{abstract}

\section{Masseyprodukte und Relationen in pro- $p$-Gruppen}

In diesem Abschnitt verallgemeinern wir den bekannten Zusammenhang zwischen dem Cupprodukt in der Kohomologie von pro- $p$-Gruppen und Darstellungen von pro- $p$-Gruppen durch Erzeugende und Relationen.

Sei $p$ eine Primzahl und $G$ eine endlich erzeugte pro- $p$-Gruppe. Wir werden im folgenden Gebrauch von den Kohomologiegruppen $H^{i}(G, \mathbb{Z} / p \mathbb{Z})$ machen und diese der Einfachheit halber mit $H^{i}(G)$ bezeichnen. Wir setzen $n=$ $\operatorname{dim}_{\mathbb{Z} / p \mathbb{Z}} H^{1}(G)$. Es ist wohlbekannt, daß dies der Erzeugendenrang von $G$ ist ([NSW00], Prop. 3.9.1). Sei

$$
1 \longrightarrow R \longrightarrow F \longrightarrow G \longrightarrow 1
$$

eine minimale Darstellung von $G$, wobei $F$ eine freie pro- $p$-Gruppe auf Erzeugern $x_{1}, \ldots, x_{n}$ sei. Aus der Hochschild-Serre-Spektralsequenz erhalten wir die exakte Sequenz

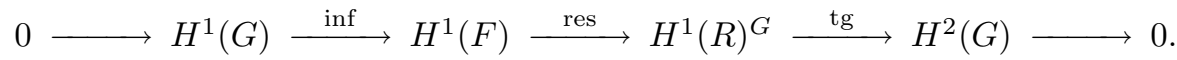

\footnotetext{
October 28, 2004.
} 
Aufgrund der Minimalität der obigen Darstellung ergibt sich, daß die Inflationsabbildung

$$
\inf : H^{1}(G) \rightarrow H^{1}(F)
$$

ein Isomorphismus ist, wir werden beide Gruppen im folgenden miteinander identifizieren. Insbesondere ist auch die Transgressionabbildung

$$
\operatorname{tg}: H^{1}(R)^{G} \rightarrow H^{2}(G)
$$

ein Isomorphismus. Für jedes Element $\rho \in R$ erhalten wir die sogenannte Spurabbildung

$$
\operatorname{tr}_{\rho}: H^{2}(G) \rightarrow \mathbb{Z} / p \mathbb{Z}, \varphi \mapsto\left(\operatorname{tg}^{-1} \varphi\right)(\rho) .
$$

Es sei $I$ der Kern der Augmentationsabbildung $\mathbb{F}_{p} \llbracket F \rrbracket \rightarrow \mathbb{F}_{p}$, wobei $\mathbb{F}_{p} \llbracket F \rrbracket$ die vollständige Gruppenalgebra von $F$ über $\mathbb{F}_{p}$ bezeichne. Durch

$$
F_{(m)}=\left\{f \in F \mid f-1 \in I^{m}\right\}
$$

ist eine Filtrierung auf $F$ gegeben, die sogenannte Zassenhaus-Filtrierung.

Es ist wohlbekannt, daß die Cupprodukt-Paarung

$$
H^{1}(G) \times H^{1}(G) \stackrel{\cup}{\rightarrow} H^{2}(G)
$$

Informationen über $R$ liefert: Bezeichnet $\chi_{1}, \ldots, \chi_{n}$ die zu $x_{1}, \ldots, x_{n}$ duale Basis von $H^{1}(F)=\operatorname{Hom}(F, \mathbb{Z} / p \mathbb{Z})$, dann gilt für jedes $\rho \in R$ die Gleichung ([NSW00], Prop. 3.9.13)

$$
\rho \equiv \prod_{k=1}^{n} x_{k}^{p b_{k k}} \prod_{1 \leqslant k<l \leqslant n}\left(x_{k}, x_{l}\right)^{b_{k l}} \bmod F_{(3)}
$$

(hierbei ist $\left(x_{k}, x_{l}\right)$ der Kommutator $\left.x_{k}^{-1} x_{l}^{-1} x_{k} x_{l}\right)$, mit

$$
\operatorname{tr}_{\rho}\left(\chi_{k} \cup \chi_{l}\right)=-b_{k l} \text {. }
$$

Wir werden im folgenden studieren, was passiert, wenn das Cupprodukt trivial ist. In diesem Fall gibt es Dreifach-Masseyprodukte, welche wie folgt definiert sind. Es seien $u_{1}, u_{2}, u_{3} \in H^{1}(G)$ mit $u_{1} \cup u_{2}=0, u_{2} \cup u_{3}=0$. Dann existieren 1-Koketten $u_{12}, u_{23}$, so daß auf dem Niveau von 2-Koketten

$$
u_{1} \cup u_{2}=\partial u_{12}, \quad u_{2} \cup u_{3}=\partial u_{23},
$$

gilt, und wir setzen

$$
\left\langle u_{1}, u_{2}, u_{3}\right\rangle=\left[u_{1} \cup u_{23}+u_{12} \cup u_{3}\right] \in H^{2}(G),
$$

wobei [·] die Kohomologieklasse des entsprechenden Kozykels bedeutet. Hierbei ist $\left\langle u_{1}, u_{2}, u_{3}\right\rangle$ unabhängig von den getroffenen Auswahlen. Dies verallgemeinernd kann man Masseyprodukte $\left\langle u_{1}, \ldots, u_{m}\right\rangle$ der Länge $m$ für $u_{1}, \ldots, u_{m} \in$ $H^{1}(G)$ definieren $([\mathbf{M o r 0 4}],[\mathbf{V o g}])$, im allgemeinen liegt $\left\langle u_{1}, \ldots, u_{m}\right\rangle$ jedoch 
in einem Quotienten von $H^{2}(G)$. Wir werden ein Kriterium angeben, wann $\left\langle u_{1}, \ldots, u_{m}\right\rangle$ in $H^{2}(G)$ liegt, und in solch einem Fall $\operatorname{tr}_{\rho}\left\langle u_{1}, \ldots, u_{m}\right\rangle$ berechnen. Dazu benötigen wir die Notationen des Foxschen Differentialkalküls. Bezeichnet $\mathbb{Z}_{p} \llbracket F \rrbracket$ die vollständige Gruppenalgebra von $F$ über $\mathbb{Z}_{p}$ und $\psi: \mathbb{Z}_{p} \llbracket F \rrbracket \rightarrow \mathbb{Z}_{p}$ die Augmentationsabbildung, dann existieren für jedes $i=1, \ldots, n$ eindeutig bestimmte Abbildungen

$$
\frac{\partial}{\partial x_{i}}: \mathbb{Z}_{p} \llbracket F \rrbracket \rightarrow \mathbb{Z}_{p} \llbracket F \rrbracket,
$$

die sogenannten freien Ableitungen, so daß für jedes $\alpha \in \mathbb{Z}_{p} \llbracket F \rrbracket$ die Gleichung

$$
\alpha=\psi(\alpha) 1_{\mathbb{Z}_{p} \llbracket F \rrbracket}+\sum_{i=1}^{n} \frac{\partial \alpha}{\partial x_{i}}\left(x_{i}-1\right)
$$

erfüllt ist ([Iha86]). Für $1 \leqslant i_{1}, \ldots, i_{m} \leqslant n$ definieren wir

$$
\varepsilon_{\left(i_{1}, \ldots, i_{m}\right)}: F \rightarrow \mathbb{Z}_{p}, f \mapsto \psi\left(\frac{\partial^{m} f}{\partial x_{i_{1}} \cdots \partial x_{i_{m}}}\right) \quad \bmod p
$$

Es gilt der folgende Satz ([Mor04],[Vog] $]$.

Satz 1.1. Es gilt $R \subseteq F_{(m)}$ genau dann, wenn alle Masseyprodukte bis zur Länge $m-1$ trivial sind. In diesem Fall liegen alle Masseyprodukte der Länge $m$ in $H^{2}(G)$, und für $u_{1}, \ldots, u_{m} \in H^{1}(G), \rho \in R$ gilt

$$
\operatorname{tr}_{\rho}\left\langle u_{1}, \ldots, u_{m}\right\rangle=(-1)^{m-1} \sum_{1 \leqslant i_{1}, \ldots, i_{m} \leqslant n} u_{1}\left(x_{i_{1}}\right) \cdot \ldots \cdot u_{m}\left(x_{i_{m}}\right) \varepsilon_{\left(i_{1}, \ldots, i_{m}\right)}(\rho),
$$

insbesondere also

$$
\operatorname{tr}_{\rho}\left\langle\chi_{i_{1}}, \ldots, \chi_{i_{m}}\right\rangle=(-1)^{m-1} \varepsilon_{\left(i_{1}, \ldots, i_{m}\right)}(\rho)
$$

Für $\rho \in F_{(m)}$ hängen die $\varepsilon_{\left(i_{1}, \ldots, i_{m}\right)}(\rho)$ eng mit dem Bild von $\rho$ in $F_{(m)} / F_{(m+1)}$ zusammen. Wir geben hierfür das folgende Beispiel.

Beispiel 1.2. Ist $\rho \in F_{(3)}$ und $p \neq 3$, dann gilt

$$
f \equiv \prod_{\substack{1 \leqslant k<l \leqslant n \\ m \leqslant l}}\left(\left(x_{k}, x_{l}\right), x_{m}\right)^{p-\varepsilon_{(l, k, m)}(f)} \prod_{1 \leqslant k<l \leqslant n}\left(\left(x_{k}, x_{l}\right), x_{l}\right)^{\varepsilon_{(k, l, l)}(f)} \bmod F_{(4)} .
$$




\section{2-Erweiterungen mit beschränkter Verzweigung}

In diesem Abschnitt studieren wir ein Beispiel für ein Dreifach-Masseyprodukt aus der Zahlentheorie. Zu diesem Zwecke betrachten wir die Relationenstruktur der maximalen außerhalb einer Primstellenmenge $S$ unverzweigten 2-Erweiterung von $\mathbb{Q}$.

Sei $S=\left\{l_{1}, \ldots, l_{n}, \infty\right\}$, wobei die $l_{i}$ ungerade Primzahlen seien. Wir bezeichnen die maximale außerhalb von $S$ unverzweigte 2-Erweiterung von $\mathbb{Q}$ mit $\mathbb{Q}_{S}(2)$, und wir setzen $G_{S}(2)=G\left(\mathbb{Q}_{S}(2) / \mathbb{Q}\right)$. Ein Ergebnis von Koch beschreibt die Struktur von $G_{S}(2)$ durch Erzeugende und Relationen. Dazu fixieren wir Fortsetzungen $L_{i}$ von $l_{i}$ nach $\mathbb{Q}_{S}(2)$. Für $i=1, \ldots, n$ bezeichne $\sigma_{i} \in G_{S}(2)$ einen Lift des Frobenius von $L_{i}$ (der zusätzlich einer gewissen klassenkörpertheoretischen Bedingung genügen muß) und $\tau_{i} \in G_{S}(2)$ einen Erzeuger der Trägheitsgruppe von $L_{i}$ (auch hier ist noch eine Normierung erforderlich). Dann gilt der folgende

Satz 2.1 (Koch, [Koc78]). Es gibt eine minimale Darstellung

$$
1 \longrightarrow R \longrightarrow F \stackrel{\pi}{\longrightarrow} G_{S}(2) \longrightarrow 1
$$

von $G_{S}(2)$, wobei $F$ eine freie pro-2-Gruppe auf Erzeugern $x_{1}, \ldots, x_{n}$ ist und die Abbildung $\pi$ durch $\pi\left(x_{i}\right)=\tau_{i}$ für $i=1, \ldots, n$ gegeben ist. Ein minimales System von Erzeugern von $R$ als Normalteiler ist durch $\rho_{1}, \ldots, \rho_{n}$ gegeben, mit

$$
\rho_{m}=x_{m}^{l_{m}-1}\left(x_{m}^{-1}, y_{m}^{-1}\right) .
$$

Dabei ist $y_{m} \in F$ ein Urbild von $\sigma_{m}$. Es gilt

$$
\rho_{m} \equiv x_{m}^{l_{m}-1} \prod_{j \neq m}\left(x_{m}, x_{j}\right)^{\ell_{m, j}} \bmod F_{(3)},
$$

mit

$$
(-1)^{\ell_{m, j}}=\left(\frac{l_{m}}{l_{j}}\right) .
$$

Wir betrachten den Fall, daß die Relationengruppe $R$ in $F_{(3)}$ liegt. Im ersten Abschnitt haben wir gesehen, wie eng in dieser Situation die Beschreibung von Elementen aus $R$ modulo $F_{(4)}$ mit Dreifach-Masseyprodukten zusammenhängt. Eine arithmetische Interpretation der Paarungen

$$
H^{1}\left(G_{S}(2)\right) \times H^{1}\left(G_{S}(2)\right) \times H^{1}\left(G_{S}(2)\right) \stackrel{\langle, \cdot, \cdot, \cdot\rangle}{\rightarrow} H^{2}\left(G_{S}(2)\right) \stackrel{\operatorname{tr}_{*}}{\rightarrow} \mathbb{Z} / 2 \mathbb{Z}
$$

(hierbei sei $\left.H^{i}\left(G_{S}(2)\right)=H^{i}\left(G_{S}(2), \mathbb{Z} / 2 \mathbb{Z}\right)\right)$ wird durch das Rédeisymbol gegeben. Dieses wurde in den dreißiger Jahren des letzten Jahrhunderts von Rédei 
eingeführt ([Réd34]) und ist wie folgt definiert. Wir betrachten Primzahlen $p_{1}, p_{2}, p_{3}$ mit $p_{i} \equiv 1 \bmod 4$, und

$$
\left(\frac{p_{1}}{p_{2}}\right)=\left(\frac{p_{1}}{p_{3}}\right)=\left(\frac{p_{2}}{p_{3}}\right)=1 .
$$

Es sei $\alpha=x+y \sqrt{p_{1}}$, wobei $x, y \in \mathbb{Z}$ Lösungen von

$$
x^{2}-p_{1} y^{2}-p_{2} z^{2}=0
$$

seien, die noch den Nebenbedingungen $\operatorname{ggT}(x, y, z)=1,2 \mid y$ und $x-y \equiv 1$ mod 4 genügen sollen. Dann existiert ein Primideal $\mathfrak{p}_{3}$ in $k_{1}=\mathbb{Q}\left(\sqrt{p_{1}}\right)$ über $p_{3}$, so daß $\mathfrak{p}_{3}$ unverzweigt in $k_{1}(\sqrt{\alpha})$ ist, und wir definieren das Rédeisymbol $\left[p_{1}, p_{2}, p_{3}\right]$ durch

$$
\left[p_{1}, p_{2}, p_{3}\right]=\left\{\begin{aligned}
1, & \text { falls } \mathfrak{p}_{3} \text { in } k_{1}(\sqrt{\alpha}) \text { zerfällt } \\
-1, & \text { falls } \mathfrak{p}_{3} \text { in } k_{1}(\sqrt{\alpha}) \text { träge ist. }
\end{aligned}\right.
$$

Das Rédeisymbol ist unabhängig von den getroffenen Wahlen ([Réd34]). Es gilt der folgende Satz (siehe [Vog]).

Satz 2.2. Sei $S=\left\{l_{1}, \ldots, l_{n}, \infty\right\}$ mit $l_{i} \equiv 1 \bmod 4, i=1, \ldots, n$, und gelte

$$
\left(\frac{l_{i}}{l_{j}}\right)=1 \text { für alle } 1 \leqslant i, j \leqslant n, i \neq j .
$$

Sei $1 \leqslant i, j, k \leqslant n$. Für $m=1, \ldots, n$ gilt

$$
\rho_{m} \equiv \prod_{\substack{1 \leqslant i<j \leqslant n \\ k<j}}\left(\left(x_{i}, x_{j}\right), x_{k}\right)^{e_{i, j, k, m}} \bmod F_{(4)}
$$

mit

$$
(-1)^{e_{i, j, k, m}}=\left\{\begin{array}{l}
{\left[l_{i}, l_{j}, l_{k}\right], \quad \begin{array}{l}
\text { falls } m=j \text { und } m \neq k, \text { oder } m \neq j \text { und } m=k \\
\text { oder } m=i \text { und } j=k, \text { oder } m=j=k \\
\text { sonst. }
\end{array}}
\end{array}\right.
$$

Bezeichnet $\chi_{1}, \ldots, \chi_{n}$ die $z u x_{1}, \ldots, x_{n}$ duale Basis von

$$
H^{1}\left(G_{S}(2)\right)=H^{1}\left(G_{S}(2), \mathbb{Z} / 2 \mathbb{Z}\right),
$$

dann gilt für das Dreifach-Masseyprodukt

$$
\langle\cdot, \cdot, \cdot\rangle: H^{1}\left(G_{S}(2)\right) \times H^{1}\left(G_{S}(2)\right) \times H^{1}\left(G_{S}(2)\right) \rightarrow H^{2}\left(G_{S}(2)\right)
$$

die folgende Identität

$(-1)^{\operatorname{tr}_{\rho_{m}}\left\langle\chi_{i}, \chi_{j}, \chi_{k}\right\rangle}=\left\{\begin{aligned} {\left[l_{i}, l_{j}, l_{k}\right] } & \text { falls } m=i \text { und } m \neq k, \text { oder } m \neq i \text { und } m=k, \\ 1 & \text { sonst. }\end{aligned}\right.$ 
Beispiel 2.3. Für $S=\{13,61,937, \infty\}$ haben wir

$$
\begin{aligned}
\rho_{1} & \equiv\left(\left(x_{2}, x_{3}\right), x_{1}\right) \bmod F_{(4)}, \\
\rho_{2} & \equiv\left(\left(x_{1}, x_{3}\right), x_{2}\right) \bmod F_{(4)}, \\
\rho_{3} & \equiv\left(\left(x_{1}, x_{3}\right), x_{2}\right)\left(\left(x_{2}, x_{3}\right), x_{1}\right) \equiv\left(\left(x_{1}, x_{2}\right), x_{3}\right) \bmod F_{(4)}
\end{aligned}
$$

Ein ähnlicher Satz wie der obige existiert auch für den 2-Klassenkörperturm imaginärquadratischer Zahlkörper, siehe [Vog]. Bezeichnet $K$ einen imaginärquadratischen Zahlkörper und $K_{\varnothing}$ seine maximale unverzweigte 2-Erweiterung, dann kann die vom Masseyprodukt und der Spurabbildung induzierte Paarung $H^{1}\left(G\left(K_{\varnothing} / K\right)\right) \times H^{1}\left(G\left(K_{\varnothing} / K\right)\right) \times H^{1}\left(G\left(K_{\varnothing} / K\right)\right) \rightarrow H^{2}\left(G\left(K_{\varnothing} / K\right)\right) \rightarrow \mathbb{Z} / 2 \mathbb{Z}$ ebenfalls durch das Rédeisymbol beschrieben werden. Insbesondere erhalten wir Paarungen

$$
(\mathrm{Cl}(K) / 2)^{*} \times(\mathrm{Cl}(K) / 2)^{*} \times(\mathrm{Cl}(K) / 2)^{*} \rightarrow \mathbb{Z} / 2 \mathbb{Z},
$$

wobei $\mathrm{Cl}(K)$ die Idealklassengruppe von $K$ und * das Pontrjagin-Dual bezeichnen.

\section{References}

[Iha86] Y. IHARA - On Galois representations arising from towers of coverings of $\mathbf{P}^{1} \backslash\{0,1, \infty\}$, Invent. Math. 86 (1986), no. 3, p. 427-459.

[Koc78] H. КоCH - Galois cohomology of algebraic number fields, appendix, VEB Deutscher Verlag der Wissenschaften, Berlin, 1978.

[Mor04] M. MoRISHITA - Milnor invariants and Massey products for prime numbers, Compos. Math. 140 (2004), no. 1, p. 69-83.

[NSW00] J. NeukiRch, A. Schmidt \& K. WingBerg - Cohomology of number fields, Grundlehren der Mathematischen Wissenschaften, vol. 323, SpringerVerlag, Berlin, 2000.

[Réd34] L. RÉDEI - Ein neues zahlentheoretisches Symbol mit Anwendungen auf die Theorie der quadratischen Zahlkörper, I, J. reine u. angew. Math. 171 (1934), p. 55-60.

[Vog] D. Vogel - On 2-extensions with restricted ramification, erscheint im $J$. reine $u$. angew. Math. 
Mathematisches Institut, Seminars, (Y. TschInkel, ed.), p. 99-108

Universität Göttingen, 2004-05

\title{
FINITE FLAT COMMUTATIVE GROUP SCHEMES OVER COMPLETE DISCRETE VALUATION FIELDS: CLASSIFICATION, STRUCTURAL RESULTS; APPLICATION TO REDUCTION OF ABELIAN VARIETIES
}

\section{M.V. Bondarko}

St. Petersburg State University, Department of Higher Algebra and Number Theory, Bibliotechnaya Pl. 1, 198904 St. Petersburg, Russia

E-mail : mbondarko@hotmail.com

\begin{abstract}
This is a summary of author's results on finite flat commutative group schemes. The properties of the generic fibre functor are discussed. A complete classification of finite local flat commutative group schemes over mixed characteristic complete discrete valuation rings in terms of their Cartier modules (defined by Oort) is given. We also state several properties of the tangent space of these schemes. These results are applied to the study of reduction of Abelian varieties. A finite $p$-adic semistable reduction criterion is formulated. It looks especially nice in the ordinary reduction case. The plans of the proofs are described.
\end{abstract}

Notation. Throughout, $K$ is a mixed characteristic complete discrete valuation field with residue field of characteristic $p, L$ is a finite extension of $K ; \mathfrak{O}_{K} \subset \mathfrak{O}_{L}$ are their rings of integers, $e$ is the absolute ramification index of $L, s=\left[\log _{p}(e /(p-1))\right], e_{0}=\left[L:\left(K_{n r} \cap L\right)\right]\left(e_{0}=e(L / K)\right.$ in the perfect residue field case), $l^{\prime}=s+v_{p}\left(e_{0}\right)+1, l=2 s+v_{p}\left(e_{0}\right)+1 ; \bar{L}$ denotes the residue field of $\mathfrak{O}_{L} ; \mathfrak{M}$ is the maximal ideal of $\mathfrak{O}_{L} ; \pi \in \mathfrak{M}$ is some uniformizing element of $L$.

A 'group scheme' will (by default) mean a finite flat commutative group scheme, $S / \mathfrak{O}_{L}$ means a finite flat commutative group scheme over $\mathfrak{O}_{L}$. For finite group schemes $S, T$ we write $S \triangleleft T$ if $S$ is a closed subgroup scheme of $T$.

November 13, 2004. 


\section{The category of finite flat commutative group schemes; the generic fibre results}

We denote by $\mathfrak{F} \mathfrak{G} \mathfrak{S}_{R}$ the category of finite flat commutative $p$-group schemes (i.e. annihilated by a power of $p$ ) over a base ring $R$.

The goal of this paper is the study of $\mathfrak{F} \mathfrak{G} \mathfrak{S}_{\mathfrak{O}_{L}}$ and of Abelian varieties over $L$. We note that a certain classification of $\mathfrak{F} \mathfrak{G S}_{\mathfrak{O}_{L}}$ for $\bar{L}$ being perfect was given by Breuil (see [Bre00]); yet that classification is inconvenient for several types of problems.

It is well-known that any finite flat group scheme over $L$ is étale; hence $\mathfrak{F} \mathfrak{G} \mathfrak{S}_{L}$ is equivalent to the category of finite modules over the absolute Galois group of $L$. In particular, this category is abelian. Hence it is natural to consider the generic fibre functor

$$
G F: S \rightarrow S_{L}=S \times_{\operatorname{Spec} \mathfrak{O}_{L}} \operatorname{Spec} L .
$$

The functor $G F$ is faithful and defines a one-to-one correspondence between closed subgroup schemes of $S$ and closed subgroup schemes of $S_{L}$ (see [Ray74]).

It was also proved by Raynaud in the case $e<p-1$ that $G F$ is full; besides $\mathfrak{F} \mathfrak{G} \mathfrak{S}_{\mathfrak{O}_{L}}$ is an Abelian category. Neither of this facts is true for larger values of $e$. Moreover, one cannot apply Raynaud's methods in the case $e \geq p-1$.

Yet the following important result is valid.

Theorem 1.1. If $S, T / \mathfrak{O}_{L}$ are group schemes, $g: S_{L} \rightarrow T_{L}$ is an L-group scheme morphism, then there exists an $h: S \rightarrow T$ over $\mathfrak{O}_{L}$ such that $h_{L}=p^{s} g$.

Note that $s=0$ for $e<p-1$; therefore Theorem 1.1 generalizes the fullness result of Raynaud. Hence $G F$ is 'almost full'. One easily checks that the result is sharp, i.e. the value of $s$ its the best possible.

Theorem 1.1 also can be considered as a finite analogue of fullness of the generic fibre functor for $p$-divisible groups (proved by Tate). Besides, it implies Tate's result (see [Tat67]) immediately.

The main tool of the proof is the Cartier module functor for finite local group schemes. It will be defined below. A similar statement for Ext $^{1}$ follows easily from Theorem 1.1 and the Cartier module theory for group schemes.

\section{Formal groups; Cartier modules}

Our basic method is resolving finite group schemes by means of $p$-divisible groups (in particular, by finite height formal group laws). We recall the Cartier module theory for formal group laws. Here we describe a modified version that was used in [BV03] and [V.05] (cf. [Haz78] and [Zin84]). 
We denote by $C$ the category of additive subgroups of $L[[\Delta]]^{m}, m>0$. For $C_{1}, C_{2} \in C, \operatorname{dim} C_{i}=m_{i}$ we define

$$
C\left(C_{1}, C_{2}\right)=A \in M_{m_{2} \times m_{1}} \mathfrak{O}_{K}: A C_{1} \subset C_{2} .
$$

For $h \in(L[[\Delta]])^{m}$, the coefficients of $h$ are equal to $h_{i}=\sum_{l \geq 0} c_{i l} \Delta^{l}, c_{i l} \in L$, we define

$$
h(x)=\left(h_{i}(x)\right), 1 \leq i \leq m, \text { where } h_{i}(x)=\sum_{l \geq 0} c_{i l} x^{p^{l}} .
$$

For an $m$-dimensional formal group law $F / \mathfrak{O}_{L}$ we consider

$$
D_{F}=\left\{f \in L[[\Delta]]^{m}: \exp _{F}(f(x)) \in \mathfrak{O}_{L}[[x]]^{m}\right\},
$$

where $\exp _{F} \in L[[X]]^{m}$ is the composition inverse to the logarithm of $F$. In particular, for $m=1$ we have $\sum a_{i} \Delta^{i} \in D_{F} \Longleftrightarrow \exp _{F}\left(\sum a_{i} x^{p^{i}}\right) \in \mathfrak{O}_{L}[[x]]$.

Then the Cartier theory easily implies the following fact.

\section{Proposition 2.1.}

1. $F \rightarrow D_{F}$ defines a full embedding of the category of formal groups over $\mathfrak{O}_{L}$ into $C$.

If $f: F_{1} \rightarrow F_{2}, f \equiv A X \bmod \operatorname{deg} 2, A \in M_{m_{2} \times m_{1}} \mathfrak{O}_{L}$, then the associated map $f_{*}: D_{F_{1}} \rightarrow D_{F_{2}}$ is the multiplication by $A$.

2. $D_{F_{1}}=D_{F_{2}}$ if and only if the groups $F_{1}$ and $F_{2}$ are strictly isomorphic, i.e. there exists an isomorphism whose Jacobian is the identity matrix.

Now we briefly recall the notion of the Cartier ring. For a commutative ring $Q$ and a $Q$-algebra $P$ one can introduce the following operators on $P[[\Delta]]$. For $f=\sum_{i \geq 0} c_{i} \Delta^{i} \in P[[\Delta]], a \in Q$ we define

$$
\mathbf{V} f=f \Delta ; \mathbf{f} f=\sum_{i>0} p c_{i} \Delta^{i-1} ;\langle a\rangle f=\sum a^{p^{i}} c_{i} \Delta^{i}
$$

$\operatorname{Cart}(Q)$ (the Cartier $p$-ring, see $[\mathbf{H a z 7 8}]$ ) is the ring that is generated by $\mathbf{V}, \mathbf{f},\langle a\rangle, a \in Q$ and factorized modulo certain natural relations (see [Haz78], Sect. 16.2, [M.V04a], [M.V04b], [M.V04c]). If $M$ is a $\operatorname{Cart}(Q)$-module, then $M / \mathbf{V} M$ has a natural structure of a $Q$-module defined via $a \cdot(x \bmod \mathbf{V} M)=$ $\langle a\rangle x \bmod \mathbf{V} M$ for any $x \in M$. We introduce an important definition (see [M.V04a]).

\section{Definition 2.2.}

1. For Cart-modules $M \subset N$ we write $M \triangleleft N$, if for any $x \in M, \mathbf{V} x \in N$, we have $x \in M$. We call $M$ a closed submodule of $N$.

2. Cart-module $N$ is called separated if $\{0\} \triangleleft N$, i.e. $N$ has no $\mathbf{V}$-torsion. 
We denote $\operatorname{Cart}\left(\mathfrak{O}_{L}\right)$ by Cart; we call Cart-modules Cartier modules. Note that we don't define closed subsets of Cartier modules. Yet we could define a topology on any Cart-module $M$ whose closed subsets would be $C \subset M$ : $\mathbf{V} x \in C \Longrightarrow x \in C$. Then any Cart-map would be continuous.

We define the closure of a subset $N$ of a Cartier module $M$ as the smallest closed Cart-submodule of $M$ that contains $N$.

\section{Proposition 2.3.}

1. For any $F / \mathfrak{O}_{L}$ the group $D_{F}$ is a Cart-module via the action of operators defined above; it is canonically Cart-isomorphic to the module of p-typical curves for $F$ (see [Haz78] and [Zin84]).

2. $C\left(D_{F_{1}}, D_{F_{2}}\right)=\operatorname{Cart}\left(D_{F_{1}}, D_{F_{2}}\right)$.

3. $M \in C$ is equal to $D_{F}$ for some $m$-dimensional $F / \mathfrak{O}_{L}$ iff $C \triangleleft L[[\Delta]]^{m}$ and $C \bmod \Delta=\mathfrak{O}_{L}{ }^{m}$.

In the papers [V.05] and [BV03] two functors on the category of formal groups were defined. The first (called the fraction part) was similar to those defined by Grothendieck, Messing and Fontaine; yet it was defined in a quite different way and was described more precisely than the functor in the book [Fon77]. The behaviour of the fraction part is (in some sense) linear.

The second functor (denoted by $M_{F}$ ) described the obstacle for the Fontaine's functor to be an embedding of categories. For a finite height formal group $F$ the value of $M_{F}$ can be described by means of $D_{F_{\pi}}$. Here $F_{\pi}=\pi^{-1}(\pi X, \pi Y)$. Since the coefficients of $F_{\pi}$ tend to 0 quickly, the obstacle functor is 'finite'. One may say that its complexity is killed by $\mathbf{f}^{s}$ (see Proposition 3.2 .2 of $[\mathbf{M . V 0 4 a}])$.

\section{Cartier-Oort modules of local group schemes}

Let $S$ be a local group scheme over $\mathfrak{O}_{L}$; let $0 \rightarrow S \rightarrow F \rightarrow G \rightarrow 0$ be its resolution by means of finite height formal groups. We define $C(S)=$ $\operatorname{Coker}\left(D_{F} \rightarrow D_{G}\right)$. In the paper [Oor74] it was proved that $S \rightarrow C(S)$ is a well-defined functor on the category of local (finite flat commutative) group schemes over $\mathfrak{O}_{L}$; it defines an embedding of this category into the category of Cart-modules. We call $C(S)$ the Oort module of $S$. The theory of Oort also can be used when the base ring is a field of characteristic $p$. In this case f corresponds to the Frobenius, $\mathbf{V}$ corresponds to the Verschiebung operator (see $[$ Zin84]).

Now we state the main classification result. It completely describes the properties of the Oort functor. 


\section{Theorem 3.1.}

I 1. Closed submodules of $C(S)$ are in one-to-one correspondence with closed subgroup schemes of $S$.

2. If $M=C(S), N=C(H) \triangleleft M$, where $H \triangleleft S$, then $M / N \approx C(S / H)$.

3. Conversely, exact sequences (as fppf-sheaves, i.e. the inclusion is a closed embedding) of local schemes induce exact sequences of Oort modules.

II If $f: S \rightarrow T$ is a local group scheme morphism, then $\operatorname{Ker} f_{*}=C(\operatorname{Ker} f)$, where $f_{*}$ is the induced Oort modules homomorphism; we consider the kernel in the category of flat group schemes.

III $A$ Cart-module $M$ is isomorphic to $C(S)$ for $S$ being a finite flat commutative local group scheme over $\mathfrak{O}_{L}$ if and only if $M$ satisfies the following conditions.

1. $M / \mathbf{V} M$ is a finite length $\mathfrak{O}_{L}$-module.

2. $M$ is separated.

3. $\cap_{i \geq 0} \mathbf{V}^{i} M=\{0\}$.

4. $M=\mathrm{Cl}_{M}(\langle\pi\rangle M)$.

IV The minimal dimension of a finite height formal group $F$ such that $S$ can be embedded into $F$ is equal to $\operatorname{dim}_{\mathfrak{O}_{L}}(C(S) / \mathbf{V} C(S)$ ) (i.e. to the number of indecomposable $\mathfrak{O}_{L}$-summands of $\left.C(S) / \mathbf{V} C(S)\right)$.

$\mathrm{V} M=C\left(\operatorname{Ker}\left[p^{r}\right]_{F}\right)$ for an $m$-dimensional formal group $F$ if and only if in addition to the conditions of III, $p^{r} M=0$ and $M / \mathbf{V} M \approx\left(\mathfrak{O}_{L} / p^{r} \mathfrak{O}_{L}\right)^{m}$.

VI If $S, T$ are local, then $\operatorname{Ext}^{1}(S, T)=\operatorname{Ext}_{\text {Cart }}^{1}(C(S), C(T))$. Here we consider extensions in the category of finite flat group schemes, whence the definition of an exact sequence is the same as always.

We introduce a natural definition of the tangent space $T S$ for a finite group scheme $S$.

Definition 3.2. For a finite flat groups scheme $S$ we denote by $T S$ the $\mathfrak{O}_{L^{-}}$ dual of $J / J^{2}$ (i.e. $\operatorname{Hom}_{\mathfrak{O}_{L}}\left(J / J^{2}, L / \mathfrak{O}_{L}\right)$ ), where $J$ is the augmentation ideal of the affine algebra of $S$.

It is well-known that the tangent space of a group scheme is equal (i.e. naturally isomorphic) to the tangent space of its local part. Besides, if $P$ is any (unitial commutative) $\mathfrak{O}_{L}$-algebra then the (suitably defined) tangent space of $S_{P}=S \times \operatorname{Spec} \mathfrak{O}_{L} \operatorname{Spec} P$ is canonically isomorphic to $T S \otimes_{\mathfrak{O}_{L}} P$.

We state the main properties of the tangent space functor. 


\section{Theorem 3.3.}

I TS is naturally isomorphic to $C\left(S_{0}\right) / \mathbf{V C}\left(S_{0}\right)$, where $S_{0}$ is the local part of $S$.

II $f: S \rightarrow T$ is a closed embedding of local group schemes if and only if the induced map on the tangent spaces is an embedding.

III If $0 \rightarrow H \rightarrow S \rightarrow T \rightarrow 0$ is an exact sequence of local group schemes (in the category of fppf-sheaves, i.e. $H \triangleleft S$ ) then the corresponding sequence of tangent spaces is also exact.

IV For a local group scheme $S$ the following numbers are equal.

1. The $\mathfrak{O}_{L}$-dimension of $\mathrm{J} / \mathrm{J}^{2}$.

2. The $\mathfrak{O}_{L}$-dimension of $C(S) / \mathbf{V C}(S)$.

3. The minimal dimension of a finite height formal group $F$ such that $S \triangleleft F$.

$\mathrm{V}$ A local group scheme $S$ is equal to $\operatorname{Ker}\left[p^{r}\right]_{F}$ for some $m$-dimensional finite height formal group $F / \mathfrak{O}_{L}$ if and only if $p^{r} S=0$, and $T S \approx\left(\mathfrak{O}_{L} / p^{r} \mathfrak{O}_{L}\right)^{m}$.

\section{Finite criteria for reduction of Abelian varieties}

As an application of the results on finite group schemes certain finite $p$-adic criteria for semistable and ordinary reduction of Abelian varieties were proved. We call these criteria finite because in contrast to Grothendieck's criteria (see [Gro72]) it is sufficient to check certain conditions on some finite $p$-torsion subgroups of $V$ (instead of the whole $p$-torsion).

We recall that an Abelian variety (over $\mathfrak{O}_{K}$ or $\mathfrak{O}_{L}$ ) is called an ordinary reduction one (or just ordinary) if the connected component of 0 of the reduction of the Néron model of $A$ is an extension of a torus by an ordinary Abelian variety (over $\bar{L}$ ). In particular, an ordinary variety has semistable reduction. For example, a semistable reduction elliptic curve is either ordinary or supersingular.

Let $V$ be be an Abelian variety of dimension $m$ over $K$ that has semistable reduction over $L$.

\section{Theorem 4.1.}

I $V$ has semistable reduction over $K$ if and only if for there exists a finite flat group scheme $H / \mathfrak{O}_{K}$ such that $T H_{\mathfrak{O}_{L}} \supset\left(\mathfrak{O}_{L} / p^{l} \mathfrak{O}_{L}\right)^{m}$ (i.e. there exists an embedding) and a monomorphism $g: H_{K} \rightarrow \operatorname{Ker}\left[p^{l}\right]_{V, K}$. 
II $V$ has ordinary reduction over $K$ if and only if for some $H_{K} \subset \operatorname{Ker}\left[p^{l}\right]_{V, K}$ and $M$ unramified over $K$ we have $H_{M} \cong\left(\mu_{p^{l}, M}\right)^{m}$. Here $\mu$ denotes the group scheme of roots of unity.

Part I is a generalisation of Theorem 5.3 of [Con99] where the case $e<p-1$, $V$ of good reduction over $\mathfrak{O}_{L}$, was considered.

Finite $l$-adic criteria (see $[\mathbf{S Z 0 0}]$ ) seem to be easier to use; yet they don't allow to check whether the reduction is ordinary.

If the reduction of $A$ over $L$ is good then $l$ can be replaced by $l^{\prime}$.

\section{Ideas of proofs}

Proposition 2.3 is an easy consequence of the usual Cartier theory. It easily implies parts I and II of Theorem 3.1, and parts I - III of Theorem 3.3. The proof the necessity of conditions of part III in Theorem 3.1 is also more-or-less easy.

To prove sufficiency of conditions of part III in Theorem 3.1 one applies the explicit description of the Cartier module of a formal group (see Section 27.7 of $[\mathbf{H a z 7 8}]$ ) and constructs a formal group $F$ such that $M$ is a Cart-factor of $D_{F}$. A formal group of dimension $\operatorname{dim}_{\mathfrak{O}_{L}}(M / \mathbf{V} M)$ can be chosen. Next one proves that a finite height formal group can be chosen. In this case $M$ will be equal to $D_{F} / N$ for some $N \triangleleft D_{F}$ such that $N$ is Cart-isomorphic to $D_{G}$ for a finite height formal group $G$. Lastly one verifies that $M=C(S)$ for $S$ being the kernel of a certain isogeny $h: G \rightarrow F$. Under the conditions of part $\mathrm{V}$ of Theorem 3.1 (and Theorem 3.3) one obtains that $G \approx F$, and $h=\operatorname{Ker}\left[p^{r}\right]_{F}$.

Parts IV and V of Theorem 3.3 are reformulations of the corresponding parts of Theorem 3.1 in terms of tangent spaces. Part VI of Theorem 3.1 follows from the fact the the conditions of part III are preserved by extensions.

Now we sketch the proof of Theorem 1.1. First the following important results on the reductions of group schemes are proved.

\section{Proposition 5.1.}

1. If the map $h: S \rightarrow T$ of $\mathfrak{O}_{L}$-group schemes is injective on the generic fibre, then the kernel of the reduction map (as a kernel of a group scheme morphism over $\bar{L}$ ) is annihilated by $\mathbf{f}^{s}$.

2. If the map $h: S \rightarrow T$ of $\mathfrak{O}_{L}$-group schemes is surjective on the generic fibre, then the cokernel of the reduction map is annihilated by $\mathbf{V}^{s}$.

In the imperfect residue field case we extend $\bar{L}$ so that $\mathrm{Fr}^{-s}($ Coker $\bar{h})$ will be defined over $\bar{L}$. 
Part 1 is proved by an analysis of the properties of the 'obstacle' functor (see the end of $\S 2$ ); part 2 follows immediately as the dual of part 1 .

In the proof of Proposition 5.1 (and in several other places) the local-étale exact sequence for finite flat commutative group schemes is used to reduce the problem to the case of local group schemes (and hence to formal groups). Some of these reductions are quite complicated.

Next one proceeds to the proof of Theorem 1.1 using the fact $\mathbf{f}^{s} \mathbf{V}^{s}=p^{s}$.

For the proofs of reduction criteria explicit Cartier module descent is used.

In $[\mathbf{M . V 0 4 b}]$ the following lemmas were were proved. For the first an explicit descent reasoning for $D_{F}$ was used; the second is an easy flat descent exercise.

\section{Proposition 5.2.}

1. Let $F$ be a finite height formal group over $\mathfrak{O}_{L}$. Suppose that its generic fibre (as a p-divisible group) $F_{L}=F \times_{\operatorname{Spec} \mathfrak{O}_{L}} \operatorname{Spec} L$ is defined over $K$, i.e. there exists a p-divisible group $Z_{K}$ over $K$ such that

$$
Z_{K} \times_{\text {Spec } K} \operatorname{Spec} L \cong F_{L} \text {. }
$$

Suppose that for $t=v_{p}\left(e_{0}\right)+1$ and some group scheme $T / \mathfrak{O}_{K}$ we have $\operatorname{Ker}\left[p^{t}\right]_{Z} \approx T \times_{\operatorname{Spec} \mathfrak{O}_{K}} \operatorname{Spec} K$. Suppose also that this isomorphism combined with the isomorphism (2) is the generic fibre of a certain isomorphism $T \times_{\text {Spec } \mathfrak{O}_{K}} \operatorname{Spec} \mathfrak{O}_{L} \cong \operatorname{Ker}\left[p^{t}\right]_{F}$. Then $Z_{K} \approx F_{K}^{\prime}$ for some formal group $F^{\prime} / \mathfrak{O}_{K}$.

2. Let $V$ be a p-divisible group over $\mathfrak{O}_{L}$. Suppose that its generic fibre is defined over $K$. Then $V$ is defined over $\mathfrak{O}_{K}$ if and only if its local part is.

Using this, Theorem 1.1, and Cartier modules of group schemes one can prove a certain good reduction criterion for Abelian varieties (see [M.V04b]). We don't formulate that criterion here.

Using Proposition 5.2 and a certain tangent space argument one proves the following fact.

Proposition 5.3. Let $V$ be a p-divisible group over $K$, let $Y$ be a p-divisible group of dimension $m$ over $\mathfrak{O}_{L}$ (i.e. its local part is a formal group of dimension $m$ ). Suppose that $V \times_{\operatorname{Spec} K} \operatorname{Spec} L \cong Y \times_{\operatorname{Spec} \mathfrak{O}_{L}} \operatorname{Spec} L$. The following conditions are equivalent:

I There exists a p-divisible group $Z$ over $\mathfrak{O}_{K}$ such that $V \cong Z \times_{\operatorname{Spec}} \mathfrak{O}_{K}$ $\operatorname{Spec} K$.

II For some (finite flat commutative) group scheme $H / \mathfrak{O}_{K}$ we have $T H_{\mathfrak{O}_{L}} \approx$ $\left(\mathfrak{O}_{L} / p^{l^{\prime}} \mathfrak{O}_{L}\right)^{m}$ and there exists a monomorphism $g: H_{K} \rightarrow \operatorname{Ker}\left[p^{l^{\prime}}\right]_{V, K}$.

III We have $T H_{\mathfrak{O}_{L}} \supset\left(\mathfrak{O}_{L} / p^{l^{\prime}} \mathfrak{O}_{L}\right)^{m}$ (i.e. there exists an embedding); there exists a monomorphism $g: H_{K} \rightarrow \operatorname{Ker}\left[p^{l^{\prime}}\right]_{V, K}$. 
Note that II $\Longrightarrow$ III is obvious; I $\Longrightarrow$ II follows immediately from part V of Theorem 3.3.

Now we sketch the proof of Theorem 4.1. Let $Y_{L}$ denote the finite part of $T_{p}(V)$ (the $p$-torsion of $V$ ) considered as a $p$-divisible group over $L$.

If $V$ has semistable reduction over $K$ then $Y_{L}$ corresponds to a certain $m$-dimensional $p$-divisible group $Y$ defined over $\mathfrak{O}_{K}$. Therefore we can take $H=\left[\operatorname{Ker} p^{l}\right]_{Y, \mathcal{O}_{K}}$. It will be multiplicative if $V$ is ordinary.

For the converse implication a certain tangent space argument along with Proposition 5.3 proves that $Y_{L}$ is defined over $\mathfrak{O}_{K}$. Dualizing, we obtain that $T_{p}(V)$ is Barsotti-Tate of echelon 2 over $\mathfrak{O}_{K}$. Then Proposition $5.13 \mathrm{c}$ of $[$ Gro72] proves part I.

Lastly it remains to note that a $p$-divisible group $Y$ over $\mathfrak{O}_{L}$ is multiplicative iff $\operatorname{Ker}[p]_{Y}$ is. Then an easy tangent space calculation proves part II.

\section{References}

[Bre00] C. Breuil - Groupes p-divisibles, groupes finis et modules filtrés, Ann. of Math. (2) 152 (2000), no. 2, p. 489-549.

[BV03] M. V. Bondarko \& S. V. Vostokov - Explicit classification of formal groups over local fields, Tr. Mat. Inst. Steklova 241 (2003), no. Teor. Chisel, Algebra i Algebr. Geom., p. 43-67.

[Con99] B. ConRAD - Finite group schemes over bases with low ramification, Compositio Math. 119 (1999), no. 3, p. 239-320.

[Fon77] J.-M. FonTAINE - Groupes p-divisibles sur les corps locaux, Société Mathématique de France, Paris, 1977, Astérisque, No. 47-48.

[Gro72] A. GROTHENDIECK - Groupes de monodromie en géométrie algébrique. I, Springer-Verlag, Berlin, 1972, Séminaire de Géométrie Algébrique du BoisMarie 1967-1969 (SGA 7 I), Avec la collaboration de M. Raynaud et D. S. Rim, Lecture Notes in Mathematics, Vol. 288.

[Haz78] M. Hazewinkel - Formal groups and applications, Pure and Applied Mathematics, vol. 78, Academic Press Inc., New York, 1978.

[M.V04a] B. M.V. - Finite flat commutative group schemes over complete discrete valuation fields I: the generic fibre functor, 2004, (Russian).

[M.V04b] _ Finite flat commutative group schemes over complete discrete valuation fields II: good reduction of Abelian varieties, 2004, (Russian).

[M.V04c] _ Finite flat commutative group schemes over complete discrete valuation rings III: classification, tangent spaces, and semistable reduction of Abelian varieties, 2004, Göttingen.

[Oor74] F. OorT - Dieudonné modules of finite local group schemes, Nederl. Akad. Wetensch. Proc. Ser. A 77=Indag. Math. 36 (1974), p. 284-292. 
[Ray74] M. Raynaud - Schémas en groupes de type $(p, \ldots, p)$, Bull. Soc. Math. France 102 (1974), p. 241-280.

[SZ00] A. Silverberg \& Y. G. ZARHin - Reduction of abelian varieties, in The arithmetic and geometry of algebraic cycles (Banff, AB, 1998), NATO Sci. Ser. C Math. Phys. Sci., vol. 548, Kluwer Acad. Publ., Dordrecht, 2000, p. 495-513.

[Tat67] J. T. TATE - p-divisible groups., in Proc. Conf. Local Fields (Driebergen, 1966), Springer, Berlin, 1967, p. 158-183.

[V.05] B. M. V. - Explicit classification of formal groups over complete discrete valuation fields with imperfect residue field, 2005, (Russian), to appear in Trudy St. Peterburgskogo Matematicheskogo Obsh'estva, vol. 11.

[Zin84] T. ZINK - Cartiertheorie kommutativer formaler Gruppen, Teubner-Texte zur Mathematik, vol. 68, BSB B. G. Teubner Verlagsgesellschaft, Leipzig, 1984 . 
Mathematisches Institut, Seminars, (Y. Tschinkel, ed.), p. 109-115

Universität Göttingen, 2004-05

\title{
TORIC REDUCTION AND TROPICAL GEOMETRY
}

\section{A. Szenes}

ME Institute of Mathematics, Geometry Department, Egry József u. 1, H Ép., H-1111 Budapest, Hungary • E-mail : szenes@math.bme.hu

\begin{abstract}
In this note, I review a result obtained in joint work with Michèle Vergne on a duality principle in toric geometry. I will demonstrate the essential points of our work on a concrete example.
\end{abstract}

\section{Toric varieties and quotients}

The basic data is a real vector space $\mathfrak{g}$ endowed with a basis $\left[\omega_{1}, \omega_{2}, \ldots, \omega_{n}\right]$, and an exact sequence

$$
0 \longrightarrow \mathfrak{a} \stackrel{\iota}{\longrightarrow} \mathfrak{g} \stackrel{\pi}{\longrightarrow} \mathfrak{t} \longrightarrow 0
$$

of real vector spaces of dimensions $r, n$ and $d$, such that the three lattices

$$
\mathfrak{g}_{\mathbb{Z}}=\oplus_{i=1}^{n} \mathbb{Z} \omega_{i}, \mathfrak{t}_{\mathbb{Z}}=\pi\left(\mathfrak{g}_{\mathbb{Z}}\right), \mathfrak{a}_{\mathbb{Z}}=\operatorname{ker}\left(\left.\pi\right|_{\mathfrak{g}_{\mathbb{Z}}}\right) .
$$

have maximal rank in the corresponding vector spaces. Clearly, we have $n-d=$ $r$. This construction gives rise to two sequences of vectors

$$
\mathfrak{A}=\left[\alpha_{1}, \ldots, \alpha_{n}\right] \subset \mathfrak{a}_{\mathbb{Z}}^{*}, \quad \alpha_{i}=\iota^{*}\left(\omega^{i}\right)
$$

where $\omega^{i}$ is the dual basis in $\mathfrak{g}^{*}$, and

$$
\mathfrak{B}=\left[\beta_{1}, \ldots, \beta_{n}\right] \subset \mathfrak{t}_{\mathbb{Z}}, \quad \beta_{i}=\pi\left(\omega_{i}\right)
$$

One says that $\mathfrak{A}$ and $\mathfrak{B}$ are Gale dual to each other in this situation.

November 4, 2004. 
We will assume that $\mathfrak{A}$ is projective, i.e. there is $\gamma \in \mathfrak{a}$ such that $\left\langle\alpha_{i}, \gamma\right\rangle>0$ for $i=1, \ldots, n$. This is equivalent to the condition that the origin is contained in the convex hull of $\mathfrak{B}$.

We can write down our maps as integer matrices $[\iota]$ and $[\pi]$ if we choose bases of $\mathfrak{a}_{\mathbb{Z}}$ and $\mathfrak{t}_{\mathbb{Z}}$. Then the set of $\alpha_{i}$ s are the column vectors of $[\iota]$ and the $\beta_{i}$ s are the row vectors of $[\pi]$.

It is a pleasant exercise to check that a pair of matrices $[\iota]$ and $[\pi]$ with integer coefficients represents an exact sequence as described above if the g.c.d of the $r$-by- $r$ minors of $[\iota]$ is 1 , the same condition holds for $[\pi]$, and

$$
[\iota] \cdot[\pi]=0
$$

We will consider the following example of this setup:

$$
\left[\iota_{0}\right]=\left(\begin{array}{llll}
0 & 1 & 0 & 1 \\
1 & 1 & 1 & 0
\end{array}\right) \quad\left[\pi_{0}\right]=\left(\begin{array}{cc}
0 & 1 \\
-1 & 0 \\
1 & -1 \\
1 & 0
\end{array}\right)
$$

Denoting the coordinates on $\mathfrak{a}$ by $x$ and $y$, we can list the $\alpha_{i}$ s as follows:

$$
\mathfrak{A}_{0}=\left[\alpha_{1}=y, \alpha_{2}=x+y, \alpha_{3}=y, \alpha_{4}=x\right]
$$

The set $\mathfrak{B}_{0}$ may be read off the matrix $\left[\pi_{0}\right]$. We obtain the following pictures:
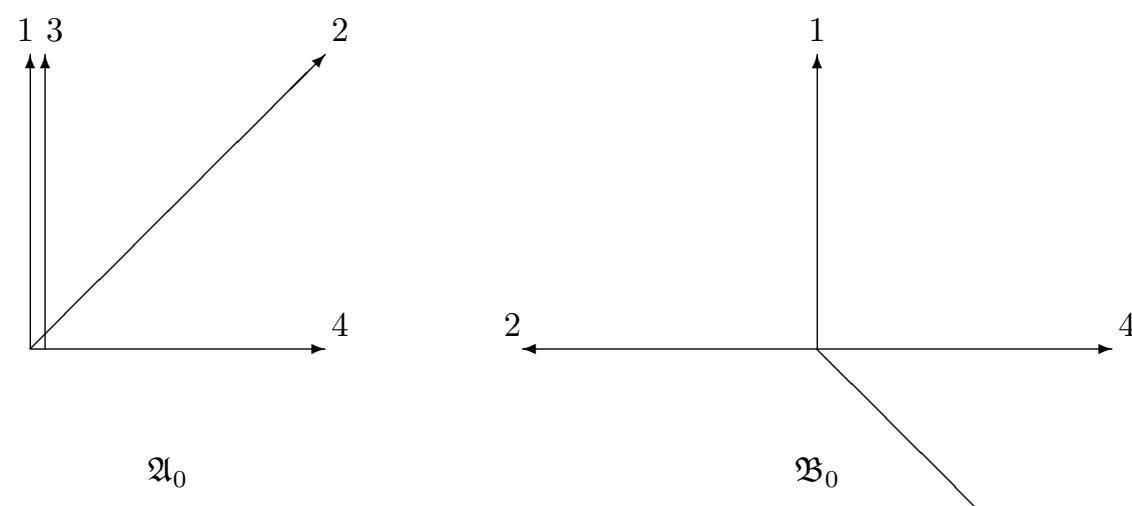

$\mathfrak{A}_{0}$ $\mathfrak{B}_{0}$ 
Next we introduce the set of $\operatorname{Bases}(\mathfrak{A})$ as those subsets $\sigma \in[1, n]$ for which $\left\{\alpha_{i} ; i \in \sigma\right\}$ is a basis of $\mathfrak{a}^{*}$; we used the notation $[1, n]=\{1, \ldots, n\}$ here. The set of chambers $\mathfrak{C}(\mathfrak{A})$, by definition, is the set of connected components the open subset

$$
\sum_{i=1}^{n} \mathbb{R}^{>0} \alpha_{i} \backslash \bigcup_{\sigma \in \operatorname{Bases}(\mathfrak{A})} \partial\left(\sum_{i \in \sigma} \mathbb{R}^{>0} \alpha_{i}\right),
$$

which is thus the complement of the boundaries of $r$-dimensional simplicial cones spanned by the vectors from $\mathfrak{A}$. In our example $\left|\operatorname{Bases}\left(\mathfrak{A}_{0}\right)\right|=5$ and $\left|\mathfrak{C}\left(\mathfrak{A}_{0}\right)\right|=2$.

The following statement describes the Gale dual picture:

Proposition 1.1. There is a one-to-one correspondence between the chambers $\mathfrak{C}(\mathfrak{A})$ and complete simplicial fans in $\mathfrak{t}$ whose one-dimensional faces form a subset of $\mathfrak{B}$.

One can associate a projective orbifold toric variety to each complete simplicial simplicial fan $([\mathbf{F u l 9 3}])$. One can give a quotient construction of this variety using the Gale dual $\mathfrak{A}$-data as follows. Let the coordinates on $\mathfrak{g}_{\mathbb{C}}=\mathbb{C}^{n}$ be $\mathbf{x}=\left(x_{1}, \ldots, x_{n}\right)$, and define a diagonal action of the complexified torus $T_{\mathfrak{a}}=\mathfrak{a}_{\mathbb{C}} / \mathfrak{a}_{\mathbb{Z}}$ on $\mathbb{C}^{n}$ by the formula

$$
s \in \mathfrak{a}_{\mathbb{C}}, \mathbf{x} \in \mathbb{C}^{n} \mapsto\left(\exp \left(2 \pi \sqrt{-1}\left\langle\alpha_{i}, s\right\rangle\right) x_{i}, i=1, \ldots, n\right) .
$$

Given a chamber $\mathfrak{c} \in \mathfrak{C}(\mathfrak{A})$, define the open subset

$$
U_{\mathfrak{c}}=\left\{\mathrm{x} \in \mathbb{C}^{n} ; \prod_{i \in \sigma} x_{i} \neq 0 \text { for some } \sigma \text { for which } \mathfrak{c} \subset \sum_{i \in \sigma} \mathbb{R}^{>0} \alpha_{i}\right\},
$$

of $\mathbb{C}^{n}$. Then $U_{\mathfrak{c}}$ is clearly $T_{\mathfrak{a}}$-invariant, and we can define the $d$-dimensional compact variety

$$
V_{\mathfrak{c}}=U_{\mathfrak{c}} / T_{\mathfrak{a}} .
$$

As an exercise, one may find the two fans corresponding to the two chambers of our example $\mathfrak{A}_{0}$, and one may check that the corresponding two surfaces are $\mathbb{P}^{2}$ and the blow-up of $\mathbb{P}^{2}$ at a point.

Remark 1.2. Another data often used to describe toric varieties are polytopes. In our setup they appear as follows. Let $\theta \in \mathfrak{c} \cap \mathfrak{a}_{\mathbb{Z}}$ and define the partition polytope

$$
\Pi_{\theta}=\left\{\left(\gamma_{1}, \ldots, \gamma_{n}\right) \in\left(\mathbb{R}^{\geqslant 0}\right)^{n} ; \sum_{i=1}^{n} \gamma_{i} \alpha_{i}=\theta\right\} .
$$

This polytope is polar to the corresponding fan in $\mathfrak{t}$. 
Now we introduce the vector $\kappa=\sum_{i=1}^{n} \alpha_{i} \in \mathfrak{a}_{\mathbb{Z}}$. In the simplest case, which we will consider here, $\kappa$ will be in one of the chambers of $\mathfrak{A}$. The fan corresponding to this special chamber is the one induced by the faces of the convex hull of $\mathfrak{B}$. The general case is more complicated [BM02, SV04].

\section{Intersection numbers of toric varieties}

One can go one step further with the quotient construction of the previous section: given $\theta \in \mathfrak{a}_{\mathbb{Z}}^{*}$ one can define the (orbi)-line-bundle $L_{\theta}$ over $V_{\mathfrak{c}}$ as

$$
L_{\theta}=U_{\mathfrak{c}} \times_{T_{\mathfrak{a}}} \mathbb{C}_{\theta},
$$

where $\mathbb{C}_{\theta}$ is the character of $T_{\mathfrak{a}}$ corresponding to $\theta$. Taking the Chern class of this bundle, we obtain a map

$$
\chi: \mathfrak{a}_{\mathbb{Z}}^{*} \rightarrow H^{2}\left(V_{\mathfrak{c}}, \mathbb{Q}\right),
$$

which may be extended multiplicatively to a map

$$
\chi: \mathbb{C}[\mathfrak{a}] \rightarrow H^{*}\left(V_{\mathfrak{c}}, \mathbb{R}\right),
$$

i.e. to polynomials on $\mathfrak{a}$.

We are interested in the intersection numbers of the classes $\chi(\theta), \theta \in \mathfrak{a}_{\mathbb{Z}}^{*}$; this means that we are looking for a formula for the map

$$
Q \mapsto \int_{V_{\mathrm{c}}} \chi(Q)
$$

for homogeneous degree $d$ polynomials $Q$ on $\mathfrak{a}$.

First we write this intersection number as

$$
\int_{V_{\mathfrak{A}}(\mathfrak{c})} \chi(Q)=\mathrm{JK}_{\mathfrak{c}}\left(\frac{Q}{\prod_{i=1}^{n} \alpha_{i}}\right),
$$

where $\mathrm{JK}_{\mathfrak{c}}$ is a functional on the space $\mathbb{C}_{\mathfrak{A}}[\mathfrak{a}]$ of rational functions which are regular on the complement of the hyperplane arrangement

$$
U(\mathfrak{A})=\left\{u \in \mathfrak{a}_{\mathbb{C}} ; \alpha_{i}(u) \neq 0, i \in[1, n]\right\} .
$$

This functional is defined implicitly as follows: for any $r$-element subset $\sigma \subset$ $[1, n]$ we set

$$
\mathrm{JK}_{\mathfrak{c}}\left(\prod_{i \in \sigma} \frac{1}{\alpha_{i}}\right)= \begin{cases}\operatorname{vol}_{\mathfrak{a}}(\sigma)^{-1}, & \text { if } \mathfrak{c} \subset \sum_{i \in \sigma} \mathbb{R}^{>0} \alpha_{i}, \\ 0, & \text { if } \mathfrak{c} \cap \sum_{i \in \sigma} \mathbb{R}^{>0} \alpha_{i}=\emptyset,\end{cases}
$$

where $\operatorname{vol}_{\mathfrak{a}}(\sigma)$ is the volume of the parallelepiped $\sum_{i \in \sigma}[0,1] \alpha_{i}$ as measured by the lattice $\alpha_{\mathbb{Z}}$. We also declare that $\mathrm{JK}_{\mathfrak{c}}$ vanishes on fuctions of degree different 
from $-r$; using the multidimensional partial fraction expansion, it is easy to show that this defines a unique functional $\mathrm{JK}_{\mathfrak{c}}$ on $\mathbb{C}_{\mathfrak{A}}[\mathfrak{a}]$.

Let us turn to our example. Consider the chamber $\mathfrak{c}(\kappa)$ containing $\kappa=$ $2 x+3 y$. Then we obtain

$$
\int_{V_{\mathfrak{c}}} \chi\left(x^{2}\right)=\operatorname{JK}_{\mathfrak{c}(\kappa)}\left(\frac{x^{2}}{x(x+y) y^{2}}\right)=\operatorname{JK}_{\mathfrak{c}(\kappa)}\left(\frac{1}{y^{2}}-\frac{1}{(x+y) y}\right)=0-1=-1 .
$$

Similarly, we find that $\int_{V_{\mathrm{c}}} \chi\left(y^{2}\right)=0$ and $\int_{V_{\mathrm{c}}} \chi(x y)=1$.

This functional distinctly has the flavor of a residue. From this point of view, one would like to find a cycle $Z[\mathfrak{c}]$ for every $\mathfrak{c} \in \mathbb{C}(\mathfrak{A})$, which has the property that

$$
\mathrm{JK}_{\mathfrak{c}}(f)=\int_{Z[\mathfrak{c}]} f d^{r} \varphi
$$

where $d^{r} \varphi$ is the translation invariant holomorphic $r$ form normalized using the lattice $\mathfrak{a}_{\mathbb{Z}}$ and an orientation of $\mathfrak{a}$. In our example, we can let $d^{2} \varphi=d x \wedge d y$. Our main result is an explicit description of such a cycle as a solution set of $r$ polynomial equations. A priori, it is not at all clear that such a presentation exists, but here it is.

Theorem 2.1. Let $\mathfrak{c} \in \mathbb{C}(\mathfrak{A})$ be such that $\kappa \in \overline{\mathfrak{c}}$. Then for a sufficiently generic $\xi \in \mathfrak{c}$ and small $\varepsilon>0$, the real algebraic subvariety of $U(\mathfrak{A})$

$$
Z_{\varepsilon}(\xi)=\left\{u \in U(\mathfrak{A}) ; \prod_{i=1}^{n}\left|\alpha_{i}(u)\right|^{\left\langle\alpha_{i}, \lambda\right\rangle}=\varepsilon^{\langle\xi, \lambda\rangle} \text { for every } \lambda \in \mathfrak{a}_{\mathbb{Z}}\right\},
$$

when appropriately oriented, is a compact cycle which satisfies (2.3).

\section{Remark 2.2.}

1. Formally, it looks like we imposed infinitely many conditions on $Z_{\varepsilon}(\xi)$, but, in fact, because of the multiplicativity of both sides of the equations, one can reduce the number of equations to $r$. This results in an $r$-dimensional real cycle.

2. The condition $\kappa \in \overline{\mathfrak{c}}$ is crucial. Otherwise $Z_{\varepsilon}(\xi)$ might be non-compact!

3. Nevertheless, by repeating some of the $\alpha$ s sufficiently many times we can achieve the condition $\kappa \in \overline{\mathfrak{c}}$, thus we can write down a cycle for any of the chambers this way.

Returning to our example, pick $\xi=x+2 y \in \mathfrak{c}(\kappa)$ choose a basis $\left(\lambda_{1}, \lambda_{2}\right)$ dual to $(x, y)$. Our equations then have the following form

$$
|x(x+y)|=\varepsilon, \quad\left|y^{2}(x+y)\right|=\varepsilon^{2} .
$$


Naturally, the cycle for the other chamber may be realized by the system of equations where $x$ and $y$ are exchanged.

The proof of this theorem is somewhat technical, but the central idea is that of the "tropical Ansatz":

$$
|x|=\varepsilon^{a},|y|=\varepsilon^{b},|x+y|=\varepsilon^{c} .
$$

Then the equations (2.4) imply the equalities

$$
\left\{\begin{array}{l}
a+c=1, \\
2 b+c=2 .
\end{array}\right.
$$

These, naturally, do not determine $a, b$ and $c$, but we observe that for small enough $\varepsilon$, if $x+y$ is very small compared to $x$, then $x$ and $y$ should be rather close. This argument gives us the following three possibilities:

1. $c \gg a, b$, which implies $a \sim b$

2. $b \gg a, c$, which implies $a \sim c$

3. $a \gg c, b$, which implies $c \sim b$.

Here by $\gg$ we mean significantly greater, and by $\sim$ we mean rather close. Now we can go back to our system (2.5), and solve it under the three possible scenarios $a=b, a=c$, or $b=c$. We obtain the following three solutions

$$
(1,1,0), \quad(1 / 2,3 / 4,1 / 2), \quad(1 / 3,2 / 3,2 / 3) .
$$

However, only the second of these equations satisfies the corresponding inequalities! Thus we, informally, conclude, that for $\xi=x+2 y$ the cycle $Z_{\varepsilon}(\xi)$ consists of a torus which is very close to the torus $\left\{|y|=\varepsilon^{3 / 4},|x|=\varepsilon^{1 / 2}\right\} \subset \mathbb{C}^{2}$. Integration over such a torus is equivalent to an algebraic operation called iterated residue, which means ordering an $r$-tuple of $\alpha$ s and then taking the usual onedimensional residue with respect to each variable, one by one, while assuming all subsequent (from right to left) variables to be nonzero constants. Thus one check of our result is the equality

$$
\operatorname{JK}_{\mathfrak{c}(\kappa)}(f)=\operatorname{Res}_{x} \operatorname{Res}_{y} f d x d y
$$

which is easy to see.

All is not as simple as it appears, however. Take another $\xi$, say, let $\xi=$ $3 x+4 y$. Completing the above computation, we obtain two solutions: $(1,1,2)$ and $(5 / 3,4 / 3,4 / 3)$ instead of one!!!

It looks like there is an error but no: the union of the two tori we obtain this way is, in fact, homologous in $\left\{(x, y) \in \mathbb{C}^{2} ; x, y, x+y \neq 0\right\}$ to the single torus we computed above. 
If you are intrigued, check out $[\mathbf{S V 0 4}, \mathbf{S V}]$ for details and proofs! In [BM02, BM03] you can learn what all this has to do with mirror symmetry, and [Stu02] will explain why this method is called tropical.

\section{References}

[BM02] V. V. BATYReV \& E. N. MATERov - Toric residues and mirror symmetry, Mosc. Math. J. 2 (2002), no. 3, p. 435-475, Dedicated to Yuri I. Manin on the occasion of his 65 th birthday.

[BM03] _ Mixed toric residues and Calabi-Yau complete intersections, in Calabi-Yau varieties and mirror symmetry (Toronto, ON, 2001), Fields Inst. Commun., vol. 38, Amer. Math. Soc., Providence, RI, 2003, p. 3-26.

[Ful93] W. Fulton - Introduction to toric varieties, Annals of Mathematics Studies, vol. 131, Princeton University Press, Princeton, NJ, 1993, The William H. Roever Lectures in Geometry.

[Stu02] B. StuRmfels - Solving systems of polynomial equations, CBMS Regional Conference Series in Mathematics, vol. 97, Published for the Conference Board of the Mathematical Sciences, Washington, DC, 2002.

[SV] A. Szenes \& M. VERGne - Mixed Toric Residues and tropical degenerations, math. AG/0410064.

[SV04] A. Szenes \& M. VERGNe - Toric reduction and a conjecture of Batyrev and Materov, Invent. Math. 158 (2004), no. 3, p. 453-495. 

Mathematisches Institut, Seminars, (Y. TschINKel, ed.), p. 117-137

Universität Göttingen, 2004-05

\title{
A SPECTRAL INTERPRETATION FOR THE ZEROS OF THE RIEMANN ZETA FUNCTION
}

\author{
R. Meyer \\ Mathematisches Institut, Westfälische Wilhelms-Universität Münster, \\ Einsteinstrasse 62, 48149 Münster, Germany \\ E-mail : rameyer@math.uni-muenster.de
}

\begin{abstract}
Based on work of Alain Connes, I have constructed a spectral interpretation for zeros of $L$-functions. Here we specialise this construction to the Riemann $\zeta$-function. We construct an operator on a nuclear Fréchet space whose spectrum is the set of non-trivial zeros of $\zeta$. We exhibit the explicit formula for the zeros of the Riemann $\zeta$-function as a character formula.
\end{abstract}

\section{Introduction}

The purpose of this note is to explain what the spectral interpretation for zeros of $L$-functions in [Mey] amounts to in the simple special case of the Riemann $\zeta$-function. The article [Mey] is inspired by the work of Alain Connes in $\left[\right.$ Con99]. We will construct a nuclear Fréchet space $\mathscr{H}_{-}^{0}$ and an operator $D_{-}$ on $\mathscr{H}_{-}^{0}$ whose spectrum is equal to the set of non-trivial zeros of the Riemann $\zeta$-function $\zeta(s)=\sum_{n=1}^{\infty} n^{-s}$. By definition, the non-trivial zeros of $\zeta$ are the zeros of the complete $\zeta$-function

$$
\xi(s)=\pi^{-s / 2} \Gamma(s / 2) \zeta(s) .
$$

In addition, the algebraic multiplicity of $s$ as an eigenvalue of $D_{-}$is the zero order of $\xi$ at $s$. Thus $D_{-}$is a spectral interpretation for the zeros of $\xi$.

November 11, 2004.

This research was supported by the EU-Network Quantum Spaces and Noncommutative Geometry (Contract HPRN-CT-2002-00280) and the Deutsche Forschungsgemeinschaft (SFB 478). 
We construct $D_{-}$as the generator of a smooth representation $\rho_{-}$of $\mathbb{R}_{+}^{\times} \cong \mathbb{R}$ on $\mathscr{H}_{-}^{0}$. Although the single operator $D_{-}$is more concrete, it is usually better to argue with the representation $\rho_{-}$instead. Let $\mathscr{D}\left(\mathbb{R}_{+}^{\times}\right)$be the convolution algebra of smooth, compactly supported functions on $\mathbb{R}_{+}^{\times}$. The integrated form of $\rho_{-}$is a bounded algebra homomorphism $\int \rho_{-}: \mathscr{D}\left(\mathbb{R}_{+}^{\times}\right) \rightarrow \operatorname{End}\left(\mathscr{H}_{-}^{0}\right)$. We show that $\rho_{-}$is a summable representation in the notation of $[\mathbf{M e y}]$. That is, $\int \rho_{-}(f)$ is a nuclear operator for all $f \in \mathscr{D}\left(\mathbb{R}_{+}^{\times}\right)$. The character $\chi\left(\rho_{-}\right)$is the distribution on $\mathbb{R}_{+}^{\times}$defined by $\chi\left(\rho_{-}\right)(f)=\operatorname{tr} \int \rho_{-}(f)$. The representation $\rho_{-}$is part of a virtual representation $\rho=\rho_{+} \ominus \rho_{-}$, where $\rho_{+}$is a spectral interpretation for the poles of $\xi$. That is, $\rho_{+}$is 2 -dimensional and its generator $D_{+}$has eigenvalues 0 and 1 . We interpret $\rho$ as a formal difference of $\rho_{+}$and $\rho_{-}$and therefore define $\chi(\rho):=\chi\left(\rho_{+}\right)-\chi\left(\rho_{-}\right)$.

The spectrum of $\rho$ consists exactly of the poles and zeros of $\xi$, and the spectral multiplicity (with appropriate signs) of $s \in \mathbb{C}$ is the order of $\xi$ at $s$, which is positive at the two poles 0 and 1 and negative at the zeros of $\xi$. The spectral computation of the character yields

$$
\chi(\rho)(f)=\sum_{s \in \mathbb{C}} \operatorname{ord}_{\xi}(s) \hat{f}(s), \quad \text { where } \quad \hat{f}(s):=\int_{0}^{\infty} f(x) x^{s} \frac{d x}{x} .
$$

If an operator has a sufficiently nice integral kernel, then we can also compute its trace by integrating its kernel along the diagonal. This recipe applies to $\int \rho_{-}(f)$ and yields another formula for $\chi\left(\rho_{-}\right)$. Namely,

$$
\chi(\rho)=W=\sum_{p \in \mathscr{P}} W_{p}+W_{\infty},
$$

where $\mathscr{P}$ is the set of primes,

$$
\begin{aligned}
W_{p}(f) & =\sum_{e=1}^{\infty} f\left(p^{-e}\right) p^{-e} \ln (p)+\sum_{e=1}^{\infty} f\left(p^{e}\right) \ln (p), \\
W_{\infty}(f) & =p v \int_{0}^{\infty} \frac{f(x)}{|1-x|}+\frac{f(x)}{1+x} d x .
\end{aligned}
$$

The distribution $W_{\infty}$ involves a principal value because the integrand may have a pole at 1. Equating the two formulas for $\chi(\rho)$, we get the well-known explicit formula that relates zeros of $\xi$ and prime numbers.

We do not need the functions $\zeta$ and $\xi$ to define our spectral interpretation. Instead we use an operator $Z$, called the Zeta operator, which is closely related to the $\zeta$-function. This operator is the key ingredient in our construction. In addition, we have to choose the domain and target space of $Z$ rather carefully. 
It is possible to prove the Prime Number Theorem using the representation $\rho$ instead of the $\zeta$-function. The only input that we need is that the distribution $W$ is quantised, that is, of the form $\sum n(s) \hat{f}(s)$ with some function $n: \mathbb{C} \rightarrow \mathbb{Z}$ (see [Mey]).

Our constructions for the $\zeta$-function can be generalised to Dirichlet $L$-functions. We indicate how this is done in the last section. We run into problems, however, for number fields with more than one infinite place. There are also other conceptual and aesthetic reasons for prefering adelic constructions as in [Mey]. Our goal here is only to make these constructions more explicit in a simple special case.

\section{The ingredients: some function spaces and operators}

Let $\mathscr{S}(\mathbb{R})$ be the Schwartz space of $\mathbb{R}$. Thus $f: \mathbb{R} \rightarrow \mathbb{C}$ belongs to $\mathscr{S}(\mathbb{R})$ if and only if all its derivatives $f^{(n)}$ are rapidly decreasing in the sense that $f^{(n)}(x)=O\left(|x|^{-s}\right)$ for $|x| \rightarrow \infty$ for all $s \in \mathbb{R}_{+}, n \in \mathbb{N}$. We topologise $\mathscr{S}(\mathbb{R})$ in the usual fashion. The convolution turns $\mathscr{S}(\mathbb{R})$ into a Fréchet algebra.

We remark that we neither gain nor loose anything if we view $\mathscr{S}(\mathbb{R})$ as a bornological vector space as in [Mey]. All function spaces that we shall need are Fréchet spaces, so that bornological and topological analysis are equivalent. The bornological point of view only becomes superior if we mix $\mathscr{S}(\mathbb{R})$ with spaces like $\mathscr{S}\left(\mathbb{Q}_{p}\right)$, which are not Fréchet.

We use the natural logarithm $\ln$ to identify the multiplicative group $\mathbb{R}_{+}^{\times}$ with $\mathbb{R}$. This induces an isomorphism between the Schwartz algebras $\mathscr{S}\left(\mathbb{R}_{+}^{\times}\right)$ and $\mathscr{S}(\mathbb{R})$. The standard Lebesgue measure on $\mathbb{R}$ corresponds to the Haar measure $d^{\times} x=x^{-1} d x$ on $\mathbb{R}_{+}^{\times}$. We always use this measure in the following.

We let

$$
\mathscr{S}\left(\mathbb{R}_{+}^{\times}\right)_{s}=\mathscr{S}\left(\mathbb{R}_{+}^{\times}\right) \cdot x^{-s}=\left\{f: \mathbb{R}_{+}^{\times} \rightarrow \mathbb{C} \mid\left(x \mapsto f(x) x^{s}\right) \in \mathscr{S}\left(\mathbb{R}_{+}^{\times}\right)\right\}
$$

for $s \in \mathbb{R}$ and

$$
\mathscr{S}\left(\mathbb{R}_{+}^{\times}\right)_{I}=\bigcap_{s \in I} \mathscr{S}\left(\mathbb{R}_{+}^{\times}\right)_{s}
$$

for an interval $I \subseteq \mathbb{R}$. We will frequently use that

$$
\mathscr{S}\left(\mathbb{R}_{+}^{\times}\right)_{[a, b]}=\mathscr{S}\left(\mathbb{R}_{+}^{\times}\right)_{a} \cap \mathscr{S}\left(\mathbb{R}_{+}^{\times}\right)_{b} .
$$

The reason for this is that $\mathscr{S}\left(\mathbb{R}_{+}^{\times}\right)$is closed under multiplication by $\left(x^{\varepsilon}+x^{-\delta}\right)^{-1}$ for $\varepsilon, \delta \geq 0$.

Hence $\mathscr{S}\left(\mathbb{R}_{+}^{\times}\right)_{[a, b]}$ becomes a Fréchet space in a canonical way. Exhausting $I$ by an increasing sequence of compact intervals, we may turn $\mathscr{S}\left(\mathbb{R}_{+}^{\times}\right)_{I}$ into a Fréchet space for general $I$. Since $x \mapsto x^{s}$ is a character of $\mathbb{R}_{+}^{\times}$, the spaces 
$\mathscr{S}\left(\mathbb{R}_{+}^{\times}\right)_{s}$ for $s \in \mathbb{R}$ are closed under convolution. Hence $\mathscr{S}\left(\mathbb{R}_{+}^{\times}\right)_{I}$ is closed under convolution as well and becomes a Fréchet algebra. We are particularly interested in

$$
\begin{aligned}
\mathscr{H}_{-}=\mathscr{O}\left(\mathbb{R}_{+}^{\times}\right) & :=\mathscr{S}\left(\mathbb{R}_{+}^{\times}\right)_{-\infty, \infty[}, \\
\mathscr{S}_{>} & :=\mathscr{S}\left(\mathbb{R}_{+}^{\times}\right)_{1, \infty[}, \\
\mathscr{S}_{<} & :=\mathscr{S}\left(\mathbb{R}_{+}^{\times}\right)_{-\infty, 0[} .
\end{aligned}
$$

We also let

$$
\mathscr{H}_{+}:=\{f \in \mathscr{S}(\mathbb{R}) \mid f(x)=f(-x) \text { for all } x \in \mathbb{R}\} .
$$

The spaces $\mathscr{H}_{ \pm}$will be crucial for our spectral interpretation; the spaces $\mathscr{S}_{>}$ and $\mathscr{S}_{<}$only play an auxiliary role as sufficiently large spaces in which the others can be embedded.

Given topological vector spaces $A$ and $B$, we write $A \prec B$ to denote that $A$ is contained in $B$ and that the inclusion is a continuous linear map. Clearly,

$$
\mathscr{S}\left(\mathbb{R}_{+}^{\times}\right)_{I} \prec \mathscr{S}\left(\mathbb{R}_{+}^{\times}\right)_{J} \quad \text { if } I \supseteq J .
$$

The group $\mathbb{R}_{+}^{\times}$acts on $\mathscr{S}\left(\mathbb{R}_{+}^{\times}\right)_{I}$ and $\mathscr{H}_{+}$by the regular representation

$$
\lambda_{t} f(x):=f\left(t^{-1} x\right) \quad \text { for } t, x \in \mathbb{R}_{+}^{\times} .
$$

Its integrated form is given by the same formula as the convolution:

$$
\int \lambda(h) f(x):=\int_{0}^{\infty} h(t) f\left(t^{-1} x\right) d^{\times} t .
$$

We denote the projective complete topological tensor product by $\hat{\otimes}$ (see [Gro55]). If $V$ and $W$ are Fréchet spaces, so is $V \hat{\otimes} W$. We want to know $\mathscr{S}\left(\mathbb{R}_{+}^{\times}\right)_{I} \hat{\otimes} \mathscr{S}\left(\mathbb{R}_{+}^{\times}\right)_{J}$ for two intervals $I, J$. Since both tensor factors are nuclear Fréchet spaces, this is easy enough to compute. We find

$$
\begin{aligned}
& \mathscr{S}\left(\mathbb{R}_{+}^{\times}\right)_{I} \hat{\otimes} \mathscr{S}\left(\mathbb{R}_{+}^{\times}\right)_{J} \\
& \quad \cong\left\{f:\left(\mathbb{R}_{+}^{\times}\right)^{2} \rightarrow \mathbb{C} \mid f(x, y) \cdot x^{s} y^{t} \in \mathscr{S}\left(\left(\mathbb{R}_{+}^{\times}\right)^{2}\right) \text { for all } s \in I, t \in J\right\}
\end{aligned}
$$

with the canonical topology. This follows easily from $\mathscr{S}(\mathbb{R}) \hat{\otimes} \mathscr{S}(\mathbb{R}) \cong \mathscr{S}\left(\mathbb{R}^{2}\right)$ and the compatibility of $\hat{\otimes}$ with inverse limits.

We shall need the Fourier transform

$$
\mathfrak{F}: \mathscr{S}(\mathbb{R}) \rightarrow \mathscr{S}(\mathbb{R}), \quad \mathfrak{F} f(y):=\int_{\mathbb{R}} f(y) \exp (2 \pi \mathrm{i} x y) d x .
$$

Notice that $\mathfrak{F} f$ is even if $f$ is. Hence $\mathfrak{F}$ restricts to an operator on $\mathscr{H}_{+} \subseteq \mathscr{S}(\mathbb{R})$. In the following, we usually restrict $\mathfrak{F}$ to this subspace. It is well-known that 
$\mathfrak{F}^{-1} f(y)=\mathfrak{F} f(-y)$ for all $f \in \mathscr{S}(\mathbb{R}), y \in \mathbb{R}$. Hence

$$
\mathfrak{F}^{2}=\text { id } \quad \text { as operators on } \mathscr{H}_{+} \text {. }
$$

Since $\mathfrak{F}$ is unitary on $L^{2}(\mathbb{R}, d x)$, it is also unitary on the subspace of even functions, which is isomorphic to $L^{2}\left(\mathbb{R}_{+}^{\times}, x d^{\times} x\right)$.

We also need the involution

$$
J: \mathscr{O}\left(\mathbb{R}_{+}^{\times}\right) \rightarrow \mathscr{O}\left(\mathbb{R}_{+}^{\times}\right), \quad J f(x):=x^{-1} f\left(x^{-1}\right) .
$$

We have $J^{2}=$ id. One checks easily that $J$ extends to a unitary operator on $L^{2}\left(\mathbb{R}_{+}^{\times}, x d^{\times} x\right)$ and to an isomorphism of topological vector spaces

$$
J: \mathscr{S}\left(\mathbb{R}_{+}^{\times}\right)_{I} \cong \mathscr{S}\left(\mathbb{R}_{+}^{\times}\right)_{1-I}
$$

for any interval $I$. Especially, $J$ is an isomorphism between $\mathscr{S}_{<}$and $\mathscr{S}_{>}$.

We have

$$
\int \lambda(h) \circ \mathfrak{F}=\mathfrak{F} \circ \int \lambda(J h), \quad \int \lambda(h) \circ J=J \circ \int \lambda(J h)
$$

for all $h \in \mathscr{D}\left(\mathbb{R}_{+}^{\times}\right)$. Hence the composites $\mathfrak{F} J$ and $J \mathfrak{F}=(\mathfrak{F} J)^{-1}$, which are unitary operators on $L^{2}\left(\mathbb{R}_{+}^{\times}, x d^{\times} x\right)$, commute with the regular representation $\lambda$.

A multiplier of $\mathscr{S}\left(\mathbb{R}_{+}^{\times}\right)_{I}$ is a continuous linear operator on $\mathscr{S}\left(\mathbb{R}_{+}^{\times}\right)_{I}$ that commutes with the regular representation.

Proposition 2.1. Viewing $\mathscr{H}_{+} \subseteq L^{2}\left(\mathbb{R}_{+}^{\times}, x d^{\times} x\right)$, we have

$$
\begin{aligned}
& \mathscr{H}_{+}=\left\{f \in L^{2}\left(\mathbb{R}_{+}^{\times}, x d^{\times} x\right) \mid f \in \mathscr{S}\left(\mathbb{R}_{+}^{\times}\right)_{0, \infty} \text { and } J \mathfrak{F}(f) \in \mathscr{S}\left(\mathbb{R}_{+}^{\times}\right)_{-\infty, 1}\right\} . \\
& \text { - The operator } \left.\mathfrak{F} J \text { is a multiplier of } \mathscr{S}\left(\mathbb{R}_{+}^{\times}\right)_{I} \text { for } I \subseteq\right] 0, \infty[\text {. } \\
& \text { - The operator } \left.J \mathfrak{F} \text { is a multiplier of } \mathscr{S}\left(\mathbb{R}_{+}^{\times}\right)_{I} \text { for } I \subseteq\right]-\infty, 1[. \\
& \text { - Hence } \left.\mathfrak{F} J \text { and } J \mathfrak{F} \text { are invertible multipliers of } \mathscr{S}\left(\mathbb{R}_{+}^{\times}\right)_{I} \text { for } I \subseteq\right] 0,1[\text {. }
\end{aligned}
$$

Proof. We first have to describe $\mathscr{S}\left(\mathbb{R}_{+}^{\times}\right)_{I}$ more explicitly. For simplicity, we assume the interval $I$ to be open. Let $D f(x):=x \cdot f^{\prime}(x)$. This differential operator is the generator of the representation $\lambda$ of $\mathbb{R}_{+}^{\times} \cong \mathbb{R}$. Let $f \in L^{2}\left(\mathbb{R}_{+}^{\times}, x d^{\times} x\right)$. Then $f \in \mathscr{S}\left(\mathbb{R}_{+}^{\times}\right)_{I}$ if and only if $D^{m}\left(f \cdot x^{s}\right) \cdot(\ln x)^{k} \in L^{2}\left(\mathbb{R}_{+}^{\times}, d^{\times} x\right)$ for all $m, k \in \mathbb{N}, s \in I$. Using the Leibniz rule, one shows that this is equivalent to $D^{m}(f) \cdot x^{s} \cdot(\ln x)^{k} \in L^{2}\left(\mathbb{R}_{+}^{\times}, d^{\times} x\right)$ for all $m, k \in \mathbb{N}, s \in I$. Since $I$ is open, we may replace $x^{s}$ by $x^{s+\varepsilon}+x^{s-\varepsilon}$ for some $\varepsilon>0$. This dominates $x^{s}(\ln x)^{k}$ for any $k \in \mathbb{N}$, so that it suffices to require $\left(D^{m} f\right) \cdot x^{s} \in L^{2}\left(\mathbb{R}_{+}^{\times}, d^{\times} x\right)$ for all $m \in \mathbb{N}, s \in I$.

This description of $\mathscr{S}\left(\mathbb{R}_{+}^{\times}\right)_{I}$ easily implies $\mathscr{H} \mathscr{H}_{+} \subseteq \mathscr{S}\left(\mathbb{R}_{+}^{\times}\right)_{0, \infty}$. Since $\mathfrak{F}$ maps $\mathscr{H}+$ to itself and $J$ maps $\mathscr{S}\left(\mathbb{R}_{+}^{\times}\right)_{] 0, \infty[}$ to $\mathscr{S}\left(\mathbb{R}_{+}^{\times}\right)_{]-\infty, 1}$, we get " $\subseteq$ " in (10). 
Conversely, $f \in \mathscr{H}_{+}$if and only if $f$ and $\mathfrak{F} f$ are both $O\left(x^{-s}\right)$ for $|x| \rightarrow \infty$ for all $s \in \mathbb{N}$. This yields " $\supseteq$ " in (10) and finishes the proof of (10).

In the following computation, we describe $\mathscr{O}\left(\mathbb{R}_{+}^{\times}\right) \hat{\otimes} \mathscr{S}\left(\mathbb{R}_{+}^{\times}\right)_{I}$ as in (4). Choose any $\psi \in \mathscr{O}\left(\mathbb{R}_{+}^{\times}\right)$with $\int_{0}^{\infty} \psi(x) d^{\times} x=1$. Then $\sigma f(x, y):=\psi(x) f(x y)$ defines a continuous linear map from $\mathscr{S}\left(\mathbb{R}_{+}^{\times}\right)_{I}$ to $\mathscr{O}\left(\mathbb{R}_{+}^{\times}\right) \hat{\otimes} \mathscr{S}\left(\mathbb{R}_{+}^{\times}\right)_{I}$ by (4). This is a section for the convolution map

$$
\int \lambda: \mathscr{O}\left(\mathbb{R}_{+}^{\times}\right) \hat{\otimes} \mathscr{S}\left(\mathbb{R}_{+}^{\times}\right)_{I} \rightarrow \mathscr{S}\left(\mathbb{R}_{+}^{\times}\right)_{I}, \quad\left(\int \lambda f\right)(x)=\int_{0}^{\infty} f\left(t, t^{-1} x\right) d^{\times} t .
$$

We have $\mathscr{O}\left(\mathbb{R}_{+}^{\times}\right)=J \mathscr{O}\left(\mathbb{R}_{+}^{\times}\right) \subset \mathscr{H}_{+}=\mathfrak{F} \mathscr{H}_{+} \subset \mathscr{S}\left(\mathbb{R}_{+}^{\times}\right)_{I}$ if $\left.I \subseteq\right] 0, \infty[$. Hence we get a continuous linear operator

$$
\mathscr{S}\left(\mathbb{R}_{+}^{\times}\right)_{I} \stackrel{\sigma}{\longrightarrow} \mathscr{O}\left(\mathbb{R}_{+}^{\times}\right) \hat{\otimes} \mathscr{S}\left(\mathbb{R}_{+}^{\times}\right)_{I} \stackrel{\mathfrak{F} J \hat{\otimes} \mathrm{id}}{\longrightarrow} \mathscr{S}\left(\mathbb{R}_{+}^{\times}\right)_{I} \hat{\otimes} \mathscr{S}\left(\mathbb{R}_{+}^{\times}\right)_{I} \stackrel{\rho \lambda}{\longrightarrow} \mathscr{S}\left(\mathbb{R}_{+}^{\times}\right)_{I} .
$$

The last map exists because $\mathscr{S}\left(\mathbb{R}_{+}^{\times}\right)_{I}$ is a convolution algebra. If we plug $f_{0} * f_{1}$ with $f_{0}, f_{1} \in \mathscr{O}\left(\mathbb{R}_{+}^{\times}\right)$into this operator, we get $\mathfrak{F} J\left(f_{0} * f_{1}\right)$ because $\sigma$ is a section for $\int \lambda$ and because of (9). Since products $f_{0} * f_{1}$ are dense in $\mathscr{O}\left(\mathbb{R}_{+}^{\times}\right)$, the above operator on $\mathscr{S}\left(\mathbb{R}_{+}^{\times}\right)_{I}$ extends $\mathfrak{F} J$ on $\mathscr{O}\left(\mathbb{R}_{+}^{\times}\right)$.

Now (8) implies the continuity of $J \mathfrak{F}=J(\mathfrak{F} J) J$ on $\mathscr{S}\left(\mathbb{R}_{+}^{\times}\right)_{I}$ for $\left.I \subseteq\right]-\infty, 1[$. Hence both $J \mathfrak{F}$ and $\mathfrak{F} J$ are multipliers of $\mathscr{S}\left(\mathbb{R}_{+}^{\times}\right)_{I}$ for $\left.I \subseteq\right] 0,1[$. They are inverse to each other on $\mathscr{S}\left(\mathbb{R}_{+}^{\times}\right)_{I}$ because they are inverse to each other on $L^{2}\left(\mathbb{R}_{+}^{\times}, x d^{\times} x\right)$.

\section{The Zeta operator and the Poisson Summation Formula}

In this section we study the properties of the following operator:

Definition 3.1. The Zeta operator is defined by

$$
Z f(x):=\sum_{n=1}^{\infty} f(n x)=\sum_{n=1}^{\infty} \lambda_{n}^{-1} f(x)
$$

for $f \in \mathscr{H}_{+}, x \in \mathbb{R}_{+}^{\times}$.

Let $\check{\zeta}$ be the distribution $\sum_{n=1}^{\infty} \delta_{n}^{-1}: \psi \mapsto \sum_{n=1}^{\infty} \psi\left(n^{-1}\right)$. Then

$$
\check{\zeta}(s)=\sum_{n=1}^{\infty} n^{-s}=\zeta(s), \quad Z f=\int \lambda(\check{\zeta})(f) .
$$

Thus the data $Z, \zeta$, and $\check{\zeta}$ are equivalent. 
The Euler product expansion of the $\zeta$-function takes the following form in this picture. Let $\mathscr{P}$ be the set of prime numbers in $\mathbb{N}^{*}$. We have

$$
Z=\prod_{p \in \mathscr{P}} \sum_{e=0}^{\infty} \lambda_{p^{-e}}=\prod_{p \in \mathscr{P}}\left(1-\lambda_{p}^{-1}\right)^{-1}
$$

Hence we get a candidate for an inverse of $Z$ :

$$
Z^{-1} f(x)=\prod_{p \in \mathscr{P}}\left(1-\lambda_{p}^{-1}\right) f(x)=\sum_{n=1}^{\infty} \mu(n) f(n x) .
$$

Here $\mu(n)$ is the usual Möbius function; it vanishes unless $n$ is square-free, and is $(-1)^{j}$ if $n$ is a product of $j$ different prime numbers.

The following assertion is equivalent to the absolute convergence in the region $\operatorname{Re} s>1$ of the Euler product defining $\zeta(s)$.

Proposition 3.2. $Z$ and $Z^{-1}$ are continuous linear operators on $\mathscr{S}_{>}$that are inverse to each other.

Proof. Let $D f(x)=x f^{\prime}(x)$. We have observed above that $f \in \mathscr{S}_{>}$if and only if $D^{m} f \in L^{2}\left(\mathbb{R}_{+}^{\times}, x^{2 s} d^{\times} x\right)$ for all $m \in \mathbb{N}, s>1$. We check that $Z$ and $Z^{-1}$ preserve this estimate. The operator $\lambda_{t}$ for $t \in \mathbb{R}_{+}^{\times}$has norm $\left\|\lambda_{t}\right\|^{s}=t^{s}$ on $L^{2}\left(\mathbb{R}_{+}^{\times}, x^{2 s} d^{\times} x\right)$ for all $s \in \mathbb{R}$. Hence the same estimates that yield the absolute convergence of the Euler product for $\zeta(s)$ also show that the products $\prod_{p \in \mathscr{P}}\left(1-\lambda_{p}^{-1}\right)^{ \pm 1}$ converge absolutely with respect to the operator norm on $L^{2}\left(\mathbb{R}_{+}^{\times}, x^{2 s} d^{\times} x\right)$ for $s>1$. Since all factors commute with $D$, this remains true if we replace $L^{2}\left(\mathbb{R}_{+}^{\times}, x^{2 s} d^{\times} x\right)$ by the Sobolev space defined by the norm

$$
\|f\|_{2}^{m, s}:=\int_{0}^{\infty}\left|D^{m} f(x)\right|^{2} x^{2 s} d^{\times} x .
$$

Thus $Z$ and $Z^{-1}$ are continuous linear operators on these Sobolev spaces for all $m \in \mathbb{N}$ and all $s>1$. This yields the assertion.

Next we recall the Poisson Summation Formula. It asserts that

$$
\sum_{n \in \mathbb{Z}} f(x n)=x^{-1} \sum_{n \in \mathbb{Z}} \mathfrak{F} f\left(x^{-1} n\right)
$$

for all $x \in \mathbb{R}_{+}^{\times}$and all $f \in \mathscr{S}(\mathbb{R})$. For $f \in \mathscr{H}_{+}$, this becomes

$$
f(0) / 2+Z f(x)=J Z \mathfrak{F} f(x)+x^{-1} \cdot \mathfrak{F} f(0) / 2 .
$$

Let

$$
\mathscr{H}_{\cap}:=\{f \in \mathscr{S}(\mathbb{R}) \mid f(0)=\mathfrak{F} f(0)=0\} .
$$


This is a closed, $\lambda$-invariant subspace of $\mathscr{S}(\mathbb{R})$. If $f \in \mathscr{H}_{\cap}$, then (11) simplifies to $Z f=J Z \mathfrak{F} f$.

Let $h_{0}: \mathbb{R}_{+}^{\times} \rightarrow[0,1]$ be a smooth function with $h_{0}(t)=1$ for $t \ll 1$ and $h_{0}(t)=0$ for $t \gg 1$. Let $\mathscr{H}_{\cup}$ be the space of functions on $\mathbb{R}_{+}^{\times}$that is generated by $\mathscr{H}_{-}=\mathscr{O}\left(\mathbb{R}_{+}^{\times}\right)$and the two additional functions $h_{0}$ and $x^{-1} \cdot h_{0}$. The regular representation extends to $\mathscr{H}_{\cup}$. Writing $\mathbb{C}\left(x^{s}\right)$ for $\mathbb{C}$ equipped with the representation by the character $x^{s}$, we get an extension of representations

$$
\mathscr{H}_{-} \longmapsto \mathscr{H}_{\cup} \rightarrow \mathbb{C}\left(x^{0}\right) \oplus \mathbb{C}\left(x^{1}\right) .
$$

Theorem 3.3. The Zeta operator is a continuous linear map $Z: \mathscr{H}_{+} \rightarrow$ $\mathscr{H}_{\cup}$. Even more, this map has closed range and is a topological isomorphism onto its range. We have $Z f \in \mathscr{H}_{-}$if and only if $f \in \mathscr{H}_{\cap}$.

Proof. Proposition 2.1 yields $\mathscr{H}_{+} \prec \mathscr{S}_{>}$. By Proposition 3.2, $Z$ is continuous on $\mathscr{S}_{>}$. Hence we get continuity of $Z: \mathscr{H}_{+} \rightarrow \mathscr{S}_{>}$. Similarly, $J Z \mathfrak{F}$ is a continuous linear operator $\mathscr{H}_{+} \rightarrow \mathscr{S}_{<}$. By (11), $Z$ restricts to a continuous linear map

$$
\mathscr{H}_{\cap} \rightarrow \mathscr{S}_{>} \cap \mathscr{S}_{<}=\mathscr{H}_{-} .
$$

Equation (11) also implies that $Z f$ still belongs to $\mathscr{H}_{\cup}$ for arbitrary $f \in \mathscr{H}_{+}$ and that $Z f \in \mathscr{H}_{-}$if and only if $f \in \mathscr{H}_{\cap}$.

It remains to prove that $Z$ is a topological isomorphism onto its range. This implies that the range is closed because all spaces involved are complete. It suffices to prove that the restriction $Z: \mathscr{H}_{\cap} \rightarrow \mathscr{H}_{-}$is an isomorphism onto its range. Equivalently, a sequence $\left(f_{n}\right)$ in $\mathscr{H}_{n}$ converges if and only if $\left(Z f_{n}\right)$ converges in $\mathscr{H}_{-}$. One implication is contained in the continuity of $Z$. Suppose that the sequence $\left(Z f_{n}\right)$ converges in $\mathscr{H}_{-}$. Hence it converges in both $\mathscr{S}_{>}$ and $\mathscr{S}_{<}$. Equation (11) yields $Z f_{n}=J Z \mathfrak{F} f_{n}$. Using (8), we get that both $\left(Z f_{n}\right)$ and $\left(Z \mathfrak{F} f_{n}\right)$ converge in $\mathscr{S}_{>}$. Proposition 3.2 yields that $\left(f_{n}\right)$ and $\left(\mathfrak{F} f_{n}\right)$ converge in $\mathscr{S}_{>}$.

Therefore, $\left(D^{m} f_{n} \cdot x^{s}\right)$ and $\left(D^{m} \mathfrak{F} f_{n} \cdot x^{s}\right)$ converge in $L^{2}(\mathbb{R}, d x)$ for all $s>1 / 2$, where $D f(x)=x f^{\prime}(x)$. Hence $x^{k}(d / d x)^{l} f_{n}$ and $(d / d x)^{k}\left(x^{l} f_{n}\right)$ converge if $k, l \in$ $\mathbb{N}$ satisfy $k>l+1$. The first condition implies convergence in $\mathscr{S}(\mathbb{R} \backslash]-1,1[)$ because $x \geq 1$ in this region. The second condition contains convergence of $(d / d x)^{k} f_{n}$ in $L^{2}([-1,1], d x)$. Hence both conditions together imply convergence in $\mathscr{S}(\mathbb{R})$ as desired.

We now discuss the close relationship between the above theorem and the meromorphic continuation of the $\zeta$-function and the functional equation (see also [Tat67]). Recall that $\hat{f}(s):=\int_{0}^{\infty} f(x) x^{s} d^{\times} x$. This defines an entire 
function for $f \in \mathscr{H}_{-}$. We have described $Z$ as the convolution with the distribution $\check{\zeta}$ on $\mathbb{R}_{+}^{\times}$, which satisfies $\check{\zeta}(s)=\zeta(s)$. Therefore,

$$
(Z f) \uparrow(s)=\zeta(s) \hat{f}(s)
$$

for all $f \in \mathscr{S}_{>}, s \in \mathbb{C}$ with $\operatorname{Re} s>1$. The Poisson Summation Formula implies $Z f \in \mathscr{H}_{-}$for $f \in \mathscr{H}_{\cap}$, so that $\zeta(s) \hat{f}(s)$ extends to an entire function. Especially, this holds if $f \in \mathscr{H}_{-}$satisfies $\hat{f}(1)=0$. For such $f$, the function $\hat{f}(s)$ is an entire function on $\mathbb{C}$ as well. Therefore, $\zeta$ has a meromorphic continuation to all of $\mathbb{C}$. For any $s \neq 1$, there exists $f \in \mathscr{H}_{-}$with $\hat{f}(1)=0$ and $\hat{f}(s) \neq 0$. Therefore, the only possible pole of $\zeta$ is at 1 .

It is easy to see that $(J f) \mathcal{Y}(s)=\hat{f}(1-s)$. Hence (11) implies

$$
\zeta(1-s)(J \mathfrak{F} f)\}(s)=\zeta(s) \hat{f}(s)
$$

for all $s \in \mathbb{C}, f \in \mathscr{H}_{\cap}$. This equation still holds for $f \in \mathscr{H}_{+}$by $\mathbb{R}_{+}^{\times}$-equivariance. Now we plug in the special function $f(x)=2 \exp \left(-\pi x^{2}\right)$, which satisfies $\mathfrak{F} f=f$ and $\hat{f}(s)=\pi^{-s / 2} \Gamma(s / 2)$. Thus $\zeta(s) \hat{f}(s)=\xi(s)$ is the complete $\zeta$-function. Equation (14) becomes the functional equation $\xi(1-s)=\xi(s)$.

\section{The spectral interpretation}

Let $Z \mathscr{H}_{+} \subseteq \mathscr{H}_{\cup}$ be the range of $Z$. This is a closed subspace of $\mathscr{H}_{\cup}$ and topologically isomorphic to $\mathscr{H}_{+}$by Theorem 3.3. Moreover,

$$
Z \mathscr{H}_{\cap}=Z \mathscr{H}_{+} \cap \mathscr{H}_{-}, \quad \mathscr{H}_{\cup}=Z \mathscr{H}_{+}+\mathscr{H}_{-} .
$$

We define

$$
\begin{aligned}
& \mathscr{H}_{+}^{0}:=\mathscr{H}_{+} / \mathscr{H}_{\cap} \cong \mathscr{H}_{\cup} / \mathscr{H}_{-} \\
& \mathscr{H}_{-}^{0}:=\mathscr{H}_{-} / Z \mathscr{H}_{\cap} \cong \mathscr{H}_{\cup} / Z \mathscr{H}_{+} .
\end{aligned}
$$

We equip $\mathscr{H}_{ \pm}^{0}$ with the quotient topology from $\mathscr{H}_{ \pm}$or from $\mathscr{H}_{\cup}$ (both topologies on $\mathscr{H}_{ \pm}^{0}$ coincide) and with the representations $\rho_{ \pm}$of $\mathbb{R}_{+}^{\times}$induced by $\lambda$ on $\mathscr{H}_{ \pm}$ or $\mathscr{H}_{\cup}$. We view the pair of representations $\left(\rho_{+}, \rho_{-}\right)$as a formal difference $\rho_{+} \ominus \rho_{-}$, that is, as a virtual representation of $\mathbb{R}_{+}^{\times}$.

A smooth representation of $\mathbb{R}_{+}^{\times} \cong \mathbb{R}$ is determined uniquely by the action of the generator of the Lie algebra of $\mathbb{R}_{+}^{\times}$. This generator corresponds to the scaling invariant vector field $D f(x)=x f^{\prime}(x)$ on $\mathscr{H} \cup$. We let $D_{ \pm}$be the operators on $\mathscr{H}_{ \pm}^{0}$ induced by $D$ on $\mathscr{H}_{\cup}$. We claim that the operators $D_{ \pm}$are spectral interpretations for the poles and zeros of the complete $\zeta$-function $\xi$ defined in (1). This is rather trivial for $D_{+}$. Since

$$
\mathscr{H}_{+}^{0} \cong \mathbb{C}\left(x^{0}\right) \oplus \mathbb{C}\left(x^{1}\right),
$$


the operator $D_{+}$is equivalent to the diagonal $2 \times 2$-matrix with eigenvalues 0 , 1. Hence it is a spectral interpretation for the two poles of $\xi$. To treat $\mathscr{H}_{-}^{0}$, we identify the Fourier-Laplace transforms of $\mathscr{H}_{-}$and $Z \mathscr{H}_{\cap}$. Recall that $\hat{f}(s)=$ $\int_{0}^{\infty} f(x) x^{s} d^{\times} x$.

Theorem 4.1. The operator $f \mapsto \hat{f}$ identifies $\mathscr{H}_{-}$with the space of entire functions $h: \mathbb{C} \rightarrow \mathbb{C}$ for which $t \mapsto h(s+\mathrm{i} t)$ is a Schwartz function on $\mathbb{R}$ for each $s \in \mathbb{R}$.

The subspace $Z \mathscr{H}_{\cap}$ is mapped to the space of those entire functions $h$ for which

$$
t \mapsto \frac{h(s+\mathrm{i} t)}{\zeta(s+\mathrm{i} t)} \quad \text { and } \quad t \mapsto \frac{h(s+\mathrm{i} t)}{\zeta(1-s-\mathrm{i} t)}
$$

are Schwartz functions for $s \geq 1 / 2$ and $s \leq 1 / 2$, respectively. (In particular, this means that $h(z) / \zeta(z)$ has no poles with $\operatorname{Re} z \geq 1 / 2$ and $h(z) / \zeta(1-z)$ has no poles with $\operatorname{Re} z \leq 1 / 2$.)

Proof. It is well-known that the Fourier transform is an isomorphism (of topological vector spaces) $\mathscr{S}\left(\mathbb{R}_{+}^{\times}\right) \cong \mathscr{S}(\mathrm{i} \mathbb{R})$. Hence $f \mapsto \hat{f}$ is an isomorphism $\mathscr{S}\left(\mathbb{R}_{+}^{\times}\right)_{s} \cong \mathscr{S}(s+\mathrm{i} \mathbb{R})$ for all $s \in \mathbb{R}$. It is clear that $\hat{f}$ is an entire function on $\mathbb{C}$ for $f \in \mathscr{H}_{-}$. Since $\mathscr{H}_{-}=\bigcap_{s \in \mathbb{R}} \mathscr{S}\left(\mathbb{R}_{+}^{\times}\right)_{s}$, we also get $\hat{f} \in \mathscr{S}(s+\mathrm{i} \mathbb{R})$ for all $s \in \mathbb{R}$. Conversely, if $h$ is entire and $h \in \mathscr{S}(s+\mathrm{i} \mathbb{R})$ for all $s \in \mathbb{R}$, then for each $s \in \mathbb{R}$ there is $f_{s} \in \mathscr{S}\left(\mathbb{R}_{+}^{\times}\right)_{s}$ with $h(s+\mathrm{i} t)=\widehat{f}_{s}(s+\mathrm{i} t)$ for all $t \in \mathbb{R}$. Using the Cauchy-Riemann differential equation for the analytic function $h(s+\mathrm{i} t)$, we conclude that $f_{s}$ is independent of $s$. Hence we get a function $f$ in $\mathscr{H}_{-}$with $\hat{f}=h$ on all of $\mathbb{C}$. This yields the desired description of $\left(\mathscr{H}_{-}\right) \hat{\text {. }}$

The same argument shows that $\mathscr{S}\left(\mathbb{R}_{+}^{\times}\right) \widehat{I}$ for an open interval $I$ is the space of all holomorphic functions $h: I+\mathrm{i} \mathbb{R} \rightarrow \mathbb{C}$ with $h \in \mathscr{S}(s+\mathrm{i} \mathbb{R})$ for all $s \in I$.

Proposition 2.1 asserts that $f \in \mathscr{H}_{+}$if and only if $f \in \mathscr{S}\left(\mathbb{R}_{+}^{\times}\right)_{0, \infty}[$ and $J \mathfrak{F} f \in \mathscr{S}\left(\mathbb{R}_{+}^{\times}\right)_{]-\infty, 1[}$. Since $J \mathfrak{F}$ is invertible on $\mathscr{S}\left(\mathbb{R}_{+}^{\times}\right)_{s}$ for $0<s<1$, this is equivalent to $f \in \mathscr{S}\left(\mathbb{R}_{+}^{\times}\right)_{s}$ for $s \geq 1 / 2$ and $J \mathfrak{F} f \in \mathscr{S}\left(\mathbb{R}_{+}^{\times}\right)_{s}$ for $s \leq 1 / 2$. Moreover, these two conditions are equivalent for $s=1 / 2$. We get $f \in Z \mathscr{H}_{+}$if and only if $Z^{-1} f \in \mathscr{S}\left(\mathbb{R}_{+}^{\times}\right)_{s}$ for $s \geq 1 / 2$ and $J \mathfrak{F} Z^{-1} f \in \mathscr{S}\left(\mathbb{R}_{+}^{\times}\right)_{s}$ for $s \leq 1 / 2$.

Now let $h: \mathbb{C} \rightarrow \mathbb{C}$ be an entire function with $h \in \mathscr{S}(s+\mathrm{i} \mathbb{R})$ for all $s \in \mathbb{R}$. Thus $h=\hat{f}$ for some $f \in \mathscr{H}_{-}$. Equation (13) implies $f \in Z \mathscr{H}_{\cap}$ if and only if $h / \zeta \in \mathscr{S}(s+\mathrm{i} \mathbb{R})$ for $s \geq 1 / 2$ and $(J \mathfrak{F} f)^{\wedge} / \zeta \in \mathscr{S}(s+\mathrm{i} \mathbb{R})$ for $s \leq 1 / 2$. The functional equation (14) yields $(J \mathfrak{F} f)\}(z) / \zeta(z)=h(z) / \zeta(1-z)$.

Let $\left(\mathscr{H}_{-}^{0}\right)^{\prime}$ be the space of continuous linear functionals $\mathscr{H}_{-}^{0} \rightarrow \mathbb{C}$ and let ${ }^{t} D_{-} \in \operatorname{End}\left(\left(\mathscr{H}_{-}^{0}\right)^{\prime}\right)$ be the transpose of $D_{-}$. 
Corollary 4.2. The eigenvalues of the transpose ${ }^{t} D_{-} \in \operatorname{End}\left(\left(\mathscr{H}_{-}^{0}\right)^{\prime}\right)$ are exactly the zeros of the complete Riemann $\zeta$-function $\xi$. The algebraic multiplicity of $\lambda$ as an eigenvalue of ${ }^{t} D_{-}$is the zero order of $\xi$ at $\lambda$. Here we define the algebraic multiplicity as the dimension of $\bigcup_{k \in \mathbb{N}} \operatorname{ker}\left({ }^{t} D_{-}-\lambda\right)^{k}$.

Proof. We can identify $\left(\mathscr{H}_{-}^{0}\right)^{\prime}$ with the space of continuous linear functionals on $\mathscr{H}_{-}$that annihilate $Z \mathscr{H}_{\cap}$. Let $l: \mathscr{H}_{-} \rightarrow \mathbb{C}$ be such a linear functional. In the Fourier-Laplace transformed picture, we have $D_{-} h(s)=s \cdot h(s)$ for all $s \in \mathbb{C}$. Hence $l$ is an eigenvector for the transpose ${ }^{t} D_{-}$if and only if it is of the form $h \mapsto h(s)$ for some $s \in \mathbb{C}$. Similarly, $\left({ }^{t} D_{-}-s\right)^{k}(l)=0$ if and only if $l(h)=\sum_{j=0}^{k-1} a_{j} h^{(j)}(s)$ for some $a_{0}, \ldots, a_{k-1} \in \mathbb{C}$. It follows from the functional equation $\xi(s)=\xi(1-s)$ that the zero orders of $\xi$ at $s$ and $1-s$ agree for all $s \in \mathbb{C}$. Moreover, $\xi$ and $\zeta$ have the same zeros and the same zero orders for Re $s>0$. Hence the assertion follows from Theorem 4.1.

\section{A geometric character computation}

Our next goal is to prove that the representation $\rho$ is summable and to compute its character geometrically.

Definition 5.1 ( $[$ Mey $]$ ). Let $G$ be a Lie group and let $\mathscr{D}(G)$ be the space of smooth, compactly supported functions on $G$. A smooth representation $\rho$ of $G$ on a Fréchet space is called summable if $\int \rho(f)$ is nuclear for all $f \in \mathscr{D}(G)$ and if these operators are uniformly nuclear for $f$ in a bounded subset of $\mathscr{D}(G)$.

The theory of nuclear operators is due to Alexandre Grothendieck and rather deep. Nuclear operators are analogues of trace class operators on Hilbert spaces. It follows easily from the definition that $f \circ g$ is nuclear if at least one of the operators $f$ and $g$ is nuclear. That is, the nuclear operators form an operator ideal. For the purposes of this article, we do not have to recall the definition of nuclearity because of the following simple criterion:

Theorem 5.2. An operator between nuclear Fréchet spaces is nuclear if and only if it can be factored through a Banach space.

The spaces $\mathscr{S}\left(\mathbb{R}_{+}^{\times}\right)_{I}$ and $\mathscr{H}_{+}$are nuclear because $\mathscr{S}(\mathbb{R})$ is nuclear and nuclearity is hereditary for subspaces and inverse limits. Hence Theorem 5.2 applies to all operators between these spaces.

The character of a summable representation $\rho$ is the distribution on $G$ defined by $\chi_{\rho}(f):=\operatorname{tr} \int \rho(f)$ for all $f \in \mathscr{D}(G)$. The uniform nuclearity of $\int \rho(f)$ for $f$ in bounded subsets of $\mathscr{D}(G)$ ensures that $\chi_{\rho}$ is a bounded linear functional on $\mathscr{D}(G)$. This is equivalent to continuity because $\mathscr{D}(G)$ is an LF-space. 
If $\rho$ is a virtual representation as in our case, we let $\operatorname{tr} \int \rho(f)$ be the supertrace $\operatorname{tr} \int \rho_{+}(f)-\operatorname{tr} \int \rho_{-}(f)$.

One can see from the above arguments and definitions that summability of representations really has to do with bounded subsets of $\mathscr{D}(G)$ and bounded maps, not with open subsets and continuous maps. The same is true for the concept of a nuclear operator. That is, the theory of nuclear operators and summable representations is at home in bornological vector spaces. We can still give definitions in the context of topological vector spaces if we turn them into bornological vector spaces using the standard bornology of (von Neumann) bounded subsets. Nevertheless, topological vector spaces are the wrong setup for studying nuclearity. The only reason why I use them here is because they are more familiar to most readers and easier to find in the literature.

We need uniform nuclearity because we want $\chi_{\rho}(f)$ to be a bounded linear functional of $f$. In the following, we will only prove nuclearity of various operators. The same proofs yield uniform nuclearity as well. We leave it to the reader to add the remaining details. Suffice it to say that there are analogues of Theorem 5.2 and Theorem 5.5 below for uniformly nuclear sets of operators.

In order to prove the summability of our spectral interpretation $\rho$, we define several operators between $\mathscr{H}_{ \pm}$and the space $\mathscr{S}_{>} \oplus \mathscr{S}_{<}$. As auxiliary data, we use a smooth function $\varphi: \mathbb{R}_{+} \rightarrow[0,1]$ with $\varphi(t)=0$ for $t \ll 1$ and $\varphi(t)=1$ for $t \gg 1$. Let $M_{\varphi}$ be the operator of multiplication by $\varphi$. We assume for simplicity that $\varphi(t)+\varphi\left(t^{-1}\right)=1$, so that

$$
M_{\varphi}+J M_{\varphi} J=\mathrm{id}
$$

It is easy to check that $M_{\varphi}$ is a continuous map from $\mathscr{S}_{>}$into $\mathscr{O}\left(\mathbb{R}_{+}^{\times}\right)$. We warn the reader that our notation differs from that in $[\mathbf{M e y}]$ : there the auxiliary function $1-\varphi$ is used and denoted $\varphi$.

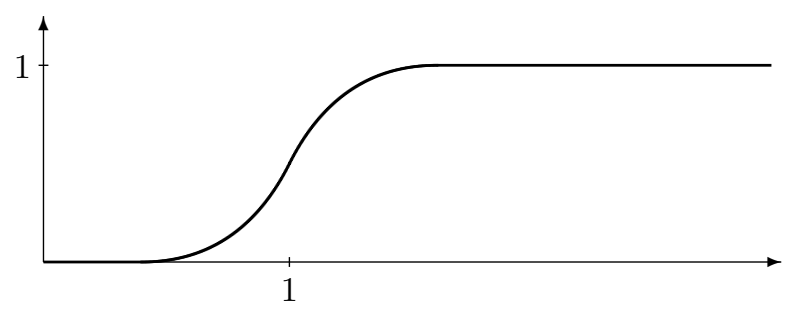

Figure 1. The auxiliary function $\varphi$ 
Now we can define our operators:

$$
\begin{array}{rlrl}
\iota_{+}: \mathscr{H}_{+} \rightarrow \mathscr{S}_{>} \oplus \mathscr{S}_{<}, & \iota_{+} f & :=(Z f, J Z \mathfrak{F} f) \\
\iota_{-}: \mathscr{H}_{-} \rightarrow \mathscr{S}_{>} \oplus \mathscr{S}_{<}, & \iota_{-} f & :=(f, f) ; \\
\pi_{+}: \mathscr{S}_{>} \oplus \mathscr{S}_{<} \rightarrow \mathscr{H}_{+}, & \pi_{+}\left(f_{1}, f_{2}\right):=\left(M_{\varphi} Z^{-1} f_{1}, \mathfrak{F} M_{\varphi} Z^{-1} J f_{2}\right) ; \\
\pi_{-}: \mathscr{S}_{>} \oplus \mathscr{S}_{<} \rightarrow \mathscr{H}_{-}, & \pi_{-}\left(f_{1}, f_{2}\right):=\left(M_{\varphi} f_{1}, J M_{\varphi} J f_{2}\right) .
\end{array}
$$

It follows from Equation (8) and Proposition 3.2 that these operators are welldefined and continuous. The operators $\iota_{ \pm}$are $\lambda$-equivariant, the operators $\pi_{ \pm}$ are not. We compute

$$
\begin{aligned}
\pi_{-} \iota_{-} & =M_{\varphi}+J M_{\varphi} J=\operatorname{id}_{\mathscr{H}_{-}}, \\
\pi_{+} \iota_{+}= & M_{\varphi}+\mathfrak{F} M_{\varphi} \mathfrak{F}=\operatorname{id}_{\mathscr{H}_{+}}+M_{\varphi}-\mathfrak{F} J M_{\varphi} J \mathfrak{F}, \\
\iota_{-} \pi_{-}= & \left(\begin{array}{cc}
M_{\varphi} & J M_{\varphi} J \\
M_{\varphi} & J M_{\varphi} J
\end{array}\right) \\
\iota_{+} \pi_{+}= & \left(\begin{array}{cc}
Z M_{\varphi} Z^{-1} & Z \mathfrak{F} M_{\varphi} Z^{-1} J \\
J Z \mathfrak{F} M_{\varphi} Z^{-1} & J Z M_{\varphi} Z^{-1} J
\end{array}\right) .
\end{aligned}
$$

Thus $\pi_{-}$is a section for $\iota_{-}$and $\iota_{-} \pi_{-} \in \operatorname{End}\left(\mathscr{S}_{>} \oplus \mathscr{S}_{<}\right)$is a projection onto a subspace isomorphic to $\mathscr{H}_{-}$. The proof of Theorem 3.3 shows that $\iota_{+}$has closed range and is a topological isomorphism onto its range. Although $\pi_{+}$is not a section for $\iota_{+}$, it is a near enough miss for the following summability arguments. I do not know whether there exists an honest section for $\iota_{+}$, that is, whether the range of $\iota_{+}$is a complemented subspace of $\mathscr{S}_{>} \oplus \mathscr{S}_{<}$.

Lemma 5.3. The operator $\int \lambda(f)\left(M_{\varphi}-Z M_{\varphi} Z^{-1}\right)$ on $\mathscr{S}_{>}$is nuclear for $f \in \mathscr{S}_{>}$.

The operator $\int \lambda(f) M_{\varphi}: \mathscr{S}_{>} \rightarrow \mathscr{S}_{<}$is nuclear for $f \in \mathscr{S}_{<}$.

The operator $\int \lambda(f)\left(M_{\varphi}-\mathfrak{F} J M_{\varphi} J \mathfrak{F}\right)$ on $\mathscr{H}_{+}$is nuclear for $f \in \mathscr{O}\left(\mathbb{R}_{+}^{\times}\right)$.

Proof. We have $\int \lambda(f) Z(h)=f * \check{\zeta} * h=(Z f) * h=\int \lambda(Z f)(h)$ for all $f, h \in \mathscr{S}_{>}$. Hence

$$
\begin{aligned}
\int \lambda(f)\left(M_{\varphi}-Z M_{\varphi} Z^{-1}\right)=\left[\int \lambda(f), M_{\varphi}\right]- & {\left[\int \lambda(f) Z, M_{\varphi}\right] Z^{-1} } \\
& =\left[\int \lambda(f), M_{\varphi}\right]-\left[\int \lambda(Z f), M_{\varphi}\right] Z^{-1}
\end{aligned}
$$

We are going to show that $\left[\int \lambda(f), M_{\varphi}\right]$ is a nuclear operator on $\mathscr{S}_{>}$for all $f \in \mathscr{S}_{>}$. Together with the above computation, this implies the first assertion of the lemma because $Z^{-1}$ is continuous on $\mathscr{S}_{>}$and $f, Z f \in \mathscr{S}_{>}$. 
One checks easily that

$$
\left[\int \lambda(f), M_{\varphi}\right](h)(x)=\int_{0}^{\infty} f\left(x y^{-1}\right)(\varphi(y)-\varphi(x)) h(y) d^{\times} y .
$$

Thus our operator has the smooth integral kernel $f\left(x y^{-1}\right)(\varphi(y)-\varphi(x))$. If $f$ had compact support, this integral kernel would also be compactly supported. If only $f \in \mathscr{S}_{>}$, we can still estimate that our kernel lies in $\mathscr{S}_{>} \hat{\otimes} \mathscr{S}\left(\mathbb{R}_{+}^{\times}\right)_{-s}$ for any $s \in] 1, \infty\left[\right.$. Thus $\left[\int \lambda(f), M_{\varphi}\right]$ factors through the embedding $\mathscr{S}_{>} \subseteq$ $L^{2}\left(\mathbb{R}_{+}^{\times}, x^{2 s} d^{\times} x\right)$ for any $s>1$. This implies nuclearity by Theorem 5.2 and finishes the proof of the first assertion of the lemma.

To prove the second assertion, we let $L_{I}^{2}=\bigcap_{s \in I} L^{2}\left(\mathbb{R}_{+}^{\times}, x^{2 s} d^{\times} x\right)$. This is a Fréchet space. We claim that $\int \lambda(f)$ is a continuous linear operator $L_{I}^{2} \rightarrow$ $\mathscr{S}_{I}$ for any open interval $I$. (Actually, $\mathscr{S}_{I}$ is the Gårding subspace for the representation $\lambda$ on $L_{I}^{2}$.) This follows from the description of $\mathscr{S}_{I}$ in the proof of Proposition 2.1 and $D^{m}(f * h)=\left(D^{m} f\right) * h$. Therefore, we have continuous linear operators

$$
\mathscr{S}_{>} \prec L_{s}^{2} \stackrel{M_{\varphi}}{\longrightarrow} L_{]-\infty, s]}^{2} \prec L_{]-\infty, 0[}^{2} \stackrel{\int \lambda(f)}{\longrightarrow} \mathscr{S}_{<}
$$

for any $s>1$. Thus $\int \lambda(f) M_{\varphi}$ factors through the Hilbert space $L_{s}^{2}$. This yields the assertion by our criterion for nuclear operators, Theorem 5.2.

We claim that $\int \lambda(f)\left(M_{\varphi}-\mathfrak{F} J M_{\varphi} J \mathfrak{F}\right)$ as an operator on $\mathscr{H}_{+}$factors continuously through $L_{1 / 2}^{2} \cong L^{2}\left(\mathbb{R}_{+}^{\times}, x d^{\times} x\right)$. This implies the third assertion. To prove the claim, we use $\mathscr{H}_{+}=\mathscr{S}_{>} \cap \mathfrak{F} J\left(\mathscr{S}_{<}\right)$. That is, the map

$$
\mathscr{H}_{+} \rightarrow \mathscr{S}_{>} \oplus \mathscr{S}_{<}, \quad f \mapsto(f, J \mathfrak{F} f)
$$

is a topological isomorphism onto its range. We have already seen this during the proof of Theorem 3.3. Hence we merely have to check the existence of continuous extensions

$\int \lambda(f)\left(M_{\varphi}-\mathfrak{F} J M_{\varphi} J \mathfrak{F}\right): L_{1 / 2}^{2} \rightarrow \mathscr{S}_{>}, \quad J \mathfrak{F} \circ \int \lambda(f)\left(M_{\varphi}-\mathfrak{F} J M_{\varphi} J \mathfrak{F}\right): L_{1 / 2}^{2} \rightarrow \mathscr{S}_{<}$.

We write

$$
\begin{aligned}
\int \lambda(f)\left(M_{\varphi}-\mathfrak{F} J M_{\varphi} J \mathfrak{F}\right)=-\int \lambda(f)\left(M_{1-\varphi}\right. & \left.-\mathfrak{F} J M_{1-\varphi} J \mathfrak{F}\right) \\
& =-\int \lambda(f) M_{1-\varphi}+\int \lambda(\mathfrak{F} J f) M_{1-\varphi} J \mathfrak{F} .
\end{aligned}
$$

Proposition 2.1 yields $\mathfrak{F} J f \in \mathscr{S}_{>}$. Moreover, $J$ and $\mathfrak{F}$ are unitary on $L_{1 / 2}^{2}$. Hence we get bounded extensions $L_{1 / 2}^{2} \rightarrow \mathscr{S}_{>}$of both summands by the same argument as for the second assertion of the lemma. Similarly, both summands in

$$
J \mathfrak{F} \circ \int \lambda(f)\left(M_{\varphi}-\mathfrak{F} J M_{\varphi} J \mathfrak{F}\right)=\int \lambda(J \mathfrak{F} f) M_{\varphi}-\int \lambda(f) M_{\varphi} J \mathfrak{F}
$$


have bounded extensions $L_{1 / 2}^{2} \rightarrow \mathscr{S}_{<}$as desired.

Corollary 5.4. The operator $\int \lambda(f) \circ\left(\iota_{-} \pi_{-}-\iota_{+} \pi_{+}\right)$on $\mathscr{S}_{>} \oplus \mathscr{S}_{<}$is nuclear for all $f \in \mathscr{O}\left(\mathbb{R}_{+}^{\times}\right)$.

The operator $\int \lambda(f) \circ\left(\mathrm{id}-\pi_{+} \iota_{+}\right)$on $\mathscr{H}_{+}$is nuclear for all $f \in \mathscr{O}\left(\mathbb{R}_{+}^{\times}\right)$.

Proof. We have

$$
\begin{aligned}
\int \lambda(f) \circ\left(\iota_{-} \pi_{-}-\iota_{+} \pi_{+}\right) & \\
& =\left(\begin{array}{cc}
\int \lambda(f)\left(M_{\varphi}-Z M_{\varphi} Z^{-1}\right) & \int \lambda(f) J\left(M_{\varphi}-J Z \mathfrak{F} M_{\varphi} Z^{-1}\right) J \\
\int \lambda(f)\left(M_{\varphi}-J Z \mathfrak{F} M_{\varphi} Z^{-1}\right) & \int \lambda(f) J\left(M_{\varphi}-Z M_{\varphi} Z^{-1}\right) J
\end{array}\right) .
\end{aligned}
$$

The upper left corner is nuclear by the first assertion of Lemma 5.3. Since

$$
\int \lambda(f)\left(J\left(M_{\varphi}-Z M_{\varphi} Z^{-1}\right) J\right)=J\left(\int \lambda(J f)\left(M_{\varphi}-Z M_{\varphi} Z^{-1}\right)\right) J,
$$

we also get the nuclearity of the lower right corner. We have $\int \lambda(f) J Z F=$ $\int \lambda(J Z \mathfrak{F} f)$ because $J Z \mathfrak{F}$ is $\lambda$-invariant. Proposition 2.1 and Proposition 3.2 yield $J Z \mathfrak{F}(f) \in \mathscr{S}_{<}$. Hence the two summands $\int \lambda(f) M_{\varphi}$ and $\int \lambda(f) J Z \mathfrak{F} M_{\varphi} Z^{-1}$ in the lower left corner are nuclear by the second assertion of Lemma 5.3. The assertion for the upper right corner follows by a symmetric argument. The nuclearity of $\int \lambda(f) \circ\left(\mathrm{id}-\pi_{+} \iota_{+}\right)$on $\mathscr{H}_{+}$is exactly the third assertion of Lemma 5.3.

In order to apply this to the representation $\rho$, we need a general fact about nuclear operators. Let $W_{1}, W_{2}$ be Fréchet spaces and let $V_{1} \subseteq W_{1}$ and $V_{2} \subseteq W_{2}$ be closed subspaces. If $T: W_{1} \rightarrow W_{2}$ maps $V_{1}$ into $V_{2}$, we write $\left.T\right|_{V_{1}, V_{2}}$ and $\left.T\right|^{W_{1} / V_{1}, W_{2} / V_{2}}$ for the operators $V_{1} \rightarrow V_{2}$ and $W_{1} / V_{1} \rightarrow W_{2} / V_{2}$ induced by $T$.

Theorem 5.5 ([Gro55]). If $T$ is nuclear, so are $\left.T\right|_{V_{1}, V_{2}}$ and $\left.T\right|^{W_{1} / V_{1}, W_{2} / V_{2}}$. If $V_{1}=V_{2}=V$ and $W_{1}=W_{2}=W$, then $\operatorname{tr} T=\left.\operatorname{tr} T\right|_{V}+\left.\operatorname{tr} T\right|^{W / V}$.

Proposition 5.6. The representation $\rho: \mathbb{R}_{+}^{\times} \rightarrow \operatorname{Aut}\left(\mathscr{H}^{0}\right)$ is summable and

$$
\chi(\rho)(f)=\operatorname{tr} \int \lambda(f)\left(\mathrm{id}-\pi_{+} \iota_{+}\right)-\operatorname{tr} \int \lambda(f)\left(\iota_{-} \pi_{-}-\iota_{+} \pi_{+}\right) .
$$

Proof. The embeddings of $\mathscr{H}_{+}$and $\mathscr{H}_{-}$in $\mathscr{S}_{>} \oplus \mathscr{S}_{<}$agree on the common subspace $\mathscr{H}_{\cap} \cong Z \mathscr{H}_{\cap}$ and hence combine to an embedding of $\mathscr{H}_{\cup}$. Thus we identify $\mathscr{H}_{\cup}$ with the subspace $\iota_{+} \mathscr{H}_{+}+\iota_{-} \mathscr{H}_{-}$of $\mathscr{S}_{>} \oplus \mathscr{S}_{<}$. Let $T:=$ $\int \lambda(f)\left(\iota_{-} \pi_{-}-\iota_{+} \pi_{+}\right)$. The range of $T$ is contained in $\mathscr{H}_{\cup}$. Therefore, its trace as an operator on $\mathscr{S}_{>} \oplus \mathscr{S}_{<}$agrees with its trace as an operator on $\mathscr{H}_{\cup}$ by Theorem 5.5. Write

$$
\left.T\right|_{\mathscr{H}}=\int \lambda(f)\left(\operatorname{id}_{\mathscr{H}}-\left.\iota_{+} \pi_{+}\right|_{\mathscr{H}}\right)-\int \lambda(f)\left(\operatorname{id}_{\mathscr{H}}-\left.\iota_{-} \pi_{-}\right|_{\mathscr{H}}\right) .
$$


Since $\iota_{-} \pi_{-}$is a projection onto $\mathscr{H}_{-} \subset \mathscr{H}_{\cup}$, Theorem 5.5 yields

$$
\operatorname{tr} \int \lambda(f)\left(\operatorname{id}_{\mathscr{H}}-\iota_{-} \pi_{-} \mid \mathscr{H}_{\cup}\right)=\left.\operatorname{tr} \int \lambda(f)\right|^{\mathscr{H}_{\cup} / \mathscr{H}_{-}}=\chi\left(\rho_{+}\right)(f) .
$$

Similarly, since $\iota_{+} \pi_{+}$maps $\mathscr{H} \cup$ into $\mathscr{H}_{+}$we get

$$
\begin{array}{r}
\operatorname{tr} \int \lambda(f)\left(\operatorname{id} \mathscr{H}_{\iota}-\iota_{+} \pi_{+} \mid \mathscr{H}_{\iota}\right)=\left.\operatorname{tr} \int \lambda(f)\right|^{\mathscr{H}_{\iota} / \mathscr{H}_{+}}+\operatorname{tr} \int \lambda(f)\left(\operatorname{id} \mathscr{H}_{+}-\iota_{+} \pi_{+} \mid \mathscr{H}_{+}\right) \\
=\chi\left(\rho_{-}\right)(f)+\operatorname{tr} \int \lambda(f)\left(\operatorname{id}_{\mathscr{H}_{+}}-\pi_{+} \iota_{+}\right) .
\end{array}
$$

Hence

$$
\operatorname{tr} \int \lambda(f)\left(\iota_{-} \pi_{-}-\iota_{+} \pi_{+}\right)=\chi\left(\rho_{-}\right)(f)+\operatorname{tr} \int \lambda(f)\left(\operatorname{id} \mathscr{H}_{+}-\pi_{+} \iota_{+}\right)-\chi\left(\rho_{+}\right)(f) .
$$

Along the way, we see that the operators whose trace we take are nuclear. That is, $\rho_{+}$and $\rho_{-}$are summable representations.

It remains to compute the traces in Proposition 5.6 explicitly. We need the following definitions. For a continuous function $f: \mathbb{R}_{+}^{\times} \rightarrow \mathbb{C}$, let $\tau(f):=f(1)$ and $\partial f(x)=f(x) \ln x$. This defines a bounded derivation $\partial$ on $\mathscr{S}\left(\mathbb{R}_{+}^{\times}\right)_{I}$ for any interval $I$, that is, $\partial\left(f_{1} * f_{2}\right)=\partial\left(f_{1}\right) * f_{2}+f_{1} * \partial\left(f_{2}\right)$. This derivation is the generator of the dual action $t \cdot f(x):=x^{\mathrm{it}} f$ of $\mathbb{R}$. Notice that $\tau(\partial f)=0$. The obvious extension of $\partial$ to distributions is still a derivation.

Lemma 5.7. Let $f_{0}, f_{1} \in \mathscr{S}\left(\mathbb{R}_{+}^{\times}\right)_{s}$ for some $s \in \mathbb{R}$. Then $\int \lambda\left(f_{0}\right)\left[M_{\varphi}, \int \lambda\left(f_{1}\right)\right]$ is a nuclear operator on $L^{2}\left(\mathbb{R}_{+}^{\times}, x^{2 s} d^{\times} x\right)$ and $\mathscr{S}\left(\mathbb{R}_{+}^{\times}\right)_{s}$ and

$$
\operatorname{tr} \int \lambda\left(f_{0}\right)\left[M_{\varphi}, \int \lambda\left(f_{1}\right)\right]=\tau\left(f_{0} * \partial f_{1}\right) .
$$

Proof. The operators of multiplication by $x^{ \pm s}$ are unitary operators between $L_{s}^{2}$ and $L_{0}^{2}$. We can use them to reduce the general case to the special case $s=0$. We assume this in the following. We have checked above that $\left[M_{\varphi}, \int \lambda\left(f_{1}\right)\right]$ has an integral kernel in $\mathscr{S}\left(\mathbb{R}_{+}^{\times}\right) \hat{\otimes} \mathscr{S}\left(\mathbb{R}_{+}^{\times}\right)$. Therefore, so has $\int \lambda\left(f_{0}\right)\left[M_{\varphi}, \int \lambda\left(f_{1}\right)\right]$. This implies nuclearity as an operator from $L^{2}\left(\mathbb{R}_{+}^{\times}, d^{\times} x\right)$ to $\mathscr{S}\left(\mathbb{R}_{+}^{\times}\right)$by Theorem 5.2. Moreover, the operator has the same trace on both spaces. Explicitly, the integral kernel is

$$
(x, y) \mapsto \int_{0}^{\infty} f_{0}\left(x z^{-1}\right) f_{1}\left(z y^{-1}\right)(\varphi(z)-\varphi(y)) d^{\times} z .
$$

We get

$$
\begin{aligned}
\operatorname{tr} \int \lambda\left(f_{0}\right)\left[M_{\varphi}, \int \lambda\left(f_{1}\right)\right]= & \int_{0}^{\infty} \int_{0}^{\infty} f_{0}\left(x z^{-1}\right) f_{1}\left(z x^{-1}\right)(\varphi(z)-\varphi(x)) d^{\times} z d^{\times} x \\
= & \int_{0}^{\infty} f_{0}(x) f_{1}\left(x^{-1}\right) \int_{0}^{\infty} \varphi(z)-\varphi(x z) d^{\times} z d^{\times} x .
\end{aligned}
$$


We compute $\int_{0}^{\infty} \varphi(z)-\varphi(x z) d^{\times} z$. If $\varphi$ had compact support, the $\lambda$-invariance of $d^{\times} z$ would force the integral to vanish. Therefore, we may replace $\varphi$ by any function $\varphi^{\prime}$ with the same behaviour at 0 and $\infty$. We choose $\varphi^{\prime}$ to be the characteristic function of $\left[1, \infty\left[\right.\right.$. If $x \leq 1$, then $\varphi^{\prime}(z)-\varphi^{\prime}(x z)$ is the characteristic function of the interval $\left[1, x^{-1}\right.$ [, so that the integral is $\ln \left(x^{-1}\right)$. We get the same value for $x \geq 1$ as well. Hence $\operatorname{tr} \int \lambda\left(f_{0}\right)\left[M_{\varphi}, \int \lambda\left(f_{1}\right)\right]=$ $\int_{0}^{\infty} f_{0}(x) f_{1}\left(x^{-1}\right) \ln \left(x^{-1}\right) d^{\times} x=\tau\left(f_{0} * \partial f_{1}\right)$.

Theorem 5.8. Define the distributions $W_{p}$ for $p \in \mathscr{P}$ and $p=\infty$ as in (2) and (3). Then

$$
\sum_{z \in \mathbb{C}} \operatorname{ord}_{\xi}(z) \hat{f}(z)=\chi(\rho)(f)=\sum_{p \in \mathscr{P}} W_{p}(f)+W_{\infty}(f)
$$

for all $f \in \mathscr{O}\left(\mathbb{R}_{+}^{\times}\right)$. Here $\operatorname{ord}_{\xi}(z)$ denotes the order at $z$ of the complete $\zeta$-function $\xi$, which is positive at poles and negative at zeros of $\xi$.

Proof. The trace of a nuclear operator on a nuclear Fréchet space is equal to the sum of its eigenvalues counted with algebraic multiplicity (see [Gro55]). Since $\operatorname{tr}(A)=\operatorname{tr}\left({ }^{t} A\right)$ for any nuclear operator $A$, the first equality follows from Corollary 4.2. It remains to show $\chi(\rho)(f)=\sum_{p \in \mathscr{P}} W_{p}(f)+W_{\infty}(f)$. Proposition 5.6 yields

$$
\begin{array}{r}
\begin{aligned}
& \chi(\rho)(f)=-\operatorname{tr} \int \lambda(f)\left(\iota_{-} \pi_{-}-\iota_{+} \pi_{+}\right)+ \operatorname{tr} \int \lambda(f)\left(\mathrm{id}-\pi_{+} \iota_{+}\right) \\
&=-\left.\operatorname{tr} \int \lambda(f)\left(M_{\varphi}-Z M_{\varphi} Z^{-1}\right)\right|_{\mathscr{S}_{>}}-\left.\operatorname{tr} \int \lambda(f) J\left(M_{\varphi}-Z M_{\varphi} Z^{-1}\right) J\right|_{\mathscr{S}_{<}} \\
&-\left.\operatorname{tr} \int \lambda(f)\left(M_{\varphi}-\mathfrak{F} J M_{\varphi} J \mathfrak{F}\right)\right|_{\mathscr{H}_{+}} .
\end{aligned}
\end{array}
$$

It suffices to check that this agrees with $W(f)$ if $f=f_{0} * f_{1}$ because such elements are dense in $\mathscr{O}\left(\mathbb{R}_{+}^{\times}\right)$. We compute

$$
\begin{aligned}
-\left.\operatorname{tr} \int \lambda\left(f_{0} * f_{1}\right)\left(M_{\varphi}-Z M_{\varphi} Z^{-1}\right)\right|_{\mathscr{S}_{>}} & \\
=\operatorname{tr} \int \lambda\left(f_{0}\right) & {\left[M_{\varphi}, \int \lambda\left(f_{1}\right)\right]-\operatorname{tr} \int \lambda\left(f_{0}\right)\left[M_{\varphi}, \int \lambda\left(Z f_{1}\right)\right] Z^{-1} } \\
= & \operatorname{tr} \int \lambda\left(f_{0}\right)\left[M_{\varphi}, \int \lambda\left(f_{1}\right)\right]-\operatorname{tr} \int \lambda\left(Z^{-1} f_{0}\right)\left[M_{\varphi}, \int \lambda\left(Z f_{1}\right)\right]
\end{aligned}
$$

because $\operatorname{tr}(A B)=\operatorname{tr}(B A)$ if $A$ is nuclear. This is a nuclear operator $L_{s}^{2} \rightarrow \mathscr{S}_{>}$ for any $s>1$. Hence it has the same trace as an operator on $\mathscr{S}_{>}$and $L_{s}^{2}$. Lemma 5.7 yields

$$
\begin{array}{r}
-\left.\operatorname{tr} \int \lambda\left(f_{0} * f_{1}\right)\left(M_{\varphi}-Z M_{\varphi} Z^{-1}\right)\right|_{\mathscr{S}_{>}}=\tau\left(f_{0} * \partial f_{1}\right)-\tau\left(Z^{-1} f_{0} * \partial\left(Z f_{1}\right)\right) \\
=-\tau\left(f_{0} * f_{1} * Z^{-1} \partial(Z)\right)=\tau\left(f_{0} * f_{1} * Z \partial\left(Z^{-1}\right)\right)
\end{array}
$$


where $\partial(Z)$ is defined in the obvious way. We also use $\partial\left(Z^{-1}\right)=-Z^{-2} \partial(Z)$, which follows from the derivation property. Now we use the Euler product for the Zeta operator and the derivation rule:

$$
\begin{aligned}
Z * \partial\left(Z^{-1}\right)=Z * \partial\left(\prod_{p \in \mathscr{P}}\right. & \left.\left(1-\lambda_{p}^{-1}\right)\right)=\sum_{p \in \mathscr{P}}\left(1-\lambda_{p}^{-1}\right)^{-1} \partial\left(1-\lambda_{p}^{-1}\right) \\
=\sum_{p \in \mathscr{P}} \ln (p) \lambda_{p}^{-1}\left(1-\lambda_{p}^{-1}\right)^{-1} & =\sum_{p \in \mathscr{P}} \sum_{e=1}^{\infty} \ln (p) \lambda_{p}^{-e} .
\end{aligned}
$$

Hence

$$
-\left.\operatorname{tr} \int \lambda(f)\left(M_{\varphi}-Z M_{\varphi} Z^{-1}\right)\right|_{\mathscr{S}_{>}}=\tau\left(f * \sum_{p \in \mathscr{P}} \sum_{e=1}^{\infty} \ln (p) \lambda_{p}^{-e}\right)=\sum_{p \in \mathscr{P}} \sum_{e=1}^{\infty} \ln (p) f\left(p^{e}\right) .
$$

The second summand in (15) is reduced to this one by

$$
\begin{aligned}
\left.\operatorname{tr} \int \lambda(f) J\left(M_{\varphi}-Z M_{\varphi} Z^{-1}\right) J\right|_{\mathscr{S}_{<}}=\left.\operatorname{tr} J \int \lambda(f) J\left(M_{\varphi}-Z M_{\varphi} Z^{-1}\right)\right|_{\mathscr{S}_{>}} \\
=\left.\operatorname{tr} \int \lambda(J f)\left(M_{\varphi}-Z M_{\varphi} Z^{-1}\right)\right|_{\mathscr{S}_{>}} .
\end{aligned}
$$

Hence

$$
-\left.\operatorname{tr} \int \lambda(f) J\left(M_{\varphi}-Z M_{\varphi} Z^{-1}\right) J\right|_{\mathscr{S}_{<}}=\sum_{p \in \mathscr{P}} \sum_{e=1}^{\infty} \ln (p) p^{-e} f\left(p^{-e}\right) .
$$

These two summands together equal $\sum_{p \in \mathscr{P}} W_{p}$.

Now we treat the third summand in (15). The same arguments as above yield

$$
\begin{gathered}
\quad-\left.\operatorname{tr} \int \lambda\left(f_{0} * f_{1}\right)\left(M_{\varphi}-\mathfrak{F} J M_{\varphi} J \mathfrak{F}\right)\right|_{\mathscr{H}_{+}} \\
=\operatorname{tr} \int \lambda\left(f_{0}\right)\left[M_{\varphi}, \int \lambda\left(f_{1}\right)\right]-\operatorname{tr} \int \lambda\left(f_{0}\right)\left[M_{\varphi}, \int \lambda\left(\mathfrak{F} J f_{1}\right)\right] J \mathfrak{F} \\
=\tau\left(f_{0} * \partial f_{1}\right)-\tau\left(J \mathfrak{F}\left(f_{0}\right) * \partial\left(\mathfrak{F} J f_{1}\right)\right)=-\tau\left(f_{0} * f_{1} * J \mathfrak{F} \partial(\mathfrak{F} J)\right)=\tau\left(\mathfrak{F} J \partial\left(J \mathfrak{F}\left(f_{0} * f_{1}\right)\right)\right) .
\end{gathered}
$$

Here we use $\tau(\partial f)=0$. Explicitly,

$\tau(\mathfrak{F} J \partial(J \mathfrak{F} f))=\mathfrak{F} M_{\ln \left(x^{-1}\right)} \mathfrak{F}^{-1} f(1)=-\mathfrak{F}(\ln x) \dagger f(1)=-\langle\mathfrak{F}(\ln x), y \mapsto f(1-y)\rangle$.

Here $M_{\ln \left(x^{-1}\right)}$ denotes the operator of multiplication by $\ln \left(x^{-1}\right)=-\ln x$ and $\dagger$ denotes convolution with respect to the additive structure on $\mathbb{R}$. Thus it remains to compute the Fourier transform of $\ln x$. Since $\psi \mapsto \int_{\mathbb{R}} \psi(x) \ln x d x$ defines a tempered distribution on $\mathbb{R}, \mathfrak{F}(\ln x)$ is a well-defined tempered distribution on $\mathbb{R}$. The covariance property $\ln (t x)=\ln (t)+\ln (x)$ for $t, x \in \mathbb{R}_{+}^{\times}$ implies

$$
\left\langle\mathfrak{F}(\ln x), \lambda_{t} \psi\right\rangle=\langle\mathfrak{F}(\ln x), \psi\rangle-\ln (t) \psi(0)
$$


Especially, $\mathfrak{F}(\ln x)$ is $\lambda$-invariant on the space of $\psi \in \mathscr{S}(\mathbb{R})$ with $\psi(0)=0$. Thus

$$
\langle\mathfrak{F}(\ln x), \psi\rangle=c \int_{\mathbb{R}^{\times}} \psi(x) d^{\times} x
$$

for some constant $c \in \mathbb{R}$ for all $\psi \in \mathscr{S}(\mathbb{R})$ with $\psi(0)=0$.

We claim that $c=-1$. To see this, pick $\psi \in \mathscr{S}(\mathbb{R})$ with $\psi(0) \neq 0$ and consider $\psi-\lambda_{t} \psi$ for some $t \neq 1$. Equation (16) yields

$$
c \int_{\mathbb{R} \times} \psi(x)-\psi\left(t^{-1} x\right) d^{\times} x=\ln (t) \psi(0) .
$$

As in the proof of Lemma 5.7, this implies $c=-1$. Thus the distribution $\mathfrak{F}(\ln x)$ is some principal value for the integral $-\int_{\mathbb{R}^{\times}} \psi(x)|x|^{-1} d x$. This principal value can be described uniquely by the condition that $\mathfrak{F}^{2}(\ln x)(1)=0$. See also [Con99] for a comparison between this principal value and the one that usually occurs in the explicit formulas.

Finally, we compute

$$
\begin{gathered}
-\left.\operatorname{tr} \int \lambda(f)\left(M_{\varphi}-\mathfrak{F} J M_{\varphi} J \mathfrak{F}\right)\right|_{\mathscr{H}_{+}}=-\langle\mathfrak{F}(\ln x), y \mapsto f(1-y)\rangle=p v \int_{\mathbb{R}^{\times}} f(1-y) \frac{d y}{|y|} \\
=p v \int_{-\infty}^{\infty} f(x) \frac{d x}{|1-x|}=p v \int_{0}^{\infty} \frac{f(x)}{|1-x|}+\frac{f(x)}{1+x} d x=W_{\infty}(f) .
\end{gathered}
$$

Plugging this into (15), we get the desired formula for $\chi(\rho)$.

\section{Generalisation to Dirichlet $L$-functions}

We recall the definition of Dirichlet $L$-functions. Fix some $d \in \mathbb{N}_{\geq 2}$ and let $(\mathbb{Z} / d \mathbb{Z})^{\times}$be the group of invertible elements in the finite ring $\mathbb{Z} / d \mathbb{Z}$. Let $\chi$ be a character of $(\mathbb{Z} / d \mathbb{Z})^{\times}$. Define $\chi: \mathbb{N} \rightarrow \mathbb{C}$ by $\chi(n):=\chi(n \bmod d)$ if $(n, d)=1$ and $\chi(n):=0$ otherwise. The associated Dirichlet $L$-function is defined by

$$
L_{\chi}(s):=\sum_{n=1}^{\infty} \frac{\chi(n)}{n^{s}} .
$$

We suppose that $d$ is equal to the conductor of $\chi$, that is, $\chi$ does not factor through $\left(\mathbb{Z} / d^{\prime} \mathbb{Z}\right)^{\times}$for any proper divisor $d^{\prime} \mid d$. In particular, $\chi \neq 1$.

The constructions for the Riemann $\zeta$-function that we have done above work similarly for such $L$-functions. We define the space $\mathscr{H}_{-}$as above and let

$$
\mathscr{H}_{+}:=\{f \in \mathscr{S}(\mathbb{R}) \mid f(-x)=\chi(-1) f(x)\}
$$

be the space of even or odd functions, depending on $\chi(-1) \in\{ \pm 1\}$. The Fourier transform on $\mathscr{S}(\mathbb{R})$ preserves the subspace $\mathscr{H}_{+}$, and the assertions of 
Proposition 2.1 remain true. However, now $\mathfrak{F}^{2}=\chi(-1)$, so that we have to replace $\mathfrak{F}$ by $\mathfrak{F}^{*}=\chi(-1) \mathfrak{F}$ in appropriate places to get correct formulas.

Of course, the $L$-function analogue of the Zeta operator is defined by

$$
\mathscr{L}_{\chi} f(x):=\sum_{n=1}^{\infty} \chi(n) \cdot f(n x)
$$

for $f \in \mathscr{H}_{+}$. We now have the Euler product expansion

$$
\mathscr{L}_{\chi}=\sum_{n=1}^{\infty} \chi(n) \lambda_{n^{-1}}=\prod_{p \in \mathscr{P}} \sum_{e=0}^{\infty} \chi(p)^{e} \lambda_{p^{-e}}=\prod_{p \in \mathscr{P}}\left(1-\chi(p) \lambda_{p^{-1}}\right)^{-1} .
$$

The same estimates as for the Zeta operator show that this product expansion converges on $\mathscr{S}_{>}$(compare Proposition 3.2).

The Poisson Summation Formula looks somewhat different now: we have

$$
\mathscr{L}_{\chi}(f)=\kappa \cdot d^{1 / 2} \lambda_{d}^{-1} J \mathscr{L}_{\chi} \mathfrak{F}(f),
$$

where $\kappa$ is some complex number with $|\kappa|=1$. The proof of Theorem 3.3 then carries over without change. We also get the holomorphic continuation and the functional equation for $L_{\chi}$. If $\chi(-1)=-1$, we have to use the special function $2 x \exp \left(-\pi x^{2}\right)$ instead of $2 \exp \left(-\pi x^{2}\right)$ to pass from $L_{\chi}$ to the complete $L$-function. The results in Section 4 carry over in an evident way. Now $\mathscr{H}_{+}^{0}=$ $\{0\}$ because $L_{\chi}$ does not have poles, and the eigenvalues of ${ }^{t} D_{-}$are the nontrivial zeros of $L_{\chi}$, with correct algebraic multiplicity.

Some modifications are necessary in Section 5. We define $\iota_{-}$and $\pi_{-}$as above. Since we want the embeddings $\iota_{ \pm}$to agree on $\mathscr{H}_{\cap} \cong \mathscr{L}_{\chi} \mathscr{H}_{\cap}$, we should put

$$
\iota_{+}(f):=\left(\mathscr{L}_{\chi}(f), \kappa \cdot d^{1 / 2} \lambda_{d}^{-1} J \mathscr{L}_{\chi} \mathfrak{F} f\right)
$$

and modify $\pi_{+}$accordingly so that $\pi_{+} \iota_{+}=M_{\varphi}+\mathfrak{F}^{*} M_{\varphi} \mathfrak{F}$. With these changes, the remaining computations carry over easily. Of course, we get different local summands $W_{p}$ in the explicit formula for $\mathscr{L}_{\chi}$. You may want to compute them yourself as an exercise to test your understanding of the arguments above.

Even more generally, we can replace the rational numbers $\mathbb{Q}$ by an imaginary quadratic extension like $\mathbb{Q}[i]$ and study $L$-functions attached to characters of the idele class group of this field extension. Such fields have only one infinite place, which is complex. A character of the idele class group restricts to a character of the circle group inside $\mathbb{C}^{\times}$. The space $\mathscr{H}_{+}$is now replaced by the homogeneous subspace of $\mathscr{S}(\mathbb{C})$ defined by that character.

Once there is more than one infinite place, we need the more general setup of $[\mathbf{M e y}]$. In addition, the adelic constructions in [Mey] provide a better understanding even for $\mathbb{Q}$ because they show the similarity of the analysis at 
the finite and infinite places. The explicit formula takes a much nicer form if we put together all characters of the idele class group. The resulting local summands that make up the Weil distribution are of the same general form

$$
W_{v}(f)=p v \int_{\mathbb{Q}_{v}^{\times}} \frac{f(x)}{\left|1-x^{-1}\right|} d^{\times} x
$$

at all places $v\left(\right.$ with $\left.\mathbb{Q}_{\infty}=\mathbb{R}\right)$ and can also be interpreted geometrically as a generalised Lefschetz trace formula (see [Con99]).

\section{References}

[Con99] A. Connes - Trace formula in noncommutative geometry and the zeros of the Riemann zeta function, Selecta Math. (N.S.) 5 (1999), no. 1, p. 29-106.

[Gro55] A. GRothendiECK - Produits tensoriels topologiques et espaces nucléaires, Mem. Amer. Math. Soc. 1955 (1955), no. 16, p. 140.

[Mey] R. MEYER - On a representation of the idele class group related to primes and zeros of $L$-functions, Duke Math. J., to appear.

[Tat67] J. T. TATE - Fourier analysis in number fields, and Hecke's zeta-functions, in Algebraic Number Theory (Proc. Instructional Conf., Brighton, 1965), Thompson, Washington, D.C., 1967, p. 305-347. 

Mathematisches Institut, Seminars, (Y. TschINKel, ed.), p. 139-153

Universität Göttingen, 2004-05

\title{
REPRESENTATIONS OF FIELD AUTOMORPHISM GROUPS
}

\author{
M. Rovinsky \\ Independent University of Moscow, B. Vlasievsky Per. 11, 121002 Moscow, \\ Russia • E-mail : marat@mccme.ru
}

\begin{abstract}
We relate questions in birational algebraic geometry to representation theory.
\end{abstract}

In the first part we show how some geometric questions can be translated to the language of representation theory. There will be three categories of representations of $G: \mathscr{S}_{G} \supset \mathscr{I}_{G} \supset \mathscr{A} d m$, roughly corresponding to birational geometry, to birational motivic questions (like the structure of Chow groups of 0 -cycles) and to "finite-dimensional" birational motivic questions (in particular, description of "classical" motivic categories). This part is rather motivational, there will be many conjectures, few particular results, and almost no proofs.

In the second part it is explained in more detail, how appropriate representation theory could be developed by means of semi-linear representations. Main results suggest an explicit description of the category of admissible semi-linear representations, which is conjecturally sufficient for geometric applications.

Notation. Let $k$ be an algebraically closed field of characteristic zero, $F / k$ a universal domain, i.e., an algebraically closed extension of $k$ of countable transcendence degree, and $G=G_{F / k}=\operatorname{Aut}(F / k)$ the field automorphism group of $F$ over $k$ with the base of open subgroups $\left\{G_{F / k(x)}=\operatorname{Aut}(F / k(x)) \mid x \in F\right\}$.

November 18, 2004. 


\section{Translating geometric questions to the language of representation theory}

We are interested in representations of $G$. To specify the type of these representations, we have to ask a geometric question. There will be three categories of representations of $G$.

1.1. $\mathscr{S} m_{G}$. In general, geometry deals with varieties. To any variety $X$ over $k$ one can associate the $G$-module $\mathbb{Q}[X(F)]$, i.e. the $\mathbb{Q}$-vector space of 0-cycles on $X \times_{k} F$.

This representation is huge, but this is just a starting point.

Note that it is smooth, i.e. its stabilizers are open, so all representations we are going to consider will be smooth.

The first question to ask is: what are the finite-dimensional smooth representations of $G$ ?

Theorem 1.1 ([Rov05]). Any finite-dimensional smooth representation of $G$ is trivial.

This follows from the (topological) simplicity of $G$ :

Theorem 1.2 ([Rov05]). Any closed normal proper subgroup of $G$ is trivial.

\section{Remarks 1.3.}

1. One has $\mathbb{Q}[X(F)]=\bigoplus_{x \in X} \mathbb{Q}[\{k(x) \stackrel{/ k}{\hookrightarrow} F\}]$, so $\mathbb{Q}[X(F)]$ reflects rather the class of $X$ in the Grothendieck group $K_{0}\left(\operatorname{Var}_{k}\right)$ of partitions of varieties over $k$ than $X$ itself.

2. It is not clear, whether the birational type of $X$ is determined by the $G$-module $\mathbb{Q}[\{k(X) \stackrel{/ k}{\hookrightarrow} F\}]$ of generic 0 -cycles on $X$. E.g., if $X=Z \times \mathbb{P}^{1}$, $Y=Z^{\prime} \times \mathbb{P}^{1}$ and $Z^{\prime}$ is a twofold cover of $Z$ then there exist embeddings in both directions

$$
\mathbb{Q}[\{k(X) \stackrel{/ k}{\hookrightarrow} F\}] \hookrightarrow \mathbb{Q}[\{k(Y) \stackrel{/ k}{\hookrightarrow} F\}] \hookrightarrow \mathbb{Q}[\{k(X) \stackrel{/ k}{\hookrightarrow} F\}] .
$$

What is in common between $X$ and $Y$ is that their primitive motives coincide (and vanish).

However, we will see later how to extract "birational motivic" invariants "modulo isogenies", like $\operatorname{Alb}(X), \Gamma\left(X, \Omega_{X / k}^{\bullet}\right)$, out of $\mathbb{Q}[\{k(X) \stackrel{/ k}{\hookrightarrow} F\}]$. 
1.2. $\mathscr{A} d m$. Now consider a more concrete geometric category: the category of motives.

(Effective) pure covariant motives are pairs $(X, \pi)$ consisting of a smooth projective variety $X$ over $k$ with irreducible components $X_{j}$ and a projector $\pi=\pi^{2} \in \bigoplus_{j} B^{\operatorname{dim} X_{j}}\left(X_{j} \times_{k} X_{j}\right)$ in the algebra of correspondences on $X$ modulo numerical equivalence. The morphisms are defined by

$$
\operatorname{Hom}\left(\left(X^{\prime}, \pi^{\prime}\right),(X, \pi)\right)=\bigoplus_{i, j} \pi_{j} \cdot B^{\operatorname{dim} X_{j}}\left(X_{j} \times_{k} X_{i}^{\prime}\right) \cdot \pi_{i}^{\prime} .
$$

The category of pure covariant motives has an additive and a tensor structure: $\left(X^{\prime}, \pi^{\prime}\right) \bigoplus(X, \pi):=\left(X^{\prime} \coprod X, \pi^{\prime} \oplus \pi\right), \quad\left(X^{\prime}, \pi^{\prime}\right) \otimes(X, \pi):=\left(X^{\prime} \times_{k} X, \pi^{\prime} \times_{k} \pi\right)$.

A primitive $q$-motive is a pair $(X, \pi)$ with $\operatorname{dim} X=q$ and $\pi \cdot B^{q}\left(X \times_{k} Y \times \mathbb{P}^{1}\right)=0$ for any smooth projective variety $Y$ over $k$ with $\operatorname{dim} Y<q$. For instance, the category of the primitive 1-motives is equivalent to the category of abelian varieties over $k$ with morphisms tensored with $\mathbb{Q}$. It follows from a result of Jannsen [Jan92] that any pure motive is semi-simple and admits "primitive" decomposition $\bigoplus_{i, j} M_{i j} \otimes \mathbb{L}^{\otimes i}$, where $M_{i j}$ is a primitive $j$-motive and $\mathbb{L}=$ $\left(\mathbb{P}^{1}, \mathbb{P}^{1} \times\{0\}\right)$ is the Lefschetz motive.

Definition 1.4. A representation $W$ of a topological group is called admissible if it is smooth and the fixed subspaces $W^{U}$ are finite-dimensional for all open subgroups $U$.

Denote by $\mathscr{A} d m$ the category of admissible representations of $G$ over $\mathbb{Q}$.

Theorem 1.5 ([Rov05]). There is a fully faithful functor $\mathbb{B}^{\bullet}$ :

$\{$ pure covariant motives over $k\} \stackrel{\mathbb{B}^{\bullet}}{\longrightarrow}\left\{\begin{array}{c}\text { graded semi-simple admissible } \\ G \text {-modules of finite length }\end{array}\right\}$.

The grading corresponds to powers of the motive $\mathbb{L}$ in the "primitive" decomposition above.

Roughly speaking, the functor $\mathbb{B}^{\bullet}$ is defined by spaces of 0 -cycles defined over $F$ modulo "numerical equivalence over $k$ ". More precisely,

$$
\mathbb{B}^{\bullet}=\oplus_{j}^{\text {graded }} \lim _{L} \operatorname{Hom}\left([L]^{\text {prim }} \otimes \mathbb{L}^{\otimes j},-\right)
$$

is a graded direct sum of pro-representable functors. Here $L$ runs over all subfield of $F$ of finite type over $k$, and $[L]^{\text {prim }}$ is the quotient of the motive of any smooth projective model of $L$ over $k$ by all submotives of type $M \otimes \mathbb{L}$ for all effective motives $M$. 
Example 1.6. The motive of the point $\operatorname{Spec}(k)$ is sent to the trivial representation $\mathbb{Q}$ of degree 0 . The motive of a smooth proper curve $C$ over $k$ is sent to $\mathbb{Q} \oplus J_{C}(F) / J_{C}(F) \oplus \mathbb{Q}[1]$, where $J_{C}$ is the Jacobian of $C$ and $\mathbb{Q}[1]$ denotes the trivial representation in degree 1.

So, this inclusion is already a good reason to study admissible representations. Moreover, it is expected that

Conjecture 1.7. The functor $\mathbb{B}^{\bullet}$ is an equivalence of categories.

Of course, it would be more interesting to describe in a similar way the abelian category $\mathscr{M} \mathscr{M}$ of mixed motives over $k$, whose semi-simple objects are pure. This is one more reason to study the category $\mathscr{A} d m$ of admissible representations of $G$.

Theorem 1.8 ([Rov05]). The category $\mathscr{A} d m$ is abelian.

Proposition 1.9. For any $W \in \mathscr{A} d m$ and, conjecturally, for any effective motive $M$ one has

$$
\begin{array}{ll}
\operatorname{Ext}_{\mathscr{A} d m}^{>0}(\mathbb{Q}, W)=0 & \operatorname{Ext}_{\mathscr{M} \mathscr{M}}^{>0}(\mathbb{Q}, M)=0 \\
\operatorname{Ext}_{\mathscr{A} d m}^{1}\left(\frac{A(F)}{A(k)}, W\right)=\frac{\operatorname{Hom}_{\mathbb{Z}}\left(A(k), W W^{G}\right)}{\operatorname{Hom}_{G}\left(A(F) / A(k), W / W^{G}\right)} & \operatorname{Ext}_{\mathscr{M} \mathscr{M}}^{1}\left(H^{1}(A), M\right)=\frac{A(k) \otimes W_{0} M}{\operatorname{Hom}_{\mathscr{M}} \mathscr{M}^{(}\left(H^{1}(A), M / W_{0} M\right)} \\
\operatorname{Ext}_{\mathscr{A} d m}^{\geq 2}(A(F) / A(k), W)=0 & \operatorname{Ext}_{\mathscr{M} \mathscr{M}}^{\geq 2}\left(H^{1}(A), M\right)=0
\end{array}
$$

So we see that admissible representations of finite length should be related to effective motives. At least the Ext's between some irreducible objects are dual.

1.3. $\mathscr{I}_{G} \cdot$ The formal properties of $\mathscr{A} d m$ are not very nice. In particular, to prove Theorem 1.8 and Proposition 1.9 and to give an evidence to Conjecture 1.7 , one uses the inclusion of $\mathscr{A} d m$ to a bigger full subcategory in the category of smooth representations of $G$.

Definition 1.10. An object $W \in \mathscr{S} m_{G}$ is called "homotopy invariant" (in birational sense) if $W^{G / L}=W^{G / L^{\prime}}$ for any purely transcendental subextension $L^{\prime} / L$ in $F / k$. Denote by $\mathscr{I}_{G}$ the full subcategory in $\mathscr{S} m_{G}$ with "homotopy invariant" objects.

Remark 1.11. One can show that in this definition one can restrict oneself to $L^{\prime}$ 's of finite type over $k$, cf. [Rov05], $§ 6$. 
Example 1.12. For any smooth variety $X$ over $k$ one has $C H^{*}\left(X_{F}\right)_{\mathbb{Q}} \in$ $\mathscr{I}_{G}$, because of the descent property $C H^{*}\left(X_{F}\right)_{\mathbb{Q}}^{G_{F / L}}=C H^{*}\left(X_{L}\right)_{\mathbb{Q}}$.

\section{Theorem 1.13 ([Rov05]).}

1. The category $\mathscr{I}_{G}$ is a Serre subcategory in $\mathscr{S} m_{G}$.

2. $\mathscr{A} d m \subset \mathscr{I}_{G}$.

3. There exists a left adjoint functor $\mathscr{I}: \mathscr{S}_{m_{G}} \rightarrow \mathscr{I}_{G}$ to the inclusion $\mathscr{I}_{G} \hookrightarrow \mathscr{S} m_{G}$.

4. There are enough projectives in $\mathscr{I}_{G}$. Namely, the objects

$$
C_{k(X)}:=\mathscr{I} \mathbb{Q}[\{k(X) \stackrel{/ k}{\hookrightarrow} F\}]
$$

for all smooth irreducible varieties $X$ over $k$ form a system of projective generators of $\mathscr{I}_{G}$.

5. For any smooth proper variety $X$ over $k$ there is a canonical filtration $C_{k(X)} \supset \mathscr{F}^{1} \supset \mathscr{F}^{2} \supset \ldots$, canonical isomorphisms $C_{k(X)} / \mathscr{F}^{1}=\mathbb{Q}$ and $\mathscr{F}^{1} / \mathscr{F}^{2}=\operatorname{Alb}\left(X_{F}\right)_{\mathbb{Q}}$, and a non-canonical splitting

$$
C_{k(X)} \cong \mathbb{Q} \oplus \operatorname{Alb}\left(X_{F}\right)_{\mathbb{Q}} \oplus \mathscr{F}^{2} .
$$

The term $\mathscr{F}^{2}$ is determined by these conditions together with

$$
\operatorname{Hom}_{G}\left(\mathscr{F}^{2}, \mathbb{Q}\right)=\operatorname{Hom}_{G}\left(\mathscr{F}^{2}, A(F) / A(k)\right)=0
$$

for any abelian variety $A$ over $k$.

6. For any smooth proper variety $X$ over $k$ there is a canonical surjection $C_{k(X)} \rightarrow C H_{0}\left(X_{F}\right)_{\mathbb{Q}}$, which is an isomorphism for $X$ unirational over a curve (and in some other cases).

7. There exist (co-) limits in $\mathscr{I}_{G}$.

Proof of (2). Let $W \in \mathscr{A} d m, L$ an extension of $k$ in $F$ of finite type and $x, y \in$ $F$ are algebraically independent over $L$. Then the finite-dimensional space $W^{G_{F / L}}$ is included into the finite-dimensional spaces $W^{G_{F / L(x)}}$ and $W^{G_{F / L(y)}}$; and the latter ones are included into the finite-dimensional space $W^{\left.G_{F / L(x, y)}\right)}$. As the group $G_{F / L(x+y, x y)}$ is an extension of the group

$$
\{1, \alpha\}=\operatorname{Gal}(L(x, y) / L(x+y, x y))
$$

(so $\alpha x=y$ and $\alpha y=x$ ) by $G_{F / L(x, y)}$, one has $W^{G_{F / L(x+y, x y)}}=\left(W^{G_{F / L(x, y)}}\right)^{\langle\alpha\rangle}$. As the subgroups $G_{F / L(x+y, x y)}$ and $G_{F / L(x, y)}$ are conjugated in $G$, the spaces $W^{G_{F / L(x+y, x y)}}$ and $W^{G_{F / L(x, y)}}$ are of the same dimension. This implies that $W^{G_{F / L(x+y, x y)}}=W^{G_{F / L(x, y)}}$, and thus, $\alpha$ acts trivially on $W^{G_{F / L(x, y)}}$. 
Notice, however, that $\alpha$ permutes $W^{G_{F / L(x)}}$ and $W^{G_{F / L(y)}}$, so $W^{G_{F / L(x)}}=$ $W^{G_{F / L(y)}}$. One can show that the group generated by $G_{F / L(x)}$ and $G_{F / L(y)}$ is dense in $G_{F / L}$, and therefore, $W^{G_{F / L}}=W^{G_{F / L(x)}}$.

Together with the following two conjectures, this is a good reason to study $\mathscr{I}_{G}$.

Conjecture 1.14. For any smooth proper variety $X$ over $k$ the natural surjection $C_{k(X)} \longrightarrow C_{0}\left(X \times{ }_{k} F\right)_{\mathbb{Q}}$ is an isomorphism.

\section{Remarks 1.15.}

1. This, together with the motivic conjectures, implies that $\mathbb{B}^{\bullet}$ is an equivalence of categories (Conjecture 1.7).

2. Conjecture 1.14 implies that $\mathscr{I}_{G}$ admits the following commutative associative tensor structure: $W_{1} \otimes \mathscr{I} W_{2}:=\mathscr{I}\left(W_{1} \otimes W_{2}\right)$, and, in particular, $C_{k(X)} \otimes \mathscr{I} C_{k(Y)}=C_{k\left(X \times{ }_{k} Y\right)}$. $\Omega_{F / k}^{\bullet}$.

Conjecture 1.16. Any irreducible object of $\mathscr{I}_{G}$ is contained in the algebra

"Corollary" 1.17. Any irreducible object of $\mathscr{I}_{G}$ is admissible. So " $\mathscr{I}_{G} \approx$ $\mathscr{A} d m "$.

This conjecture 1.16 also has a geometric

"Corollary" 1.18. If $\Gamma\left(X, \Omega_{X / k}^{\geq 2}\right)=0$ for a smooth proper variety $X$ over $k$ then the Albanese map induces an isomorphism $C H_{0}(X)^{0} \stackrel{\sim}{\longrightarrow} \operatorname{Alb}(X)$. In that case $C_{k(X)}=C H_{0}\left(X_{F}\right)_{\mathbb{Q}}$.

This follows from the fact that $\operatorname{Hom}_{G}\left(C_{k(X)}, \Omega_{F / k}^{\bullet}\right)=\Gamma\left(X, \Omega_{X / k}^{\bullet}\right)$.

Conjecture 1.16 is one of the main motivations for what follows.

\section{From linear to semi-linear representations}

Look at the representation $\Omega_{F / k}^{\bullet}$. It carries an additional structure, of an $F$-vector space, so it is an example of semi-linear representation.

Definition 2.1. A semi-linear representation of $G$ over $F$ is an $F$-vector space $V$ endowed with an additive $G$-action $G \times V \rightarrow V$ such that $g(f v)=g f \cdot g v$ for any $g \in G, v \in V$ and $f \in F$. 
Denote by $\mathscr{C}$ the category of smooth semi-linear representations of $G$ over $F$.

Once again, we are interested in linear representations of $G$, especially in irreducible ones, and more particularly, in irreducible "homotopy invariant" representations, i.e. objects of $\mathscr{I}_{G}$. But how to study (the irreducible objects of) $\mathscr{S} m_{G}$ using $\mathscr{C}$, and why $\mathscr{C}$ ?

One has the faithful functor $\mathscr{C} \stackrel{\text { for }}{\longrightarrow} \mathscr{S} m_{G}$ admitting a left adjoint functor $\mathscr{S} m_{G} \stackrel{\otimes F}{\longrightarrow} \mathscr{C}$, so $W \hookrightarrow$ for $(W \otimes F)$.

Of cource, $W \otimes F$ can be reducible, even if $W$ is irreducible, but there is an irreducible semi-linear quotient $V$ of $W \otimes F$ with an inclusion $W \subset V$, so any irreducible object of $\mathscr{S} m_{G}$ is contained in an irreducible object of $\mathscr{C}$.

It is well-known (Satz 90) that any smooth semi-linear Galois representation is trivial.

This gives a hint that, dispite the existence of non-trivial smooth semi-linear representations of $G$ over $F$, it is easier to study them than the $(\mathbb{Q}$-)linear representations of $G$.

Example 2.2 (of simplification). Let $A$ be an abelian variety over $k$. Any sufficiently general 1 -form $\eta \in \Gamma\left(A, \Omega_{A / k}^{1}\right)$ gives an embedding $A(F) / A(k) \hookrightarrow \Omega_{F / k}^{1}$ by sending the point $k(A) \stackrel{\sigma}{\rightarrow} F$ to $\sigma \eta \in \Omega_{F / k}^{1}$.

Thus, all representations of $G$ corresponding to pure 1-motives are contained in the irreducible object $\Omega_{F / k}^{1}$ of $\mathscr{C}$.

Now, we begin the study of smooth semi-linear representations of $G$.

Theorem 2.3 ([Rov05]). Any finite-dimensional smooth semi-linear representation of $G$ over $F$ is trivial.

(In fact, any smooth $G$-torsor under the smooth $G$-group $B(F)$ is trivial for any algebraic $k$-group $B$, cf. [Rov05]. The principal part of the proof is to show that there is a representative with values in $B(k)$ of the class of any smooth torsor in $H^{1}(G, B(F))$. And then one applies the simplicity of $G$.

A natural extension of the notion of finite-dimensional semi-linear representation is the notion of admissible semi-linear representation.

Definition 2.4. A smooth semi-linear representation $V$ of $G$ over $F$ is called admissible if, for any open subgroup $U \subseteq G$, the fixed subspace $V^{U}$ is finitedimensional over the fixed subfield $F^{U}$. 
Proposition 2.5. The admissible semi-linear representations of $G$ over $F$ form an additive tensor category, denoted by $\mathscr{A}$.

This follows from the formula $\left(V_{1} \otimes_{F} V_{2}\right)^{G_{F / L}}=V_{1}^{G_{F / L}} \otimes_{L} V_{2}^{G_{F / L}}$ for any subfield $k \subseteq L=\bar{L} \subseteq F$ and any $V_{1}, V_{2} \in \mathscr{C}$, cf. [Rov], which is again based on the simplicity of $G$. 0 .

Example 2.6. The objects $\Omega_{F / k}^{\bullet}$ and $\bigotimes_{F}^{q} \Omega_{F / k}^{1}$ are admissible for any $q \geq$

The rest of these notes is concerned with the category $\mathscr{A}$. The questions are: what one could expect of this category, and what are the reasons for that?

We shall see in Theorem 4.5 that the category $\mathscr{A}$ is abelian, and $F$ is its projective object (this is Proposition 4.4). There will be explained some evidences for the following

\section{Conjecture 2.7.}

1. The functors $\operatorname{Hom}_{\mathscr{C}}\left(\otimes_{F}^{q} \Omega_{F / \mathbb{Q}}^{1},-\right)$ are exact on $\mathscr{A}$ for any $q \geq 0$.

2. Irreducible objects of $\mathscr{A}$ are direct summands of the tensor algebra $\bigotimes_{F}^{\bullet} \Omega_{F / k}^{1}$.

It follows from this conjecture that if $V, V^{\prime}$ are irreducible objects of $\mathscr{A}$ and $\operatorname{Ext}_{\mathscr{A}}^{j}\left(V, V^{\prime}\right) \neq 0$ then there is a surjection $V^{\prime} \otimes_{F} \otimes_{F}^{j} \Omega_{F / k}^{1} \rightarrow V$ and $\operatorname{Ext}_{\mathscr{A}}^{j}\left(\Omega_{F / k}^{j+q}, \Omega_{F / k}^{q}\right)=\operatorname{Hom}_{k}\left(\operatorname{Sym}_{k}^{j} \Omega_{k}^{1}, k\right)$. If $k=\overline{\mathbb{Q}}$ is the field of algebraic numbers then $\mathscr{A}$ is semi-simple.

The isomorphism $\operatorname{Ext}_{\mathscr{A}}^{1}\left(\Omega_{F / k}^{1}, F\right)=\operatorname{Der}(k)$ associates to any non-zero derivation $\eta: \Omega_{k}^{1} \rightarrow k$ the class of extension

$$
0 \rightarrow F \stackrel{\cdot \eta^{-1}(1)}{\longrightarrow} \Omega_{F}^{1} / \operatorname{ker} \eta \otimes_{k} F \rightarrow \Omega_{F / k}^{1} \rightarrow 0 .
$$

\section{Remarks 2.8.}

1. There exist smooth irreducible semi-linear representations that are not in $\bigotimes_{F}^{\bullet} \Omega_{F / k}^{1}$. For instance, anyone containing an irreducible quotient of the cyclic module $\mathbb{Q}[\overline{k(x)} \mid x \in F-k]^{\circ}$ of formal linear combinations over $\mathbb{Q}$ of degree zero of algebraically closed subfield in $F$ of transcendene degree 1 over $k$. So one cannot replace the category $\mathscr{A}$ in the part 2 of Conjecture 2.7 by the whole category $\mathscr{C}$. 
2. Assuming the part 2 of Conjecture 2.7, one can reformulate Conjecture 1.16 in the following linguistically more convencing form:

Any irreducible object of $\mathscr{A} d m$ is contained in an irreducible object of $\mathscr{A}$.

This reformulation is based on the following fact.

Proposition 2.9 ([Rov $]$ ). Any G-morphism from an object of $\mathscr{I}_{G}$ to tensor algebra $\bigotimes_{F}^{\bullet} \Omega_{F / k}^{1}$ factors through the exterior algebra $\Omega_{F / k}^{\bullet}$.

\section{Sketch of the proofs and the source of Conjecture 2.7}

Here I start explaining the source of Conjecture 2.7. Fix a transcendence basis $x_{1}, x_{2}, x_{3}, \ldots$ of $F$ over $k$. Set $K_{n}=k\left(x_{1}, \ldots, x_{n}\right)$. Consider the towers

$$
\begin{aligned}
& \mathbb{A}_{k}^{1} \leftarrow \mathbb{A}_{k}^{2} \leftarrow \mathbb{A}_{k}^{3} \leftarrow \cdots \\
& \bigcup_{Y_{1}} \leftarrow Y_{2} \leftarrow \bigcup_{3} \leftarrow \ldots
\end{aligned}
$$

where $Y_{n}:=\operatorname{Spec}\left(k\left[x_{1}^{ \pm 1}, \ldots, x_{n}^{ \pm 1}\right]\right) \subset \mathbb{A}_{k}^{n}:=\operatorname{Spec}\left(k\left[x_{1}, \ldots, x_{n}\right]\right)$. Let $\mathbb{P}_{k}^{n} \supset \mathbb{A}_{k}^{n}$ be the natural compactification with respect to the affine coordinates $x_{1}, \ldots, x_{n}$.

Any object of $\mathscr{A}$ induces a finite-dimensional semi-linear representation $V_{n}:=V^{G_{F / K_{n}}}$ of the group $G_{n}:=\operatorname{Aut}\left(\mathbb{P}_{k}^{n} / k\right) \cong \operatorname{PGL}_{n+1} k$ over $K_{n}$. Our goal is to find out exactly, which one.

In fact, $V_{n}$ is a (non-degenerate) semi-linear representation of the semi-group of dominant rational self-maps of $\mathbb{P}_{k}^{n}$. In particular, of

$$
\operatorname{End}_{\text {dom }}\left(Y_{n} / k\right) \cong \operatorname{Mat}_{n \times n}^{\operatorname{det} \neq 0} \mathbb{Z} \ltimes T_{n},
$$

where $T_{n} \subset G_{n}$ is the maximal torus acting freely on $Y_{n}$, acts on $V_{n}$. "Nondegenerate" means that the action of any element of the semi-group is injective.

Proposition 3.1 ([Rov $]$ ). Any finite-dimensional semi-linear representation $V_{n}$ of the semi-group $\operatorname{End}_{\mathrm{dom}}\left(Y_{n} / k\right)$ over $K_{n}$ is induced by a k-linear representation.

Let, for each integer $\ell \neq 0$ the element $\sigma_{\ell} \in \operatorname{End}_{\mathrm{dom}}\left(Y_{n} / k\right)$ raises all $x_{j}$ 's to the $\ell$-th power. Then for any $\tau \in T_{n}$ one has $\sigma_{\ell} \tau^{\ell}=\tau \sigma_{\ell}$. From this we get

Corollary 3.2. The restriction of $V_{n}$ to $T_{n}$ is induced by a unipotent representation $V_{n}^{T_{n}^{\text {tors }}}$. 
Proof. One has $\sigma_{\ell} \tau^{\ell}=\tau \sigma_{\ell}$, where $\sigma_{\ell} x_{j}=x_{j}^{\ell}$ for any $1 \leq j \leq n$ and $\tau \in T_{n}$. Varying integer $\ell \geq 2$, we see that for any representation

$$
\rho: \operatorname{End}_{\mathrm{dom}}\left(Y_{n} / k\right) \rightarrow \mathrm{GL}_{N} k
$$

the element $\rho(\tau) \in \mathrm{GL}_{N} k$ is conjugated to its arbitrary power, so $\rho(\tau)$ is unipotent.

Scheme of the proof of the proposition. This is a game with cocycles with the goal to make them constant.

1. Reduction to the case $n=1$ (and any algebraically closed $k$ of characteristic zero).

2. Reduction to the local claim: $H^{1}\left(\mathbb{N}, \mathrm{GL}_{N} k\right) \stackrel{\sim}{\longrightarrow} H^{1}\left(\mathbb{N}, \mathrm{GL}_{N} k((t))\right)$.

Let $\left(f_{\sigma}\right) \in Z^{1}\left(\mathbb{Z}_{\neq 0} \ltimes k^{\times}, \mathrm{GL}_{N} k(t)\right)$. We embed $k(t)$ into $k((t))$. Assuming that $f_{\xi}$ is regular at $0: f_{\xi} \in \mathrm{GL}_{N} k[[t]]$, where $\xi t=t^{\ell}$ for some $\ell \geq 2$, there is an explicit (cf. below) $\Phi \in \mathrm{GL}_{N} k[[t]]$ such that $\Phi \equiv 1 \bmod t$ and $\Phi \cdot f_{\xi} \cdot \xi \Phi^{-1}=$ $f_{\xi}(0) \in \mathrm{GL}_{N} k$.

Using functional equations (the cocycle condition) and assuming that $k$ is the field of complex numbers, one shows that $\Phi$ is meromorphic on $\mathbb{P}^{1}$, i.e. rational. We may, thus, assume that $f_{\xi} \in \mathrm{GL}_{N} k$. Commutation relations in $\mathbb{Z}_{\neq 0} \ltimes k^{\times}$imply that $f_{\sigma} \in \mathrm{GL}_{N} k$ for any $\sigma$.

3. Proof of the local claim. The first step:

$$
H^{1}\left(S, \mathrm{GL}_{N} k\right) \stackrel{\sim}{\longrightarrow} H^{1}\left(S, \mathrm{GL}_{N} k[[t]]\right)
$$

for any sub-semigroup $S \subseteq \mathbb{N}$.

Proof. Let $S \neq\{1\}, p \in S-\{1\}$ and $\left(f_{\ell}\right) \in Z^{1}\left(S, \mathrm{GL}_{N} k[[t]]\right)$. Set $\Phi=$ $\lim _{s \rightarrow \infty} f_{p^{s}}(0) f_{p^{s}}(t)^{-1}$. This is the same element of $1+t \mathfrak{g l}_{N} k[[t]]$ as in 2 . Then $\Phi(t)$. $f_{p}(t) \cdot \Phi\left(t^{p}\right)^{-1}=f_{p}(0)$, so we may suppose that $f_{p} \in \mathrm{GL}_{N} k$. As $f_{p}^{-1} f_{\ell}(t) f_{p}=$ $f_{\ell}\left(t^{p}\right)$, we get $f_{\ell} \in \mathrm{GL}_{N} k$ for any $\ell \in S$.

Thus, we get a functor $\mathscr{A} \rightarrow \mathfrak{S} \mathfrak{L}_{n}^{u}$, where $\mathfrak{S}_{\mathfrak{L}_{n}^{u}}^{u}$ is the category of finitedimensional $K_{n}$-semi-linear $G_{n}$-representations whose restrictions to the maximal torus $T_{n}$ in $G_{n}$ are induced by unipotent representations. We shall see that $\mathscr{A}$ is abelian and that this functor is exact.

Clearly, $\mathfrak{S}_{\mathfrak{L}_{n}^{u}}^{u}$ is an abelian neutral tannakian category with the fibre functor $H^{0}\left(T_{n}^{\mathrm{tors}},-\right): \mathfrak{S}_{\mathfrak{L}_{n}^{u}} \rightarrow \mathscr{V} e c_{k}$.

Theorem 3.3 ([Rov $]$ ). For any integer $n \geq 2$ there exists a fully faithful functor

$$
\mathfrak{S}_{\mathfrak{L}_{n}^{u}}^{u} \stackrel{\mathscr{S}}{\rightarrow}\left\{\text { coherent } G_{n} \text {-sheaves on } \mathbb{P}_{k}^{n}\right\}
$$


The composition of $\mathscr{S}$ with the generic fibre functor is the identical full embedding of $\mathfrak{S}_{\mathfrak{L}_{n}^{u}}^{u}$ to the category of finite-dimensional $K_{n}$-semi-linear $G_{n}$-representations.

(One says that $\mathscr{V}$ is a $G_{n}$-sheaf if it is endowed with a collection of isomorphisms $\alpha_{g}: \mathscr{V} \stackrel{\sim}{\longrightarrow} g^{*} \mathscr{V}$ for each $g \in G_{n}$ satisfying the chain rule: $\alpha_{h g}=$ $g^{*} \alpha_{h} \circ \alpha_{g}$ for any $g, h \in G_{n}$.)

Proof. Consists of checking that $\left.\mathscr{S}(V)\right|_{Y_{n}}:=H^{0}\left(T_{n}^{\text {tors }}, V\right) \otimes_{k} \mathscr{O}_{Y_{n}} \subset V$ happily glue together, when $Y_{n}$ varies. The main step is to show that

$$
H^{0}\left(U_{0},-\right): \mathfrak{S} \mathfrak{L}_{n}^{u} \rightarrow \mathscr{V} e c_{k}
$$

is also a fibre functor, where $U_{0}$ is a lattice over a cyclotomic subfield of $k$ in the unipotent radical of the stabilizer of a hyperplane in $\mathbb{P}_{k}^{n}$. (This fibre functor will be used in the proof of Lemma 4.1.)

Proposition 3.4. If $n \geq 2$ then any irreducible object of $\mathfrak{S}_{n}^{u}$ is the generic fibre of an irreducible coherent $G_{n}$-equivariant sheaf on $\mathbb{P}_{k}^{n}$, i.e., a direct summand of the semi-linear representation $\operatorname{Hom}_{K_{n}}\left(\left(\Omega_{K_{n} / k}^{n}\right)^{\otimes M}, \bigotimes_{K_{n}}^{\bullet} \Omega_{K_{n} / k}^{1}\right)$ for an appropriate $M$.

Proof. We have to check that the $T_{n}$-action on $V^{T_{n}^{\text {tors }}}=\Gamma\left(Y_{n}, \mathscr{V}\right)^{T_{\text {tors }}}$ is rational, i.e. trivial (since it is unipotent) for any irreducible object $V$ of $\mathfrak{S}_{\mathfrak{L}_{n}^{u}}^{u}$. As $G_{n}$ is generated by a finite number of conjugates of $T_{n}$, this will imply that $\mathscr{V}$ is equivariant, i.e., the action $G_{n} \times \operatorname{tot}(\mathscr{V}) \rightarrow \operatorname{tot}(\mathscr{V})$ is a morphism of $k$-varieties.

Let $E$ be the total space of the vector bundle on $\mathbb{P}_{k}^{n}$ with the sheaf of sections $\mathscr{S}(V)$. The $G_{n}$-structure on $E$ is a homomorphism $\tau: G_{n} \rightarrow \operatorname{Aut}_{\text {lin }}(E)$ splitting the projection $\operatorname{Aut}_{\text {lin }}(E) \rightarrow G_{n}$. Here $\operatorname{Aut}_{\text {lin }}(E)$ is the group of automorphisms of the $k$-variety $E$ over $k$ inducing linear transforms between the fibres. It is not hard to deduce from the irreducibility of $V$ that the Zariski closure $\overline{\tau\left(G_{n}\right)}$ of $\tau\left(G_{n}\right)$ is reductive.

For a commutative finite $k$-algebra $A$ denote by $R_{A / k}$ the Weil functor of restriction of scalars on $A$-schemes, cf. [DG70], I, §1, 6.6. To proceed futher in the proof, we need the following theorem of Borel-Tits.

Theorem 3.5 ([BT73], Théorème 8.16). Let $G$ be a simply connected absolutely almost simple $k$-group, and $G^{\prime}$ be a reductive $k$-group, $\tau: G(k) \rightarrow$ $G^{\prime}(k)$ a homomorphism with Zariski dense image. Let $G_{1}^{\prime}, \ldots, G_{m}^{\prime}$ be the almost simple normal subgroups of $G^{\prime}$.

Then there exist field embeddings $\varphi_{i}: k \rightarrow k$, an isogeny $\beta: \prod_{i=1}^{m}{ }^{\varphi_{i}} G \rightarrow G^{\prime}$ (here ${ }^{\varphi_{i}} G:=G \times_{k, \varphi_{i}} k$ ) and a homomorphism $\mu: G(k) \rightarrow Z_{G^{\prime}}(k)$ such that 
$\beta\left({ }^{\varphi_{i}} G\right)=G_{i}^{\prime}$ and $\tau(h)=\mu(h) \cdot \beta\left(\prod_{i=1}^{m} \varphi_{i}^{\circ}(h)\right)$ for any $h \in G(k)$ (here $\varphi_{i}^{\circ}$ : $G(k) \rightarrow{ }^{\varphi_{i}} G(k)$ is the canonical homomorphism).

Taking $G=\mathrm{SL}_{n+1} k$ and $G^{\prime}=\operatorname{Aut}_{\text {lin }}(E)$ in Theorem 3.5, we get

Corollary 3.6. In the above notation the Zariski closure of $\tau(T(k))$ is a torus in $\operatorname{Aut}_{\text {lin }}(E)$ for any torus $T \subset G_{n}$.

Any rational representation of a torus is semi-simple, which implies that $V^{T_{n}^{\text {tors }}}$ is a trivial representation of $T_{n}$, and thus, completes the proof of Proposition 3.4, since the generic fibres of irreducible $G_{n}$-equivariant sheaves on $\mathbb{P}_{k}^{n}$ are exactly of the desired type.

Theorem 3.7 ([Rov04]). For any irreducible F-semi-linear representation

$F \nRightarrow V \in \mathscr{A}$ any irreducible subquotient of the $K_{n}$-semi-linear $G_{n}$ representation $V^{G_{F / K_{n}}}$ is a direct summand of $\bigotimes_{K_{n}}^{\geq 1} \Omega_{K_{n} / k}^{1}$.

This is an evidence for Conjecture 2.7 part 2.

Proof. Keeping in mind Proposition 3.4 we have only to eliminate $K_{n}$ and the "negative twists" by powers of $\Omega_{K_{n} / k}^{n} . K_{n}$ will be eliminated later in Proposition 4.4. One has $V^{G_{F / K_{n}}} \subseteq\left(V^{G_{F / K_{n+1}}}\right)^{H}$, where $H=G_{n+1} \cap G_{K_{n+1} / K_{n}}$, so it suffices to show that $\left(S^{\lambda} \Omega_{K_{n+1} / k}^{1} \otimes\left(\Omega_{K_{n+1} / k}^{n+1}\right)^{\otimes(-s)}\right)^{H}=0$ for a Young diagram $\lambda$ with no columns of height $n+1$ and some integer $s>0$.

The latter follows from

Lemma 3.8. Let $W$ be an $(n+1)$-dimensional $k$-vector space, $L \subset W$ be a one-dimensional subspace,

$$
H_{\text {lin }}=\operatorname{ker}[\mathrm{GL}(W, L) \rightarrow \mathrm{GL}(W / L)] \cong k^{\times} \ltimes \operatorname{Hom}(W / L, L) .
$$

For any Young diagram $\lambda$ with no columns of height $n+1$ one has

$$
\left(S^{\lambda} W^{\vee} \otimes_{k}(\operatorname{det} W)^{\otimes s}\right)^{H_{\text {lin }}}= \begin{cases}S^{\lambda}(W / L)^{\vee} & \text { if } s=0, \\ 0 & \text { otherwise }\end{cases}
$$

\section{Extensions in $\mathfrak{S} \mathfrak{L}_{n}^{u}$ and in $\mathscr{A}$}

Denote by $\operatorname{Aff}_{n}^{(\ell)} \mathbb{Q}$ the subgroup of $G_{n}$ consisting of the $\mathbb{Q}\left(\mu_{\ell}\right)$-affine substitutions of $x_{1}, \ldots, x_{n}$ with Jacobian in the group $\mu_{\ell}$ of $\ell$-th roots of unity in $k$, and by $\operatorname{SAff}_{n}^{(\ell)} \mathbb{Q}$ the evident subgroup of index $\ell$. 
Lemma 4.1. Let $n \geq 2$ and $\ell \geq 2$. Then restriction of any object $V \in \mathfrak{S} \mathfrak{L}_{n}^{u}$ to $\mathrm{SAff}_{n}^{(\ell)} \mathbb{Q}$ is induced by a rational representation of its reductive quotient $\mathrm{SL}_{n} \mathbb{Q}\left(\mu_{\ell}\right)$ over $k$. Irreducible rational representations of $\mathrm{SL}_{n} \mathbb{Q}\left(\mu_{\ell}\right)$ over $k$ induce irreducible semi-linear representations of $\mathrm{SAff}_{n}^{(\ell)} \mathbb{Q}$ over $K_{n}$. In particular, any extension in $\mathfrak{S}_{\mathfrak{L}_{n}^{u}}^{u}$ splits as an extension of $K_{n}$-semi-linear representations of $\operatorname{SAff}_{n}^{(\ell)} \mathbb{Q}$.

Proof. It is shown in [Rov] that $H^{0}\left(U_{0},-\right)$ is the fibre functor on $\mathfrak{S}_{\mathfrak{L}_{n}}^{u}$, where $U_{0}$ is the unipotent radical of $\operatorname{SAff}_{n}^{(\ell)} \mathbb{Q}$, so $V=V^{U_{0}} \otimes_{k} K_{n}$ and the restriction of $V$ to $\mathrm{SAff}_{n}^{(\ell)} \mathbb{Q}$ is induced by a $k$-linear representation $V^{U_{0}}$ of $\mathrm{SL}_{n} \mathbb{Q}\left(\mu_{\ell}\right)$ for any $V \in \mathfrak{S} \mathfrak{L}_{n}^{u}$.

By Proposition 3.4, the irreducible subquotients of $V$ restricted to $\operatorname{SAff}_{n}^{(\ell)} \mathbb{Q}$ are induced by rational irreducible representations of $\operatorname{SL}_{n} \mathbb{Q}\left(\mu_{\ell}\right)$, so the irreducible subquotients of $V^{U_{0}}$ are rational and induce irreducible semi-linear representations of $\operatorname{SAff}_{n}^{(\ell)} \mathbb{Q}$ over $K_{n}$.

It follows from Theorems 3.5 and 4.7 that the $k$-linear representation $V^{U_{0}}$ of $\mathrm{SL}_{n} \mathbb{Q}\left(\mu_{\ell}\right)$ is semi-simple.

Lemma 4.2. Let $n, \ell \geq 2$ and $s$ be some integers such that $\ell$ does not divide $s+1$. Let $V=S_{K_{n}}^{\lambda} \Omega_{K_{n} / k}^{1} \otimes_{K_{n}}\left(\Omega_{K_{n} / k}^{n}\right)^{\otimes s}$ for a Young diagram $\lambda$ with columns of height $<n$.

Then $\left(V^{\mathscr{H}_{n}^{(\ell)}}\right)^{\mathrm{Aff}_{n-1}^{(\ell)} \mathbb{Q}}=V^{\mathrm{Aff}}{ }_{n}^{(\ell)} \mathbb{Q}$, where $\mathscr{H}_{n}^{(\ell)}:=G_{K_{n} / K_{n-1}} \cap \operatorname{Aff}_{n}^{(\ell)} \mathbb{Q}$.

Proof. Consists of a direct computation using Lemma 3.8.

Lemma 4.3 ([Rov], Lemma 7.1). Let $n>m \geq 0$ be integers and $H$ be a subgroup of $G_{F / k}$ preserving $K_{n}$ and projecting onto a subgroup of $G_{K_{n} / k}$ containing the permutation group of the set $\left\{x_{1}, \ldots, x_{n}\right\}$. Then the subgroup in $G_{F / k}$ generated by $G_{F / K_{m}}$ and $H$ is dense.

For any $U \in \mathscr{A}$ and $m \geq 0$ set $U_{m}=U^{G_{F / K_{m}}}$.

Proposition 4.4. If $U \in \mathscr{A}$ and there is a subquotient of $U_{n} \in \mathfrak{S}_{n}^{u}$ isomorphic to $K_{n}$ then there is an embedding $F \hookrightarrow V$ in $\mathscr{A}$. One has

$$
H_{\text {smooth }}^{1}\left(G_{F / k}, V\right)=0, \text { for any } V \in \mathscr{A} \text {. }
$$

Proof. By Lemma 4.3, $U^{G_{F / k}}=U_{n+1}^{\mathrm{Aff}_{n+1}^{(\ell)} \mathbb{Q}} \cap U_{n}$ for any even $\ell \geq 2$. By Theorem 3.7, Lemma 4.1 and Lemma 4.2, $\left(U_{n+1}^{\mathscr{H}_{n+1}^{(\ell)}}\right)^{\mathrm{Aff}_{n}^{(\ell)} \mathbb{Q}}=U_{n+1}^{\mathrm{Aff}_{n+1}^{(\ell)} \mathbb{Q}}$ for any sufficiently big $\ell$, so, as $U_{n} \subseteq U_{n+1}^{\mathscr{H}_{n+1}^{(\ell)}}$, one has $U^{G_{F / k}}=U_{n}^{\mathrm{Aff}_{n}^{(\ell)} \mathbb{Q}}$, and thus, $U^{G_{F / k}} \neq 0$ if there is a subquotient of $U_{n} \in \mathfrak{S}^{u} \mathfrak{L}_{n}^{u}$ isomorphic to $K_{n}$. 
Clearly, $H^{0}(G,-)=\operatorname{Hom}_{G}(\mathbb{Q},-)=\operatorname{Hom}_{\mathscr{C}}(F,-)$ and

$$
H_{\text {smooth }}^{1}(G,-)=\operatorname{Ext}_{\mathscr{S} m_{G}}^{1}(\mathbb{Q},-)=\operatorname{Ext}_{\mathscr{C}}^{1}(F,-)
$$

on $\mathscr{C}$, so we have to show that any smooth $F$-semi-linear extension

$$
0 \rightarrow V \rightarrow U \rightarrow F \rightarrow 0
$$

splits. Fix $n>1$ such that $U_{n}$ surjects onto $K_{n}$. By Theorem 3.7 and Lemma 4.1 the semi-linear representation $U_{n}$ of $\operatorname{Aff}_{n}^{(\ell)} \mathbb{Q}$ over $K_{n}$ splits as $K_{n} \oplus V_{n}$, and thus, $U^{G_{F / k}}$ projects onto $k$.

Theorem 4.5. The category $\mathscr{A}$ is abelian.

Proof. Let $V \in \mathscr{A}$ and $V \stackrel{\pi}{\rightarrow} V^{\prime}$ be a surjection of $F$-semi-linear representations of $G_{F / k}$. By Proposition 4.4, for any $K \subset F$ of finite type over $k$ and any $v \in\left(V^{\prime}\right)^{G_{F / \bar{K}}}-\{0\}$, the extension

$$
0 \rightarrow \operatorname{ker} \pi \rightarrow \pi^{-1}(F \cdot v) \rightarrow F \rightarrow 0
$$

of $F$-semi-linear representations of $G_{F / \bar{K}}$ splits. This implies that the natural projection $V^{G_{F / K}} \stackrel{\pi_{K}}{\longrightarrow}\left(V^{\prime}\right)^{G_{F / K}}$ is surjective, and thus, $V^{\prime}$ is also an admissible semi-linear representation.

4.1. Extensions in $\mathfrak{S}_{\mathfrak{L}_{n}^{u}}^{u}$. Now we need the following particular case of Bott's theorem.

Theorem 4.6 (Bott). If $\mathscr{V}$ is an irreducible $G_{n}$-equivariant coherent sheaf on $\mathbb{P}_{k}^{n}$ then there exists at most one $j \geq 0$ such that $H^{j}\left(\mathbb{P}_{k}^{n}, \mathscr{V}\right) \neq 0$. If $H^{j}\left(\mathbb{P}_{k}^{n}, \mathscr{V}\right)^{G_{n}} \neq 0$ then $\mathscr{V} \cong \Omega_{\mathbb{P}_{k}^{n} / k}^{j}$.

And the following elaboration of Remark 8.19 of [BT73] and [Tit74], §5.1:

Theorem 4.7 ([LR01], Theorem 3). Let $G$ be a simple simply connected Chevalley $k$-group, $\mathscr{G}$ a connected algebraic $k$-group and $\tau: G(k) \rightarrow \mathscr{G}(k)$ a homomorphism with Zariski dense image. Assume that the unipotent radical $\mathscr{G}_{u}$ of $\mathscr{G}$ is commutative and the composition $G(k) \stackrel{\tau}{\rightarrow} \mathscr{G}(k) \rightarrow G^{\prime}(k)$, where $G^{\prime}=\mathscr{G} / \mathscr{G}_{u}$, is induced by a rational k-morphism $\lambda: G \rightarrow G^{\prime}$. Let $A=$ $k\left[\varepsilon_{1}, \ldots, \varepsilon_{r}\right] /\left(\varepsilon_{1}^{2}, \ldots, \varepsilon_{r}^{2}\right)$ and $\mathscr{H}=R_{A / k}\left(G \times_{k} A\right) \cong G \ltimes \mathfrak{g}^{\oplus r}$, where $\mathfrak{g}=\operatorname{Lie}(G)$ is the adjoint representation of $G$, and $r=\operatorname{dim} \mathscr{G}_{u} / \operatorname{dim} G^{\prime}$.

Then $\mathscr{G}_{u}$ splits into a direct sum of $r$ copies of the adjoint representation of $G^{\prime}$; and there exist derivations $\delta_{1}, \ldots, \delta_{r}$ of $k$ and a $k$-isogeny $\mu: \mathscr{H} \rightarrow \mathscr{G}$ such that $\tau=\mu \circ \eta_{\delta}$, where $\eta_{\delta}: G(k) \rightarrow \mathscr{H}(k)$ is induced by the ring embedding $i d+\sum_{j=1}^{r} \delta_{j} \varepsilon_{j}: k \rightarrow A$. 
Using these theorems and the functor $\mathscr{S}$ of Theorem 3.3, it is not hard to deduce by techniques similar to that of the proof of Proposition 3.4 the following

Proposition 4.8 ([Rov04]). Let $n \geq 2$. Suppose that $\operatorname{Ext}_{\mathfrak{S}^{u}{ }_{n}^{u}}^{1}\left(K_{n}, V_{\circ}\right) \neq 0$ for some irreducible object $V_{\circ}$ of $\mathfrak{S}_{\mathfrak{L}_{n}^{u}}^{u}$. Then either $V_{\circ} \cong \Omega_{K_{n} / k}^{1}$, or $V_{\circ} \cong$ $\operatorname{Der}\left(K_{n} / k\right)$. One has

$$
\operatorname{Ext}_{\mathfrak{S} \mathfrak{L}_{n}^{u}}^{1}\left(K_{n}, \Omega_{K_{n} / k}^{1}\right)=k \text { and } \operatorname{Ext}_{\mathfrak{S} \mathfrak{L}_{n}^{u}}^{1}\left(K_{n}, \operatorname{Der}\left(K_{n} / k\right)\right)=\operatorname{Der}(k) .
$$

\section{References}

[BT73] A. Borel \& J. Tits - Homomorphismes "abstraits" de groupes algébriques simples, Ann. of Math. (2) 97 (1973), p. 499-571.

[DG70] M. Demazure \& P. Gabriel - Groupes algébriques. Tome I: Géométrie algébrique, généralités, groupes commutatifs, Masson \& Cie, Éditeur, Paris, 1970, Avec un appendice Corps de classes local par Michiel Hazewinkel.

[Jan92] U. JANnsEn - Motives, numerical equivalence, and semi-simplicity, Invent. Math. 107 (1992), no. 3, p. 447-452.

[LR01] L. LIFSCHITZ \& A. RAPINCHUK - On abstract homomorphisms of Chevalley groups with nonreductive image. I, J. Algebra 242 (2001), no. 1, p. 374-399.

[Rov] M. Rovinsky - Semi-linear representations of PGL, math.RT/0306333.

[Rov04] _ Admissible semi-linear representations, 2004, Preprint MPIM2004122, Bonn.

[Rov05] , Motives and admissible representations of automorphism groups of fields, Math. Z. 249 (2005), no. 1, p. 163-221.

[Tit74] J. TiTs - Homorphismes "abstraits" de groupes de Lie, in Symposia Mathematica, Vol. XIII (Convegno di Gruppi e loro Rappresentazioni, INDAM, Rome, 1972), Academic Press, London, 1974, p. 479-499. 

Mathematisches Institut, Seminars, (Y. TschINKel, ed.), p. 155-195

Universität Göttingen, 2004-05

\section{$\infty$-GROUPOIDS, STACKS, AND SEGAL CATEGORIES}

\section{Barwick}

Mathematisches Institut, Universität Göttingen, Bunsenstr. 3-5, 37073

Göttingen, Germany • E-mail : clarkbar@gmail.com

Résumé. Motivated by descent problems for $K$-theory and derived categories, and inspired by insights in the theory of infinite loop spaces, I introduce a robust theory of $(\infty, n)$-categories and $(\infty, n)$-stacks, and I formulate several important results and conjectures within this framework.

These are heavily revised and reorganized notes for a five-part series of talks I gave at the Mathematisches Institut Göttingen, in early December 2004. I thank Y. Tschinkel and M. Spitzweck for making my visit possible. I thank M. Spitzweck and G. Racinet for many discussions during which I was able to revise my presentation of the facts introduced in these notes. Finally, I thank J. P. May for inviting me to the University of Chicago for a week, where many of the revisions to these notes were made.

\section{Descent Problems for $\infty$-Categories.}

Let us be glad we don't work in algebraic geometry.-J. F. Adams. I should begin by apologizing for what will be at times very elementary and occasionally rigorless notes. When one is attempting to introduce a new formalism or piece of machinery, it is nothing more than good citizenship to begin by motivating an audience who otherwise would not have taken an interest in the formalism. This will be my sole focus in this talk. I intend to discuss two problems that exhibit a need for a more "complete" theory of descent. I will rather

December 2004 . 
frequently recite theorems and definitions that are well-known to everyone, and I will almost certainly offend members of the audience by omitting important but (for my purposes, at least) irrelevant details. Nevertheless, I would like to open with two problems that should be of interest to Göttingeners.

1.1 (A naïve view of $K$-theory). As I was originally to present this talk in a seminar ostensibly intended for talks on $L$-functions and the like, I feel I should begin by giving a brief history of an interesting problem, whose origins lie in the theory of $\zeta$-functions. In fact, this problem is by now very probably solved, but it may come as little surprise that there are aspects of the proof that seem less than ideal.

\subsubsection{Suppose :}

$F$ a number field with

$r_{1}$ real embeddings and

$r_{2}$ pairs of complex embeddings;

$\mathscr{O}_{F}$ the ring of integers of $F$;

$c_{F}$ the class numberof $F$.

Recall that the Dedekind $\zeta$-function

$$
\zeta_{F}(s)=\sum_{0 \neq \mathfrak{a} \triangleleft \mathscr{O}_{F}} \frac{1}{\#\left(\mathscr{O}_{F} / \mathfrak{a}\right)}
$$

is a natural generalization of Riemann's $\zeta$-function $\zeta=\zeta_{\mathbf{Q}}$, and is convergent for $\Re s>1$. As with Riemann's $\zeta$-function, $\zeta_{F}$ :

- can be extended to a meromorphic function on all of $\mathbf{C}$,

- has a simple pole at $s=1$, and

- satisfies a beautiful functional equation relating $\zeta_{F}(s)$ and $\zeta_{F}(1-s)$.

1.1.2. Examination of this functional equation reveals the following interesting facts :

- $\zeta_{F}$ has a zero of order $r_{1}+r_{2}-1$ at the origin.

- The first nonzero coefficient in a Taylor expansion about an integer $1-n$ for $n>0$ is the special value of $\zeta_{F}$ at $1-n$, denoted $\zeta^{\star}(1-n)$. Even for $n=1$, this special value contains some remarkable arithmetic information about the field $F$, as one can see from Dirichlet's Analytic Class Number Formula. Dirichlet defined a regulator map

$$
\rho_{F}^{D}: \mathscr{O}_{F}^{\times} / \mu_{F} \rightarrow \mathbf{R}^{r_{1}+r_{2}-1}
$$

which is a logarithmic embedding of the lattice $\mathscr{O}_{F}^{\times} / \mu_{F}$ into the vector space $\mathbf{R}^{r_{1}+r_{2}-1}$; the covolume of the image lattice is the Dirichlet regulator 
$D R_{F}$. Dirichlet's Analytic Class Number Formula then states that

$$
\zeta^{\star}(0)=-\frac{c_{F}}{\# \mu_{F}} D R_{F} .
$$

- The order of vanishing of $\zeta_{F}$ at $1-n$, for $n>1$, is

$$
d_{n}= \begin{cases}r_{1}+r_{2} & \text { if } n \text { is odd } \\ r_{2} & \text { if } n \text { is even }\end{cases}
$$

Moreover the values of $\zeta_{F}$ at the positive integers are determined by its special values at the negative integers.

1.1.3. Since they determine the values of the $\zeta$ function at all integers, one may well expect that the special values $\zeta^{\star}(1-n)$ contain even more arithmetic data about $F$ for $n>1$. To try to generalize Dirichlet's formula appropriately, it seems necessary to introduce some other arithmetic invariants of rings, and to play with them. This leads us to $K$-theory, to which we now turn.

1.1.4. Suppose $R$ is a commutative, unital ring. Grothendieck defined the $K$-theory $K_{0}(R)$ to be the free abelian group generated by the isomorphism classes of finitely generated projective $R$-modules, modulo the relation that for any short exact sequence

$$
0 \rightarrow M \rightarrow P \rightarrow N \rightarrow 0,
$$

$[M]+[N]=[P]$. Since any short exact sequence of finitely generated projective $R$-modules is split, this relation amounts to the equation $[M]+[N]=[M \oplus N]$. One easily verifies that two projective $R$-modules are equal as elements in $K_{0}(R)$ iff they are stably isomorphic, in the sense that there exists an isomorphism between $M \oplus R^{\oplus n}$ and $N \oplus R^{\oplus n}$ for some integer $n \geqslant 0$.

Example 1.1.5. $\quad$ Easy : If $R$ is a principal ideal domain or a local ring, the rank function gives rise to an isomorphism $K_{0}(R) \rightarrow \mathbf{Z}$.

Hard : If $R$ is a Dedekind domain, then $K_{0}(R)$ is isomorphic to $\mathbf{Z} \oplus \mathrm{Cl}(R)$, where of course $\mathrm{Cl}(R)$ denotes the Dedekind class group of $R$.

1.1.6. For the purposes of reinterpreting and generalizing Dirichlet's formula, I actually require much more than $K_{0}$. The failure of certain moduli spaces to be representable in algebraic geometry shows that it is rarely enough to work with isomorphism classes of objects. One must be, in some sense, conscientious of their automorphisms. Since $K_{0}$ measures the failure of the uniqueness of representatives of stable isomorphism types of projective modules, there is a similar loss of information here; one would like also a measurement of the failure of the uniqueness of these isomorphisms. In other words, one should 
like to work not with a group, but perhaps with a groupoid whose objects are "stable" projective modules, and whose morphisms are somehow "stable" isomorphism between them. An invariant, $K_{1}$, would then be the "fundamental group" of this groupoid, classifying equivalence classes of automorphisms.

1.1.7. Historically, a definition for $K_{1}$ actually appeared before that of $K_{0}$, as $K_{1}$ arises quite naturally in cobordism theory, and its definition is quite simple. The most efficient definition is as the abelianization of the infinite general linear group :

$$
K_{1}(R)=\mathrm{GL}(R) /[\mathrm{GL}(R), \mathrm{GL}(R)] .
$$

The commutator $[\mathrm{GL}(R), \mathrm{GL}(R)]$ is, by a lemma of Whitehead, equal to the subgroup $E(R)$ of $\mathrm{GL}(R)$ generated by the elementary matrices.

Example 1.1.8. Easy : The determinant yields an isomorphism

$$
K_{1}(R) \rightarrow R^{\times} \oplus \mathrm{SL}(R) / E(R) .
$$

Hard : If $R$ is the ring of integers in a number field, then in fact $S L(R) / E(R)$ is trivial. (This follows from the Bass-Milnor-Serre solution of the congruence subgroup problem for $\mathrm{SL}_{n}$.) Thus for a number field $F$, $K_{1}\left(\mathscr{O}_{F}\right)=\mathscr{O}_{F}^{\times}$.

1.1.9. It is now possible to reinterpret Dirichlet's formula, viz. :

$$
\zeta^{\star}(0)=-\frac{\left|K_{0}\left(\mathscr{O}_{F}\right)^{\text {tors }}\right|}{\left|K_{1}\left(\mathscr{O}_{F}\right)^{\text {tors }}\right|} D R_{F} .
$$

1.1.10. It was Quillen who realized that $K_{0}$ and $K_{1}$ - or even the groupoid of which they are $\pi_{0}$ and $\pi_{1}$-would not suffice. Quillen realized that an automorphism might be equivalent to another in a multitude of ways, and these differences are important, for example, to give suitable long exact sequences. To make all of this work, Quillen said, it is necessary to work with some much more robust : a simplicial set. The 0 -cells of such a simplicial set should be the "stable" projective modules, the 1-cells should be the "stable" isomorphisms between them, the 2 -cells should be equivalences between these isomorphisms, and so on. In other words, Quillen was looking for an $\infty$-groupoid, which he imagined as a simplicial set. From this, he believed, it should be possible to extract the $K$-groups as the homotopy groups of this simplicial set. A variant of the following definitions, which appeared in print for the first time in a paper by Waldhausen, was in fact known to Quillen long before his $Q$ construction. 
Definition 1.1.11. A category with cofibrations $\mathbf{A}=(\mathbf{A}$, cof $\mathbf{A})$ consists of a pointed category $\mathbf{A}$ and a subcategory cof $\mathbf{A}$ that contains $\iota \mathbf{A}$, whose morphisms are called cofibrations such that the following axioms hold.

- For any object $X$ of $\mathbf{A}$, the unique morphism $\star \rightarrow X$ is a cofibration.

- For any cofibration $X>Y$ and any morphism $X \rightarrow Z$, the pushout

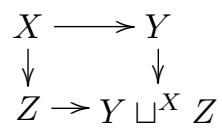

exists, and the canonical morphism $Z \rightarrow Y \sqcup^{X} Z$ is a cofibration.

Definition 1.1.12. A morphism $Y \rightarrow Z$ of $\mathbf{A}$ for which there exists a cofibration $X \rightarrow Y$ such that the square<smiles>[Y][Y]1[V][V][Tl]1</smiles>

is a pushout square is called a fibration, and in this case, such an object $Z$ (which is unique up to a canonical isomorphism) will be suggestively denoted by $Y / X$, and the sequence $X \rightarrow Y \rightarrow Y / X$ is called a cofibration sequence.

Example 1.1.13. Any exact category is a category with cofibrations in which the cofibrations are the exactly the admissible monomorphisms. Thus for a ring $R$, the category of finitely generated projective $R$-modules is a category with cofibrations.

Example 1.1.14. A pointed category with all finite colimits is a category with cofibrations in which every morphism is a cofibration.

Definition 1.1.15. For any nonnegative integer $p$, let $p$ denote also the category $[0 \rightarrow 1 \rightarrow \ldots \rightarrow p]$. I will use the functor category $p^{1}$, which is sometimes referred to as the "arrow category" of $p$. Its objects are pairs $(i, j)$ with $0 \leqslant i \leqslant$ $j \leqslant p$. Suppose now A a category with cofibrations; then a functor $X: p^{1} \rightarrow \mathbf{A}$ is said to be a $p$-filtered object of $\mathbf{A}$ iff the following axioms are satisfied.

- For every $0 \leqslant j \leqslant p, X(j, j)=\star$.

- For every $0 \leqslant i \leqslant j \leqslant k \leqslant p$, the morphism $X(i, j) \rightarrow X(i, k)$ is a cofibration.

- For every $0 \leqslant i \leqslant j \leqslant k \leqslant p$, the square

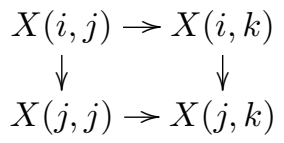


is a pushout.

Thus the sequence $X(i, j) \rightarrow X(i, k) \rightarrow X(j, k)$ is a cofibration sequence, and to give a $p$-filtered object of $\mathbf{A}$ is to give a sequence of cofibrations

$$
X(0,1) \rightarrow X(0,2) \rightarrow \cdots \rightarrow X(0, p)
$$

together with a choice of subquotients $X(i, j)=X(0, i) / X(0, j)$.

Definition 1.1.16. For any nonnegative integer $p$ and any category with cofibrations $\mathbf{A}$, let $S_{p} \mathbf{A}$ denote the full subcategory of the functor category $\mathbf{A}^{p^{1}}$ spanned by the $p$-filtered objects. This gives a simplicial category $S_{\bullet} \mathbf{A}$, i.e., a simplicial object in the category of categories. Thus Obj $S_{\bullet} \mathbf{A}$ is a simplicial set, with a unique 0 -cell. (It is canonically pointed.) One then defines $K(\mathbf{A})$, the $K$-theory of the category with cofibrations $A$ as the simplicial set $\operatorname{Ex}^{\infty} \Omega\left(\operatorname{Obj} S_{\bullet} \mathbf{A}\right)$.

Exercise 1.1.17 (Easy). Use Kan's loop description of the fundamental group of a space with only one zero simplex to show that if $R$ is a ring, and $\mathbf{A}$ is the category with cofibrations of finitely generated projective $R$-modules, then $K_{0}(R)$ is in fact isomorphic (naturally, in fact) to $\pi_{0} K(\mathbf{A})=\pi_{1}$ Obj $S_{\bullet} \mathbf{A}$.

Example 1.1.18. Hard: In fact $K_{1}(R)$ is isomorphic to $\pi_{1} K(\mathbf{A})$. Probably the quickest way to write a proof is to observe the following.

Key : Quillen's $K$-theory of an exact category (using the $Q$-construction) and the one I have given for the corresponding category with cofibrations are naturally homotopy equivalent.

Definition 1.1.19. One thus defines the higher algebraic $K$-theory of a ring $R$ by $K_{i}(R)=\pi_{i} K(\mathbf{A})$, where $\mathbf{A}$ is the category with cofibrations of finitely generated projective $R$-modules.

1.1.20. This description, though perhaps a little abstract, has several benefits, which together make it my favorite approach to algebraic $K$-theory. I list some.

- This approach clearly exhibits the categorical origins of higher $K$ theory. We see that $K$-theory has nothing to do with additivity.

- This description can be used to give more than a mere simplicial set : $K(\mathbf{A})$ is in fact an infinite loop space. This can be easily seen from this construction. Indeed, by naturality, the $S_{\bullet}$ construction extends to (multi)simplicial categories with cofibrations. One can define the cofibrations of $S_{\bullet} \mathbf{A}$ in a very natural way, and therefore it is possible to iterate the construction. Now simply let $S_{\bullet}^{(m)} \mathbf{A}$ be the application of $S_{\bullet}$ to $S_{\bullet}^{(m-1)} \mathbf{A}$. 
It is easy to see that the inclusion of the $1-$ cell $\mathrm{Obj} \mathbf{A}$ gives rise to a natural map

$$
S^{1} \wedge \operatorname{Obj} \mathbf{A} \rightarrow S^{1} \wedge \operatorname{Obj} S_{\bullet} \mathbf{A}
$$

It follows that the spaces $\left(\mathbf{K}^{\prime} \mathbf{A}\right)_{m}=\operatorname{diag}\left(S_{\bullet}^{(m)} \mathbf{A}\right.$ define a spectrum, and, by permuting the application of the $S_{\bullet}$, one effortlessly makes it a symmetric spectrum. The $K$-theory spectrum KA is then the loop spectrum of this spectrum. KA is quickly seen to be an $\Omega$-spectrum, and so it follows that $K \mathbf{A}$ is the infinite loop space of this spectrum.

- Still more is true : if $\mathbf{A}$ is a symmetric monoidal category, a multiplicative structure on KA is induced, making KA into a symmetric ring spectrum.

- This construction can also be used in a subtler way (yielding the $w S$. construction), which can be applied to Waldhausen categories, which come equipped with certain "weak equivalences." Rather than give the technical definition, let it suffice to say that the full subcategory of cofibrant-fibrant objects of a pointed, proper closed model category forms a Waldhausen category, and all known examples of Waldhausen categories can be constructed in this way. This machine can therefore be applied to the category of bounded chain complexes in an abelian category $A$ with an injective model structure, in which the weak equivalences are quasi-isomorphisms, and the cofibrations are degreewise admissible monomorphisms. Again this $S_{\bullet}-$ construction of Waldhausen can be made, and the resulting $K$-theory is, by a theorem of Waldhausen, Gillet, and Thomason, homotopy equivalent to the $K$-theory of $A$, viewed as a category with cofibrations as above.

1.1.21. Borel demonstrated that for a number field $F, K_{m}\left(\mathscr{O}_{F}\right)$ for $m$ even and positive is finite.

1.1.22. Generalizing Dirichlet's regulator map, Borel constructed higher regulator maps

$$
\rho_{F}^{B}: K_{2 n-1}\left(\mathscr{O}_{F}\right) \rightarrow \mathbf{R}^{d_{n}}
$$

for $n>0$ (where, recall, $d_{n}$ is the order of vanishing of $\zeta_{F}$ at $1-n$ ), and showed that the kernel is finite, and the image is a lattice in $\mathbf{R}^{d_{n}}$, whose covolume is the Borel regulator $B R_{F}$. When $n=0$, the Borel regulator and the Dirichlet regulator correspond. Borel used this regulator to show that:

- $K_{2 n-1}$ has rank $d_{n}$, and

- $\zeta_{F}^{\star}(1-n)=q_{n} B R_{F}$ for some rational number $q_{n}$.

This last result is clearly a weakened generalization of Dirichlet's theorem. 
Conjecture 1.1.23 (Lichtenbaum). Lichtenbaum proposed in $19^{\text {r7 }}$ a strict generalization of Dirichlet's theorem, namely,

$$
\zeta_{F}^{\star}(1-n)= \pm \frac{\# K_{2 n-1}\left(\mathscr{O}_{F}\right)^{\text {tors }}}{\# K_{2 n-2}\left(\mathscr{O}_{F}\right)^{\text {tors }}} B R_{F},
$$

for any $n>0$, up to a power of 2 .

1.1.24. As a consequence of Wiles' proof of the main conjecture of Iwasawa theory, we have the following result. Suppose that $F$ is a totally real number field, $n$ a positive even integer. Then

$$
\zeta_{F}^{\star}(1-n)= \pm \frac{\#\left(\prod_{p} H_{\text {ét }}^{2}\left(\mathscr{O}_{F}, \mathbf{Z}_{p}(n)\right)\right)}{\# H^{0}(F, \mathbf{Q} / \mathbf{Z}(n))}
$$

up to powers of 2 . Thus a good approach might be to make use of some relationship between $K$-theory and étale cohomology.

1.1.25. Such a relationship already exists : Grothendieck's general theory of Chern classes gives the étale Chern characters for each prime number $p$

$$
\chi_{i, n}^{p}: K_{2 n-i}\left(\mathscr{O}_{F}\left[\frac{1}{p}\right]\right) \rightarrow H_{\text {ét }}^{i}\left(\mathscr{O}_{F}, \mathbf{Z}_{p}(n)\right),
$$

which have been shown to be surjective for $i=1,2,2 n>i$, and either $p$ odd or $\sqrt{-1} \in F$ by Soulé and Dwyer-Friedlander.

Conjecture 1.1.26 (Quillen-Lichtenbaum). The étale Chern character $\chi_{1, n}^{p}$ is an isomorphism if $p$ is an odd prime.

1.1.27. An immediate corollary of the Quillen-Lichtenbaum conjecture is that the Lichtenbaum conjecture is true for a totally real number field. It would therefore be very nice to have a proof of this conjecture. In fact, the QuillenLichtenbaum conjecture is a consequence of the Kato conjecture, which is the assertion that a particular Galois symbol is always an isomorphism. Nevertheless, there is something unsettling about this conjecture. The Chern character is produced by a general result in homological algebra, which is somewhat mysterious. Fortunately, we can rephrase this conjecture in a manner that is more concrete, and more general.

\section{Conjecture 1.1.28 (Quillen-Lichtenbaum, General Concrete Ver- sion)}

Suppose $F$ a field, $G_{F}$ its absolute Galois group of cohomological dimension d. Suppose $\ell$ is a prime different from the characteristic of $F$. Then there is a natural morphism of ring spectra

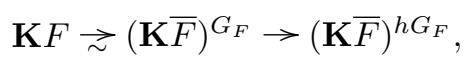


where $(\mathbf{K} \bar{F})^{h G_{F}}$ is the homotopy fixed point set $\underline{\operatorname{Mor}}_{G}(E G, \mathbf{K} \bar{F})$. The statement of the conjecture is : the induced morphism

$$
\widehat{\mathbf{K F}}_{\ell} \rightarrow\left(\widehat{\mathbf{K} \bar{F})^{h G_{F}}}{ }_{\ell}\right.
$$

induces a "co-d" weak equivalence, i.e., it induces an isomorphism on all homotopy groups $\pi_{i}$ for $i>d$. Since there is a spectral sequence converging to $\pi_{p+q}\left((\mathbf{K} \bar{F})^{h G_{F}}\right)$ whose $E_{2}$ term is

$$
\left.E_{2}^{p, q}=H^{-p}\left(G_{F}, \pi_{q}(\widehat{(\mathbf{K} \bar{F}})_{\ell}\right)\right),
$$

this spectral sequence must converge to $\pi_{p+q}\left(\widehat{\mathbf{K F}}_{\ell}\right)$ for $p+q>d$.

1.1.29. A simple question is the following: Is this spectral sequence in any sense a descent spectral sequence for the Galois descent of $K$-theory? It seems to be, but how can one make this precise?

1.2 (Chain complexes when the base varies). I have been informed that in the model category seminar, the projective model structure on the category of unbounded $R$-modules for a ring $R$ has been constructed. I will denote this category $\mathbf{C p l x}(R)$. I am interested in what happens when the base varies.

1.2.1. Suppose $f: R \rightarrow S$ a homomorphism of rings. Then the functor

$$
f^{\star}=-\otimes_{R} S: \mathbf{C p l x}(R) \rightarrow \mathbf{C p l x}(S)
$$

has a right adjoint $f_{\star}$, which is the forgetful functor. Observe that $f_{\star}$ preserves objectwise epimorphisms and quasi-isomorphisms, so $\left(f^{\star}, f_{\star}\right)$ defines a Quillen adjunction.

Exercise 1.2.2. Show that a ring homomorphism $f$ induces a Quillen equivalence $\left(f^{\star}, f_{\star}\right)$ iff it is an isomorphism.

1.2.3. One can think of the assignment $R \mapsto \mathbf{C p l x}(R)$ as a kind of "presheaf" in model categories on the category of affine schemes. Such a presheaf is sometimes called a left Quillen presheaf. The assignment $R \mapsto \mathbf{D}(R)=\operatorname{Ho} \mathbf{C p l x}(R)$ is a kind of "presheaf" in categories. One can ask whether such a thing is a stack. This would indicate, in particular, that it is possible to "glue" complexes up to quasi-isomorphism.

Definition 1.2.4. Suppose $C$ a category. Then a pseudofunctor $L$ on $C$ (taking values in the category of categories) consists of the following data :

- an assignment of a category $L X$ to any object $X \in C$, 
— an assignment of a functor $L f: L X \rightarrow L Y$ to any morphism $f: X \rightarrow Y$ in $C$, and

— an assignment of a natural isomorphism $\gamma_{g, f}: L(g f) \rightarrow L g \circ L f$ to any pair of morphisms

$$
X \stackrel{f}{\longrightarrow} Y \stackrel{g}{\longrightarrow} Z
$$

in $C$,

subject to the following axioms :

— for any morphism $f: X \rightarrow Y$ in $C, \gamma_{f, \mathbf{1}_{X}}=\mathbf{1}_{L f}=\gamma_{\mathbf{1}_{Y}, f}$, and

— for any triple of morphisms

$$
A \stackrel{f}{\longrightarrow} B \stackrel{g}{\longrightarrow} C \stackrel{h}{\longrightarrow} D
$$

the diagram

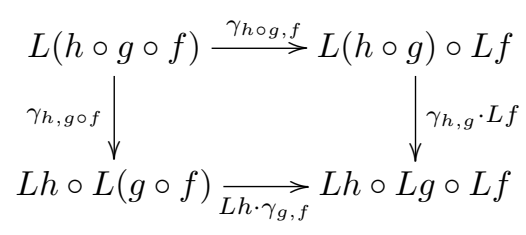

commutes.

Definition 1.2.5. A stack (in categries) $L$ on a site $(T, \tau)$ is a contravariant pseudofunctor on $T$ satisfying the following properties.

- $L$ is a separated prestack : for any object $X$ of $\mathscr{S}$, and for any pair of objects $F, G \in L X$, the presheaf of sets on the site $\mathscr{S} / X$ that assigns to any $U \stackrel{i}{\longrightarrow} X$ the set $\operatorname{Mor}_{L U}\left(\left.F\right|_{U},\left.G\right|_{U}\right)$ is a sheaf (i.e., one can glue morphisms). 
- For any covering family $\left\{U_{i} \rightarrow U\right\}_{i \in I}$, any family of objects $\left\{F_{i} \in\right.$ $\left.L U_{i}\right\}_{i \in I}$, and any family of isomorphisms $\theta_{i j}:\left.\left.F_{i}\right|_{U_{i} \times_{U} U_{j}} \rightarrow F_{j}\right|_{U_{i} \times_{U} U_{j}}$ satisfying the cocycle condition, i.e., the commutativity of

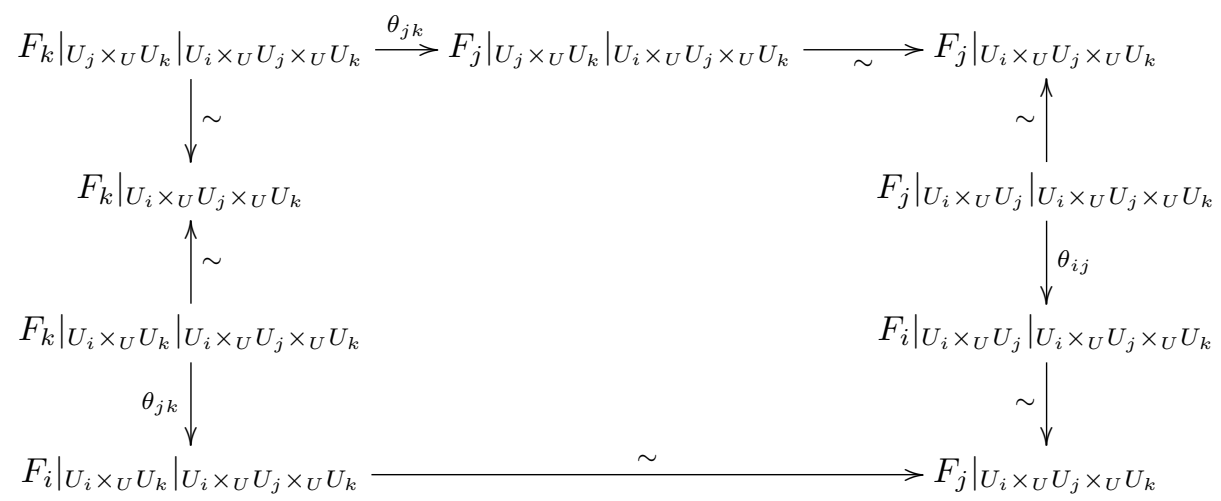

there exist $F \in L U$ and isomorphisms $\theta_{i}:\left.F\right|_{U_{i}} \rightarrow F_{i}$ such that the diagram

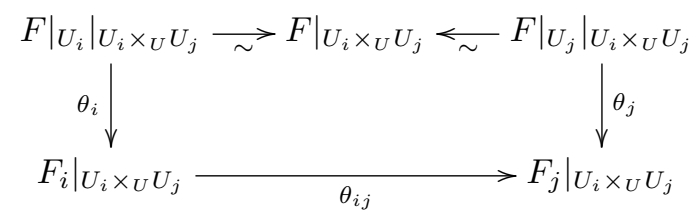

commutes (i.e., one can glue objects).

1.2.6. Is, then, the assignment $R \mapsto \mathbf{D}(R)$ a stack on the Zariski site of affine schemes? Consider what this would mean for an affine scheme $X$ and an affine open cover $\left\{U_{0}, U_{1}, U_{2}, U_{3}\right\}$ thereof. Unpacking the above definition carefully, we see that in order for $\mathbf{D}$ to be a stack, then, given the following data:

- for each $0 \leqslant i \leqslant 3$, complexes of $H^{0}\left(\mathscr{O}_{U_{i}}\right)$-modules $C_{i}^{\bullet}$,

- for each $0 \leqslant i \leqslant j \leqslant 3$, a quasi-isomorphism $f_{i j}:\left.\left.C_{i}^{\bullet}\right|_{U_{j}} \underset{\sim}{\sim} C_{j}^{\bullet}\right|_{U_{i}}$, and

- for each $0 \leqslant i \leqslant j \leqslant k \leqslant 3$, a chain homotopy $h_{i j k}: f_{j k} \circ f_{i j} \simeq f_{i k}$, there must exist a complex of $H^{0}\left(\mathscr{O}_{X}\right)$-modules $C^{\bullet}$ such that $\left.C^{\bullet}\right|_{U_{i}}$ is quasiisomorphic to $C_{i}$. But observe that if this were the case, then the homotopies 
$h_{i j k}$ could be chosen so that the following square of homotopies commutes :

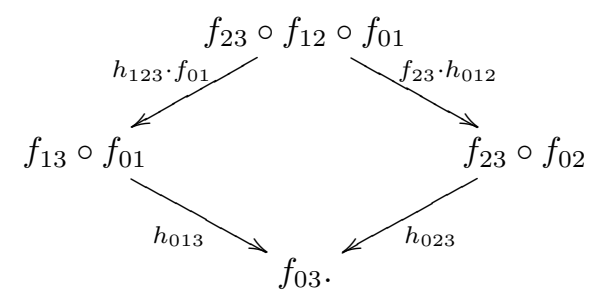

Imagine the resulting combinatorics if we had had an open cover of cardinality 5 . Then there would be 6 diagrams not given as part of the data! This certainly seems to be too much to hope for, and it is.

Exercise 1.2.7. Show that $\mathbf{D}$ is not a stack. (Hint : an easy example can be given using an open cover of cardinality 4 of $\mathbf{A}_{k}^{4}$.) Grothendieck said of the objects of the derived category that they were "de nature essentiellement non recollables."

1.2.8. The problem with $\mathbf{D}$ is, in a very precise sense, the same problem as the problem with $K_{0}$. We have lost "higher homotopical" data in passing to $K_{0}$ and the to the derived category. But how do write down a descent condition for left Quillen presheaves?

1.3 (Toward $\infty$-groupoids). Having given two motivating examples, I now turn to the design of a suitable theory of $\infty$-categories that will yield the proper setting in which to formulate (and, eventually, answer) the questions of descent. The notion of an $\infty$-category is one that, for the moment, I intend to use heuristically; morally, an $\infty$-category consists of a set ${ }^{(1)}$ of objects, a set of morphisms between any two objects, sets of 2 -morphisms between any two morphisms, 3-morphisms between any two 2-morphisms, and so on. The $n$-morphisms should be composable, up to a coherent $(n+1)$-morphism, and the composition law should be associative up to a natural coherent $(n+1)$ morphism.

I shall consider only $(\infty, n)$-categories - i.e., $\infty$-categories in which the $i$ morphisms are invertible up to an $(i+1)$-morphism for all $i>n-$, as these are the kinds of $\infty$-categories that typically arise in algebraic geometry. Making this notions precise for $n=0$ will be the focus of this section. In order

\footnotetext{
(1) Here I neglect any set-theoretic difficulties, which are aptly handled by the use of closed model categories and Grothendieck universes.
} 
to motivate the definition, it is convenient to discuss and interpret the nerve construction of Grothendieck.

1.3.1. Let Cat denote the category of (small) categories; observe that $\Delta$ is isomorphic to the full subcategory of Cat spanned by the categories $p=$ $[0 \rightarrow 1 \rightarrow \ldots \rightarrow p]$. Composing the ordinary enrichment functor

$$
\text { Mor : } \text { Cat }^{\text {op }} \times \text { Cat } \rightarrow \text { Set }
$$

with the natural functor

$$
\Delta^{\mathrm{op}} \times \mathbf{C a t} \rightarrow \mathrm{Cat}^{\mathrm{op}} \times \mathbf{C a t},
$$

yields, by adjunction, a functor Cat $\rightarrow s$ Set, called the nerve functor, $\nu_{\bullet}$.

Proposition 1.3.2. The functor $\nu_{\bullet}$ is fully faithful and has a left adjoint.

Proof. One recovers a category $C$ from its nerve $\nu_{\bullet} C$ in the following manner. Its set of objects is $\nu_{0} C$; for any two objects $X$ and $Y$ of $C$, the set $\operatorname{Mor}_{C}(X, Y)$ is the fibre of the morphism $\left(d_{1}, d_{0}\right): \nu_{1} C \rightarrow \nu_{0} C \times \nu_{0} C$ over the pair $(X, Y)$. The compostion law is given by the composite map

$$
\nu_{1} C \times{ }_{\nu_{0}} C \nu_{1} C \underset{\sim}{\sim} \nu_{2} C \stackrel{d_{1}}{\rightarrow} \nu_{1} C .
$$

It is now easy to check that the natural map

$$
\operatorname{Mor}_{\mathbf{C a t}}(C, D) \rightarrow \operatorname{Mor}_{s \operatorname{Set}}\left(\nu_{\bullet} C, \nu_{\bullet} D\right)
$$

is a bijection.

Lemma 1.3.3. If $C$ is a small category, then $\nu_{\bullet} C$ is a 2-coskeleton. That is, for any simplicial set $X_{\bullet}$, the natural map

$$
\operatorname{Mor}_{s} \operatorname{Set}\left(X_{\bullet}, \nu_{\bullet} C\right) \rightarrow \operatorname{Mor}_{s} \operatorname{Set}\left(\operatorname{sk}_{2} X_{\bullet}, \nu_{\bullet} C\right)
$$

is a bijection.

Proof. Using the previous proposition, one sees that this lemma holds when $X$. is a standard simplicial set $\Delta^{n}$. Since any simplicial set is a colimit of standard simplicial sets, the result follows.

Proposition 1.3.4. The nerve of a small category $C$ is a Kan simplicial set iff $C$ is a groupoid. Moreover, a functor $C \rightarrow D$ between groupoids is an equivalence iff the induced morphism $\nu_{\bullet} C \rightarrow \nu_{\bullet} D$ is a weak equivalence. 
Proof. By the lemma, it suffices to verify the Kan condition for morphisms $\Lambda^{n}[k] \rightarrow \nu \bullet C$ when $n \leqslant 3$. Proceeding case by case, one sees that this is equivalent to the invertibility of any morphism. The final sentence of the proposition follows from the observation that for fibrant simplicial sets, homotopies and simplicial homotopies are the same.

Theorem 1.3.5 (Thomason). Cat is a closed model category, wherein a functor $F: C \rightarrow D$ is a weak equivalence or a fibration iff the induced morphism

$$
\operatorname{Ex}^{2} \nu_{\bullet} C \rightarrow \operatorname{Ex}^{2} \nu_{\bullet} D
$$

of simplicial sets is so.

About the Proof. Thomason demonstrated this directly, by presenting generating sets of cofibrations and of trivial cofibrations, and using the small object argument. Unfortunately, Thomason's proposed class of cofibrations is not stable under retracts, an error noticed and repaired in a recent note of Cisinski.

1.3.6. The interpretation I have in mind for these facts is the following. Fibrant simplicial sets are models for weak $\infty$-groupoids; a 0 -simplex of a simplicial set should be viewed as an object of the $\infty$-groupoid; a 1 -simplex $y$ is a 1 -isomorphism from $d_{1} y$ to $d_{0} y$; a 2-simplex is a 2-morphism; etc. Of course, this particular avatar of the notion of $n$-morphism may be unfamiliar, since under this interpretation, $n$-morphisms do not have only a source and target, but have $(n+1)$ faces instead.

The role of the Kan condition is to guarantee composability. Indeed, given two 1-morphisms of an $\infty$-groupoid, $A \rightarrow B \rightarrow B$, there should exist a "composite up to homotopy," $A \rightarrow C$; more precisely, for any pair of 1 -simplices $x_{1}$ and $x_{2}$ with $d_{0} x_{1}=d_{1} x_{2}$, there should exist a 2 -simplex $x$ with $d_{1} x=x_{1}$ and $d_{2} x=x_{2}$ - the composite up to homotopy is then $d_{0} x$. This is precisely the Kan condition in dimension 2. In higher dimensions, the Kan condition guarantees up-to-homotopy composability for certain configurations of $(n+1)$ $n$-morphisms. This leads to the following definition.

Definition 1.3.7. A (weak) $\infty$-pregroupoid is a simplicial set. A (weak) $\infty$-groupoid is a fibrant simplicial set.

Example 1.3.8. If $R$ is a ring (commutative, unital), and $\mathbf{A}$ is the category with cofibrations of finitely generated projective $R$-modules, then $K(\mathbf{A})$ is an $\infty$-groupoid 
1.4 (S-categories and simplicial localization). S-categories are categories enriched over simplicial sets, and therefore fibrant $\mathbf{S}$-categories can be viewed as models for certain $(\infty, 1)$-categories. The simplicial localization construction of Dwyer and Kan gives a canonical way to convert categories with weak equivalences into $\mathbf{S}-$ categories.

Definition 1.4.1. An $\mathbf{S}-$ category is a category enriched over $s$ Set. Functors that preserve the simplicial structure are called $\mathbf{S}$-functors. The category of small $\mathbf{S}$-categories is denoted $\mathbf{S}-\mathbf{C a t}$.

1.4.2. Any ordinary category can be viewed as an $\mathbf{S}$-category in the obvious way. This defines a functor

$$
\text { Cat } \rightarrow \text { S }- \text { Cat }
$$

whose left adjoint is the functor that to any $\mathbf{S}$-category $\mathbf{C}$ assigns the category whose objects are exactly those of $\mathbf{C}$ and whose Mor-set from an object $X$ to an object $Y$ is the set $\pi_{0} \operatorname{Mor}_{\mathbf{C}}(X, Y)$. For brevity, denote this left adjoint simply by $\pi_{0}$.

Definition 1.4.3. An $\mathbf{S}$-equivalence is an $\mathbf{S}$-functor $F: \mathbf{C} \rightarrow \mathbf{D}$ that satisfies the following conditions.

- (Full faithfulness) For any objects $X$ and $Y$ of $\mathbf{C}$, the induced morphism or simplicial sets $\operatorname{Mor}_{\mathbf{C}}(X, Y) \rightarrow \operatorname{Mor}_{\mathbf{D}}(F X, F Y)$ is a weak equivalence.

- (Essential surjectivity) The induced functor $\pi_{0} \mathbf{C} \rightarrow \pi_{0} \mathbf{D}$ is essentially surjective.

Proposition 1.4.4 (Bergner). There exists a cofibrantly generated closed model structure on $\mathbf{S}$ - Cat with the following properties.

- The weak equivalences are exactly the $\mathbf{S}$-equivalences.

- The fibrations are those $\mathbf{S}$-functors $F: A \rightarrow B$ such that

- the induced morphisms $\operatorname{Mor}_{A}(x, y) \rightarrow \operatorname{Mor}_{B}(F x, F y)$ are fibrations for every pair of objects $(x, y)$, and

- for any object $a^{\prime}$ of $A$, any object $b$ of $B$, and any equivalence $e: F a^{\prime} \rightarrow b$, there exists an object $a$ of $A$ and an equivalence $d: a^{\prime} \rightarrow a$ such that $F d=e$.

About the Proof. Such a model structure has been believed to exist for some time now. Dwyer and Kan sketched a faulty proof, by providing generating sets of cofibrations and of trivial cofibrations. Unfortunately, their suggested generating trivial cofibrations are not weak equivalences. Bergner repaired this 
fault in the overture to her thesis, providing correct generating sets of cofibrations and of generating cofibrations and proving the existence of this model structure directly, by means of the small object argument.

Corollary 1.4.5. The fibrant objects of $\mathbf{S}-\mathbf{C a t}$ are exactly the categories enriched in $\infty$-groupoids.

1.4.6. A category enriched in $\infty$-groupoids can be viewed as an $(\infty, 1)$-category, wherein the $n$-morphisms are the $(n-1)$-cells of the Mor-sets. Observe that the morphisms of such $(\infty, 1)$-categories are strictly composable, and their composition law is strictly associative, so one might correctly call these strict $(\infty, 1)$-categories (or, more precisely, but altogether less linguistically practical, "weak- $\infty$-strict-1-categories"). The basic source of examples is the simplicial localization, to which I now turn.

Definition 1.4.7. A quasihomotopical category $\mathbf{C}=(\mathbf{C}, w \mathbf{C})$ consists of a category $\mathbf{C}$ and a full subcategory $w \mathbf{C}$ - whose morphisms are called weak equivalences - satisfying the two-out-of-three axiom. A functor $F: \mathbf{C} \rightarrow \mathbf{D}$ is homotopical iff for any weak equivalence $f$ of $\mathbf{C}, F f$ is a weak equivalence of D.

Definition 1.4.8. Suppose $\mathbf{C}=(\mathbf{C}, w \mathbf{C})$ is a quasihomotopical category. For any pair of objects $X$ and $Y$ in $\mathbf{C}$ and any odd number $n>0$, I define a category $w \operatorname{Mor}_{\mathbf{C}}^{n}(X, Y)$. The objects of $w \operatorname{Mor}_{\mathbf{C}}^{n}(X, Y)$ are strings of morphisms

$$
X=X_{0} \leftarrow X_{1} \rightarrow X_{2} \leftarrow \cdots \rightarrow X_{n-1} \leftarrow X_{n}=Y
$$

such that each morphism $X_{2 i} \leftarrow X_{2 i+1}$ is contained in $w \mathbf{C}$. Morphisms between two such sequences are simply commutative diagrams of the form

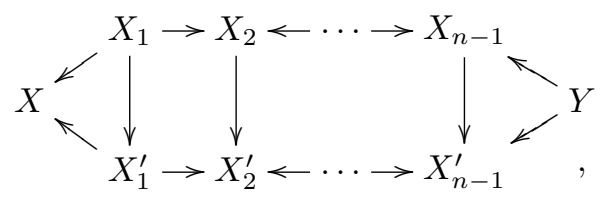

wherein the vertical maps are in $w \mathbf{C}$. The hammock localization of $\mathbf{C}$ is the $\mathbf{S}$-category $L \mathbf{C}$ whose objects are exactly those of $\mathbf{C}$, with

$$
\operatorname{Mor}_{L \mathbf{C}}(X, Y)=\operatorname{Ex}^{\infty} \operatorname{colim}_{n} \nu_{\bullet}\left(w \operatorname{Mor}_{\mathbf{C}}^{n}(X, Y)\right)
$$

for any objects $X$ and $Y$.

1.4.9. This construction is clearly pseudofunctorial with respect to homotopical functors. 
It is obvious that $\pi_{0} L \mathbf{C}$ is the Gabriel-Zisman localization $\mathbf{C}\left[w \mathbf{C}^{-1}\right]$. Moreover, there is a canonical $\mathbf{S}$-functor $\mathbf{C} \rightarrow L \mathbf{C}$ that is universal in a sense that is more or less immediate.

1.4.10. A priori, this construction seems nightmarish, because the colimit in question seems unmanageable. Fortunately, for model categories, it is not necessary to compute $w$ Mor $^{n}$ for $n>3$.

Lemma 1.4.11 (Dwyer-Kan). If $\mathbf{M}$ is a closed model category (CMC), then for any two objects $X$ and $Y$ of $\mathbf{M}$, the canonical morphism

$$
\nu_{\bullet}\left(w \operatorname{Mor}_{\mathbf{M}}^{3}(X, Y)\right) \rightarrow \operatorname{Mor}_{L \mathbf{M}}(X, Y)
$$

is a weak equivalence.

About the Proof. This is a more general fact that holds for any quasihomotopical category possessing a 3-arrow calculus, in the sense of Dwyer and Kan. It follows easily from the existence of functorial factorizations.

1.4.12. Recall that if $\mathbf{M}$ is a $\mathrm{CMC}$, then $s \mathbf{M}$ and $c \mathbf{M}$ each have a Reedy closed model structure. The cosimplicial resolution functor $q^{\bullet}$ is the composite

$$
\mathbf{M} \rightarrow c \mathbf{M} \rightarrow(c \mathbf{M})_{c}
$$

and, dually, the simplicial resolution functor $r_{\bullet}$ is the composite

$$
\mathbf{M} \rightarrow s \mathbf{M} \rightarrow(s \mathbf{M})_{f}
$$

Lemma 1.4.13. Suppose $X$ and $Y$ objects of a CMC M. Then the functors

$$
\operatorname{Mor}_{\mathbf{M}}\left(q^{\bullet} X,-\right): \mathbf{M}_{f} \rightarrow s \text { Set }
$$

and

$$
\operatorname{Mor}_{\mathbf{M}}\left(-, r_{\bullet} Y\right): \mathbf{M}_{c} \rightarrow s \text { Set }
$$

are homotopical.

Scholium 1.4.14 (Dwyer-Kan). Suppose $\mathbf{M}$ a CMC. There are $\mathbf{S}$ equivalences

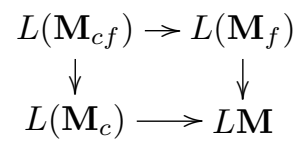


and there are natural weak equivalences of the simplicial sets

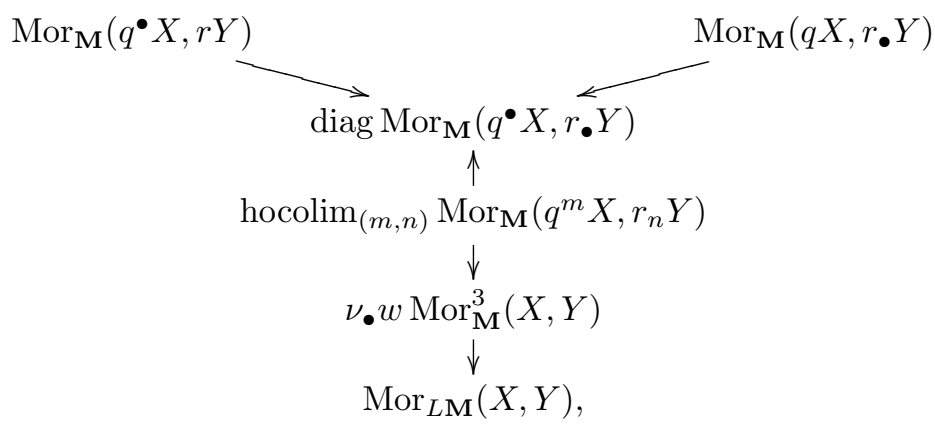

where $q$ and $r$ are the cofibrant and fibrant replacement functors in $\mathbf{M}$.

About the Proof. This chain of weak equivalences follows quickly from the Bousfield-Kan theory of homotopy colimits.

Corollary 1.4.15. If $\mathbf{M}$ is a simplicial closed model category, then the $\mathbf{S}$ category $\mathbf{M}_{c f}$ is $\mathbf{S}$-equivalent to LM.

Definition 1.4.16. Suppose $A$ an $\mathbf{S}$-category, $\kappa$ a regular cardinal. Then $A$ is $\kappa$-presentable if $A$ is cocomplete, and if there exists a $\kappa$-small sub-S-category $A_{c}$ spanned by $\kappa$-compact objects that generates $A$ by $\kappa$-filtered colimits. $A$ is said to be presentable if it is $\kappa$-presentable for some regular cardinal $\kappa$.

Theorem 1.4.17 (Simpson). Suppose $A$ an $\mathbf{S}$-category. Then the following are equivalent.

- There exists a cofibrantly generated $\mathrm{CMC} \mathbf{M}$ such that $A$ is $\mathbf{S}$ equivalent to $L \mathbf{M}$.

- A is presentable.

About the Proof. This is a very technical result, whose proof requires the use of the theory of Segal 1-categories, which I will introduce momentarily.

Example 1.4.18. For any ring $R$, the category $\mathbf{C p l x}(R)$ of unbounded chain complexes of $R$-modules is a combinatorial closed model category. The simplicial localization $L \mathbf{C p l x}(R)$ is therefore a presentable $\mathbf{S}$-category.

1.5 (Bousfield localization). There are two canonical ways to add weak equivalences to a closed model category in a minimal way. The existence proofs are often distractingly technical, so I shall not have much to say about them. 
Definition 1.5.1. Suppose $\mathbf{M}$ a CMC, and suppose $C$ a class of morphisms in $\mathbf{M}$.

- The left Bousfield localization of $\mathbf{M}$ with respect to $C$ is a $\mathrm{CMC} L_{C} \mathbf{M}$ equipped with a left Quillen functor $\mathbf{M} \rightarrow L_{C} \mathbf{M}$ that is initial among all left Quillen functors $F: \mathbf{M} \rightarrow \mathbf{N}$ with the property that for $f \in C, F f$ is a weak equivalence.

- The left Bousfield localization of $\mathbf{M}$ with respect to $C$ is a CMC $R_{C} \mathbf{M}$ equipped with a right Quillen functor $\mathbf{M} \rightarrow L_{C} \mathbf{M}$ that is initial among all left Quillen functors $F: \mathbf{M} \rightarrow \mathbf{N}$ with the property that for $f \in C, F f$ is a weak equivalence.

Proposition 1.5.2 (Cole). Suppose $M$ a category with two model structures $\mathbf{M}$ and $\mathbf{M}^{\prime}$ such that $w \mathbf{M} \subset w \mathbf{M}^{\prime}$ and fibM $\subset$ fibM $\mathbf{M}^{\prime}$. Then the right Bousfield localization $R_{w \mathbf{M}^{\prime}} \mathbf{M}$ exists; the underlying category of $R_{w \mathbf{M}^{\prime}} \mathbf{M}$ is again $M$, and $w\left(R_{w \mathbf{M}^{\prime}} \mathbf{M}\right)=w \mathbf{M}^{\prime}$ and $\operatorname{fib}\left(R_{w \mathbf{M}^{\prime}} \mathbf{M}\right)=$ fibM.

Likewise, if $N$ is a category with two model structures $\mathbf{N}$ and $\mathbf{N}^{\prime}$ such that $w \mathbf{N} \subset w \mathbf{N}^{\prime}$ and cof $\mathbf{N} \subset$ cof $\mathbf{N}^{\prime}$, then the left Bousfield localization $L_{w} \mathbf{N}^{\prime} \mathbf{N}$ exists; the underlying category of $L_{w \mathbf{N}^{\prime}} \mathbf{N}$ is again $N$, and $w\left(L_{w \mathbf{N}^{\prime}} \mathbf{N}\right)=w \mathbf{N}^{\prime}$ and $\operatorname{cof}\left(L_{w \mathbf{N}^{\prime}} \mathbf{N}\right)=\operatorname{cof} \mathbf{N}$.

Proof. This proof is left as an easy exercise in the axioms of closed model categories.

Definition 1.5.3. Suppose $\mathbf{C}$ a quasihomotopical category; suppose $S$ a class of morphisms thereof; and suppose $K$ a class of objects thereof.

- An object $X$ of $\mathbf{C}$ is $\mathbf{S}$-local (resp., $\mathbf{S}$-colocal if for any element $A \rightarrow B$ of $S$, the induced morphism $\operatorname{Mor}_{L \mathbf{C}}(B, X) \rightarrow \operatorname{Mor}_{L \mathbf{C}}(A, X)$

(resp., the induced morphism $\operatorname{Mor}_{L \mathbf{C}}(X, A) \rightarrow \operatorname{Mor}_{L \mathbf{C}}(X, B)$ ) is a weak equivalence. The class of $\mathbf{S}$-local objects is denoted $S$ - loc, and the class of $\mathbf{S}$-colocal objects is denoted $S-$ col.

- A morphism $C \rightarrow D$ of $\mathbf{C}$ is said to be $K$-local if for any element $Y$ of $K$, the induced morphism $\operatorname{Mor}_{L \mathbf{C}}(D, Y) \rightarrow \operatorname{Mor}_{L \mathbf{C}}(C, Y)$ (resp., the induced morphism $\left.\operatorname{Mor}_{L \mathbf{C}}(Y, C) \rightarrow \operatorname{Mor}_{L \mathbf{C}}(Y, D)\right)$ is a weak equivalence. The class of $\mathbf{S}$-local morphisms is denoted $S-$ loc, and the class of $\mathbf{S}$ colocal morphisms is denoted $S-$ col.

- For $P$ a class either of objects or of morphisms of $\mathbf{M}$, I define the left hull $\operatorname{lh}(P)$ of $P$ as the class $(P-\operatorname{loc})$ - loc and the right hull $\operatorname{rh}(P)$ as the class $(P-\operatorname{col})-$ col. 
Lemma 1.5.4. If $P$ is a class either of objects or of morphisms of a $\mathrm{CMC}$ $\mathbf{M}$, then $P \subset \operatorname{lh}(P)$, and if $S$ is a class of morphisms, the left Bousfield localization $L_{S} \mathbf{M}$ is naturally isomorphic to the left Bousfield localization $L_{\mathrm{lh}(S)} \mathbf{M}$.

Dually, $P \subset \operatorname{rh}(P)$, and if $S$ is a class of morphisms, the right Bousfield localization $R_{S} \mathbf{M}$ is naturally isomorphic to the right Bousfield localization $R_{\mathrm{rh}(S)} \mathbf{M}$.

Definition 1.5.5. Suppose $\mathbf{M}$ a CMC, $\kappa$ a regular cardinal. Then a class of morphisms $S$ in $C$ is $\kappa$-sequential if $S$ is closed under colimits of $\kappa$-sequences, i.e., if for any $\kappa$-sequence of cofibrations $X_{\alpha}$ in $\mathbf{M}$, and any sequence of morphisms $X_{\alpha} \rightarrow Y$ of $S$, the morphism from the transfinite composition

$$
\operatorname{colim}_{\alpha} X_{\alpha}>Y
$$

is a morphism of $S$ as well. If the set of fibrations is $\kappa$-sequential, then one says simply that $\mathbf{M}$ has $\kappa$-sequential fibrations.

Theorem 1.5.6 (Christensen-Isaksen). If $\mathbf{M}$ is a right proper $\mathrm{CMC}$ and $K$ is a set of objects thereof such that there exists a regular cardinal $\kappa$ with the following properties :

- $\mathbf{M}$ has $\kappa$-sequential fibrations, and

- each element of $K$ is $\kappa$-small relative to the cofibrations,

then the right Bousfield localization $R_{K-\mathrm{col}} \mathbf{M}$ exists; the underlying category $i s$ the same as the underlying category of $\mathbf{M}$, and $w\left(R_{K-\mathrm{col}} \mathbf{M}\right)=K-$ col and $\operatorname{fib}\left(R_{K-\mathrm{col}} \mathbf{M}\right)=$ fibM. The cofibrant objects of $R_{K-\mathrm{col}} \mathbf{M}$ are exactly the $K-$ col-colocal objects.

About the Proof. This was proved under rather more restrictive hypotheses (right properness and cellularity) in Hirschhorn's book. The proofs there are easily translated, mutatis mutandis, to this more general setting.

Corollary 1.5.7. If $\mathbf{M}$ is a cofibrantly generated $\mathrm{CMC}$, and $K$ is a set of objects of $\mathbf{M}$, then the right Bousfield localization $R_{K-\mathrm{col}} \mathbf{M}$ exists.

Definition 1.5.8. A CMC is combinatorial if it is cofibrantly generated and presentable.

Theorem 1.5.9 (Smith). Suppose M a left proper combinatorial CMC, $S$ a set of morphisms of $\mathbf{M}$. Then the left Bousfield localization $L_{S} \mathbf{M}$ exists; the underlying category of $L_{S} \mathbf{M}$ is that of $\mathbf{M}$, and $w\left(L_{S} \mathbf{M}\right)=\ln (S)$ and $\operatorname{cof}\left(L_{S} \mathbf{M}\right)=\operatorname{cof}(\mathbf{M})$. 
About the Proof. This was proved under different hypotheses (left properness and cellularity) in Hirschhorn's book. The critical Bousfield-Smith cardinality argument carries over with only slight modification.

1.5.10. Combinatoriality is a flexible condition that is often satisfied for categories constructed from the category of simplicial sets or from that of CW complexes. For categories constructed from categories of more general topological spaces, Hirschhorn's condition of cellularity is better-behaved. Unfortunately, there are examples of left proper combinatorial CMCs that are not cellular, and there are examples of left proper cellular CMCs that are not combinatorial, so neither setting contains the other.

Theorem 1.5.11 (Lurie). Suppose $A$ is a presentable $\mathbf{S}$-category, and suppose $S$ a set of morphisms of $A$. Then the full sub-S-category $L_{S} A$ of S-local objects-i.e., objects $X$ such that for any $Y \rightarrow Z$ in $S$, the morphism $\operatorname{Mor}_{A}(Z, X) \rightarrow \operatorname{Mor}_{A}(Y, X)$ is a weak equivalence-is a reflexive subcategory of $A$.

About the Proof. This is the first major result of the overture to Lurie's thesis, rewritten for $\mathbf{S}$-categories.

Lemma 1.5.12. Suppose $\mathbf{M}$ a CMC, $S$ a set of morphisms thereof. Then $L\left(L_{S} \mathbf{M}\right)$ is equivalent to $L_{S}(L \mathbf{M})$, and this equivalence is compatible with the "localization" morphisms.

Proof. The proof is left as a nearly trivial exercise.

1.6 (The question of descent). I now formulate the central questions of descent for simplicial sets and left Quillen presheaves, and I comment upon the answers.

1.6.1. It will be convenient to recall the following key fact. Suppose $\mathbf{M}$ a cofibrantly generated closed model category, and suppose $C$ a small category. Then the functor category $\mathbf{M}^{C}$ is a cofibrantly generated closed model category in which the fibrations and weak equivalences are defined objectwise. This closed model structure on $\mathbf{M}^{C}$ will be called the projective closed model structure. Combinatoriality, left properness, and right properness are all inherited by $\mathbf{M}^{C}$ from $\mathbf{M}$.

1.6.2. The natural precursor for the theory of descent I introduce here is ordinary sheaf theory. Let $(T, \tau)$ be a site, which, for the sake of exposition, I will assume has enough points. Let $\mathbf{P S h}(T)$ be the category $\mathbf{S e t}^{T^{\mathrm{op}}}$ of presheaves (of sets) on $T$, and let $\mathbf{S h}(T, \tau)$ be the full subcategory thereof spanned by the 
sheaves. The category Set has its trivial closed model structure, and therefore $\operatorname{PSh}(T)$ has a projective closed model structure.

Theorem 1.6.3. The category $\mathbf{P S h}(T)$ has a left proper, combinatorial closed model structure in which the following conditions hold.

- Every morphism is a cofibration.

- The fibrant objects are precisely the sheaves.

- The weak equivalences are precisely those morphisms $F \rightarrow G$ such that for any point $x$ of the site $(T, \tau)$, the induced morphism $F_{x} \rightarrow G_{x}$ of sets is a bijection.

- Between any two sheaves $F$ and $G$, two morphisms $F \rightarrow G$ are homotopic iff they are equal.

Proof. Let $\operatorname{Cov}(T, t)$ be the class of morphisms $\coprod_{\alpha} U_{\alpha} \rightarrow X$ (viewed as morphisms of presheaves) for all $\tau$-covering families $\left\{U_{\alpha} \rightarrow X\right\}$ of $T$. Using the smallness of $T$, one can find easily a subset $H_{\tau}$ thereof such that the left Bousfield localization $L_{H_{\tau}} \mathbf{P S h}(T)$ of the projective closed model structure with respect to $H_{\tau}$ is the left Bousfield localization $L_{\mathbf{C o v}(T, \tau)} \mathbf{P S h}(T)$ with respect to $\operatorname{Cov}(T, \tau)$. It is immediate from the characterization of left Bousfield localizations that the listed conditions hold.

Corollary 1.6.4. The composite functor

$$
\operatorname{Sh}(T, \tau) \rightarrow \operatorname{PSh}(T) \rightarrow \operatorname{Ho} L_{\mathbf{C o v}(T, \tau)} \mathbf{P S h}(T)
$$

is an equivalence of categories.

1.6.5. The task is then to generalize this description of sheaves to stacks in $\infty$-groupoids. Since the equivalences between $\infty$-groupoids are not isomorphisms, the descent data should give not isomorphisms on overlaps, but equivalences. Additionally, the category of $\infty$-groupoids, i.e. of fibrant simplicial sets, does not itself have a closed model structure; instead, it is the class of fibrant objects within the closed model category of $\infty$-pregroupoids. For presheaves of sets, this distinction was invisible to us because every set is fibrant.

Observe that the category of presheaves of $\infty$-pregroupoids on $T$, $s \mathbf{S P r}(T)=s \mathbf{S e t}^{T^{\mathrm{op}}}$ has its projective closed model structure, which is left proper and combinatorial. The fibrant objects of this closed model structure are those presheaves of $\infty$-pregroupoids $X$ such that for any object $U$ of $T, X(U)$ is an $\infty$-groupoid. The aim now is to left-Bousfield-localize the projective model structure in a manner that is similar to the localization performed for ordinary presheaves. The weak equivalences of the resulting closed model structure should be the $\tau$-local weak equivalences, and the fibrant objects should be exactly those objects satisfying a descent condition. In 
order to guarantee that the weak equivalences are as described, it is necessary to force the fibrant objects to satsify a descent condition with respect to hypercoverings, not merely coverings. The distinction is a subtle one, as many familiar topologies have the Brown-Gersten property, which ensures that the localization with respect to coverings and with respect to hypercoverings are in fact the same.

Definition 1.6.6. A hypercovering of an object $X$ of $T$ is a simplicial presheaf $U$ along with a morphism $U \rightarrow X$ such that $U_{p}$ is a coproduct of representables for any $p>0$, and the morphism $U \rightarrow X$ is a local trivial fibration, i.e., for any object $Y$ of $T$, any $n \geqslant 0$, and any commutative square

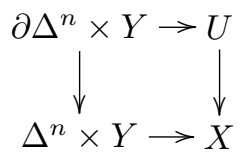

there exists a covering sieve $R$ of $Y$ such that for every $V \rightarrow Y$ in $R$, there is a lift :

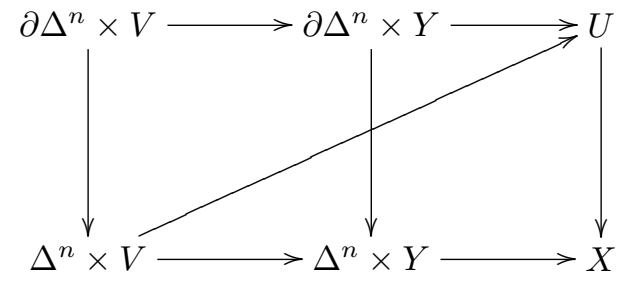

Theorem 1.6.7. There is a left proper, combinatorial closed model structure on $s \operatorname{SPr}(T)$ such that the following conditions are satisfied.

- The cofibrations are precisely the projective cofibrations.

- The fibrant objects are exactly those presheaves of $\infty$-groupoids $F$ such that for any hypercovering $U \rightarrow X$ of any object $X$ of $T$, the induced morphism

$$
F X \rightarrow \operatorname{holim}\left[\prod_{\alpha_{0}} F U_{0}^{\alpha_{0}} \rightarrow \prod_{\alpha_{1}} F U_{1}^{\alpha_{1}} \stackrel{\rightarrow}{\rightarrow} \ldots\right]
$$

is an equivalence of simplicial sets, where the $U_{n}^{\alpha_{n}}$ are the representing objects of the functors of which $U_{n}$ is the coproduct.

- The weak equivalences are exactly those morphisms $F \rightarrow G$ such that for any point $x$ of $T$, the induced morphism $F_{x} \rightarrow G_{x}$ is a weak equivalence of $\infty$-pregroupoids.

This closed model structure is called the local projective closed model structure. 
Proof. Let Hypercov $(T, \tau)$ be the class of hypercoverings of objects of $T$. Using the smallness of the site, one verifies that there exists a subset $H$ of $\operatorname{Hyper\operatorname {cov}}(T, \tau)$ such that the left Bousfield localization $L_{H_{\tau}}(s \operatorname{SPr}(T)$ of the projective closed model structure with respect to $H$ is the left Bousfield localization $L_{\mathbf{H y p e r c o v}(T, \tau)}(s \mathbf{S P r}(T)$ with respect to $\operatorname{Hypercov}(T, \tau)$. The listed properties follow immediately from the characterization of left Bousfield localizations.

Definition 1.6.8. An $\infty$-stack or stack in $\infty$-groupoids is a fibrant object of the local projective closed model category of presheaves of $\infty$-pregroupoids.

1.6.9. Now it is possible to ask the question : is the algebraic $K$-theory functor $K$-a presheaf of $\infty$-groupoids - an $\infty$-stack for the étale topology? for any other interesting topologies?

The answer turns out to be a very qualified yes. First of all, Thomason showed that algebraic $K$-theory does satisfy Zariski descent. For smooth schemes, he also demonstrated that $K$-theory satisfies Nisnevich descent. Over a field with resolution of singularities, Haesemeyer showed that homotopy $K$-theory satisfies cdh descent. Finally, $K$-theory does not itself satisfy étale descent, but its failure to do so is in a sense bounded : Thomason showed that Bott-inverted $K$-theory satisfies descent.

This is still not quite enough to prove the Beilinson-Lichtenbaum conjecture. The Beilinson-Lichtenbaum spectral sequence does not converge below the line $p+q=d$, where $d$ is the cohomological dimension of the absolute Galois group of the ground field. It therefore cannot be a descent spectral sequence for $K$-theory as a presheaf of $\infty$-groupoids alone. The central idea, due to Gunnar Carlsson, is to view $K$ instead as a presheaf of ring objects in a certain category of Mackey functors. This approach, while promising, seems not to have generated much attention, despite the fact that it may very well lead toward a conceptual proof of the Beilinson-Lichtenbaum conjecture. The details of the set-up are perhaps better left for another day.

1.6.10. To study our second question, namely, whether the left Quillen presheaf Cplx satisfies descent, one may be tempted to try to use our work on stacks in $\infty$-groupoids to design a notion of $(\infty, 1)$-stacks. In particular, one might study presheaves of $\mathbf{S}-$ categories on $T$. The category $(\mathbf{S}-\mathbf{C a t})^{T^{\mathrm{op}}}$ of all such presheaves on $T$ has its projective closed model structure, and one can try to take the left Bousfield localization of this projective closed model structure with respect to all hypercoverings. The fibrant objects of the resulting closed model category would be the presheaves of $\mathbf{S}$-categories that satsify descent, and the weak equivalences would be the local $\mathbf{S}$-equivalences. 
Unfortunately, this seems to require an ability to view an $\infty$-groupoid $G$ as an $\mathbf{S}$-category $X_{G}$ in such a way that the objects of $X_{G}$ are precisely those of $G$ and the $n$-morphisms of $X_{G}$ are precisely those of $G$. This is impossible, because the 1-morphisms of $\infty$-groupoids are only composable up to a 2 -morphism, whereas the 1-morphisms of $\mathbf{S}$-categories are strictly composable. It is therefore unclear how to view hypercoverings as $\mathbf{S}$-functors. In order to continue, therefore, it is necessary to design a theory of $(\infty, 1)$-categories wherein the 1 -morphisms are only composable up to a 2 -morphism.

\section{Delooping machines and $(\infty, n)$-categories}

Maybe at times I like to give the impression, to myself and hence to others, that I am the easy learner of things in life, wholly relaxed, "cool" and all that-just keen for learning, for eating the meal and welcome smilingly whatever comes with it's message, frustration and sorrow and destructiveness and the softer dishes alike. This of course is just humbug, an image d'Epinal which at whiles I'll kid myself into believing I am like. Truth is that I am a hard learner, maybe as hard and as reluctant as anyone.-A. Grothendieck.

\section{1 (Infinite loop spaces and delooping machines)}

I will give what I hope is convincing evidence that the study of infinite loop spaces in algebraic topology has the support of a powerful and deep theory, and that this theory has pleasant formal properties.

I will begin this subsection by discussing loop spaces, giving their nice properties, and giving a few examples. Then I will introduce the Stasheff associahedra, $A_{\infty}$-spaces, infinite loop spaces, and finally Segal's theory of delooping machines. It will be seen that an infinite hierarchy of higher homotopies must be dealt with, and it will also be seen that there are two ways of managing this hierarchy : the Stasheff, or operadic, method, which deals more or less explicitly with the combinatorics of the homotopies, and the Segal, or simplicial, method, which hides the combinatorics behind the theory of simplicial sets. One can today look at these simple constructions through more sophisticated eyes, and this is exactly what I will do here.

2.1.1. To fix ideas, I will work in the category $\mathscr{T}$ of pointed, compactly generated spaces (with basepoint-preserving continuous maps). Spaces in $\mathscr{T}$ are those spaces $X$ that have the following pleasant properties :

- $X$ is weakly Hausdorff ; that is, the image of any continuous map from a compact Hausdorff space to $X$ is closed in $X$. 
- $X$ is Kelley; that is, any subset $U$ of $X$ that is compactly open -in the sense that $U$ has open inverse image under any continuous map from a compact Hausdorff space to $X$-is in fact open.

The category $\mathscr{T}$ itself has extremely nice properties :

- Any space $Y$ can be made into a Kelley space $k Y$ (with the same underlying point set) by declaring that a subset $U$ is open in $k Y$ iff $U$ is compactly open in $Y$. It can then be made into a compactly generated space $w Y$ by taking the maximal weak Hausdorff quotient. This defines a functor $w$ from the category of all pointed spaces to $\mathscr{T}$.

- $\mathscr{T}$ has all small limits and colimits, which are given by taking the limits and colimits in the category of all topological spaces and then applying $w$. As a rule, I will remove $w$ from the notation, as quite often it is not even necessary to apply it.

- Though the product in $\mathscr{T}$ is the product $-\times-$, where the basepoint is given by the point whose coordinates are the basepoints of the factors, there is also a kind of "tensor product" given by the smash product $-\wedge-$. $\mathscr{T}$ is closed monoidal category with this product, in the sense that applying $w$ to a set of continuous, basepoint-preserving maps with the compactopen topology gives an internal Mor functor (where the basepoint is the constant map) so that $\operatorname{Mor}(X \wedge Y, Z)=\operatorname{Mor}(X, \underline{\operatorname{Mor}}(Y, Z)$.

- The geometric realization of any pointed simplicial set is an object of $\mathscr{T}$; this defines a functor $|-|$ from the category $\mathscr{S}$ of pointed simplicial sets to $\mathscr{T}$.

- The geometric realization functor is left adjoint to the functor that sends a compactly generated space to its total singular complex. This pair forms a Quillen equivalence between $\mathscr{S}$ and $\mathscr{T}$ with their usual model structures (where in particular the weak equivalences are given by maps that induce isomorphisms on all homotopy groups), and so these functors descend to quasi-inverses between the homotopy categories Ho $\mathscr{S}$ and Ho $\mathscr{T}$.

When I write the word 'space,' I mean an object of $\mathscr{T}$, and when I write 'map,' I mean a continuous, basepoint-preserving map, unless otherwise noted.

$\mathscr{T}$ is really the largest category of pointed spaces that is so well-behaved formally.

2.1.2. With the conventions above, the loop space $\Omega X$ of a space $X \in \mathscr{T}$ is the space $\underline{\operatorname{Mor}}\left(S^{1}, X\right)$, where of course $S^{1}$ is a pointed circle. The loop space can be thought of as a flabby version of the Poincaré fundamental group of $X$; it comes with higher homotopies. Indeed, the connected components of $\Omega X$ form 
a group, and that group is naturally isomorphic to $\pi_{1} X$. More generally, one can check easily that $\pi_{i}(\Omega X)$ is naturally isomorphic to $\pi_{i+1} X$ for any $i \geqslant 0$.

Everyone who has seen the fundamental group defined knows that $\Omega X$ is not quite a topological group, even though its connected components are. One can compose any two loops by running through one and then the other, each at double speed. More precisely, the pinching map $S^{1} \rightarrow S^{1} \vee S^{1}$ given by the identification of a non-basepoint with the basepoint induces a natural transformation from $\underline{\operatorname{Mor}}\left(S^{1} \vee S^{1},-\right)$ to $\underline{\operatorname{Mor}}\left(S^{1},-\right)$, so that there is a map $\Omega X \times \Omega X \rightarrow \Omega X$, which we can call $\mu$. But the constant loop, which is the basepoint $\star$ of $\Omega X$, is not a strict unit for this mutliplication law : neither $\mu(\gamma, \star)$ nor $\mu(\star, \gamma)$ is equal to $\gamma$, since, for example, $\mu(\gamma, \star)$ is the loop that dawdles at the basepoint for half the time around the circle, and then rushes through $\gamma$ at double speed. Nor is this multiplication law is strictly associative : given three loops $\alpha, \beta$, and $\gamma$, the loop $\mu(\alpha, \mu(\beta, \gamma))$ runs through $\gamma$ and $\beta$ both at quadruple speed and then runs through $\alpha$ at half speed, whereas $\mu(\mu(\alpha, \beta), \gamma)$ runs through $\gamma$ at half speed and then runs through $\beta$ and $\alpha$ at quadruple speed. (One can actually tidy up this part of the situation by looking at what are called "Moore loops" to make $\Omega X$ equivalent to a topological monoid, but I needn't go into this here.) Finally, note that the inverse loop is not a strict inverse.

The structure of $\Omega X$ is described by saying that $\mu$ gives $\Omega X$ the structure of a group in the homotopy category of spaces. That is, one has the following : - The basepoint is a homotopy unit. If ' $\star$ ' denotes the inclusion of the basepoint into $\Omega X$, then $\mu \circ(\star \times \mathbf{1})$ and $\mu \circ(\mathbf{1} \times \star)$ are both homotopic to the identity on $\Omega X$.

- The multiplication is homotopy-associative. $\mu \circ(\mu \times \mathbf{1})$ and $\mu \circ(\mathbf{1} \times \mu)$ are homotopic maps from $\Omega X \times \Omega X \times \Omega X$ to $\Omega X$.

- There are homotopy inverses. If ' $\nu$ ' denotes the self-map on $\Omega X$ that sends a loop to its inverse, and $\Delta$ is the diagonal map $\Omega X \rightarrow \Omega X \times \Omega X$, then $\mu \circ(\mathbf{1} \times \nu) \circ \Delta$ and $\mu \circ(\nu \times \mathbf{1}) \circ \Delta$ are each homotopic to the map that factors through $\star$.

Serre called a space satisfying the first and second of these of these a homotopyassociative $H$-space, in honor of Hopf. We will, by an abuse of terminology, use the somewhat handier expression $H$-space to refer to such objects, which, morally, are spaces $Z$ with maps $Z \times Z \rightarrow Z$ that give monoids in the homotopy category. The third condition might seem like a key fact about loop spaces, but in fact it is something of a red herring : it can be done away with altogether by looking more closely at the first two conditions, which is precisely what I will do. 
Serre used the loop space quite well : he remarked that if one took the usual unit interval $I$ with basepoint at (say) 0 , one could design a continuous map from the space $\Pi X=\underline{\operatorname{Mor}}(I, X)$ of paths in $X$ to $X$ just by evaluation at 1. This map is a very good map; to be precise, it is a fibration in the sense of Serre. The fibre over the basepoint of $X$ is the loop space $\Omega X$. Moreover $\Pi X$ is contractible : one can write the contraction explicitly as shrinking along the paths. These facts indicate the possibility of an analogy with the universal principal $G$-bundle over the classifying space B $G$ of a topological group $G$ : morally, $X$ can be thought of as the classifying space of the $H$-space $\Omega X$, and $\Pi X$ can be thought of as the universal torsor for $\Omega X$. From this point of view, $\Omega X$ is the more primitive object, and $X$ is built from it.

Of course the endofunctor $\Omega=\underline{\operatorname{Mor}}\left(S^{1},-\right)$ has, by closedness, a left adjoint $\Sigma=-\wedge S^{1}$, called suspension. This means that for any $X$ there are maps $X \rightarrow \Omega \Sigma X$ (the unit) and $\Sigma \Omega X \rightarrow X$ (the counit) such that the induced maps $\Sigma X \rightarrow \Sigma \Omega \Sigma X$ and $\Omega \Sigma \Omega X \rightarrow \Omega X$ are left- and right-invertible, respectively, with the obvious inverses.

Example 2.1.3. Obviously, it does not take a lot of effort to find examples of loop spaces. Here are some spaces that have the homotopy type of loop spaces :

— Z $\mathbf{Z}$, viewed as a discrete topological group with basepoint 0 , is homotopy equivalent to the loop space of $S^{1}$ : each of the connected components of $\Omega S^{1}$ is contractible!

- $S^{1}$ has the homotopy type of the loop space of $\mathbf{P}_{\mathbf{C}}^{\infty}$.

- Generalizing the above two examples, any Eilenberg-Mac Lane complex $K(\pi, n)$ has the homotopy type of $\Omega K(\pi, n+1)$. To check this, just note that the Eilenberg-Mac Lane complexes are CW complexes, and that there is a weak equivalence $K(\pi, n) \rightarrow \Omega K(\pi, n+1)$, which therefore must be a homotopy equivalence.

- Any topological group that has the homotopy type of a CW complex has the homotopy type of a loop space. Suppose $G$ is such a group, and construct its classifying space $\mathrm{B} G$ as a $\mathrm{CW}$ complex. The universal $G^{-}$ torsor is then a contractible space $E G$ along with a principle $G$-bundle $E G \rightarrow \mathrm{B} G$ that is universal in the sense that for any space $X$, the pullback map from $\operatorname{Mor}(X, \mathrm{~B} G)$ to the space of principle $G$-bundles over $X$ is a bijection. In particular, the long exact sequence of a fibration tells us that $G$ has the homotopy type of $\Omega B G$. So in the case where $X$ is a space that already has the homotopy type of a classifying space, $X$ really is the classifying space of $\Omega X$, and $\Pi X$ really is the universal torsor for $\Omega X$. 
Exercise 2.1.4. If one adopts the attitude that loop spaces are more important than regular spaces, one can go into the business of sniffing out loop spaces. The discussion above indicates that a good place to begin is with $H$ spaces. But this is not the end of the story : give an example of an $H$-space (in the sense described above) that is not a loop space. (Hint : By 2.1.3, you know already that such an $H$-space will not be a topological group. After you have found a $H$-space that you think is not a loop space, you can show that your space fails to satisfy the adjunction property of 2.1.2.) You can even construct such an $H$-space by imposing a new multiplication law on a CW complex with the homotopy type of a topological group! So here is a place where you cannot rely on the ordinary homotopy category to do the work for you.

2.1.5. If one wishes to find an intrinsic characterization of loop spaces, one can start by looking at $H$-spaces. The exercise above indicates that loop spaces are not run-of-the-mill $H$-spaces. Looking more deeply at the problem, we can see that there is a little ambiguity in the axioms for an $H$-space. An $H$-space must have a multiplication law that gives a monoid in the homotopy category, but without any restrictions on the homotopies themselves. In particular, let us have a look at the homotopy associativity axiom : fix an $H$-space $X$ and a homotopy $H$ from $\mu \circ(\mu \times \mathbf{1})$ to $\mu \circ(\mathbf{1} \times \mu)$. We can easily think of five maps $(\Omega X)^{\times 4}=\Omega X \times \Omega X \times \Omega X \times \Omega X \rightarrow \Omega X$, which we can arrange as vertices of a pentagon :

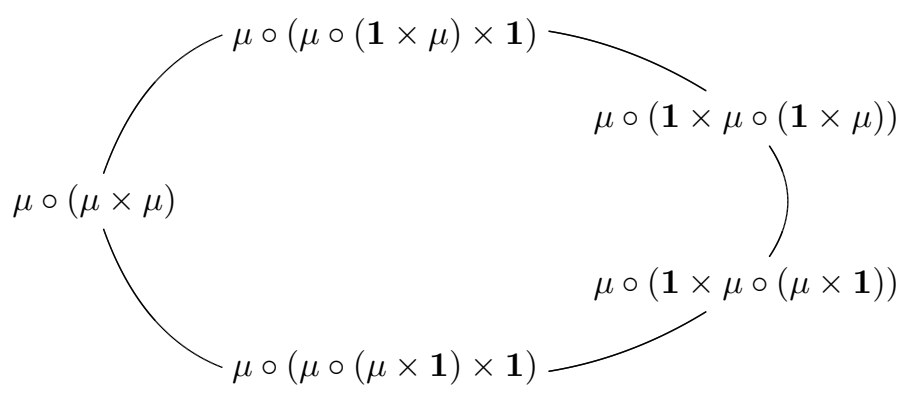


This picture corresponds to the five ways of bracketing four loops $\alpha, \beta, \gamma, \delta \in$ $\Omega X$ in order :

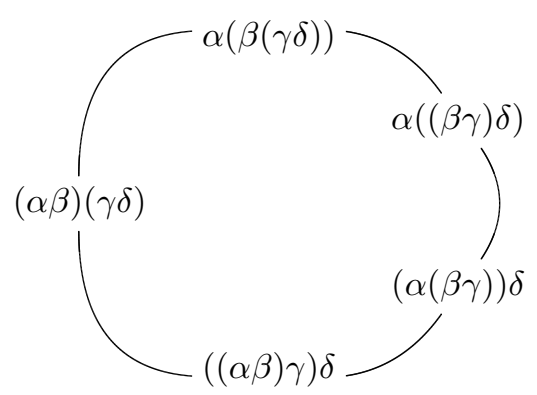

The edges of this pentagon in fact refer to homotopies :

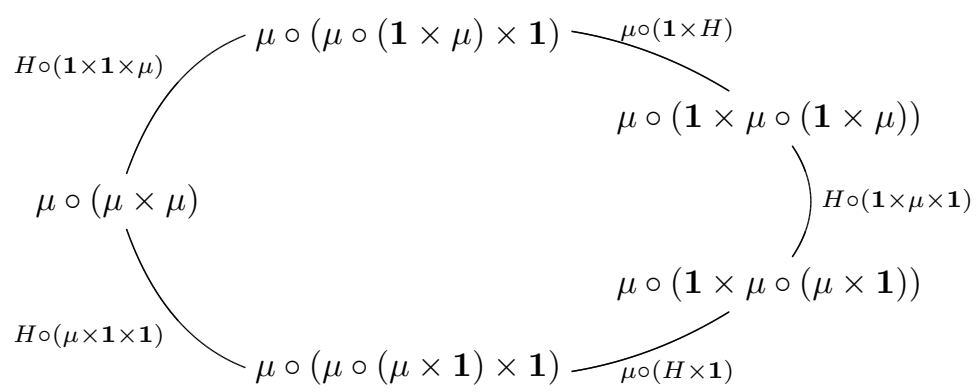

The shape of the diagram is no accident. By pasting these homotopies together, we build a map $S^{1} \times(\Omega X)^{\times 4} \rightarrow \Omega X$. But now a moment's reflection is all that is required to see that these homotopies commute, in the sense that the corresponding map $S^{1} \rightarrow \underline{\operatorname{Mor}}\left((\Omega X)^{\times 4}, \Omega X\right)$ (which need not respect basepoints, and so is not really a map in our category) is actually null-homotopic ; 
here is the picture :

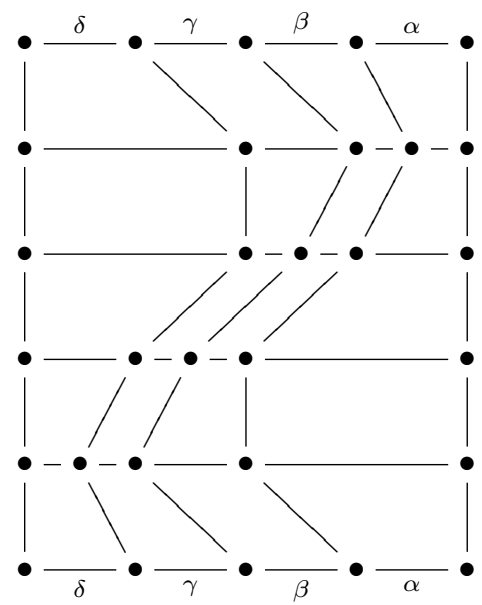

with the top and bottom edges identified, of course. Graphically, one just straightens the vertical paths out in a continuous way. In other words, the map $S^{1} \times(\Omega X)^{\times 4} \rightarrow \Omega X$ can be extended to a map $e^{2} \times(\Omega X)^{\times 4} \rightarrow \Omega X$, where $e^{2}$ is a 2 -cell (or disk).

This need not have happened; nothing in the axioms of an $H$-spaces forces this null-homotopy to exist. But for loop spaces, we have this condition, which one can think of as a secondary homotopy condition. One can carry on like this, obtaining a tertiary homotopy condition, etc.

2.1.6. Stasheff, inspired by Sugawara's earlier recognition principle for loop spaces, defined spaces $K_{m}$ for each $m>1$, called the associahedra or the Stasheff polytopes, as $(m-2)$-cells $e^{m-2}$ with a prescribed subdivision of the boundary $S^{m-3}$ into a polyhedron with $b_{m}$ vertices, where $b_{m}$ is the number of ways to form pairwise brackets about $m$ letters in a fixed order. So $K_{2}$ is a point and $K_{3}$ is a unit interval; not much work can be done at the boundary. Then he defined $A_{m}$-spaces for every $m>1$ inductively as follows : an $A_{2}$-space is simply an $H$-space $Z$; from the map

$$
K_{2} \times Z^{\times 2}=Z^{\times 2} \stackrel{M_{2}}{\longrightarrow} Z
$$

one constructs a map

$$
\left(\partial K_{3}\right) \times Z^{\times 3}=S^{0} \times Z^{\times 3} \stackrel{\partial M_{3}}{\longrightarrow} Z
$$

by sending $(0, \lambda, \mu, \nu)$ to $M_{2}\left(M_{2}(\lambda, \mu), \nu\right)$ and sending $(1, \lambda, \mu, \nu)$ to $M_{2}\left(\lambda, M_{2}(\mu, \nu)\right)$. 
Now an $A_{m}$-space is an $A_{m-1}$-space $Z$ such that the map

$$
\left(\partial K_{m}\right) \times Z \times m \stackrel{\partial M_{m}}{\longrightarrow} Z
$$

constructed (in a completely combinatorial way, the general description of which is unenlightening) from the maps $Z^{\times n} \stackrel{M_{n}}{\longrightarrow} Z$ for $n<m$, extends to a map

$$
K_{m} \times Z^{\times m} \stackrel{M_{m}}{\longrightarrow} Z
$$

Of course an $A_{\infty}$-space is a space $Z$ with maps

$$
K_{m} \times Z^{\times m} \stackrel{M_{m}}{\longrightarrow} Z
$$

for all $m>1$ which give $X$ the structure of an $A_{n}$-space for any $n>1$.

Exercise 2.1.7. Find an explicit formula for $b_{m}$ in terms of $m$. Can you say how many faces (i.e., $(m-3)$-cells $\partial K_{m}$ must have?

2.1.8. $K_{2}$ is, as we have already said, a point, and $K_{3}$ is an interval. $K_{4}$ is the solid Stasheff pentagon drawn above, and $K_{5}$ is a solid polyhedron with 14 vertices, 21 edges, and 9 faces ( 3 quadrilaterals and 6 pentagons). A nice rotatable graphic of $K_{5}$ is available from http ://www.labmath.uqam.ca/ chapoton/stasheff.html.

Theorem 2.1.9 (Stasheff). An $H$-space $Z$ is a loop space iff $Z$ is an $A_{\infty}$ space and $\pi_{0} Z$ is a group. Moreover $\Omega$ induces an equivalence of categories between the category of connected spaces and the category of $A_{\infty}$-spaces whose connected components form a group.

About the Proof. The essence of the idea is to construct and use a classifying space and universal torsor for an $A_{\infty}$-space, following the intuition suggested above.

2.1.10. Stasheff's associahedra combine to form what is called an operad, a notion due essentially to Boardman, Vogt, and May. I'll briefly describe this notion in a heuristic way. Operads can live in any symmetric monoidal category, but there is no reason to use that level of generality ; I'll stick to my category $\mathscr{T}$. An operad $\mathscr{X}$ here consists of objects $X_{j}$ for $j \geqslant 0$, an action of the symmetric group $\Sigma_{j}$ on each $X_{j}$, and maps from $X_{j_{1}} \times \cdots \times X_{j_{n}}$ to $X_{j_{1}+\cdots+j_{n}}$ which are associative, unital, and equivariant in a precise sense, which I'll not go into here.

It's much better to see an example of an operad in action. For some $n>0$, one has the little $n$-cubes operad $\mathscr{P}(n)$, which acts on $n$-fold loop spaces, i.e., 
spaces of the form $\Omega^{n} X$ for some space $X$. To begin, build a subspace $P_{j}(n) \subseteq$ $\underline{\operatorname{Mor}}\left(S^{n}, \bigvee_{m=1}^{m=j} S^{n}\right)$ by only considering those maps from $S^{n}=I^{n} / \partial I^{n}$ to the bouquet of $m$ copies of $S^{n}$ that lift to maps that are constant outside $m$ cubes $C_{i}$ in $I^{n}$, each of which is mapped linearly onto the $i$-th copy of $I^{n} . \Sigma_{j}$ acts on $P_{j}(n)$ in a natural way, and one can check that the $P_{j}(n)$ form an operad $\mathscr{P}(n)$.

Now we can see that $\mathscr{P}(n)$ acts on $\Omega X$ : each point

$$
S^{n} \rightarrow \bigvee_{m=1}^{m=j} S^{n}
$$

of $P_{j}(n)$ induces a map

$$
\left(\Omega^{n} X\right)^{\times j}=\underline{\operatorname{Mor}}\left(\bigvee_{m=1}^{m=j} S^{n}, X\right) \rightarrow \underline{\operatorname{Mor}}\left(S^{n}, X\right)=\Omega^{n} X
$$

and we have our action.

An $E_{n}$-space is a space along with an action of $\mathscr{P}(n)$. So $n$-fold loop spaces are $E_{n}$-spaces. J. P. May proved the analogue to 2.1.9, which essentially says that:

- $\Omega^{n}$, viewed as a functor to $E_{n}$-spaces whose connected components form a group, has a left adjoint $\mathrm{B}^{n}$,

- that the components $X \rightarrow \Omega^{n} \mathrm{~B}^{n} X$ of the unit are equivalences of $E_{n}$ spaces whenever $\pi_{0} X$ is a group, and

- that the components $\mathrm{B}^{n} \Omega^{n} Z \rightarrow Z$ of the counit are homotopy equivalences whenever $Z$ is $(n-1)$-connected.

Example 2.1.11. Further still, one can try to give a recognition principle for infinite loop spaces, i.e., spaces of the form $\Omega^{\infty} X=$ colim $\Omega^{m} X$. This is indeed possible, but before we discuss this, we should remark on some interesting infinite loop spaces :

- Eilenberg-Mac Lane spaces are always infinite loop spaces, since $K(\pi, n)$ always has the homotopy type of $\Omega K(\pi, n+1)$.

- Bott Periodicity states that $\mathbf{Z} \times \mathrm{B} U$ has the homotopy type of its double loop space $\Omega^{2}(\mathbf{Z} \times \mathrm{B} U)$, and that $\mathbf{Z} \times \mathrm{B} O$ has the homotopy type of its 8 -fold loop space $\Omega^{8}(\mathbf{Z} \times \mathrm{BO})$. Hence both $\mathbf{Z} \times \mathrm{B} U$ and $\mathbf{Z} \times \mathrm{BO}$ are infinite loop spaces.

- The classifying space of a category $\mathscr{C}$ is a space assigned to $\mathscr{C}$ : the classifying space of $\mathscr{C}$ is then the geometric realization $\mathrm{B} \mathscr{C}=|\nu \cdot \mathscr{C}|$. The classifying space of a symmetric monoidal category is always an infinite loop space.

2.1.12. Note that the little $(n-1)$-cubes operad embeds into the little $n$ cubes operad. Then the little cubes operad $\mathscr{P}(\infty)$ is the colimit of the $\mathscr{P}(n)$ for all $n$. An $E_{\infty}$-space is a space equipped with an action of $\mathscr{P}(\infty)$. It clearly 
acts on an infinite loop space $\Omega^{\infty} X$, and now one can prove an analogue of 2.1.9 for infinite loop spaces, which essentially says that a space is an infinite loop space iff it is an $E_{\infty}$-space.

2.1.13. I can try an alternative strategy for understanding loop spaces and infinite loop spaces that is a little more accessible : I can try to talk simplicial sets into keeping track of classes of formulas like $\mu \circ(\mu \times \mathbf{1})$ and $\mu \circ(\mathbf{1} \times \mu)$ for me. This is Segal's approach to loop spaces and infinite loop spaces, introduced through a privately-distributed manuscript called "Homotopy-Everything $H$-Spaces," sketched by Anderson, and continued in Segal's famous paper, "Categories and Cohomology Theories." If $Z$ is an $H$-space, then one can take a variant of the above construction by viewing $Z$ as a monoid in the homotopy category, which is in particular a category $\mathscr{Z}$, and we can then take the nerve $\nu_{\bullet} \mathscr{Z}$. This can be viewed as a simplicial object in Ho $\mathscr{T}$ with the property that $[n]$ is sent to $Z^{\times n}$, the first degeneracy map is the multiplication map $\mu$, and the rest of the face and degeneracy maps are given by obvious products of $\mu$, $\mathbf{1}$, and inclusions. If one finds a lift of this to a simplicial object in $\mathscr{T}$, one can form a classifying space, and proceed using the yoga of Stasheff and Sugawara.

Suppose $\Lambda_{\bullet}$ a simplicial object of $\mathscr{T}$. Then one has $n$ face maps $\Lambda_{n} \rightarrow \Lambda_{1}$, which can be combined to form a single map $\Lambda_{n}>\left(\Lambda_{1}\right)^{\times n}$. Segal calls $\Lambda_{\bullet}$ a special simplicial space if these maps are homotopy equivalences for every $n \geqslant 1$.

This is clearly a very elegant way to organize arbitrarily high homotopies, and it is a point of view that informs a large part of the applications to algebraic geometry that we will encounter here.

Theorem 2.1.14 (Segal). A space $Z$ is an $A_{\infty}$-space iff there exists a special simplicial object $\Lambda_{\bullet}$ in $\mathscr{T}$ such that $\Lambda_{1}=Z$.

2.1.15. In this situation, $\Lambda_{n}$ is homotopy equivalent to $Z^{\times n}$, and $\Lambda_{n}$ is playing the role of the product $P_{j} \times Z^{\times n}$. Segal's approach is in some sense doubly nice : not only is it refreshingly easy to describe the parameter spaces (especially since the $\Lambda_{n}$ are pulling double duty!), but the ugly combinatorics are hidden behind the pleasant opaque veneer of simplicial sets.

This same trick will work for infinite loop spaces, but one needs a bigger category. Define $\Gamma$ to be the category whose objects are finite sets, and whose morphisms are maps between the power sets of these finite sets that preserve disjoint unions. A $\Gamma$-space is then a contravariant functor from $\Gamma$ to $\mathscr{T}$. Now $\Delta$ embeds into $\Gamma$ in an obvious way : any nondecreasing map $f$ from $\{0,1, \ldots, m\}$ to $\{0,1, \ldots, n\}$ gives a morphism from the power set of $\{0,1, \ldots, m\}$ to that of $\{0,1, \ldots, n\}$ by sending any one-point set $\{i\}$ to the set $\{0 \leqslant j \leqslant n \mid f(i-1)<$ 
$j \leqslant f(i)\}$. Hence a $\Gamma$-space gives rise to a simplicial space. Now a special $\Gamma$-space is a $\Gamma$-space that gives rise to a special simplicial space in this way.

Theorem 2.1.16 (Segal's Delooping Machine). A space $Z$ is an $E_{\infty}-$ space iff there exists a special $\Gamma$-space $P$ such that $P(\star)=Z$.

Exercise 2.1.17. The Segal approach is so elegant that I can without hesitation give the following exercise : read Stasheff's proof of 2.1.9, and use this strategy to devise a proof of the theorems of Segal.

2.1.18. I've given two ways of dealing with the hierarchies of higher homotopies in the associativity of the product of loops in loop spaces and infinite loop spaces. Segal's delooping machine is certainly much easier to define and prove theorems with, but it has the unfortunate disadvantage of not being very explicit. $\Gamma$-spaces are very flabby objects, and explicit computations could be very difficult. Nevertheless, for our applications, Segal's theory has the nicest formal properties, and his delooping machine will be used heavily (sometimes in disguise) in the sequel.

2.2 (Weak $(\infty, n)$-categories). Beginning with the theory of $\infty$ pregroupoids and $\infty$-groupoids, I now use Segal's delooping machine to produce a theory of $(\infty, n)$-categories. The idea is that an $(\infty, 1)$-category is a multi-object version of a Segal space, i.e., $(\infty, 1)$-categories are to Segal spaces what categories are to monoids.

Presumably, any delooping machine can be used, in an altogether analogous way, to produce a theory of $(\infty, n)$-categories that, by the May-Thomason Uniqueness Theorem, is equivalent (in an appropriate sense) to the one I introduce here. This idea has not yet been made precise, but Toën's work on an axiomatization of the theory of $(\infty, 1)$-categories inspires hope.

Definition 2.2.1. I define Segal $n$-precategories recursively : suppose that one has defined the following :

- a category Se - $(n-1)$ - PC of Segal $(n-1)$-precategories, and

- a fully faithful functor $c_{n-1}$ from Set to $\mathbf{S e}-(n-1)-\mathbf{P C}$, the objects of the essential image of which are called constant.

Then I define the following :

- the category $\mathbf{S e}-n-\mathbf{P C}$ of Segal $n$-precategories, the full subcategory of the category $\operatorname{Mor}\left(\Delta^{\mathrm{op}}, \mathbf{S e}-(n-1)-\mathbf{P C}\right)$ of simplicial objects in the category of Segal $(n-1)$-precategories spanned by those simplicial objects $[p] \mapsto A_{p}$ such that $A_{0}$ is a constant Segal $(n-1)$-precategory, and 
- the fully faithful functor $c_{n}$ from Set to Se - $(n-1)-\mathbf{P C}$, defined by sending a set $S$ to the constant simplicial object taking its value at $c_{n-1}(S)$, so that a constant Segal $n$-category is a constant simplicial object of $\mathbf{S e}-(n-1)-\mathbf{P C}$, taking its value on a constant Segal $(n-1)-$ precategory.

2.2.2. One can "dévisser" this definition : a Segal $n$-precategory is nothing more than a multisimplicial set

$$
\begin{gathered}
\left(\Delta^{\mathrm{op}}\right)^{\times(n+1)} \longrightarrow \text { Set } \\
\left(m_{1}, \ldots, m_{n+1}\right) \mapsto A_{\left(m_{1}, \ldots, m_{n+1}\right)}
\end{gathered}
$$

that satisfies Tamsamani's constancy condition, i.e., for any $\left(m_{1}, \ldots, m_{i}\right) \in$ $\left(\Delta^{\mathrm{op}}\right)^{\times i}$, if $m_{i}=0$, then the functor $\left(m_{i+1}, \ldots, m_{n+1}\right) \mapsto A_{\left(m_{1}, \ldots, m_{n+1}\right)}$ is a constant functor $\left(\Delta^{\mathrm{op}}\right)^{\times(n-i+1)} \rightarrow$ Set .

Hence, forming the quotient $\Theta^{n+1}$ of $\left(\Delta^{\mathrm{op}}\right)^{\times(n+1)}$ by identifying

$$
\left(m_{1}, \ldots, m_{n+1}\right)=\left(m_{1}, \ldots, m_{i-1}, 0, \ldots, 0\right),
$$

whenever $m_{i}=0$, then one can define a Segal $n$-precategory as a functor from $\Theta^{n+1}$ to Set.

2.2.3. Now to define Segal $n$-categories. Suppose, for the sake of discussion, that $A$ is a Segal 1 -precategory. One should expect $A$ to be a Segal 1 -category provided that $A$ is a good model for an $\infty$-category, all of whose $n$-morphisms are invertible if $n>1$. If $x$ and $y$ are elements of the set $A_{0}$, then one should probably expect that one can define the simplicial set of morphisms from $x$ to $y$. The two face maps from [0] to [1] in $\Delta$ induce a morphism from $A_{1}$ to $A_{0} \times A_{0}$ by taking the product. One should think of this morphism as sending a morphism to the pair consisting of its source and target. So a composition law should resemble a morphism from $A_{1} \times{ }_{A_{0}} A_{1}$ to $A_{1}$, where the morphisms from $A_{1}$ to $A_{0}$ consist of the morphism from $A_{1}$ to $A_{0} \times A_{0}$, composed with the first and second projection, respectively. This not quite what I have. I have the obvious variant of the Segal map from 2.1.13: $A_{p} \rightarrow A_{1} \times{ }_{A_{0}} \cdots \times \times_{A_{0}} A_{1}$ (where the maps alternate : source, target, source, target, ...), and I have the obvious map $A_{p} \rightarrow A_{1}$ (induced by sending [1] to [p] via the inclusion of $\{0, p\}$ into $[p])$. Instead of defining a strict map from $A_{1} \times{ }_{A_{0}} \cdots \times_{A_{0}} A_{1}$ to $A_{1}$ making the diagram commute, one requires instead that the Segal map is a weak equivalence of simplicial sets. Hence composition is only defined up to homotopy. 
Definition 2.2.4. I define Segal $n$-categories recursively : let the category Se-0-Cat of Segal 0-categories be the category $\mathbf{S e - 0 - P C ~ o f ~ s i m p l i c i a l ~ s e t s . ~}$ For any Segal 0 -category $A$, let $\tau_{\leqslant 0} A$ be the discrete category whose objects are $\pi_{0}(A)$ (i.e., the connected components of $A$ ). Now suppose that one has defined the following:

- a full subcategory Se - $(n-1)$ - Cat of Se - $(n-1)-\mathbf{P C}$, whose objects are called Segal $(n-1)$-categories,

- for any object $A \in \mathbf{S e}-(n-1)$ - Cat, a category $\tau_{\leqslant 0} A$, called the homotopy category of $A$,

- a subcategory of $\mathbf{S e}-(n-1)$ - Cat, whose objects are the same and whose morphisms are called equivalences.

Then I define the following :

- the category Se - $n$ - Cat of Segal n-categories, the full subcategory of Se $-n-\mathbf{P C}$ spanned by those Segal $n$-precategories $A$ such that for any $[p] \in \Delta^{\mathrm{op}}, A_{p}$ is an $(n-1)$-category, and the Segal morphism $A_{p} \rightarrow A_{1} \times{ }_{A_{0}} \cdots \times{ }_{A_{0}} A_{1}$ is an equivalence of $(n-1)$-categories,

- for any object $A \in \mathbf{S e}-n$ - Cat, the homotopy category $\tau_{\leqslant 0} A$ is the category whose set of objects is $A_{0}$ and whose set of morphisms is the set of isomorphism classes of objects of $\tau_{\leqslant 0} A_{1}$ (with the obvious sources and targets)

- a morphism $A \rightarrow B$ of Segal $n$-categories is a Dwyer-Kan equivalence iff it induces a Dwyer-Kan equivalence of categories $\mathrm{Ho} A \rightarrow \mathrm{Ho} B$ and, for any $[p] \in \Delta^{\mathrm{op}}$, an equivalence of Segal $(n-1)$-categories $A_{p} \rightarrow B_{p}$.

Exercise 2.2.5. Show that Ho $A$ is actually a category; that is, show that compositions exist, and composition is associative.

2.2.6. One has now a kind of strictification endofunctor SeCat of $\mathbf{S e}-n-$ PC, along with a natural transformation $\sigma$ from the identity functor to SeCat such that :

- for any Segal $n$-precategory $A, \operatorname{SeCat}(A)$ is a Segal $n$-category;

- for any Segal $n$-precategory $A, \sigma_{A}$ induces a bijection between $A_{0}$ and $\operatorname{SeCat}(A)_{0}$;

- for any Segal $n$-category $A, \sigma_{A}$ is a Dwyer-Kan equivalence of $n$ categories; and

- for any Segal $n$-precategory $A, \operatorname{SeCat}\left(\sigma_{A}\right)$ is a Dwyer-Kan equivalence of $n$-categories.

2.2.7. Though it would take me somewhat far afield to define them, the theory of Segal $n$-categories is sufficiently rich to deal with the usual universal constructions in category theory, such as the notions of fullness, faithfulness, 
limit, colimit, adjunction, Kan extensions (though I have not yet seen this in the literature), Yoneda embeddings, etc.

Example 2.2.8. There are tons of examples. I'll just list a few.

- Any set is a Segal $n$-category in the obvious way. This is not an interesting example.

- Carlos Simpson demonstrated that the category Se - $(n-1)$ - Cat can be viewed naturally as a Segal $n$-category.

- Any S-category can in particular be viewed as a Segal 1-category.

2.2.9. Observe that Segal 0 -categories are the same as $\infty$-pregroupoids. Preference for the latter terminology is justified and generalized by means of the existence of the free closed model structures introduced below.

\section{3 (Closed model structures for $(\infty, n)$-precategories)}

I briefly describe two closed model structures on the category of Segal $n-$ precategories.

Scholium 2.3.1. For every $n \geqslant 0$, there is a cofibrantly generated, internal closed model structure on $\mathbf{S e}-n-\mathbf{P C}$. These model structures possess the following properties.

- The model structure on $\mathbf{S e}-0-\mathbf{P C}$ is the model structure on $\infty$ pregroupoids.

- For any $n$, the cofibrations of this model structure are precisely the monomorphisms.

- Any fibrant object is a Segal n-category, but not conversely.

- A morphism $A \rightarrow B$ of Segal $n$-categories is a weak equivalence iff it is a Dwyer-Kan equivalence.

This closed model structure will be called the Hirschowitz-Simpson closed model structure.

About the Proof. This is proved using a complicated induction and the small object argument. A similar theorem for the Tamsamani-Simpson theory of $n$-categories appeared earlier.

2.3.2. In fact, not all objectwise fibrant Segal $n$-categories-Segal $n$ categories $A$ such that $A_{p}$ is a fibrant Segal $(n-1)$-category for every $p \in \Delta^{\mathrm{op}}$ — are fibrant. There are in some sense very few fibrant objects in the Hirschowitz-Simpson closed model structure, and they are very difficult to characterize. This motivates the following result of mine. 
Scholium 2.3.3. For every $n \geqslant 0$, there is a proper, combinatorial closed model structure on $\mathbf{S e}-n-\mathbf{P C}$. These model structures possess the following properties.

- The model structure on $\mathbf{S e - 0 - P C}$ is the Quillen closed model structure on $\infty$-pregroupoids.

- For any $n \geqslant 0$, the identity functor is a left Quillen equivalence from this model structure to the Hirschowitz-Simpson closed model structure.

- The cofibrations are certain "free" cofibrations.

- For any $n \geqslant 0$, the fibrant objects in this model structure are precisely those Segal $n$-categories $A$ such that for every $p \in \Delta^{\mathrm{op}}, A_{p}$ is fibrant in the free closed model structure on $\mathbf{S e}-(n-1)-\mathbf{P C}$.

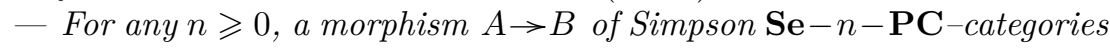
is a weak equivalence in this model structure iff it is a Dwyer-Kan equivalence.

About the Proof. A version of this theorem was proved for $n=1$ by J. Bergner in her thesis. The general case appears in my thesis. The proof proceeds by induction on $n$. For $n=0$, there is nothing to say. To construct the desired closed model structure on $\mathbf{S e}-n-\mathbf{P C}$, one defines a kind of projective closed model structure, takes a certain left Bousfield localization to ensure that the fibrant objects are exactly the objectwise fibrant Segal $n$-categories, and then takes a right Bousfield localiztion to ensure that the weak equivalences between fibrant objects are exactly the Dwyer-Kan equivalences.

Definition 2.3.4. A Segal $n$-precategory is called a $\operatorname{Simpson}(\infty, n)$ category if it is a fibrant object of the free closed model structure. In particular, Simpson $(\infty, 0)$-categories are exactly $\infty$-groupoids.

2.3.5. It is my view that Simpson $(\infty, n)$-categories are a better model for $(\infty, n)$-categories. By induction, they are Segal $n$-categories $A$ such that the simplicial set $A\left(m_{1}, \ldots, m_{n}\right)$ is fibrant for any $\left(m_{1}, \ldots, m_{n}\right) \in \Theta^{n}$.

It should be noted that the ease with which one can characterize fibrant objects in the free closed model structure has come at a price : it is not an internal closed model structure; there is no homotopically correct internal Mor functor in the free closed model structure.

2.3.6. The diagonal functor $\mathbf{S e}-n-\mathbf{P C} \rightarrow s$ Set can be viewed as the assignment that formally inverts all $i$-morphisms for $i \leqslant n$.

Lemma 2.3.7. The diagonal functor $\mathbf{S e}-n-\mathbf{P C} \rightarrow s$ Set is a left Quillen functor for both the Hirschowitz-Simpson and the free closed model structures. Its right adjoint is denoted $\Re$. 
2.4 (Descent for $(\infty, n)$-stacks). In this section, I explain descent for presheaves of $(\infty, n)$-categories, using the free closed model structure. This follows closely the discussion of stacks in $\infty$-groupoids. Again let $(T, \tau)$ be a site with enough points. Let $(\infty, n)-\mathbf{P S}(T)$ be the category of presheaves of Segal $n$-precategories. Recall that since the free closed model structure is combinatorial, $(\infty, n)-\mathbf{P S}(T)$ has a projective closed model structure.

Theorem 2.4.1. There is a left proper, combinatorial closed model structure on $(\infty, n)-\mathbf{P S}(T)$ such that the following conditions are satisfied.

- The cofibrations are precisely the projective cofibrations.

- The fibrant objects are exactly those presheaves of Simpson $(\infty, n)$ categories $F$ such that for any hypercovering $U \rightarrow X$ of any object $X$ of $T$, the induced morphism

$$
F X \rightarrow \operatorname{holim}\left[\prod_{\alpha_{0}} F U_{0}^{\alpha_{0}} \underset{\rightarrow}{\rightarrow} \prod_{\alpha_{1}} F U_{1}^{\alpha_{1}} \underset{\rightarrow}{\rightarrow} \ldots\right]
$$

is an equivalence of simplicial sets, where the $U_{n}^{\alpha_{n}}$ are the representing objects of the functors of which $U_{n}$ is the coproduct.

- The weak equivalences are exactly those morphisms $F \rightarrow G$ such that for any point $x$ of $T$, the induced morphism $F_{x} \rightarrow G_{x}$ is a Dwyer-Kan equivalence of Simpson $(\infty, n)$-categories.

This closed model structure is called the local projective closed model structure.

Proof. By using the functor $\mathbf{R}$, any hypercovering can be seen as a morphism of Segal $n$-precategories. Now, as before, one verifies that the left Bousfield localization of the projective closed model structure with respect to all hypercoverings exists and satisfies the listed properties.

Definition 2.4.2. An $(\infty, n)$-stack is a fibrant object of the local projective closed model structure on $(\infty, n)-\mathbf{P S}(T)$.

2.4.3. Finally, one can put forward the following question : is the assignment $\operatorname{Spec} R \mapsto L \mathbf{C p l x}(R)$ an $(\infty, 1)$-stack for any familiar topologies on the category of affine schemes? The answer is yes, but it turns out not to be so easy to verify this. Indeed, in the first version of Descente pour les $n$ Champs in 1998, Hirschowitz and Simpson gave a faulty proof of this. This result had to be partially retracted, and the authors were able to verify descent (for any subcanonical topology) only for the presheaf of positive complexes Spec $R \mapsto L \mathbf{C p l x}^{+}(R)$.

In my forthcoming note Redintegration of a Theorem of Hirschowitz and Simpson, I demonstrate that, as a consequence of a generalized tilting theorem of Schwede and Shipley, for any ring $R$, the closed model category $H R-$ Mod 
of modules over the Eilenberg-Mac Lane $E_{\infty}$ ring spectrum $H R$ is Quillen equivalent to the closed model category $\mathbf{C p l x}(R)$ of unbounded complexes, and, moreover, this Quillen equivalence gives rise to a functorial equivalence of Dwyer-Kan simplicial localizations $L(H R-\operatorname{Mod}) \rightarrow L \mathrm{Cplx}(R)$. In lieu of Spec $R \mapsto L \mathbf{C p l x}(R)$, one can therefore study the presheaf of Simpson $(\infty, 1)-$ categories $A \mapsto L(A-$ Mod $)$ on the opposite model category $\mathscr{A}_{H \mathbf{Z}}$ of the model category of $H \mathbf{Z}$-algebras, and pull back this presheaf along the EilenbergMac Lane functor $H$. Using Toën and Vezzosi's theory of homotopical algebraic geometry, I observe that for any subcanonical topology $\tau$ on the category of affine schemes, there is a topology on Ho $\mathscr{A}_{H \mathbf{Z}}$ such that the Eilenberg-Mac Lane functor $H$ is a continuous morphism of model sites, and the 1-prestack $A \mapsto L(A-$ Mod $)$ is a 1 -stack. A corollary is that the Hirschowitz-Simpson descent result for positive complexes can be extended to unbounded complexes, as it was originally envisioned.

Theorem 2.4.4. The 1-prestack $\operatorname{Spec} R \mapsto L \mathbf{C p l x}(R)$ is a 1 -stack for the category of affine schemes equipped with any subcanonical topology. 

Mathematisches Institut, Seminars, (Y. TschinkeL, ed.), p. 197-202

Universität Göttingen, 2004-05

\title{
SELF-DUAL NORMAL BASES AND TRACE FORMS OF GALOIS ALGEBRAS
}

\section{E. Lequeu}

Mathematisches Institut, Georg-August-Universität Göttingen, Bunsenstrasse 35, 37073 Göttingen, Germany • E-mail : lequeu@uni-math.gwdg.de

\begin{abstract}
In this note, we present a survey on the problem of the existence of a self-dual normal basis in a finite Galois extension and say how this problem is related to the study of trace forms of Galois algebras.
\end{abstract}

\section{Self-dual normal bases}

Let $K$ be a commutative field and $L$ be a finite Galois extension of $K$. We denote by $\Gamma$ the Galois group of $L / K$. A normal basis of $L / K$ is a $K$-basis of $L$ of the form $\left(\gamma\left(x_{0}\right)\right)_{\gamma \in \Gamma}$, for some $x_{0}$ in $L^{\times}$. It is well known that every finite Galois extension has a normal basis (cf. [Bou59] and [Bou52]). The trace form of $L / K$ is the non-degenerate symmetric bilinear form $b_{L}: L \times L \mapsto K$ defined by

for all $x, y \in L$.

$$
b_{L}(x, y)=\operatorname{tr}_{L / K}(x y)
$$

A normal basis $\left(\gamma\left(x_{0}\right)\right)_{\gamma \in \Gamma}$ of $L / K$ is said to be self-dual if

for all $\gamma, \gamma^{\prime} \in \Gamma$.

$$
b_{L}\left(\gamma\left(x_{0}\right), \gamma^{\prime}\left(x_{0}\right)\right)=\delta_{\gamma, \gamma^{\prime}}
$$

As we will see ( $c f$. Theorem 1.2), not every Galois extension has a self-dual normal basis. So, one can ask the following

$\overline{\text { January 20, 2005. }}$. 
Question 1.1. Which finite Galois extensions have a self-dual normal basis?

No complete answer to this question is known. Let us recall the main results on this topic. E. Bayer-Fluckiger and H. W. Lenstra, Jr. gave a complete criterion for the existence of a self-dual normal basis in an abelian extension (cf. [BFL90]) :

Theorem 1.2. Let $L / K$ be a finite abelian extension with Galois group $\Gamma$.

a) Assume that $\operatorname{char}(K) \neq 2$. Then $L / K$ has a self-dual normal basis if and only if $[L: K]$ is odd.

b) Assume that $\operatorname{char}(K)=2$. Then $L / K$ has a self-dual normal basis if and only if the exponent of $\Gamma$ is not divisible by 4.

They proved also that every Galois extension of odd degree has a self-dual normal basis ( $c f .[$ BF89] and [BFL90]) :

Theorem 1.3. Let $L / K$ be a finite Galois extension. If $L / K$ is of odd degree, then $L / K$ has a self-dual normal basis.

E. Bayer-Fluckiger and J.-P. Serre provided still partial results for the self-dual normal basis problem for Galois extensions of characteristic not 2 and of even degree (cf. [BFS94]).

\section{A reformulation of the problem}

We will see that the self-dual normal basis problem for a Galois extension with Galois group $\Gamma$ can be reformulated in terms of $\Gamma$-symmetric spaces.

Let $G$ be a finite group and $K$ a commutative field. $A$-symmetric space over $K$ is a pair $(V, q)$, where $V$ is a finite dimensional $K$-vector space with an operation of $G$ by automorphisms of $V$ and $b: V \times V \rightarrow K$ is a non-degenerate symmetric bilinear form such that

for all $u, v \in V$ and $g \in G$.

$$
b(g \cdot u, g \cdot v)=b(u, v)
$$

Examples 2.1.

a) Let $L / K$ be a finite Galois extension with Galois group $\Gamma$. Then the pair $\left(L, b_{L}\right)$ is a $\Gamma$-symmetric space over $K$ for the natural operation of $\Gamma$ on L.

b) Let $K[G]$ be the group algebra of $G$ over $K$ with the natural operation of $G$. We denote by $b_{0}: K[G] \times K[G] \rightarrow K$ the symmetric bilinear form defined by

$$
b_{0}(g, h)=\delta_{g, h}
$$


for all $g, h \in G$.

Then the pair $\left(K[G], b_{0}\right)$ is a $G$-symmetric space over $K$ called the unit $G$ symmetric space.

Let $(V, b)$ and $\left(V^{\prime}, b^{\prime}\right)$ two $G$-symmetric spaces over $K$. One says that $(V, b)$ and $\left(V^{\prime}, b^{\prime}\right)$ are isomorphic if there exists an isomorphism of $K$-vector spaces $f: V \rightarrow V^{\prime}$ such that

for all $v \in V$ and $g \in G$;

$$
f(g \cdot v)=g \cdot f(v)
$$

for all $u, v \in V$.

$$
b^{\prime}(f(u), f(v))=b(u, v)
$$

If so, one writes $(V, b) \simeq_{G}\left(V^{\prime}, b^{\prime}\right)$.

The following fact ( $c f$. [BFL90]) leads to a reformulation of the self-dual normal basis problem :

Proposition 2.2. Let $L / K$ be a finite Galois extension with Galois group $\Gamma$. The following conditions are equivalent :

a) $L / K$ has a self-dual normal basis.

b) $\left(L, b_{L}\right)$ and $\left(K[\Gamma], b_{0}\right)$ are isomorphic as $\Gamma$-symmetric spaces.

\section{Some notations}

From now, $G$ is a finite group and $K$ is a commutative field of char $(K) \neq 2$. Then symmetric bilinear forms over $K$ correspond bijectively to quadratic forms over $K$. We will consider quadratic forms instead of symmetric bilinear forms and $G$-quadratic spaces instead of $G$-symmetric spaces.

If $L$ is a finite Galois extension of $K$, we denote by $q_{L}: L \rightarrow K$ the quadratic form associated with the symmetric bilinear form $b_{L}: L \times L \rightarrow K$. We denote by $q_{0}: K[G] \rightarrow K$ the quadratic form associated with the symmetric bilinear form $b_{0}: K[G] \times K[G] \rightarrow K$.

Let $\operatorname{Quadr}(G, K)$ be the category of $G$-quadratic spaces over $K$. We will need the following functors, which are defined as usual ( $c f$. [Leq03]):

the scalar extension functor

$$
\text { Quadr }(G, K) \rightarrow \operatorname{Quadr}(G, E),(V, q) \mapsto(V, q)_{E},
$$

for any field extension $E / K$;

the restriction functor

$$
\text { Quadr }(G, K) \rightarrow \operatorname{Quadr}(S, K),(V, q) \mapsto \operatorname{Res}_{S}^{G}(V, q),
$$

for any subgroup $S$ of $G$;

the induction functor 
Quadr $(S, K) \rightarrow \operatorname{Quadr}(G, K),(V, q) \mapsto \operatorname{Ind}_{S}^{G}(V, q)$, for any subgroup $S$ of $G$.

\section{Odd degree extensions}

One has the following result $(c f .[\mathbf{C P 8 4}])$ :

Lemma 4.1. Let $L$ be a finite Galois extension of $K$ with Galois group $\Gamma$. Then the $\Gamma$-quadratic spaces $\left(L, q_{L}\right)_{L}$ and $\left(K[\Gamma], q_{0}\right)_{L}$ over $L$ are isomorphic.

Moreover, in [BFL90], E. Bayer-Fluckiger and H. W. Lenstra, Jr. prove the

Theorem 4.2. Let $(V, q)$ and $\left(V^{\prime}, q^{\prime}\right)$ be two G-quadratic spaces over $K$. Let $E$ be an extension of $K$ of odd degree. Then $(V, q) \simeq_{G}\left(V^{\prime}, q^{\prime}\right)$ if and only if $(V, q)_{E} \simeq_{G}\left(V^{\prime}, q^{\prime}\right)_{E}$.

It follows from Lemma 4.1, Theorem 4.2 and Proposition 2.2 that every Galois extension $L$ of $K$ of odd degree has a self-dual normal basis ( $c f$. th. $1.3)$.

\section{Galois algebras}

In the previous section, we have seen that it can be useful to pass to finite extensions of the base field. In order to do this, one has to consider not only Galois extensions but Galois algebras.

$A$ Galois algebra over $K$ is an étale $K$-algebra $L$ with an operation of $G$ by automorphisms of $L$ such that $G$ acts simply and transitively on the set $\operatorname{Hom}_{K-a l g}\left(L, K_{\mathrm{S}}\right)$, where $K_{\mathrm{S}}$ is a separable closure of $K$.

Two $G$-Galois algebras $L$ and $L^{\prime}$ over $K$ are said to be isomorphic if there exists an isomorphism of $K$-algebras $f: L \rightarrow L^{\prime}$ such that

for all $x \in L$ and $g \in G$.

$$
f(g \cdot x)=g \cdot f(x)
$$

Let $L$ be a $G$-Galois algebra over $K$. We still denote by $q_{L}: L \rightarrow K$ the (quadratic) trace form of $L$. Then $\left(L, q_{L}\right)$ is a $G$-quadratic space over $K$.

Example 5.1. The split G-Galois algebra over $K$ is $L_{0}=K \times \ldots \times K$, where $G$ acts by permuting the factors simply and transitively. One has $\left(L_{0}, q_{L_{0}}\right) \cong_{G}$ $\left(K[G], q_{0}\right)$. 
Let $\operatorname{Gal}(G, K)$ be the category of $G$-Galois algebras over $K$. One defines as usual the two following functors $(c f$. [Leq03]):

the scalar extension functor

for any field extension $E / K$;

$$
\operatorname{Gal}(G, K) \rightarrow \operatorname{Gal}(G, E), L \mapsto L_{E},
$$

the induction functor

for any subgroup $S$ of $G$.

$$
\operatorname{Gal}(S, K) \rightarrow \operatorname{Gal}(G, K), M \mapsto \operatorname{Ind}_{S}^{G} M,
$$

\section{The induction problem}

Of course, the self-dual normal basis problem can be raised in the context of Galois algebras. The reformulation of this problem leads to study the classification of the $G$-quadratic spaces $\left(L, q_{L}\right)$, for $L$ a $G$-Galois algebra ( $c f$. Proposition 2.2 and Example 5.1). In order to do this, E. Bayer-Fluckiger and J.-P. Serre developed in [BFS94] the following method.

Proposition 6.1. Let $L$ be a $G$-Galois algebra over $K$. Let $S$ be a 2-Sylow subgroup of $G$. Then there exist $E$ an extension of $K$ of odd degree and $M a$ $S$-Galois algebra over $E$ such that $L_{E}$ and $\operatorname{Ind}_{S}^{G} M$ are isomorphic as $G$-Galois algebras over $E$.

Let $L$ and $L^{\prime}$ be two $G$-Galois algebras over $K$. Let $S$ be a 2-Sylow subgroup of $G$. It follows from Proposition 6.1 that there exist $E$ an extension of $K$ of odd degree and $M$ and $M^{\prime}$ two $S$-Galois algebras over $E$ such that the $G$ Galois algebras $L_{E}$ and $\operatorname{Ind}_{S}^{G} M$ are isomorphic and the $G$-Galois algebras $L_{E}^{\prime}$ and $\operatorname{Ind}_{S}^{G} M^{\prime}$ are isomorphic. Then, from th. 4.2, one has:

$$
\begin{aligned}
\left(L, q_{L}\right) \simeq_{G}\left(L^{\prime}, q_{L^{\prime}}\right) & \Leftrightarrow\left(L, q_{L}\right)_{E} \simeq_{G}\left(L^{\prime}, q_{L^{\prime}}\right)_{E} \\
& \Leftrightarrow \operatorname{Ind}_{S}^{G}\left(M, q_{M}\right) \simeq_{G} \operatorname{Ind}_{S}^{G}\left(M^{\prime}, q_{M^{\prime}}\right) .
\end{aligned}
$$

This leads to raise the induction problem:

Problem 6.2. Let $S$ be a 2-Sylow subgroup of $G$. Let $\left(V_{1}, q_{1}\right)$ and $\left(V_{2}, q_{2}\right)$ be two $S$-quadratic spaces over $K$. Could one give necessary and sufficient conditions for the $G$-quadratic spaces $\operatorname{Ind}_{S}^{G}\left(V_{1}, q_{1}\right)$ and $\operatorname{Ind}_{S}^{G}\left(V_{2}, q_{2}\right)$ to be isomorphic?

E. Bayer-Fluckiger and J.-P. Serre gave such conditions in the case where $S$ is elementary abelian ( $c f$. [BFS94]). Their result can be generalized to the case where $S$ is abelian $(c f$. [Leq03]) and be formulated as follows: 
Theorem 6.3. Let $S$ be a 2-Sylow subgroup of $G$. Suppose that $S$ is abelian. Let $N$ be the normalizer of $S$ in $G$. Let $\left(V_{1}, q_{1}\right)$ and $\left(V_{2}, q_{2}\right)$ be two $S$-quadratic spaces over $K$. Then the following conditions are equivalent:

a) The $G$-quadratic spaces $\operatorname{Ind}_{S}^{G}\left(V_{1}, q_{1}\right)$ and $\operatorname{Ind}_{S}^{G}\left(V_{2}, q_{2}\right)$ are isomorphic.

b) The $S$-quadratic spaces $\operatorname{Res}_{S}^{G} \operatorname{Ind}_{S}^{G}\left(V_{1}, q_{1}\right)$ and $\operatorname{Res}_{S}^{G} \operatorname{Ind}_{S}^{G}\left(V_{2}, q_{2}\right)$ are isomorphic.

c) The $N$-quadratic spaces $\operatorname{Ind}_{S}^{N}\left(V_{1}, q_{1}\right)$ and $\operatorname{Ind}_{S}^{N}\left(V_{2}, q_{2}\right)$ are isomorphic.

Under certain additional hypotheses, these results let to state cohomological criteria for the isomorphy of the $G$-quadratic spaces $\left(L, q_{L}\right)$ and $\left(L^{\prime}, q_{L^{\prime}}\right)$, for $L$ and $L^{\prime}$ two $G$-Galois algebras, and, consequently, for the existence of a self-dual normal basis in a $G$-Galois algebra ( $c f .[$ BFS94] and [Leq03]).

\section{References}

[BF89] E. BAyer-Fluckiger - Self-dual normal bases, Nederl. Akad. Wetensch. Indag. Math. 51 (1989), no. 4, p. 379-383.

[BFL90] E. BAyer-Fluckiger \& H. W. Lenstra, JR. - Forms in odd degree extensions and self-dual normal bases, Amer. J. Math. 112 (1990), no. 3, p. 359-373.

[BFS94] E. BAyer-Fluckiger \& J.-P. SerRe - Torsions quadratiques et bases normales autoduales, Amer. J. Math. 116 (1994), no. 1, p. 1-64.

[Bou52] N. Bourbaki - Éléments de mathématique. XIV. Première partie: Les structures fondamentales de l'analyse. Livre II: Algèbre. Chapitre VI: Groupes et corps ordonnés. Chapitre VII: Modules sur les anneaux principaux, Actualités Sci. Ind., no. 1179, Hermann et Cie, Paris, 1952.

[Bou59]__ Éléments de mathématique. I: Les structures fondamentales de l'analyse. Fascicule XI. Livre II: Algèbre. Chapitre 4: Polynomes et fractions rationnelles. Chapitre 5: Corps commutatifs, Deuxième édition. Actualités Scientifiques et Industrielles, No. 1102, Hermann, Paris, 1959.

[CP84] P. E. Conner \& R. PERLIS - A survey of trace forms of algebraic number fields, Series in Pure Mathematics, vol. 2, World Scientific Publishing Co., Singapore, 1984.

[Leq03] E. LeqUeU - $g$-formes $\varepsilon$-Hermitiennes et Induction, Formes Trace de $g$ Algèbres Galoisiennes, 2003, thèse. 
Mathematisches Institut, Seminars, (Y. TschinkeL, ed.), p. 203-209

Universität Göttingen, 2004-05

\title{
THE DIOPHANTINE EQUATION $x^{4}+2 y^{4}=z^{4}+4 w^{4}$
}

\section{A.-S. Elsenhans}

\section{J. Jahnel}

Mathematisches Institut, Bunsenstr. 3-5, 37073 Göttingen, Germany

E-mail : jahnel@uni-math.gwdg.de

\begin{abstract}
We show that, within the hypercube $|x|,|y|,|z|,|w| \leqslant 2.5 \cdot 10^{6}$, the Diophantine equation $x^{4}+2 y^{4}=z^{4}+4 w^{4}$ admits essentially one and only one non-trivial solution, namely

$$
\text { ( } \pm 1484801, \pm 1203120, \pm 1169407, \pm 1157520) \text {. }
$$
\end{abstract}

The investigation is based on a systematic search by computer.

\section{Introduction}

1.1. An algebraic curve $C$ of genus $g>1$ has at most a finite number of $\mathbb{Q}$-rational points. On the other hand, for genus one curves, $\# C(\mathbb{Q})$ may be zero, finite nonzero, or infinite. For genus zero curves, one automatically has $\# C(\mathbb{Q})=\infty$ as soon as $C(\mathbb{Q}) \neq \emptyset$.

\section{January 2005.}

The first author was partially supported by a Doctoral Fellowship of the Deutsche Forschungsgemeinschaft (DFG). The computer part of this work was executed on the Linux PCs of the Gauss Laboratory for Scientific Computing at the Göttingen Mathematical Institute. Both authors are grateful to Prof. Y. Tschinkel for the permission to use these machines as well as to the system administrators for their support. 
1.2. In higher dimensions, there is a conjecture, due to S. Lang, stating that if $X$ is a variety of general type over a number field then all but finitely many of its rational points are contained in the union of closed subvarieties which are not of general type. On the other hand, abelian varieties (as well as, e.g., elliptic and bielliptic surfaces) behave like genus one curves, i.e., $\# X(\mathbb{Q})$ may be zero, finite nonzero, or infinite. Finally, rational and ruled varieties behave in many respects like genus zero curves.

This list does not yet exhaust the classification of algebraic surfaces, to say nothing of dimension three or higher. In particular, the following problem is still open.

Problem. 1.1. Does there exist a K3 surface over $\mathbb{Q}$ which has a finite nonzero number of $\mathbb{Q}$-rational points, i.e., such that $0<\# X(\mathbb{Q})<\infty$ ?

Remark. 1.2. This question was posed by Sir P. Swinnerton-Dyer as Problem/Question 6.a) in the problem session of the workshop [PT04]. We are not able to give an answer to it.

Problem. 1.3. Find a third point on the projective surface $S \subset \mathbf{P}^{3}$ defined by

$$
x^{4}+2 y^{4}=z^{4}+4 w^{4} .
$$

Remarks. 1.4. i) The equation $x^{4}+2 y^{4}=z^{4}+4 w^{4}$ defines a K3 surface $S$ in $\mathbf{P}^{3}$. As trivial solutions of the equation, we consider those corresponding to the $\mathbb{Q}$-rational points $(1: 0: 1: 0)$ and $(1: 0:(-1): 0)$.

ii) Problem 1.3 is also due to Sir P. Swinnerton-Dyer [PT04, Question 6.c)]. It was raised in particular during his talk [SD, very end of the article] at the Göttingen Mathematisches Institut on June 2nd, 2004.

Our main result is the following theorem containing an answer to Problem 1.3.

Theorem. 1.5. The diagonal quartic surface in $\mathbf{P}^{3}$ defined by

$$
x^{4}+2 y^{4}=z^{4}+4 w^{4}
$$

admits precisely ten $\mathbb{Q}$-rational points which allow integral coordinates within the hypercube $|x|,|y|,|z|,|w|<2.5 \cdot 10^{6}$.

These are $( \pm 1: 0: \pm 1: 0)$ and

$$
( \pm 1484801: \pm 1203120: \pm 1169407: \pm 1157520) .
$$




\section{Congruences}

2.1. It seems natural to first try to understand the congruences

$$
x^{4}+2 y^{4} \equiv z^{4}+4 w^{4} \quad(\bmod p)
$$

modulo a prime number $p$. For $p=2$ and 5 , one finds that all primitive solutions in $\mathbb{Z}$ satisfy

a) $x$ and $z$ are odd,

b) $y$ and $w$ are even,

c) $y$ is divisible by 5 .

For other primes, the Weil conjectures, proven by P. Deligne [Del74], imply that the number of solutions to the congruence $(+)$ is $1+(p-1)\left(p^{2}+p+1+E\right)$, where $E$ is an error-term which may be estimated by $|E| \leqslant 21 p$.

Indeed, let $X$ be the projective variety over $\mathbb{Q}$ defined by $(*)$. It has good reduction at every prime $p \neq 2$. Therefore, [Del74, Théorème (8.1)] may be applied to the reduction $X_{p}$. This yields $\# X_{p}\left(\mathbb{F}_{p}\right)=p^{2}+p+1+E$ and $|E| \leqslant 21 p$. We note that $\operatorname{dim} H^{2}(\mathscr{X}, \mathbb{R})=22$ for every complex surface $\mathscr{X}$ of type $K 3$ [Ber01, p. 98].

2.2. Another question of interest is to count the numbers of solutions to the congruences $x^{4}+2 y^{4} \equiv c(\bmod p)$ and $z^{4}+4 w^{4} \equiv c(\bmod p)$ for a certain $c \in \mathbb{Z}$.

This means to count the $\mathbb{F}_{p}$-rational points on the affine plane curves $C_{c}^{l}$ and $C_{c}^{r}$ defined over $\mathbb{F}_{p}$ by $x^{4}+2 y^{4}=\bar{c}$ and $z^{4}+4 w^{4}=\bar{c}$, respectively. If $p \nmid c$ and $p \nmid 2$ then these are smooth curves of genus three.

By the work of André Weil [Wei48, Corollaire 3 du Théorème 13], the numbers of $\mathbb{F}_{p}$-rational points on their projectivizations are given by

$$
\# \bar{C}_{c}^{l}\left(\mathbb{F}_{p}\right)=p+1+E_{l} \quad \text { and } \quad \# \bar{C}_{c}^{r}\left(\mathbb{F}_{p}\right)=p+1+E_{r},
$$

where the error-terms can be bounded by $\left|E_{l}\right|,\left|E_{r}\right| \leqslant 6 \sqrt{p}$. There may be up to four $\mathbb{F}_{p}$-rational points on the infinite line.

For our purposes, it suffices to notice that both congruences have approximately $p$ solutions.

The case $p \mid c, p \neq 2$ is slightly different since it corresponds to the case of a reducible curve. The congruence $x^{4}+k y^{4} \equiv 0(\bmod p)$ admits only the trivial solution if $(-k)$ is not a biquadratic residue modulo $p$. Otherwise, it has exactly $1+(p-1) \operatorname{gcd}(p-1,4)$ solutions.

Finally, if $p=2$ then, $\# C_{0}^{l}\left(\mathbb{F}_{p}\right)=\# C_{0}^{r}\left(\mathbb{F}_{p}\right)=p$ and $\# C_{1}^{l}\left(\mathbb{F}_{p}\right)=\# C_{1}^{r}\left(\mathbb{F}_{p}\right)=2 p$. 
Remark. 2.1. The number of solutions to the congruence $(+)$ is

$$
\sum_{c \in \mathbb{F}_{p}} \# C_{c}^{l}\left(\mathbb{F}_{p}\right) \cdot \# C_{c}^{r}\left(\mathbb{F}_{p}\right) .
$$

Hence, the formulas just mentioned yield an elementary estimate for that count. This shows again that the dominating term is $p^{3}$. The estimate for the error is, however, less sharp than the one obtained via the more sophisticated methods in 2.1.

\section{Naive methods}

3.1. The most naive method to search for solutions to $(*)$ is probably the following: Start with the set

$$
\{(x, y, z, w) \in \mathbb{Z} \mid 0 \leqslant x, y, z, w<N\}
$$

and test the equation for every quadruple.

Obviously this method requires about $N^{4}$ steps. It can be accelerated using the congruence conditions for primitive solutions noticed above.

3.2. A somewhat better method is to start with the set

$$
\left\{x^{4}+2 y^{4}-4 w^{4} \mid x, y, w \in \mathbb{Z}, 0 \leqslant x, y, w<N\right\}
$$

and search for fourth powers. This set has about $N^{3}$ Elements, and the algorithm takes about $N^{3}$ steps. Again, it can be sped up by the above congruence conditions for primitive solutions. We used this approach for a trial-run with $N=10^{4}$.

An interesting aspect of this algorithm is the optimization by further congruences. Suppose $x$ and $y$ are fixed, then about one half or tree quarter of the values for $w$ are no solutions for the congruence modulo a new prime. Following this way, one can find more congruences for $w$ and the size of the set may be reduced by a constant factor.

\section{Our final algorithm}

4.1. The basic idea. We need to compute the intersection of two sets

$$
\left\{x^{4}+2 y^{4} \mid x, y \in \mathbb{Z}, 0 \leqslant x, y<N\right\} \cap\left\{z^{4}+4 w^{4} \mid z, w \in \mathbb{Z}, 0 \leqslant z, w<N\right\} .
$$

Both have about $N^{2}$ elements.

It is a standard problem in Computer Science to find the intersection if both sets fit into memory. Using the congruence conditions above, one can reduce 
the size of the first set by a factor of 20 and the size of the second set by a factor of 4 .

4.2. Some details. The two sets described above are too big, at least for our computers and interesting values of $N$. Therefore, we introduced a prime number that we call the page prime $p_{p}$.

We define the sets

$$
L_{c}:=\left\{x^{4}+2 y^{4} \mid x, y \in \mathbb{Z}, 0 \leqslant x, y<N, x^{4}+2 y^{4} \equiv c\left(\bmod p_{p}\right)\right\}
$$

and

$$
R_{c}:=\left\{z^{4}+4 w^{4} \mid z, w \in \mathbb{Z}, 0 \leqslant z, w<N, z^{4}+4 w^{4} \equiv c\left(\bmod p_{p}\right)\right\} .
$$

Then, the intersection problem is divided into $p_{p}$ pieces and the sets $L_{c}$ and $R_{c}$ fit into the computer's memory if $p_{p}$ is big enough. We worked with $N=2.5 \cdot 10^{6}$ and chose $p_{p}=30011$.

For every value of $c$, the program computes $L_{c}$ and stores this set in a hash table. Then, it determines the elements of $R_{c}$ and looks them up in the hash table. The running-time of this algorithm is $O\left(N^{2}\right)$.

Remark. 4.2.1. An important further aspect of this approach is that the problem may be attacked in parallel on several machines. The calculations for one particular value of $c$ are independent of the analogous calculations for another one. Thus, it is possible, say, to let c run from 0 to $\left(p_{p}-1\right) / 2$ on one machine and, at the same time, from $\left(p_{p}+1\right) / 2$ to $\left(p_{p}-1\right)$ on another.

\subsection{Some more details.}

4.3.1. The page prime. For each value of $c$, it is necessary to find the solutions of the congruences $x^{4}+2 y^{4} \equiv c\left(\bmod p_{p}\right)$ and $z^{4}+4 w^{4} \equiv c\left(\bmod p_{p}\right)$ in an effective manner. We do this in a rather naive way by letting $y(w)$ run from 0 to $p-1$. For each value of $y(w)$, we compute $x^{4}\left(z^{4}\right)$. Then, we extract the fourth root modulo $p_{p}$.

Note that the page prime fulfills $p_{p} \equiv 3(\bmod 4)$. Hence, the fourth roots of unity modulo $p$ are just \pm 1 and, therefore, a fourth root modulo $p_{p}$, if it exists, is unique up to sign. This makes the algorithm easier to implement.

4.3.2. Actually, we do not do any modular powering operation or even computation of fourth roots in the lion's share of the running time. For more efficiency, all fourth powers and all fourth roots modulo $p_{p}$ are computed and stored in an array in an initialization step. Thus, the main speed limitation to find all solutions to a congruence modulo $p_{p}$ is, in fact, the time it takes to look up values stored in the machine's main memory. 
4.3.3. Hashing. We do not compute $L_{c}$ and $R_{c}$ directly, because this would require the use of multi-precision integers, all the time. Instead, we chose two other primes, the hash prime $p_{h}$ and the control prime $p_{c}$, which fit into the 32-bit registers of the computer. All computations are done modulo $p_{h}$ and $p_{c}$.

Precisely, for each pair $(x, y)$ considered, the expression $\left(\left(x^{4}+2 y^{4}\right) \bmod p_{h}\right)$ defines its position in the hash table. In other words, we hash pairs $(x, y)$ and $(x, y) \mapsto\left(\left(x^{4}+2 y^{4}\right) \bmod p_{h}\right)$ plays the role of the hash function.

For such a pair $(x, y)$, we write two entries into the hash table, namely $\left(\left(x^{4}+2 y^{4}\right) \bmod p_{c}\right)$ and $y$.

In the main computation, we worked with the values $p_{h}=25000009$ and $p_{c}=400000009$.

4.3.4. Note that, when working with a particular value of $c$, there are around $p_{p}$ pairs $\left(\left(x \bmod p_{p}\right),\left(y \bmod p_{p}\right)\right)$ which fulfill the required congruence

$$
x^{4}+2 y^{4} \equiv c \quad\left(\bmod p_{p}\right) .
$$

Therefore, the hash table will be filled with approximately

$$
p_{p} \cdot\left(\frac{N / 2}{p_{p}} \cdot \frac{N / 10}{p_{p}}\right)=\frac{N^{2}}{20 p_{p}}
$$

values. For our choices, $\frac{N^{2}}{20 p_{p}} \approx 10412849$ which means that the hash table will get approximately $41.7 \%$ filled.

Like for many other rules, there is an exception to this one. If $c=0$ then approximately $1+\left(p_{p}-1\right) \operatorname{gcd}\left(p_{p}-1,4\right)$ pairs $\left(\left(x \bmod p_{p}\right),\left(y \bmod p_{p}\right)\right)$ may satisfy the congruence

$$
x^{4}+2 y^{4} \equiv 0 \quad\left(\bmod p_{p}\right) .
$$

As $p_{p} \equiv 3(\bmod 4)$ this is not more than $2 p_{p}-1$ and the hash table will be filled not more than about $83.3 \%$.

4.3.5. To resolve collisions in the hash table, we use an open addressing method. We are not particularly afraid of clustering and choose linear probing.

4.3.6. Miscellanea. The program makes frequent use of fourth powers modulo $p_{h}$ and $p_{c}$. Again, we compute these data in the initialization part of our program and store them in arrays, once and for all.

4.3.7. Test versions of the program were written in Delphi. The final version was written in $\mathrm{C}$. It took about 130 hours of CPU time. 


\subsection{Post-processing.}

4.4.1. Instead of looking for solutions $x^{4}+2 y^{4}=z^{4}+4 w^{4}$, our algorithm looks for solutions to corresponding simultaneous congruences modulo $p_{p}$ and $p_{c}$ which, in addition, are such that $\left(\left(x^{4}+2 y^{4}\right) \bmod p_{h}\right)$ and $\left(\left(z^{4}+4 w^{4}\right) \bmod p_{h}\right)$ are "almost equal".

We found about 3800 solutions to this modified problem. These congruence solutions were checked by an exact computation using O. Forster's Pascalstyle multi-precision interpreter language ARIBAS.

4.4.2. Except for trivial solutions, equality occurs only once. This solution is as follows.

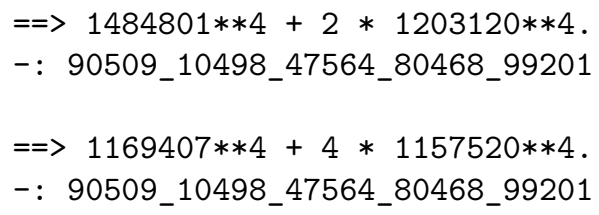

\section{References}

[Ber01] D. J. Bernstein - Enumerating solutions to $p(a)+q(b)=r(c)+s(d)$, Math. Comp. 70 (2001), no. 233, p. 389-394.

[Del74] P. Deligne - La conjecture de Weil. I, Inst. Hautes Études Sci. Publ. Math. (1974), no. 43, p. 273-307.

[PT04] B. Poonen \& Y. Tschinkel (eds.) - Arithmetic of higher-dimensional algebraic varieties, Progress in Mathematics, vol. 226, Boston, MA, Birkhäuser Boston Inc., 2004.

[SD] S. P. SwinnerTon-Dyer - Rational points on fibered surfaces, in: Tschinkel, Y. (ed.): Mathematisches Institut, Seminars 2004, Universitätsverlag, Göttingen 2004, p. 103-109.

[Wei48] A. WEIL - Sur les courbes algébriques et les variétés qui s'en déduisent, Actualités Sci. Ind., no. 1041 = Publ. Inst. Math. Univ. Strasbourg 7 (1945), Hermann et Cie., Paris, 1948. 
